\title{
WestVirginiaUniversity
}

THE RESEARCH REPOSITORY @ WVU

Graduate Theses, Dissertations, and Problem Reports

2001

\section{Optimum hybrid vehicle configurations for heavy duty applications}

Jonathan Burke Smith

West Virginia University

Follow this and additional works at: https://researchrepository.wvu.edu/etd

\section{Recommended Citation}

Smith, Jonathan Burke, "Optimum hybrid vehicle configurations for heavy duty applications" (2001). Graduate Theses, Dissertations, and Problem Reports. 1167.

https://researchrepository.wvu.edu/etd/1167

This Thesis is protected by copyright and/or related rights. It has been brought to you by the The Research Repository @ WVU with permission from the rights-holder(s). You are free to use this Thesis in any way that is permitted by the copyright and related rights legislation that applies to your use. For other uses you must obtain permission from the rights-holder(s) directly, unless additional rights are indicated by a Creative Commons license in the record and/ or on the work itself. This Thesis has been accepted for inclusion in WVU Graduate Theses, Dissertations, and Problem Reports collection by an authorized administrator of The Research Repository @ WVU. For more information, please contact researchrepository@mail.wvu.edu. 


\title{
Optimum Hybrid Vehicle Configurations for Heavy Duty Applications
}

\section{Jonathan Burke Smith}

Thesis submitted to

The College of Engineering and Mineral Resources

\author{
at \\ West Virginia University \\ in partial fulfillment of the requirements \\ for the degree of \\ Master of Science \\ in \\ Mechanical Engineering
}

Nigel N. Clark, Ph.D., Chair

Christopher M. Atkinson, Sc. D.

Thomas R. Long, Ph. D

Department of Mechanical and Aerospace Engineering

Morgantown, West Virginia 


\section{Abstract \\ Optimum Hybrid Vehicle Configurations for Heavy Duty Applications \\ Jonathan Burke Smith}

Increased concern about the fuel economy of and emissions from automobiles has led to interest in the use of hybrid electric powertrains and the introduction of several production vehicles in both heavy-duty and light-duty applications. Hybrid electric vehicles (HEVs) use a combination of electric motor(s) and another power source such as an internal combustion engine (ICE) or fuel cell. While these vehicles show great potential for use in a wide variety of driving situations, the optimization of components and control strategies is quite complex.

In this thesis, Class 2B, Class 6, and Class 8 vehicles are determined by averaging a variety of actual vehicles from each class and are simulated in Microsoft Excel over a variety of driving cycles to attempt to optimize their design and control. The drive cycles are modified to represent realistic expectations of the dynamic performance of vehicles from each class. Two types of hybrid powertrains are simulated. The series HEV is propelled solely by electric motors with energy coming from batteries and an alternator driven by an ICE. The parallel HEV is propelled by both electric motors and an ICE with charging-while-driving capabilities. The model is based on power requirements for each vehicle class and addresses concerns such as engine, battery, and driveline efficiencies. The control strategy forces the engine to run at a fixed percentage of the power required at the wheels plus or minus a battery state of charge correction factor.

Fuel economy increases of 100 to 150 percent were seen for Class 6 and 8 vehicles on transient cycles while 10 to 20 percent increases were seen on more constant speed cycles. The Yard cycle, a low average demand, highly transient cycle, was shown to be particularly suited to HEVs. 


\section{Table of Contents}

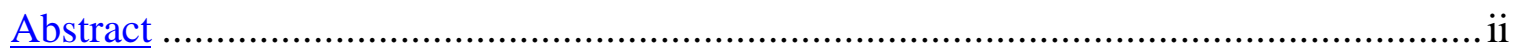

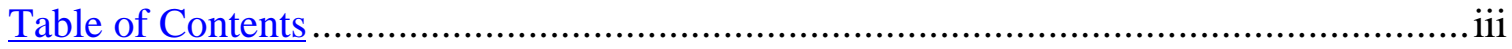

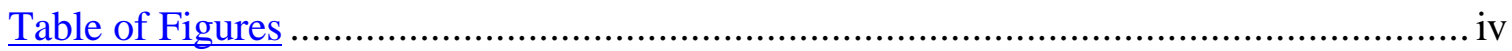

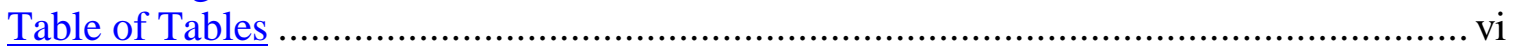

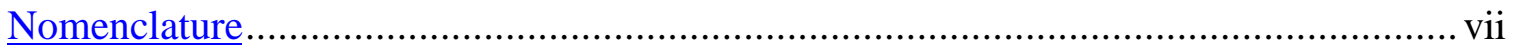

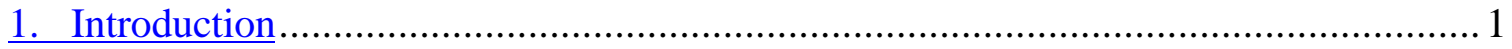

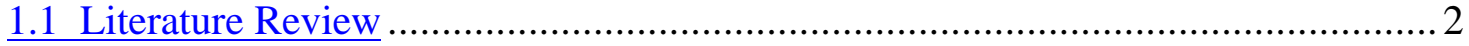

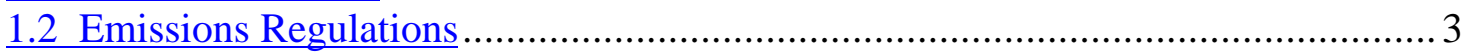

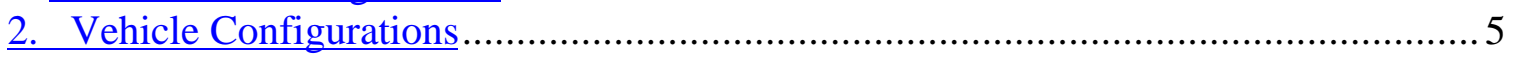

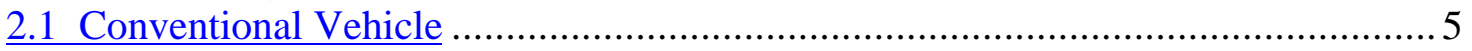

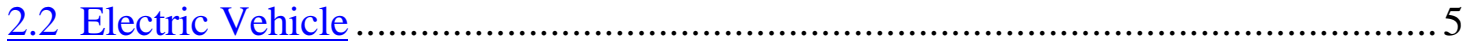

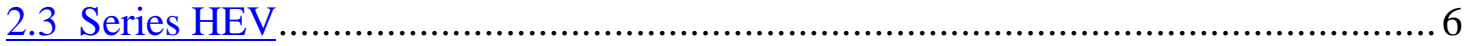

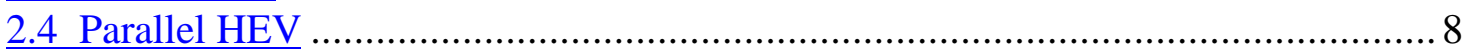

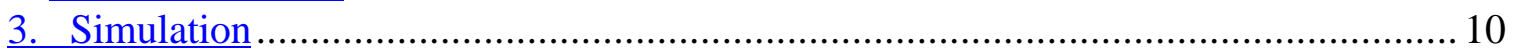

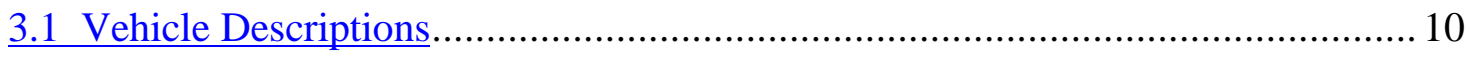

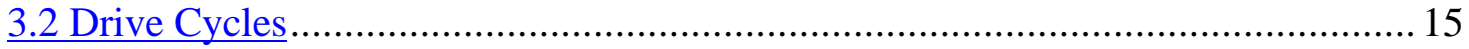

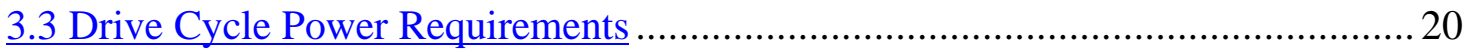

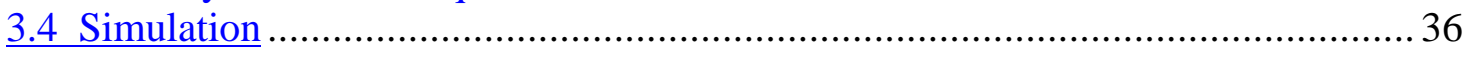

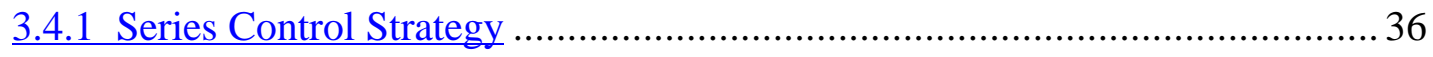

3.4.2 Parallel Control Strategy .............................................................................. 36

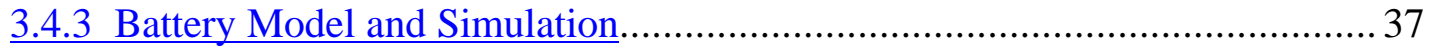

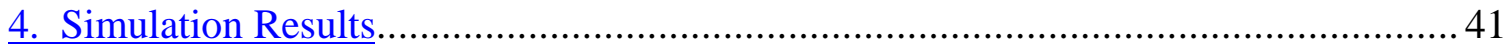

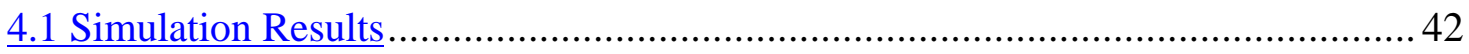

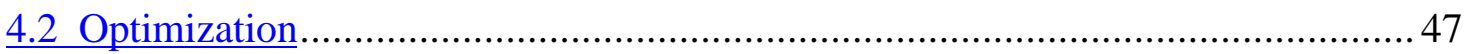

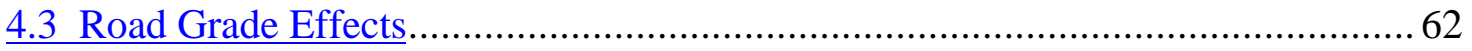

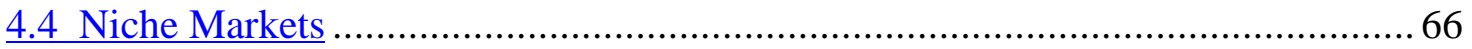

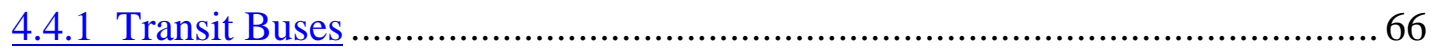

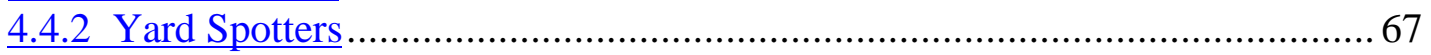

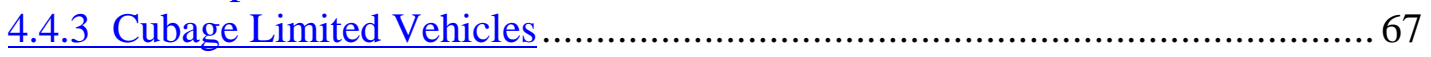

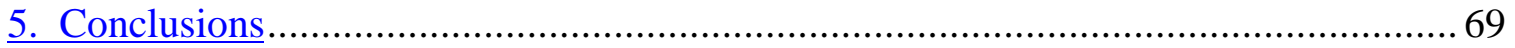

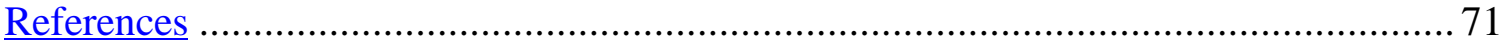




\section{Table of Figures}

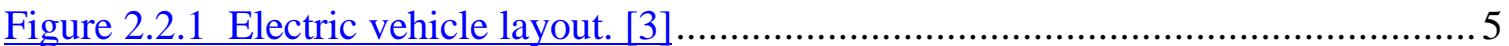

Figure 2.3.1 Series HEV layout. [3] .....................................................................

Figure 2.4.1 Parallel HEV layout with electric power delivered between ICE and

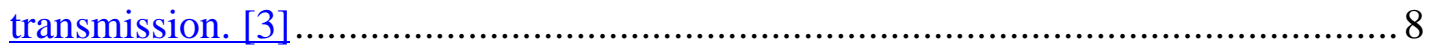

Figure 3.1.1 Class 2B vehicle power and weight. ………......................................... 11

Figure 3.1.2 Class 6 vehicle power and weight.......................................................... 12

Figure 3.1.3 Class 8 vehicle power and weight....................................................... 13

Figure 3.1.4 Typical Power / Weight ratios for various vehicle classes. ........................ 15

Figure 3.2.1 Speed - Time trace for the Freeway Cycle................................................ 16

Figure 3.2.2 Speed - Time trace for the CSHVC. .................................................... 16

Figure 3.2.3 Speed - Time trace for the Yard Cycle. .................................................. 17

Figure 3.2.4 Speed - Time trace for the Manhattan Cycle............................................. 18

Figure 3.2.5 Speed - Time trace for the Test D Cycle................................................. 19

Figure 3.2.6 Speed - Time trace for the Combined Cycle.............................................. 20

Figure 3.3.1 Power required for Class 2B vehicle over the Freeway Cycle. ................. 24

Figure 3.3.2 Power required for Class 6 vehicle over the Freeway Cycle..................... 25

Figure 3.3.3 Power required for Class 8 vehicle over the Freeway Cycle...................... 25

Figure 3.3.4 Power required for Class 2B vehicle over the CSHVC..............................2 26

Figure 3.3.5 Power required for Class 6 vehicle over the CSHVC. .............................. 27

Figure 3.3.6 Power required for Class 8 vehicle over the CSHVC. ……...................... 27

Figure 3.3.7 Power required for Class 2B vehicle over the Yard Cycle........................ 28

Figure 3.3.8 Power required for Class 6 vehicle over the Yard Cycle............................ 29

Figure 3.3.9 Power required for Class 8 vehicle over the Yard Cycle............................ 29

Figure 3.3.10 Power required for Class 2B vehicle over the Manhattan Cycle. ……..... 30

Figure 3.3.11 Power required for Class 6 vehicle over the Manhattan Cycle................. 31

Figure 3.3.12 Power required for Class 8 vehicle over the Manhattan Cycle................. 31

Figure 3.3.13 Power required for Class 2B vehicle over the Test D Cycle. ………........ 32

Figure 3.3.14 Power required for Class 6 vehicle over the Test D Cycle........................ 33

Figure 3.3.15 Power required for Class 8 vehicle over the Test D Cycle....................... 33

Figure 3.3.16 Power required for Class 2B vehicle over the Combined Cycle............... 34

Figure 3.3.17 Power required for Class 6 vehicle over the Combined Cycle. ................ 35

Figure 3.3.18 Power required for Class 8 vehicle over the Combined Cycle. ................ 35

Figure 3.4.1 Battery voltage vs. SoC for Hawker Genesis G13EP. [16] ........................ 38

Figure 3.4.2 Battery efficiency vs. current. [17]....................................................... 39

Figure 4.1.1 Class 6 Parallel HEV on Freeway Cycle without auxiliary load, $\mathrm{C}_{1}=0.2, \mathrm{C}_{2}$

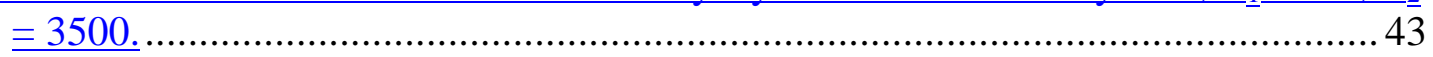

Figure 4.1.2 Expanded portion of Figure 4.1.1 from 1200 to 1400 seconds................... 44

Figure 4.1.3 Class 8 Series HEV on Manhattan Cycle with auxiliary load, $\mathrm{C}_{1}=0.2, \mathrm{C}_{2}=$

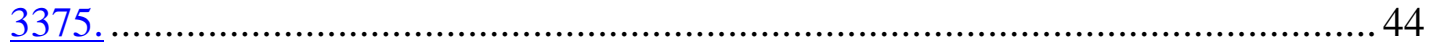

Figure 4.1.4 Class 8 Parallel HEV on CSHVC without auxiliary load, $\mathrm{C}_{1}=0.2, \mathrm{C}_{2}=$

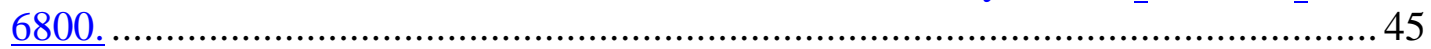

Figure 4.1.5 Class 8 Series HEV on Yard Cycle without auxiliary load, $\mathrm{C}_{1}=0.3, \mathrm{C}_{2}=$ $\underline{2550 .}$ 
Figure 4.1.6 Class 2B Parallel HEV on Test D Cycle with auxiliary load, $\mathrm{C}_{1}=0.1, \mathrm{C}_{2}=$

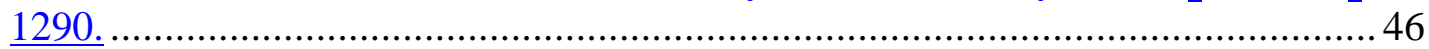

Figure 4.1.7 Class 6 Series HEV on Combined Cycle without auxiliary load, $\mathrm{C}_{1}=0.2, \mathrm{C}_{2}$

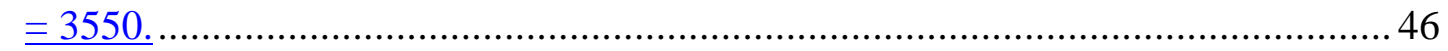

Figure 4.2.1 Class 2B HEV Fuel Economy on Freeway Cycle ...................................... 48

Figure 4.2.2 Class 6 HEV Fuel Economy on Freeway Cycle. …………......................... 48

Figure 4.2.3 Class 8 HEV Fuel Economy on Freeway Cycle. ………............................ 49

Figure 4.2.4 Class 2B HEV Fuel Economy on CSHVC Cycle. .....................................5 50

Figure 4.2.5 Class 6 HEV Fuel Economy on CSHVC Cycle...................................... 50

Figure 4.2.6 Class 8 HEV Fuel Economy on CSHVC Cycle.......................................... 51

Figure 4.2.7 Class 2B HEV Fuel Economy on Yard Cycle. ...................................... 51

Figure 4.2.8 Class 6 HEV Fuel Economy on Yard Cycle.............................................5 52

Figure 4.2.9 Class 8 HEV Fuel Economy on Yard Cycle..............................................53

Figure 4.2.10 Class 2B HEV Fuel Economy on Manhattan Cycle.................................53

Figure 4.2.11 Class $6 \mathrm{HEV}$ fuel economy on Manhattan Cycle...................................... 54

Figure 4.2.12 Class 8 HEV fuel economy on Manhattan Cycle..................................... 54

Figure 4.2.13 Class 2B HEV fuel economy on Test D Cycle. ………......................... 55

Figure 4.2.14 Class 6 HEV fuel economy on Test D Cycle......................................... 55

Figure 4.2.15 Class 8 HEV fuel economy on Test D Cycle ...........................................5 56

Figure 4.2.16 Class 2B HEV fuel economy on Combined Cycle...................................57

Figure 4.2.17 Class 6 HEV fuel economy on Combined Cycle. ......................................5 57

Figure 4.2.18 Class 8 HEV fuel economy on Combined Cycle. .....................................58

Figure 4.3.1 Power required to maintain constant speed on various grades for Class 2B.

Figure 4.3.2 Power required to maintain constant speed on various grades for Class 2B.

Figure 4.3.3 Power required to maintain constant speed on various grades for Class $8 . .64$ Figure 4.3.4 Power required for Class 6 vehicle over Freeway Cycle with and without

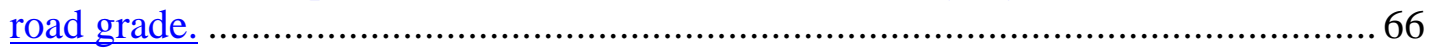




\section{Table of Tables}

Table 1.2.1 European Heavy-Duty Diesel Engine Emissions Regulations [6] …….......... 4

Table 1.2.2 US EPA Heavy-Duty Diesel Engine Emissions Regulations [6] .................... 4

Table 3.1.1 Vehicle weight classes. [7] ................................................................... 10

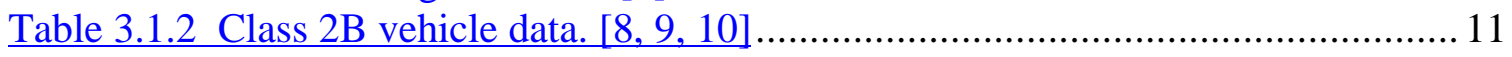

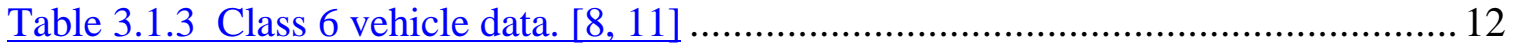

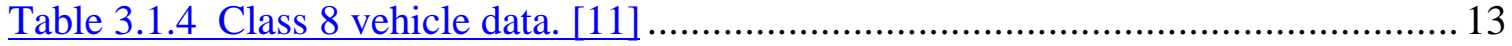

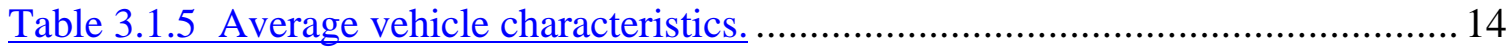

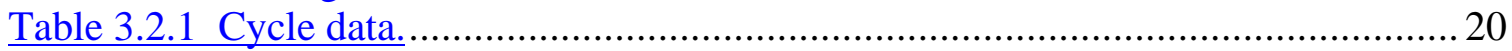

Table 3.4.1 Hawker Genesis G13EP Battery Properties. [16]........................................ 37

Table 4.1.1 Conventional Vehicle uncorrected fuel economy from simulation.............. 42

Table 4.1.2 Average Fuel Economy for In-use Conventional Vehicles [18, 19] ............ 42

Table 4.2.1 Class 2B optimum HEV without auxiliary load configurations and constants

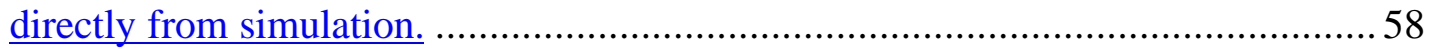

Table 4.2.2 Class 2B optimum HEV with auxiliary load configurations and constants

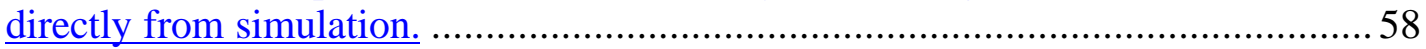

Table 4.2.3 Class 2B without auxiliary load simulation results compared to actual

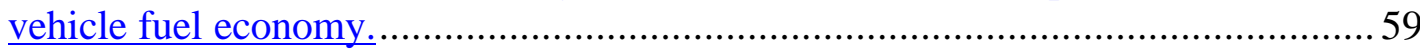

Table 4.2.4 Class 6 optimum HEV without auxiliary load configurations and constants

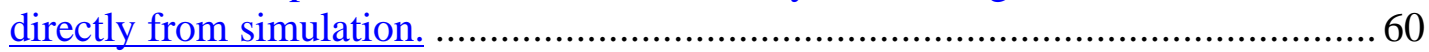

Table 4.2.5 Class 6 optimum HEV with auxiliary load configurations and constants

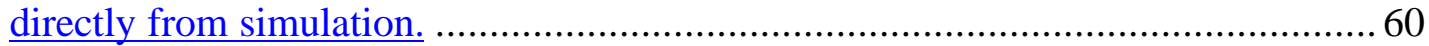

Table 4.2.6 Class 8 optimum HEV without auxiliary load configurations and constants

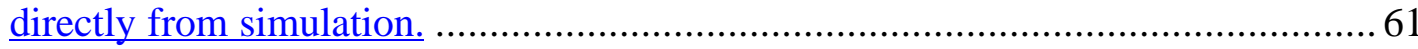

Table 4.2.7 Class 8 optimum HEV with auxiliary load configurations and constants

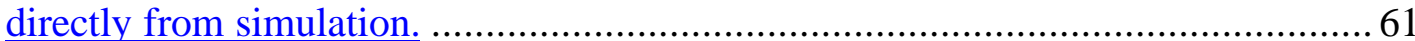

Table 4.2.8 Simulation results from reversed Combined Cycle......................................62 


\section{Nomenclature}
A
- Frontal Area
$\mathrm{C}_{\mathrm{b}}$
-Battery Energy Capacity
$\mathrm{C}_{\mathrm{D}}$
-Aerodynamic Drag Coefficient
CSHVC
-City-Suburban Heavy Vehicle Cycle
$\mathrm{E}_{\mathrm{b}}$
-Battery Energy
$\mathrm{E}_{\mathrm{bi}}$
-Battery Energy at a given time step
$\mathrm{F}$
-Force
$\mathrm{F}_{\mathrm{i}}$
-Inertial Force
$\mathrm{F}_{\mathrm{D}}$
-Aerodynamic Drag Force
$\mathrm{F}_{\text {rr }}$
-Rolling Resistance Force
$\mathrm{F}_{\mathrm{w}}$
-Force at the Wheels
$\mathrm{F}_{\grave{\mathrm{e}}}$
-Force Due to Road Grade
g
-Gravitational Acceleration
HEV
-Hybrid Electric Vehicle
HDDC
-Heavy Duty Drive Cycle
$\eta_{\mathrm{d}}$
-Driveline Efficiency
$\eta_{\mathrm{b}}$
-Battery Efficiency
ICE
-Internal Combustion Engine
$\mathrm{I}_{\mathrm{b}}$
-Battery Current
$\mathrm{m}$
- Mass
$\mu$
-Rolling Resistance Constant
$\mathrm{N}_{\mathrm{b}}$
-Number of Batteries 


$\begin{array}{ll}\text { NMHC } & \text {-Non-methane hydrocarbons } \\ \text { OTR } & \text {-Over-the-Road } \\ \mathrm{P}_{\mathrm{bi}} & \text {-Battery Power at a given time-step } \\ \mathrm{P}_{\mathrm{e}} & \text {-Engine Power } \\ \mathrm{P}_{\mathrm{w}} & \text {-Power at the Wheels } \\ \mathrm{P}_{\mathrm{aux}} & \text {-Auxiliary Load Power } \\ \rho & \text {-Air Density } \\ \text { SoC } & \text {-State of Charge } \\ \mathrm{SoC}_{\mathrm{i}} & \text {-State of Charge at a given time-step } \\ \text { SoC }_{t} & \text {-Target State of Charge } \\ \theta & \text {-Road Grade Angle } \\ \mathrm{V} & \text {-Velocity } \\ \mathrm{V}_{\mathrm{b}} & \text {-Nominal Battery Voltage }\end{array}$




\section{Introduction}

In recent years, rising fuel costs, declining oil reserves, and increased concern over environmental issues has led to government and public interest in the design of new, more efficient means of motor vehicle transportation. While existing technology has improved the fuel economy and tailpipe emissions of vehicles far beyond standards from only a decade or two ago, further advances are both desired and required. Concern over these issues led the government and the automotive industry to form Partnership for a New Generation of Vehicles (PNGV). PNGV calls for the production of vehicles with three times the fuel economy of current conventional vehicles. [1] The $21^{\text {st }}$ Century Truck Program is a partnership between the heavy-duty truck and bus industry and the federal government for the development of technology that will dramatically reduce their emissions and increase their fuel economy, doubling the fuel economy of Class 8 trucks and tripling the fuel economy of Class 2B and 6 trucks by 2010 as well as decreasing emissions and increasing safety. [2]

One method for increasing the fuel economy of vehicles of all sizes is the use of hybridization. In a hybrid electric vehicle (HEV), two energy sources are used to power the vehicle. One source is an electric motor supported by some type of energy storage device such as batteries, ultra capacitors, or flywheels. The other source has ranged from an internal combustion engine (ICE), to a gas turbine, or fuel cell. While ultra capacitors, flywheels, and fuel cells hold promise for the future, they have not reached a level of availability, reliability, and cost that would allow them to go into mass production in the near future. In this thesis, the HEVs simulated are assumed to be powered by a 
conventional diesel ICE, electric motor(s), and batteries. HEVs have several advantages over conventional vehicles. The largest factor in their increased fuel economy is the ability to capture braking energy, often referred to as regenerative braking energy. During braking in a conventional vehicle, the kinetic energy associated with the mass of the vehicle at a given speed is dissipated as heat through friction between the brake pads and rotors or drums to slow the vehicle. This energy is lost and cannot be reused. In an $\mathrm{HEV}$, the electric motor is used to decelerate the vehicle generating power, which is stored in batteries and used to accelerate the vehicle. Some of the energy from the fuel used to accelerate the vehicle can be captured during deceleration and reused for subsequent acceleration. HEVs also allow downsizing of the ICE in most vehicles. The ICE in a conventional vehicle is sized to provide the peak power necessary to provide dynamic performance that is acceptable to the consumer. This peak power is seldom used and the engine often operates at low load and poor efficiency. In an HEV, peak power is provided by supplementing the ICE power with electric power allowing average engine operation to be closer to the optimum range increasing efficiency and often decreasing the weight of the vehicle.

\subsection{Literature Review}

Hybrid electric vehicles are not a new concept. The first vehicle powerplants were steam engines, internal combustion engines, and electric motors. The first electric vehicle was made by the Dutch Professor Stratingh in 1835. This was followed by several other designs until, in 1899, advances in motor and battery design allowed Baker Electric of the US to manufacture an electric vehicle capable of a maximum speed of 40 $\mathrm{km} / \mathrm{h}$ and an $80 \mathrm{~km}$ range. During this same period, many advances were made in 
internal combustion engine technology. The two technologies were combined to provide the increased range of an ICE powered vehicle with the safety and reliability of an electric vehicle. Increasingly powerful and reliable ICEs eventually replaced the widespread use of electric motors. [3]

Recent years have seen a resurgence in the popularity of HEVs both in light and heavy-duty applications. Examples of current production HEVs include light-duty vehicles such as the Toyota Prius and Honda Insight, and heavy-duty vehicles such as Orion/Lockheed transit buses. These vehicles have demonstrated the advantages of HEVs to the public and they can only be expected to become more popular.

The most common current use for heavy-duty HEVs is in the transit bus industry. In-use data from fleets in New York City have demonstrated $30-50 \%$ gains in fuel economy as well as $50-90 \%$ lower $\mathrm{PM}, 30-60 \%$ lower $\mathrm{NO}_{\mathrm{x}}$ and $\mathrm{HC}$, and $20-40 \%$ lower greenhouse gases. [4]

Using current technology, hybrid electric powertrains are quite expensive, but as consumer interest grows, increased production levels and research and development funding should drive the prices down. The cost of integrating a parallel HEV powertrain into an existing Class 3 vehicle is estimated at $\$ 5,800$ in 2005 but drops to $\$ 3,000$ in 2020. Similarly, for Class 6 - 7 vehicles, the 2005 cost is $\$ 7,100$ dropping to $\$ 3,300$ in 2020. [5]

\subsection{Emissions Regulations}

Current and future emissions regulations for heavy-duty vehicles vary between those mandated by the US federal government, the California Air Resources Board (CARB), 
and Europe but, they all mandate substantial decreases in the vehicle emissions over the next 5 to 7 years.

Table 1.2.1 European Heavy-Duty Diesel Engine Emissions Regulations [6]

\begin{tabular}{|c|c|c|c|c|c|}
\cline { 2 - 6 } \multicolumn{1}{c|}{} & Year & $\mathrm{CO}$ & $\mathrm{HC}$ & $\mathrm{NOx}$ & $\mathrm{PM}$ \\
\cline { 2 - 6 } \multicolumn{1}{c|}{} & & $\mathrm{g} / \mathrm{kWh}$ & $\mathrm{g} / \mathrm{kWh}$ & $\mathrm{g} / \mathrm{kWh}$ & $\mathrm{g} / \mathrm{kWh}$ \\
\hline Euro I & $1992,<85 \mathrm{~kW}$ & 4.5 & 1.1 & 8.0 & 0.612 \\
\hline & $1992,>85 \mathrm{~kW}$ & 4.5 & 1.1 & 8.0 & 0.36 \\
\hline Euro II & 1996 & 4.0 & 1.1 & 7.0 & 0.25 \\
\hline & 1998 & 4.0 & 1.1 & 7.0 & 0.15 \\
\hline Euro III & 2000 & 2.1 & 0.66 & 5.0 & 0.10 \\
\hline Euro IV & 2005 & 1.5 & 0.46 & 3.5 & 0.02 \\
\hline Euro V & 2008 & 1.5 & 0.46 & 2.0 & 0.02 \\
\hline
\end{tabular}

Table 1.2.2 US EPA Heavy-Duty Diesel Engine Emissions Regulations [6]

\begin{tabular}{|c|c|c|c|c|}
\hline Year & $\mathrm{CO}$ & $\mathrm{HC}$ & NOx & PM \\
\hline & $\mathrm{g} / \mathrm{bhp}-\mathrm{hr}$ & g/bhp-hr & g/bhp-hr & $\mathrm{g} / \mathrm{bhp}-\mathrm{hr}$ \\
\hline \multicolumn{5}{|c|}{ Heavy-Duty Diesel Truck Engines } \\
\hline 1990 & 1.3 & 15.5 & 6.0 & 0.60 \\
\hline 1991 & 1.3 & 15.5 & 5.0 & 0.25 \\
\hline 1994 & 1.3 & 15.5 & 5.0 & 0.10 \\
\hline 1998 & 1.3 & 15.5 & 4.0 & 0.10 \\
\hline 2000 & & & 4.0 & 0.05 \\
\hline 2002 & & & $2.5^{*}$ & 0.01 \\
\hline 2004 & & & 0.5 & 0.01 \\
\hline 2007 & & & 0.2 & 0.01 \\
\hline \multicolumn{5}{|c|}{ Urban Bus Engines } \\
\hline 1991 & 1.3 & 15.5 & 5.0 & 0.25 \\
\hline 1993 & 1.3 & 15.5 & 5.0 & 0.10 \\
\hline 1994 & 1.3 & 15.5 & 5.0 & 0.07 \\
\hline 1996 & 1.3 & 15.5 & 5.0 & 0.05 \\
\hline 1998 & 1.3 & 15.5 & 4.0 & 0.05 \\
\hline 2000 & & & 4.0 & 0.05 \\
\hline 2002 & & & $2.5^{*}$ & 0.01 \\
\hline 2004 & & & 0.5 & 0.01 \\
\hline 2007 & & & 0.2 & 0.01 \\
\hline
\end{tabular}

$*$ NOx + NMHC 


\section{Vehicle Configurations}

Three basic vehicle configurations were considered. These included a conventional ICE powered vehicle, a series HEV, and a parallel HEV.

\subsection{Conventional Vehicle}

In a conventional vehicle, an ICE transmits the power needed to drive the vehicle through a transmission and differential to the wheels. This layout has been used for many years and is fairly inexpensive and easy to control. The main disadvantage is poor efficiency due to the use of an oversized ICE, lack of regenerative braking, and highly transient ICE operation. The transient engine operation also leads to the increased production of emissions and difficulty in controlling them.

\subsection{Electric Vehicle}

The use of pure electric vehicles is older, in fact, than the use of the ICE. In this type of vehicle, electrical energy is stored in a battery and fed to the motor, which provides power to the wheels.

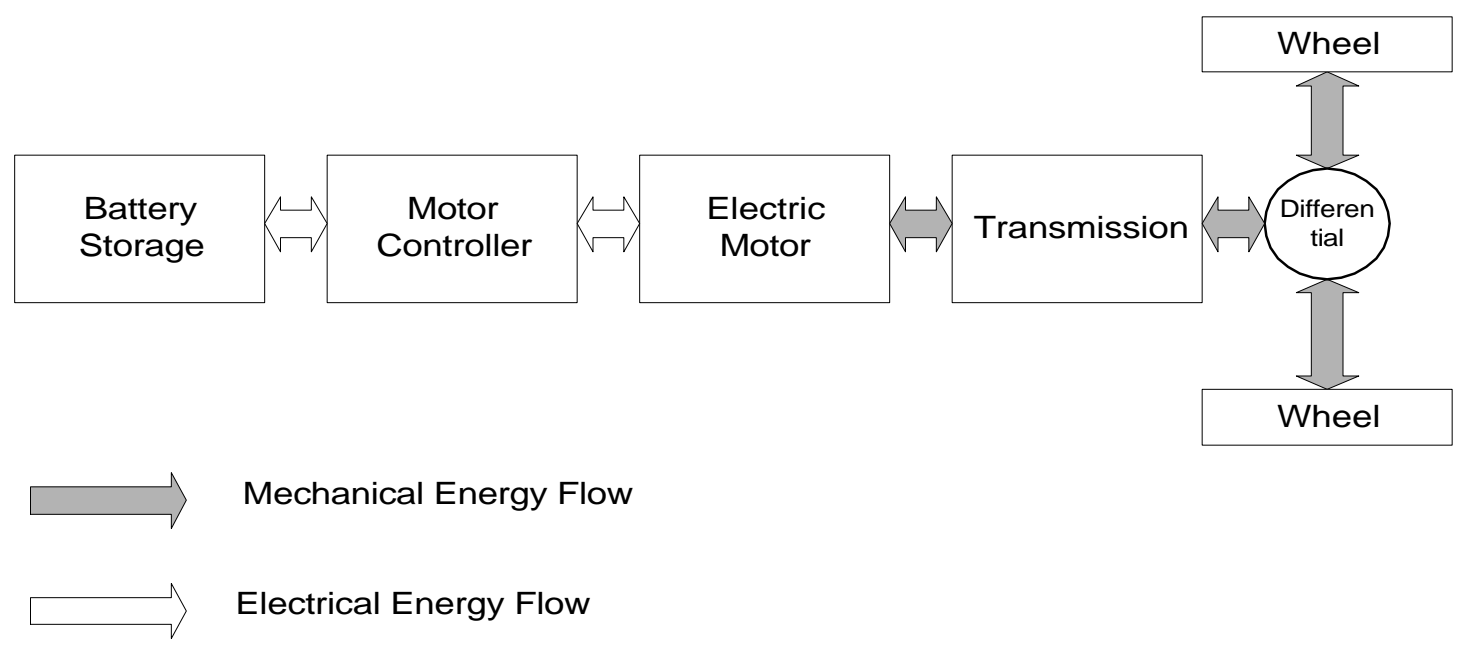

Figure 2.2.1 Electric vehicle layout. [3] 
The advantages of electric vehicles include zero tailpipe emissions and nearly silent operation compared to most conventional vehicles.

Disadvantages come in the form of reduced consumer acceptability due to short range and long recharge times. Current production EVs are limited to a maximum of approximately 150 miles on one battery charge. Use of air conditioning or heating increases the demands on the batteries further reducing this range. While this is sufficient for many daily commuters, it is not ideal for long trips. In addition to this short range, the batteries require several hours to recharge once depleted. Deep cycling of batteries causes damage reducing their lifetime and requiring expensive, periodic replacement. The idea of not being able to use a vehicle for several hours out of every day is unacceptable to many consumers.

\subsection{Series HEV}

In a series $\mathrm{HEV}$, an ICE or fuel cell is used to produce electrical energy that is sent to the battery pack and electric motor. All of the power to drive the vehicle is supplied through the electric motor. Figure 2.3.1 shows a typical series HEV layout. 


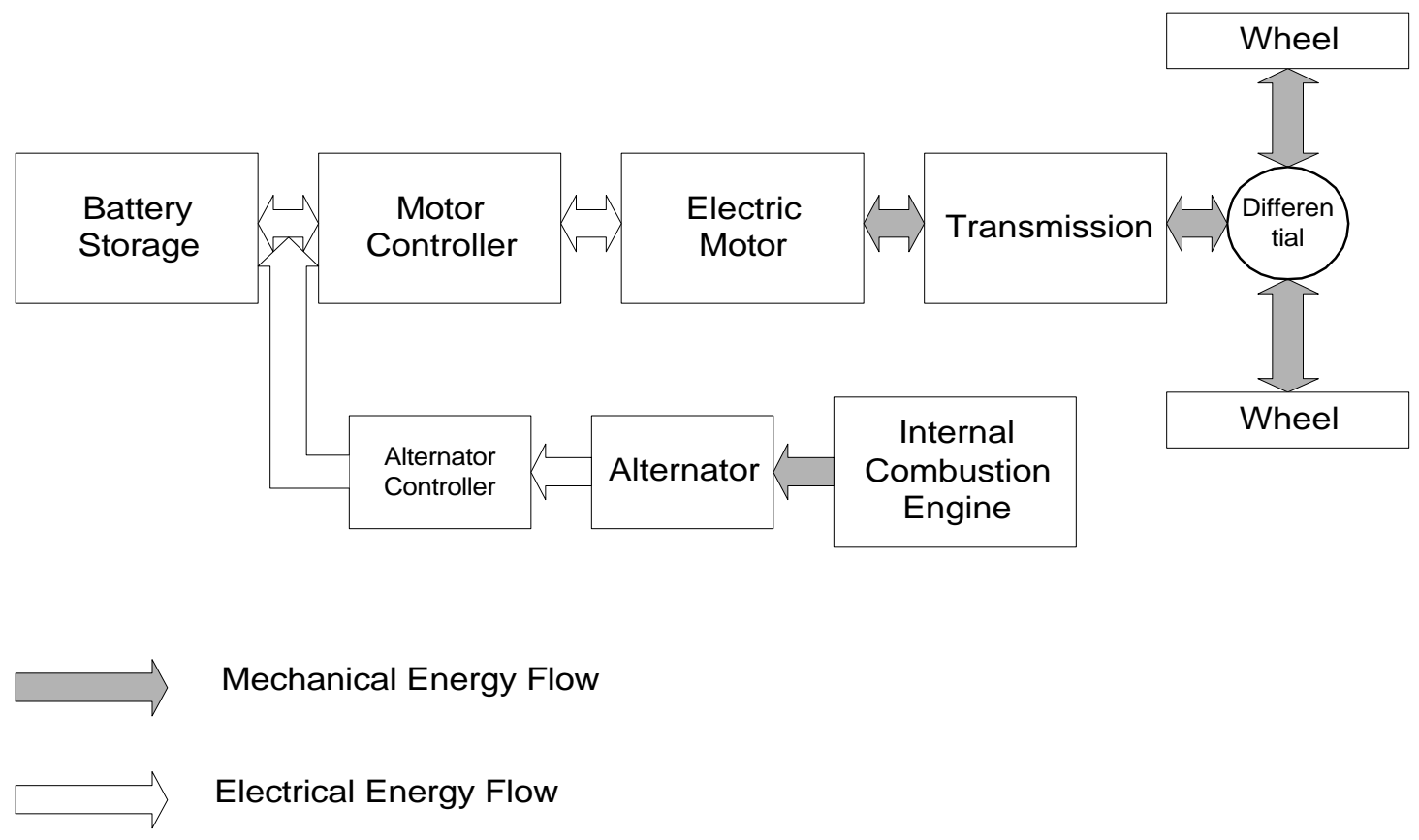

\section{Figure 2.3.1 Series HEV layout. [3]}

Some of the advantages of series HEVs include less transient ICE operation, operation of the ICE at optimal, efficient speed and load for improved fuel economy and optimization of emissions control, and the possible omission of the costly, heavy transmission. Disadvantages in current series HEVs include poor dynamic performance, losses during changing energy from chemical to mechanical, mechanical to electrical, and electrical to mechanical forms, and the need for costly, heavy battery packs and electric motors.

Series vehicles typically show substantial fuel economy improvements in highly transient driving in urban situations due to recovery of large amounts of regenerative braking energy. Smaller efficiency gains are realized through less transient operation such as highway driving where there is less available regenerative braking energy. 


\subsection{Parallel HEV}

In a parallel HEV, there is a direct connection between both the ICE and the electric motor and the wheels. This configuration allows a wide variety of control strategies to be employed. When high power is demanded such as for high acceleration, both the ICE and electric motor deliver power to the wheels. In less demanding situations, the ICE can be operated at a higher power that what is required to drive the vehicle and the excess power captured by the electric motor and stored in the batteries for later use, or the electric motor alone can be used to drive the vehicle. This has the advantage of operating the ICE in a more efficient mode or not at all. During long, steady-state cruises, the ICE engine alone can drive the vehicle avoiding the inherent inefficiency of the batteries.

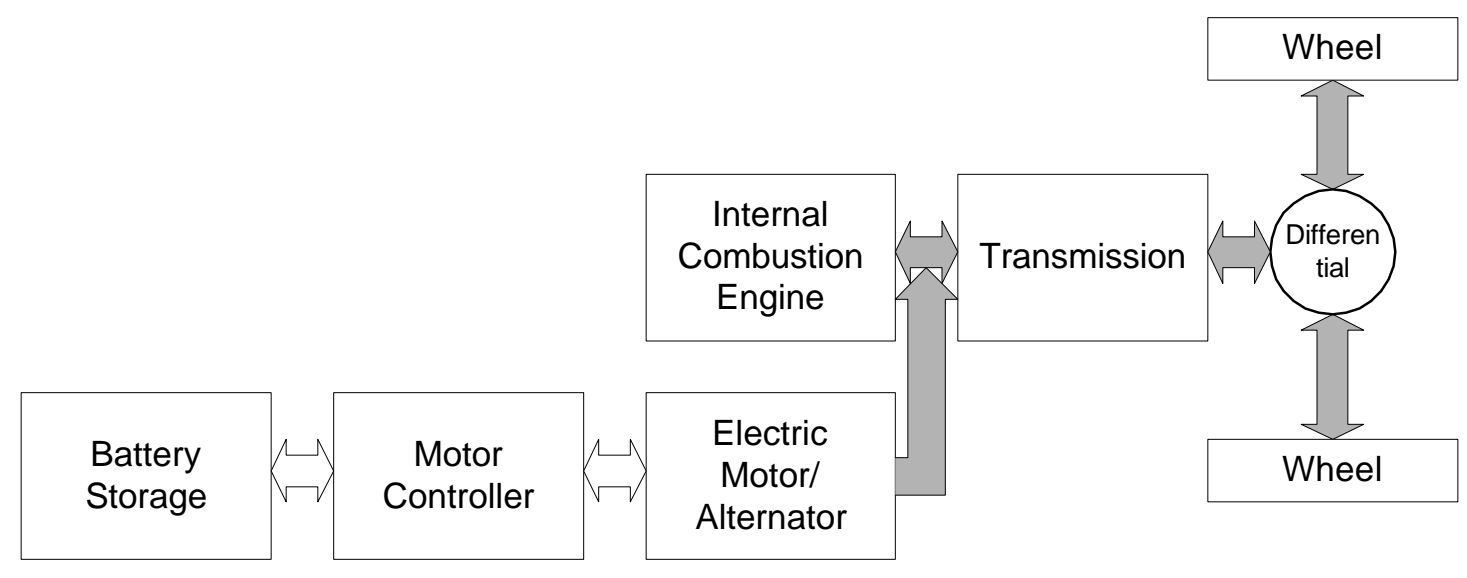

Mechanical Energy Flow

Electrical Energy Flow

Figure 2.4.1 Parallel HEV layout with electric power delivered between ICE and transmission. [3] 
The main advantage of parallel HEVs is improved dynamic performance compared to series HEVs due to the direct coupling between the ICE, electric motor, and the wheels.

This comes with disadvantages. Since the ICE is directly coupled to the wheels, it is forced into more transient operation than the ICE in a series vehicle. This tends to result in poorer efficiency and increased emissions. 


\section{Simulation}

To examine and optimize the various vehicle configurations, they were, first, simulated using a Microsoft Excel based simulation.

\subsection{Vehicle Descriptions}

Three vehicle weight classes were chosen for simulation. Class 2B vehicles include 1 ton trucks such as GM's 3500 series or Ford's F350. Typical Class 6 vehicles include local delivery box trucks and school buses. There are a wide variety of Class 8 vehicles but those from the WVU database are primarily over-the-road (OTR) tractors and city buses.

Table 3.1.1 Vehicle weight classes. [7]

\begin{tabular}{|c|c|c|c|c|c|c|}
\cline { 2 - 7 } \multicolumn{1}{c|}{} & Class & Min GVW & Max GVW & & Min GVW & Max GVW \\
\cline { 2 - 7 } \multicolumn{1}{c|}{} & & $\mathrm{lb}$ & $\mathrm{lb}$ & & $\mathrm{kg}$ & $\mathrm{kg}$ \\
\cline { 2 - 7 } & & & & & & \\
\hline \multirow{3}{*}{ Light Duty } & 1 & & $<6000$ & & 0 & $<2721$ \\
\cline { 2 - 7 } & $2 \mathrm{~A}$ & 6001 & 8500 & & 2722 & 3856 \\
\cline { 2 - 7 } & $2 \mathrm{~B}$ & 8501 & 10000 & & 3856 & 4536 \\
\cline { 2 - 7 } & 3 & 10001 & 14000 & & 4536 & 6350 \\
\hline \multirow{3}{*}{ Heavy Duty } & 4 & 14001 & 16000 & & 6351 & 7258 \\
\cline { 2 - 7 } & 5 & 16001 & 19500 & & 7258 & 8845 \\
\cline { 2 - 7 } & 6 & 19501 & 26000 & & 8846 & 11794 \\
\cline { 2 - 7 } & 8 & 26001 & 33000 & & 11794 & 14969 \\
\hline
\end{tabular}

To simulate a vehicle from each of the three weight classes, an average vehicle was calculated using data from vehicle and engine manufacturers and from vehicles tested on the WVU Transportable Heavy-Duty Emissions Testing Laboratory. To avoid requiring a vehicle to meet the high instantaneous power demands encountered in some driving cycles, an average maximum power was calculated for each vehicle class. In any driving situation requiring high power, a vehicle would be expected to operate 
somewhere between its maximum rated power and its maximum torque point. Due to this, maximum power given to the averaged vehicle was the average of both the maximum rated power and the power at maximum torque.

The average Class 2B vehicle was calculated from manufacturer data for empty trucks with an added $1500 \mathrm{~kg}$ payload. Class 6 data is from both manufacturer data and the WVU database. Class 8 data is taken solely from the WVU database.

Table 3.1.2 Class 2B vehicle data. $[8,9,10]$

\begin{tabular}{|c|c|c|c|c|}
\hline Vehicle & Engine & Mass & Max Power & Power @ Max Torque \\
\hline & & $\mathrm{kg}$ & $\mathrm{kW}$ & $\mathrm{kW}$ \\
\hline GMC 3500HD & 6.6L diesel & 4024 & 224 & 133 \\
\hline Dodge 3500 & 5.9L diesel & 3932 & 183 & 115 \\
\hline Ford F350 Super Duty & $7.3 \mathrm{~L}$ diesel & 3910 & 205 & 119 \\
\hline
\end{tabular}

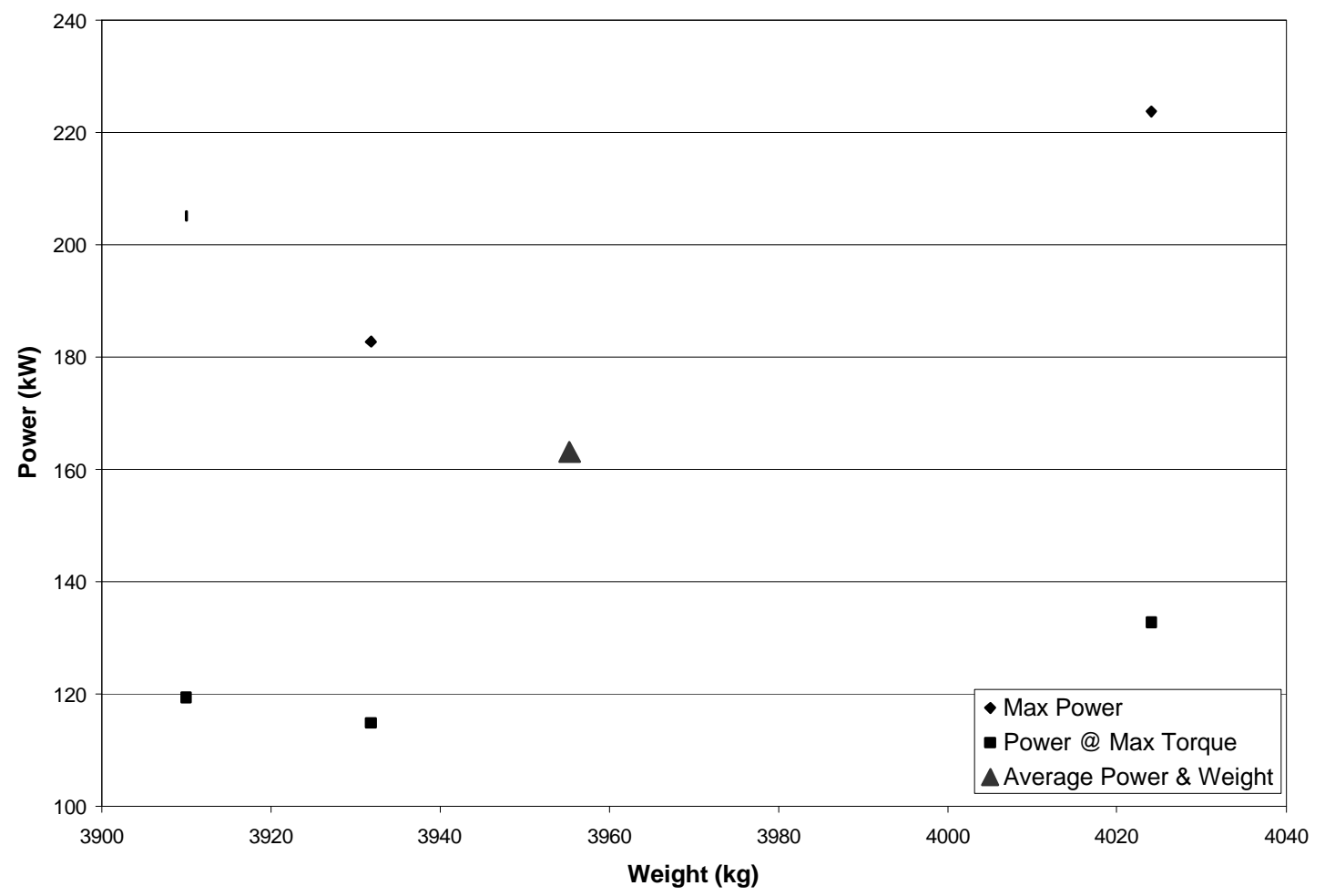

Figure 3.1.1 Class 2B vehicle power and weight. 
Table 3.1.3 Class 6 vehicle data. $[8,11]$

\begin{tabular}{|c|c|c|c|c|}
\hline Vehicle & Engine & Weight & Max Power & Power @ Max Torque \\
\hline & & $\mathrm{kg}$ & $\mathrm{kW}$ & $\mathrm{kW}$ \\
\hline GMC T6500 & 7.8L diesel & 4545 & 111 & 70 \\
\hline GMC T6500 & 7.8L diesel & 5165 & 128 & 81 \\
\hline GMC T6500 & 7.2L diesel & 4545 & 117 & 80 \\
\hline GMC T6500 & 7.2L diesel & 5165 & 139 & 101 \\
\hline Chevrolet & Caterpillar 3116 & 5362 & 103 & 86 \\
\hline International & International DTA3600 & 4917 & 100 & 75 \\
\hline GMC & Caterpillar 3208 & 4545 & 95 & 70 \\
\hline Mack/Renault & Renault MIDR & 5269 & 106 & 66 \\
\hline Ford & LFM078EPC7 & 5062 & 92 & 61 \\
\hline
\end{tabular}

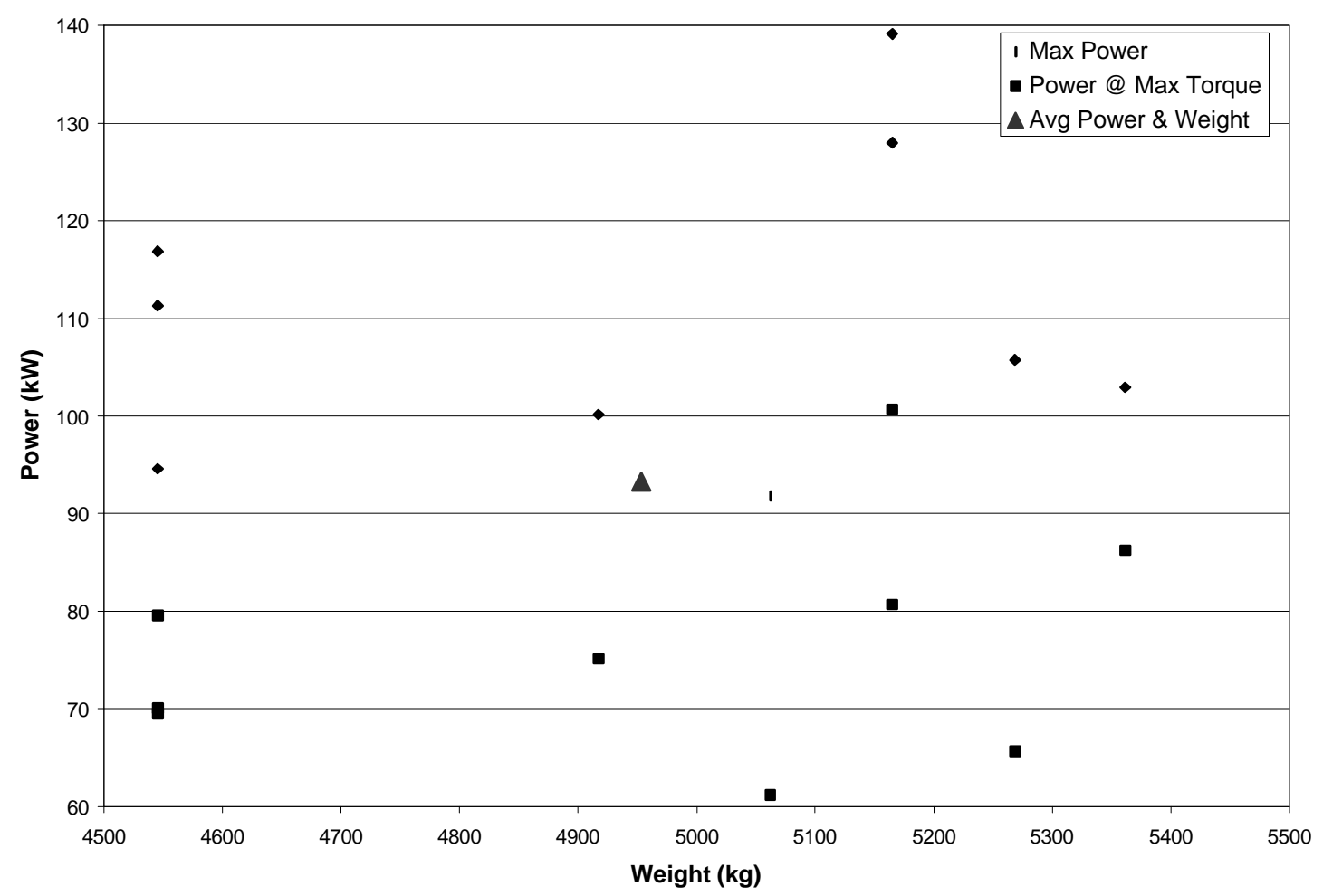

Figure 3.1.2 Class 6 vehicle power and weight. 
Table 3.1.4 Class 8 vehicle data. [11]

\begin{tabular}{|c|c|c|c|c|}
\hline Vehicle & Engine & Weight & Max Power & Power @ Max Torque \\
\hline & & $\mathrm{kg}$ & $\mathrm{kW}$ & $\mathrm{kW}$ \\
\hline Neoplan & DDC 6V-92TA & 17127 & 154 & 120 \\
\hline Orion & Cummins L-10 & 17688 & 134 & 119 \\
\hline General Motors Corp. & DDC 6V-92TA & 16773 & 141 & 124 \\
\hline Transit Motor Corp. & DDC 6V-92TA & 16773 & 141 & 124 \\
\hline Navistar & DDC 6V-92TA & 25009 & 154 & 112 \\
\hline Freightliner & Caterpillar 3406B & 36364 & 195 & 159 \\
\hline White/General Motor Corp. & DDC 6V-92TA & 36364 & 167 & 124 \\
\hline Mack & Mack E-6 & 36364 & 195 & 157 \\
\hline International & DDC Series 60 & 36364 & 195 & 172 \\
\hline Ford & Cummins 350 & 36364 & 195 & 172 \\
\hline Grumman & DDC Series 30 & 14318 & 117 & 77 \\
\hline Blue Bird & Cummins L-10 & 36364 & 134 & 119 \\
\hline Kenworth & Cummins M11-330E & 36364 & 184 & 134 \\
\hline Thomas Built & Cummins C8.3-250G & 17000 & 139 & 191 \\
\hline Sterling & DDC Series 60 & 36364 & 262 & 197 \\
\hline
\end{tabular}

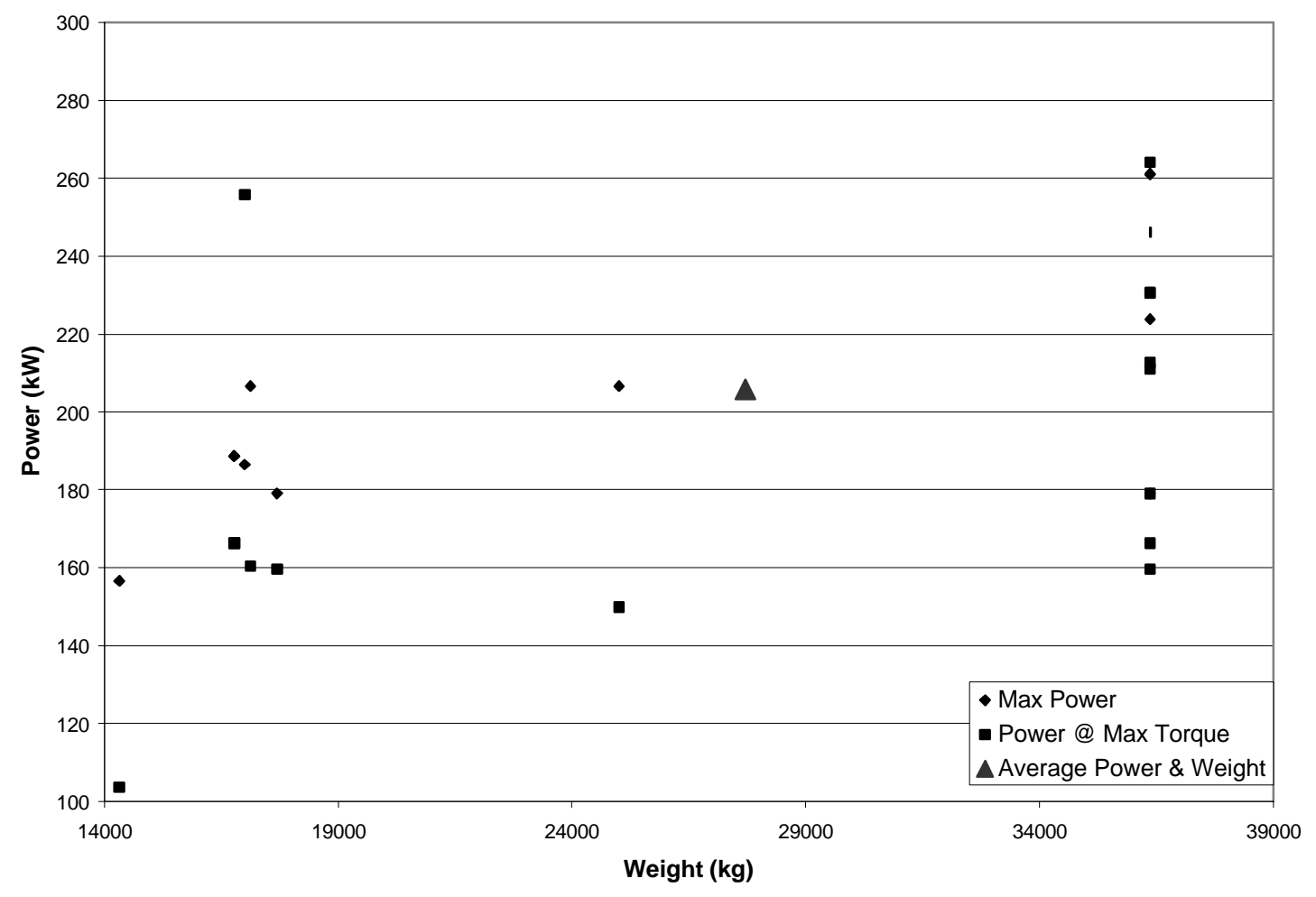

Figure 3.1.3 Class 8 vehicle power and weight.

The vehicle data in Figure 3.1.3 shows two separate groupings of vehicle weights and power ratings. The heavier group is composed of OTR trucks at maximum GVW 
while the lighter group is composed primarily of city buses. Although this results in an average vehicle that is an average for neither of these groups, it should be sufficient for this simulation.

Table 3.1.5 Average vehicle characteristics.

\begin{tabular}{|c|c|c|c|c|c|}
\hline Class & Mass & Drag Coefficient & Frontal area & Rolling resistance & Average Power \\
\hline & $\mathrm{kg}$ & & $\mathrm{m}^{\wedge} 2$ & & $\mathrm{~kW}$ \\
\hline 1 Ton & 3956 & 0.44 & 4.54 & 0.015 & 165 \\
\hline Class 6 & 10907 & 0.62 & 8.17 & 0.01 & 125 \\
\hline Class 8 & 27734 & 0.75 & 8.31 & 0.01 & 206 \\
\hline
\end{tabular}

A driver expects different levels of dynamic performance from different vehicle classes. A Class $2 \mathrm{~B}$ truck is expected to accelerate at rates similar to light-duty vehicles. $0-100 \mathrm{~km} / \mathrm{h}$ acceleration times on the order of 15 seconds are expected. For a fully loaded Class 8 over-the-road tractor, $0-100 \mathrm{~km} / \mathrm{h}$ times of 4 minutes are not unusual. These expectations are reflected in Figure 3.1.4. 


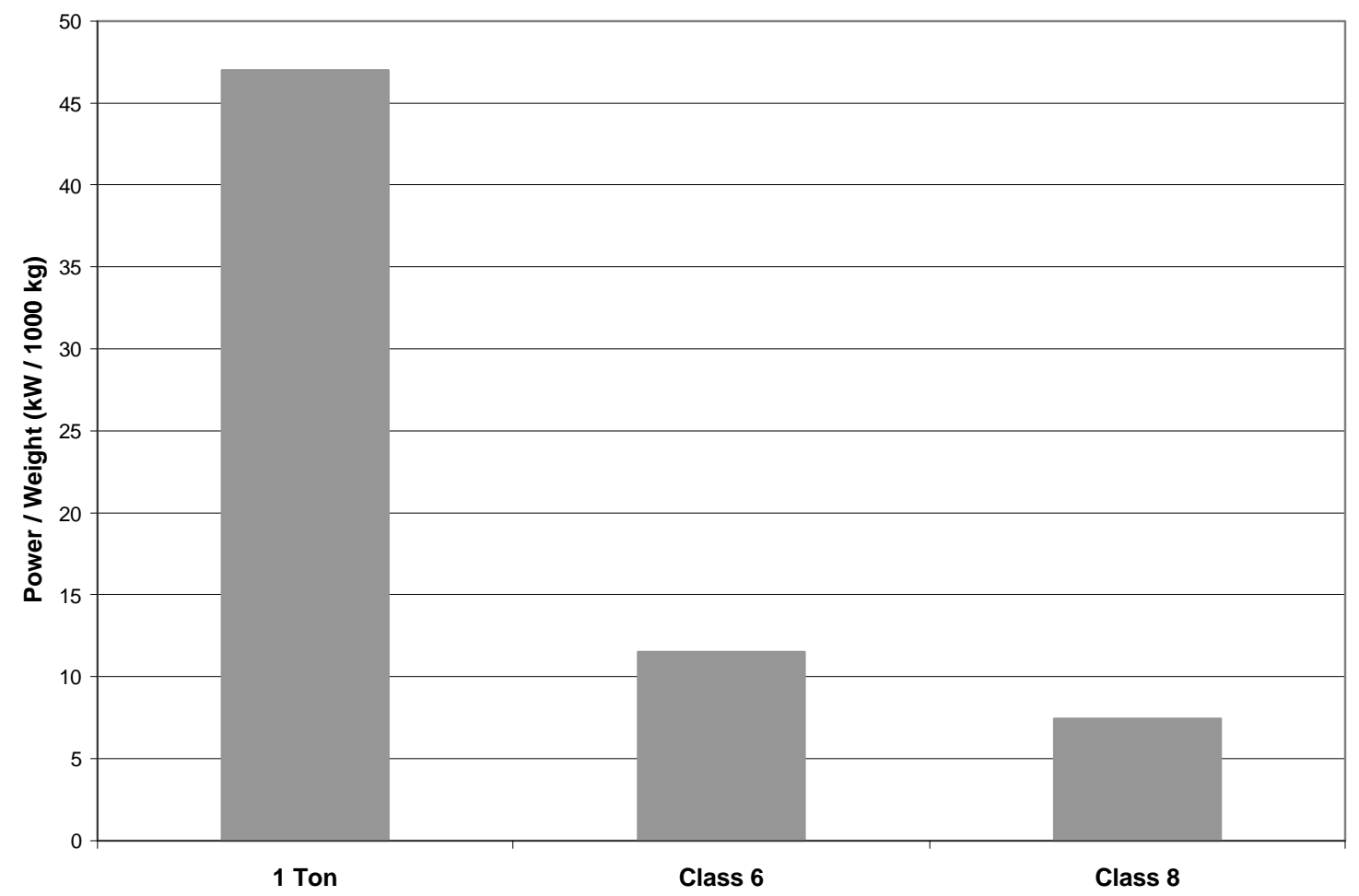

Figure 3.1.4 Typical Power / Weight ratios for various vehicle classes.

\subsection{Drive Cycles}

The three vehicles were simulated over the following speed - time traces. The traces were chosen to represent a wide variety of driving situations from low speed, highly transient urban routes to high-speed highway scenarios.

The Freeway, City-Suburban Heavy Vehicle Cycle (CSHVC), and Yard Cycles in Figures 3.2.1 to 3.2.3 were developed from examination of actual driving scenarios. The Freeway Cycle includes data from travel on four lane highways including entrance and exit ramps. The CSHVC Cycle is composed of data taken from travel in dense traffic with stoplights as well as delivery routes on the outskirts of cities. The Yard Cycle data involves trips that included changing trailers, changing tires, and driving to fueling sites. [12] 


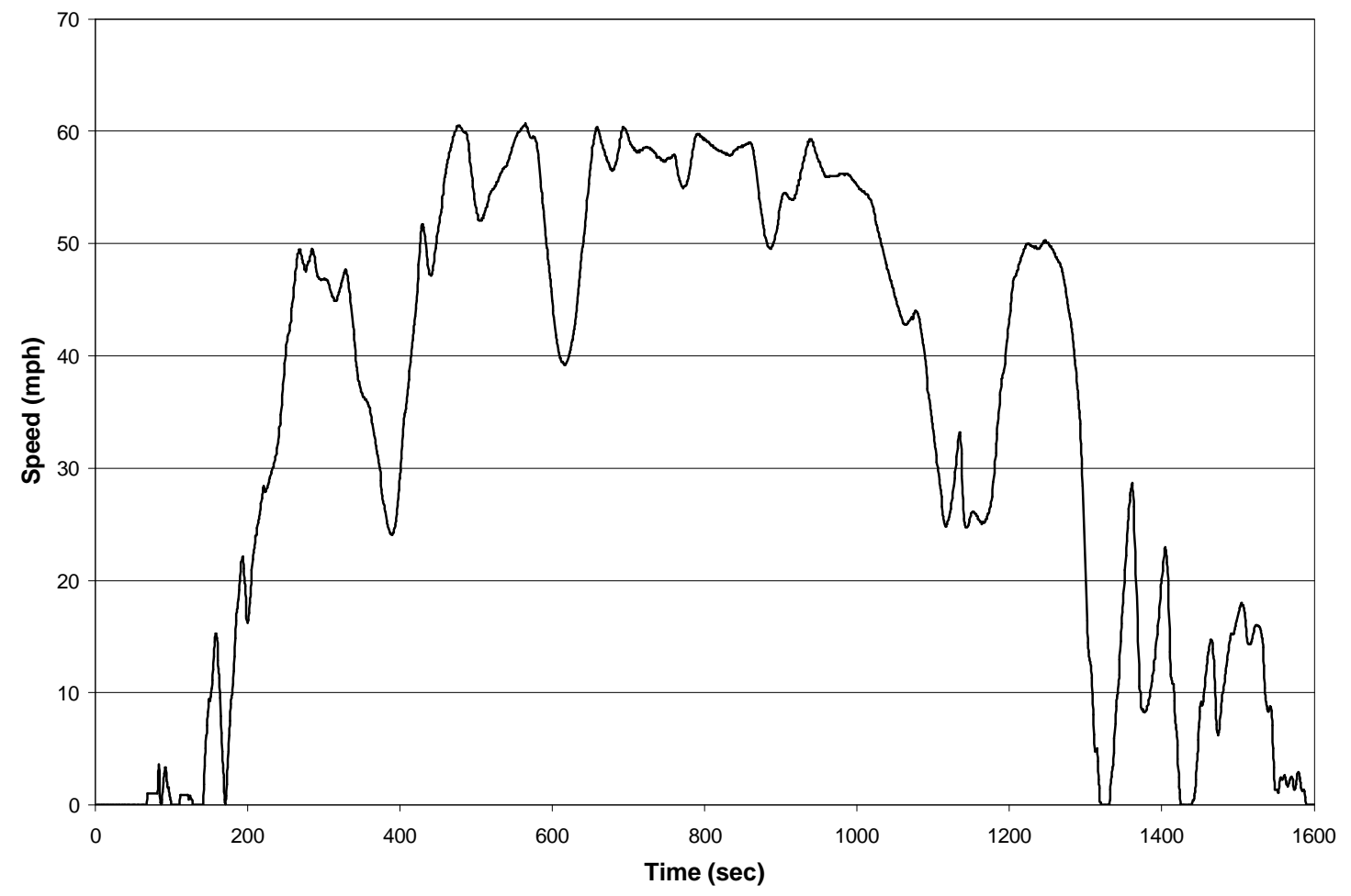

Figure 3.2.1 Speed - Time trace for the Freeway Cycle.

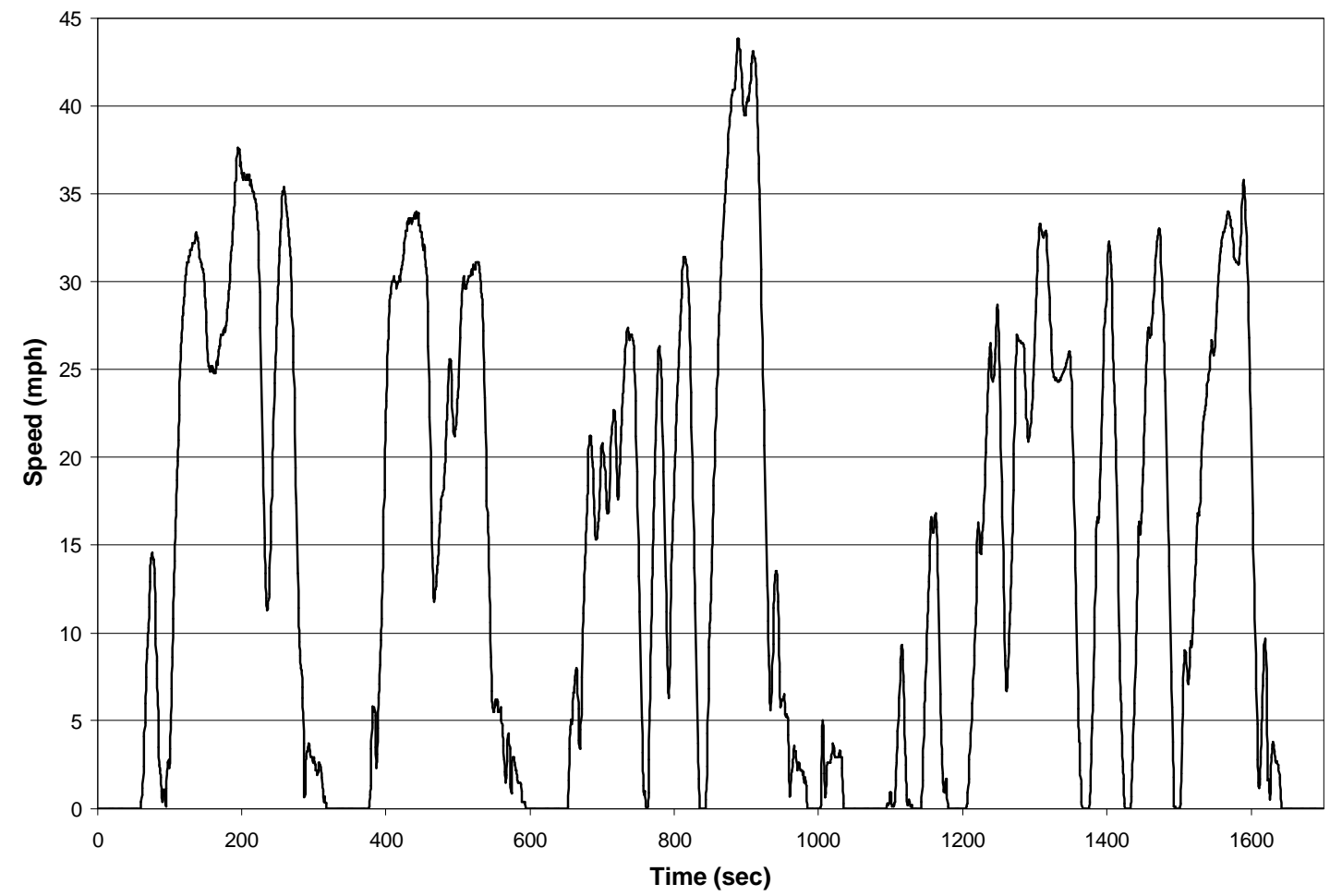

Figure 3.2.2 Speed - Time trace for the CSHVC. 


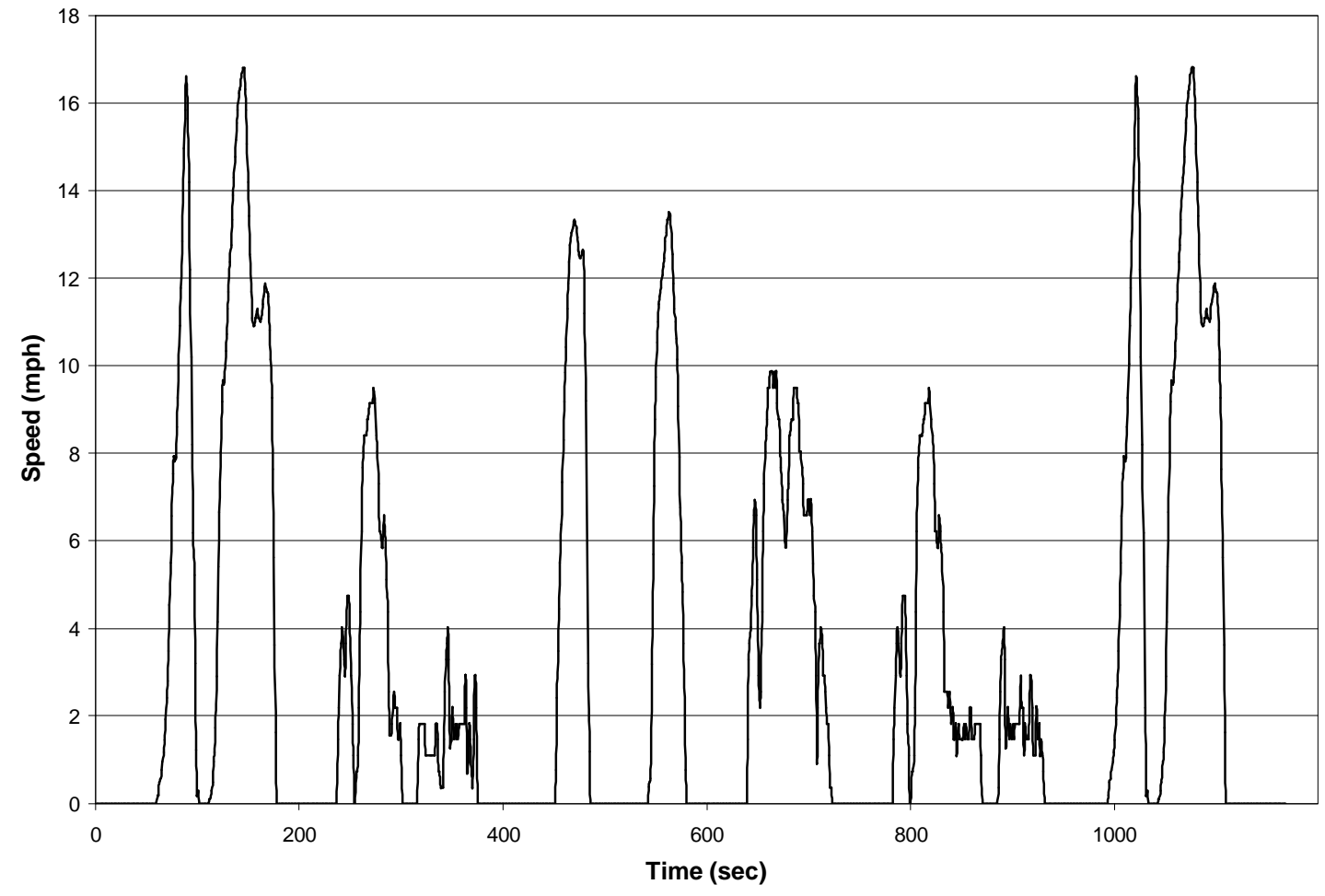

Figure 3.2.3 Speed - Time trace for the Yard Cycle.

The Manhattan Cycle was developed from actual in-use conventional and hybridelectric transit bus operation in Manhattan. The data was divided into micro-trips consisting of a start from idle, acceleration to speed, and deceleration back to idle. The data set included 399 of these micro-trips. A computer program randomly combined five micro-trips from the hybrid-electric bus data and five micro-trips from the conventional bus data and compared the statistical makeup of the created cycle to the overall data. The combination that most closely matched the overall data was chosen. [13] 


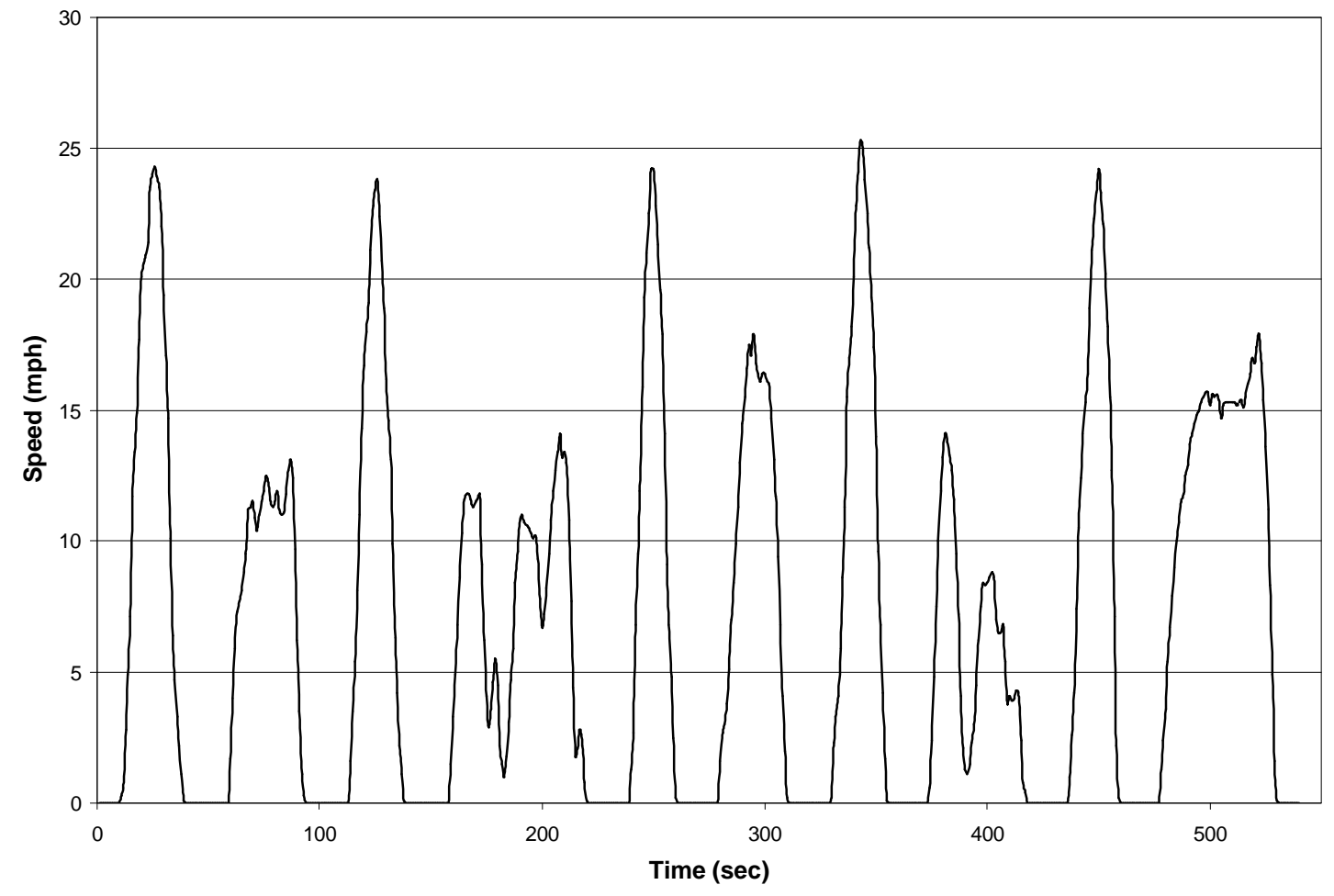

Figure 3.2.4 Speed - Time trace for the Manhattan Cycle.

Test D, also known as the 'EPA urban dynamometer driving cycle for heavy trucks' or UDDC, was developed using data logged from buses, trucks, and tractortrailers operating in New York and Los Angeles under both freeway and non-freeway conditions. A Monte Carlo simulation was then used to produce the cycle. [14] 


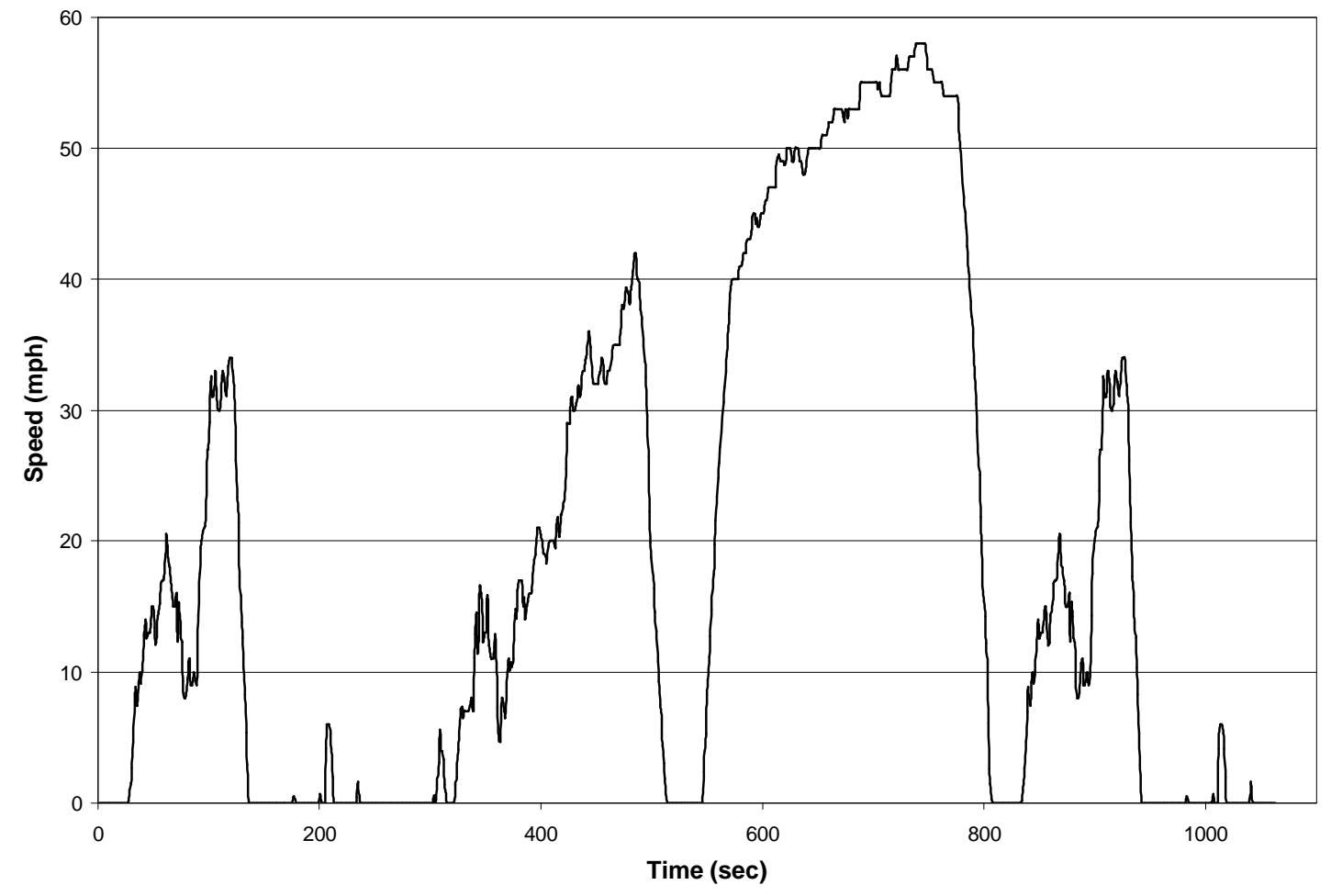

Figure 3.2.5 Speed - Time trace for the Test D Cycle.

To simulate the vehicles over a cycle more representative of a wide variety of driving situations, the five previous cycles were combined to form one, continuous speedtime trace. The ordering was: Yard, Manhattan, CSHVC, Freeway, and Test D. It will be referred to as the Combined Cycle. 


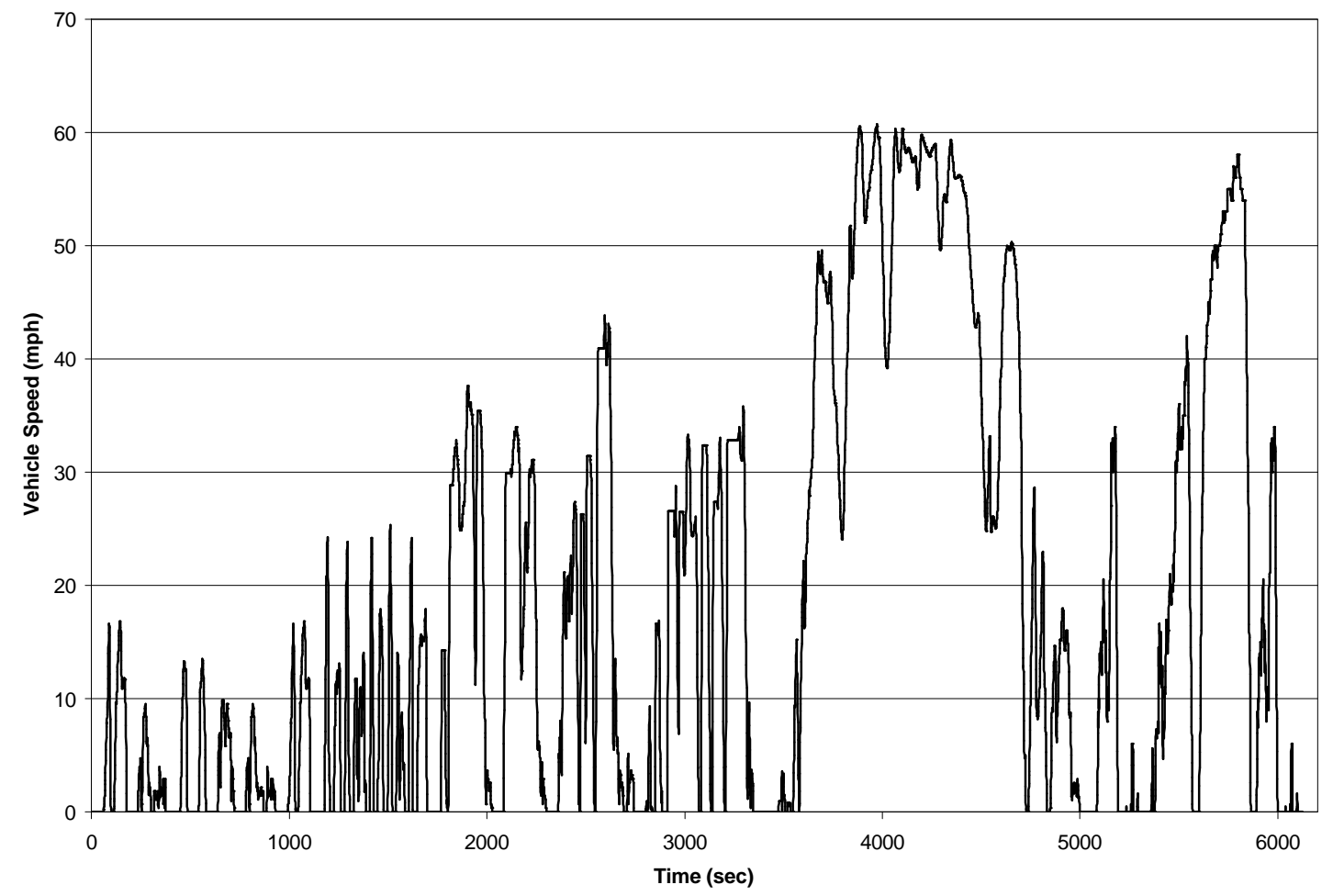

Figure 3.2.6 Speed - Time trace for the Combined Cycle.

Table 3.2.1 Cycle data.

\begin{tabular}{|c|c|c|c|c|c|}
\hline Cycle & max speed & avg speed & \% idle & avg spd w/o idle & avg/max spd w/o idle \\
\hline & $\mathrm{mph}$ & $\mathrm{mph}$ & & $\mathrm{mph}$ & \\
\hline CSHVC & 43.84 & 16.03 & 23.18 & 20.87 & 0.48 \\
\hline Freeway & 60.73 & 33.96 & 10.86 & 38.10 & 0.63 \\
\hline Manhattan & 56.58 & 24.22 & 21.72 & 30.98 & 0.55 \\
\hline Yard & 16.80 & 3.33 & 47.35 & 6.32 & 0.38 \\
\hline Test D & 58 & 18.8 & 33.4 & 28.23 & 0.49 \\
\hline Combined & 60.73 & 18.13 & 27.39 & 24.97 & 0.30 \\
\hline
\end{tabular}

\subsection{Drive Cycle Power Requirements}

Several factors affect the power required to drive a vehicle. These factors include the vehicle weight, engine efficiency, driveline efficiency, aerodynamic drag, rolling resistance, road grade, and accessory loads. 
The forces acting on a moving vehicle include aerodynamic drag, rolling resistance, road grade force, and inertial force.

$$
\Sigma F=F_{i}=F_{w}-F_{D}-F_{r r}-F_{\theta}
$$

The aerodynamic drag on an object is based on the density of the fluid it is traveling in, its velocity, its drag coefficient, and its frontal area. It is the force required to push the vehicle through the air.

$$
F_{D}=1 / 2 \rho V^{2} C_{D} A
$$

Rolling resistance comes from a combination of the weight of the vehicle deforming the shape of the tire, the friction between the tire and the roadway, and air friction across the tire surface. [15]

$$
F_{r r}=\mu m g
$$

The force on a vehicle due to road grade is due to a portion of the vehicle's weight vector being directed against the direction of travel when $\theta$ is positive and with the direction of travel when $\theta$ is negative.

$$
F_{\theta}=m g \sin \theta
$$

From basic statics, any object has an associated inertial force.

$$
F_{i}=m \frac{d V}{d t}
$$

Summing the forces on the vehicle yields,

$$
\Sigma F=m \frac{d V}{d t}=-\left(1 / 2 \rho V^{2} C_{D} A+\mu m g+m g \sin \theta\right)+F_{w}
$$

Since power can be calculated from,

$$
\text { Power }=\text { Force }^{*} \text { Velocity }
$$


Multiplying Equation 6 by the vehicle velocity yields,

$$
m V \frac{d V}{d t}=F_{w} V-\left(1 / 2 \rho V^{3} C_{D} A+\mu m g V+m g V \sin \theta\right)
$$

Finally, the power to move an vehicle is based on its aerodynamics, the rolling resistance of its tires, the road grade, and the desired acceleration.

$$
P_{w}=m V \frac{d V}{d t}-\left(1 / 2 \rho V^{3} C_{D} A+\mu m g V+m g V \sin \theta\right)
$$

In addition to the power requirements for driving the vehicle, auxiliary loads and driveline efficiencies can make a significant difference in the power required from the engine. Here, the driveline is assumed to be $95 \%$ efficient although transmissions and differentials tend to become less efficient under low loads. Accessory loads include the power needed to drive air conditioning systems, power steering, and electrical loads. Accessory loads were assumed to be $5 \mathrm{~kW}, 10 \mathrm{~kW}$, and $15 \mathrm{~kW}$ for the Class $2 \mathrm{~B}$, Class 6 , and Class 8 vehicles, respectively. Accessory loads include air conditioning, power steering, cooling fans, alternator, and air compressors. The Class $815 \mathrm{~kW}$ load represents the load from the air conditioner and other accessories for a city bus with a full load of passengers on a hot day. Auxiliary loads for the other classes were scaled according to vehicle weight. This results in an overall power required from the engine.

$$
P_{\text {req engine }}=\frac{P_{\text {req wheels }}}{\eta_{\text {driveline }}}+P_{\text {auxiliary loads }}
$$

The significance of each of the power requirements to drive the vehicle changes under different conditions. When high accelerations are demanded, the inertial term dominates the power requirements. At low speeds, rolling resistance is quite significant compared to aerodynamic drag, but at high speeds, the $\mathrm{V}^{3}$ term causes aerodynamic drag 
to dominate. As the road grade becomes increasingly positive or negative, the resulting power demand or available regenerative braking energy can become quite significant.

In Figures 3.3.1 through 3.3.18, the total energy requirements for the vehicle over the cycle can be found by finding the area between the positive sections of the trace and the $\mathrm{x}$-axis and subtracting the area between the negative sections and the $\mathrm{x}$-axis. The negative portion of the power trace represents the opportunity to capture regenerative braking energy. In a conventional vehicle, this energy is lost through conventional braking.

Figures 3.3.1 through 3.3.3 show the power requirements for each vehicle over the Freeway cycle. The power demand for each vehicle is positive over extended periods of time. There is an obvious lack of opportunity for regenerative braking. In a constant speed situation such as an OTR tractor in the mid-west, even less regenerative braking energy is available and any hybrid system that relies heavily on battery power would be at a major disadvantage. This disadvantage would come from the "dead weight" of the batteries and motor. Since there is little opportunity for regenerative braking, any use of the motor would deplete the batteries without a means to recharge them. This limits the use of the hybrid system and any gains that it might bring and only serves to limit the maximum payload of the truck. The $66 \mathrm{~kW}$ maximum power requirement for the Class 2B vehicle is well below the $163 \mathrm{~kW}$ available power of the average vehicle. While this suggests that the engine is oversized and considerable efficiency improvements might be gained by downsizing the powerplant, consumer demand for high performance from this class limits the extent of change that would still meet consumer acceptability. The power requirements of the Class 6 vehicle exceed the $125 \mathrm{~kW}$ ceiling during a few peak 
accelerations, but the end effect on performance over the cycle would be minimal and well within acceptable performance bounds. Similarly, the Class 8 power trace exceeds the $205 \mathrm{~kW}$ limit over several periods of the cycle, but, again, consumer expectations of dynamic performance are quite low. Also, the power needed to exactly meet the trace would require a very powerful engine or engine-electric motor combination that would tend to be oversized and inefficient under less demanding situations.

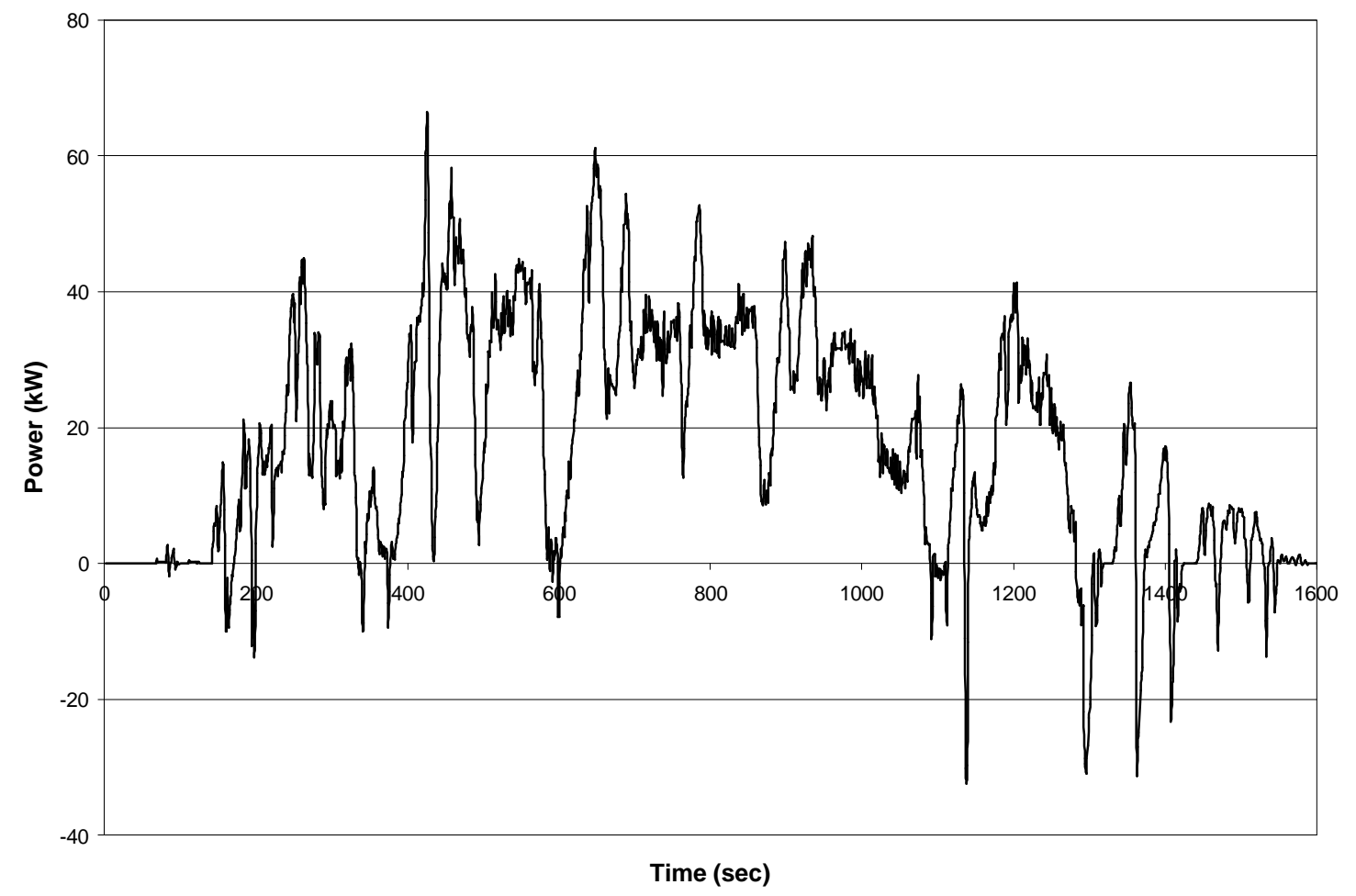

Figure 3.3.1 Power required for Class 2B vehicle over the Freeway Cycle. 


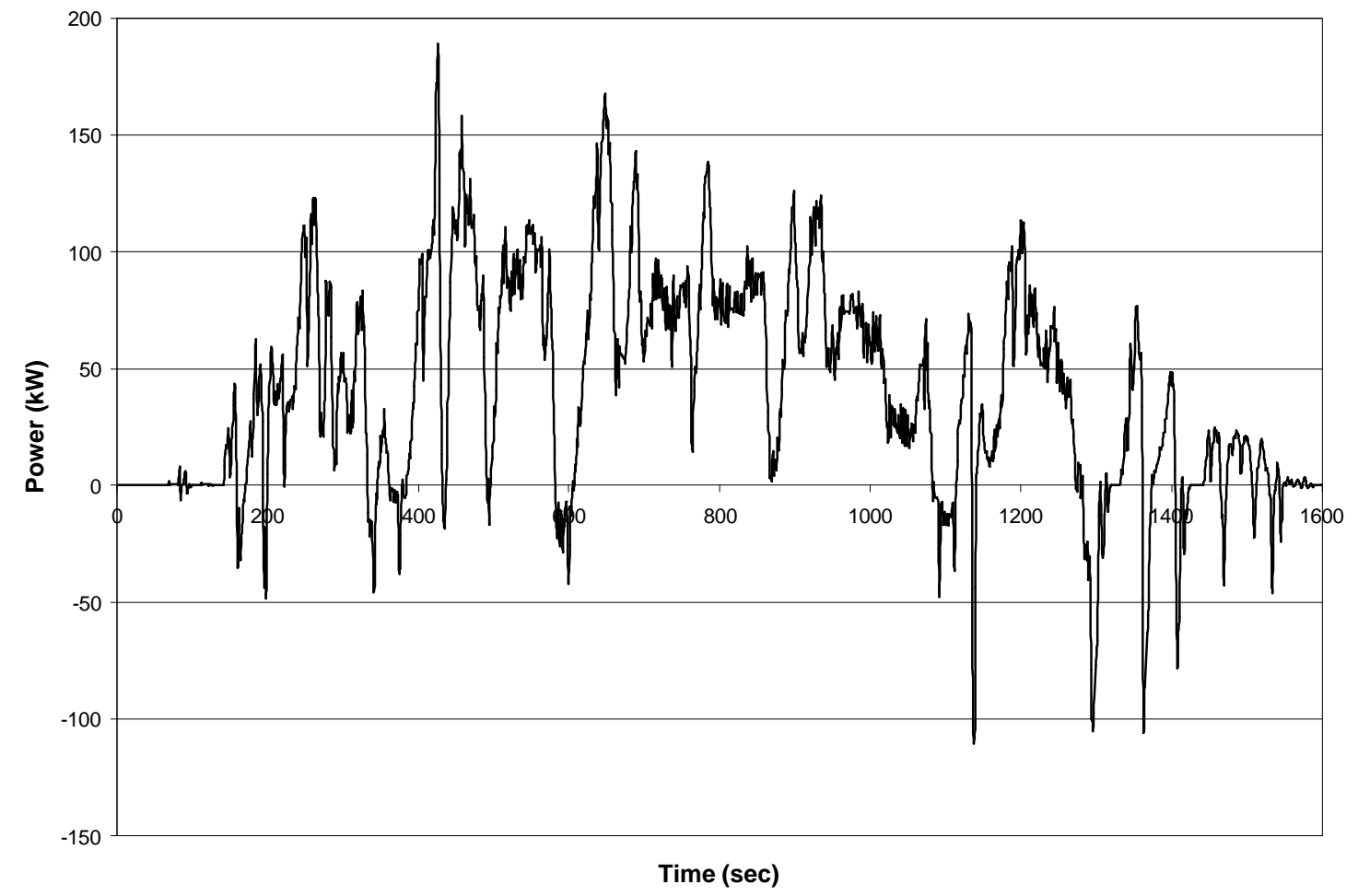

Figure 3.3.2 Power required for Class 6 vehicle over the Freeway Cycle.

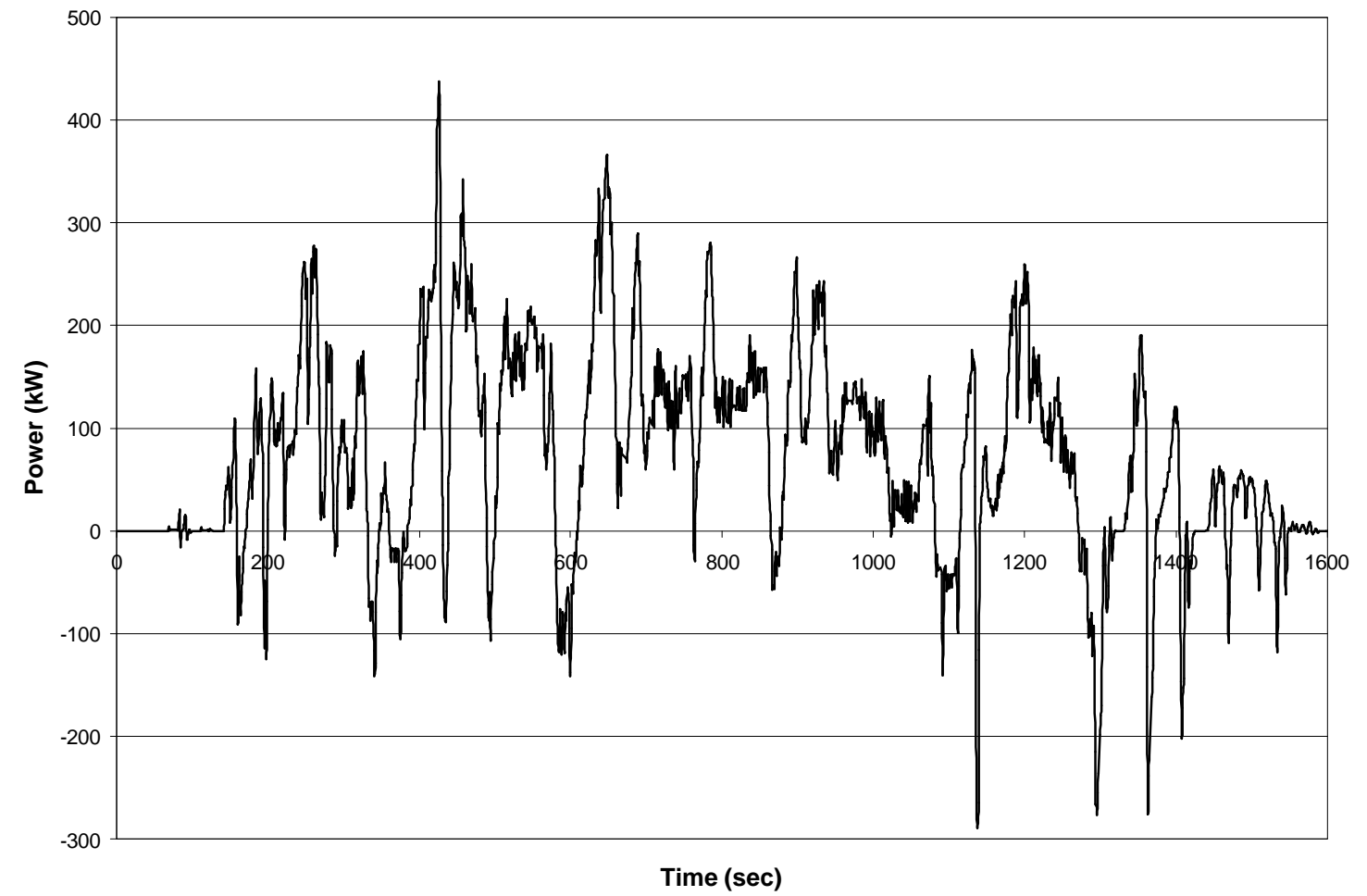

Figure 3.3.3 Power required for Class 8 vehicle over the Freeway Cycle. 
Figures 3.3.4 through 3.3.6 show the power requirements for each vehicle over the CSHVC. This cycle is much more transient than the Freeway Cycle. While there are several large power spikes, there is a large amount of regenerative braking energy available. Peak power requirement only exceeds the power available to the vehicle for Class 8.

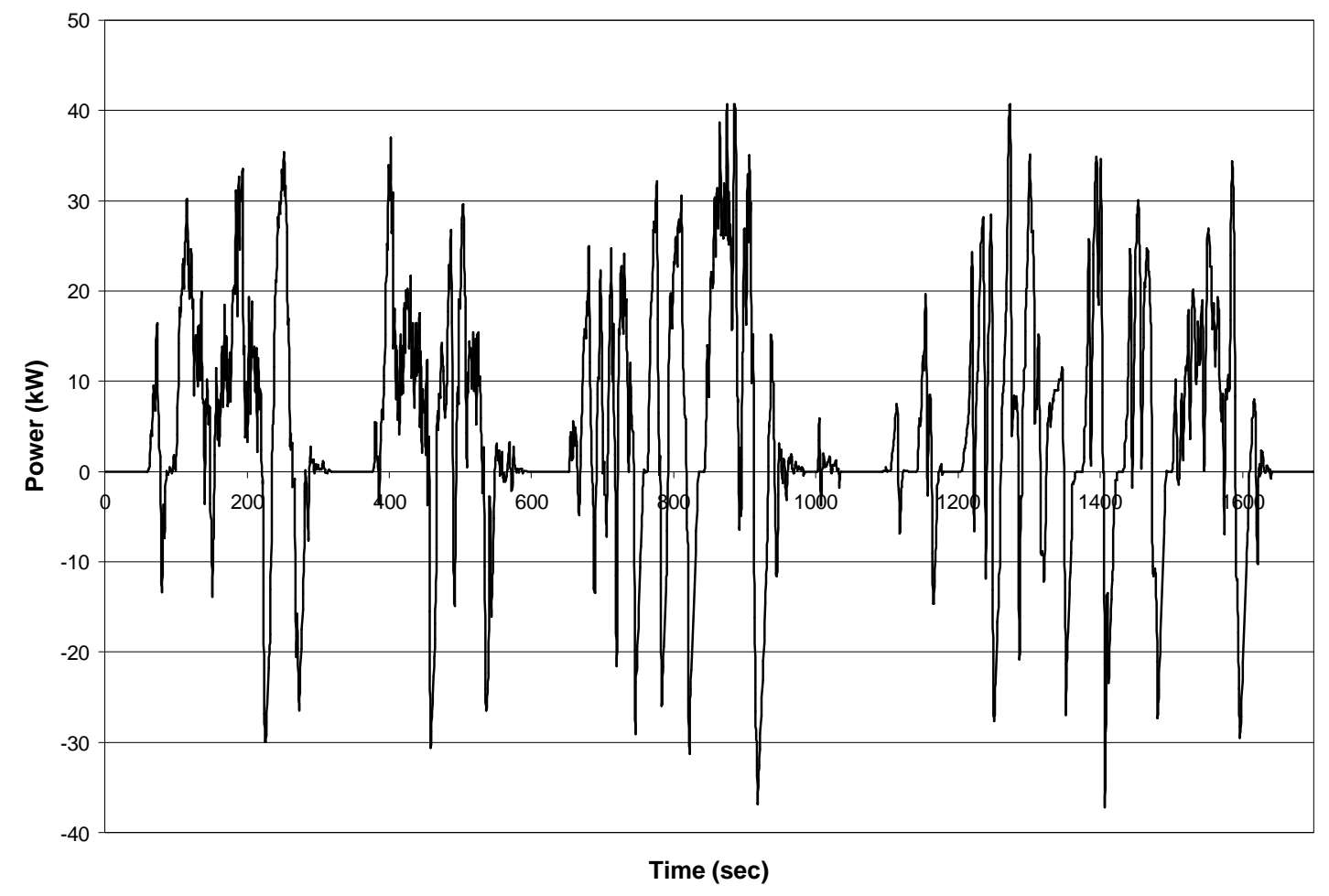

Figure 3.3.4 Power required for Class 2B vehicle over the CSHVC. 


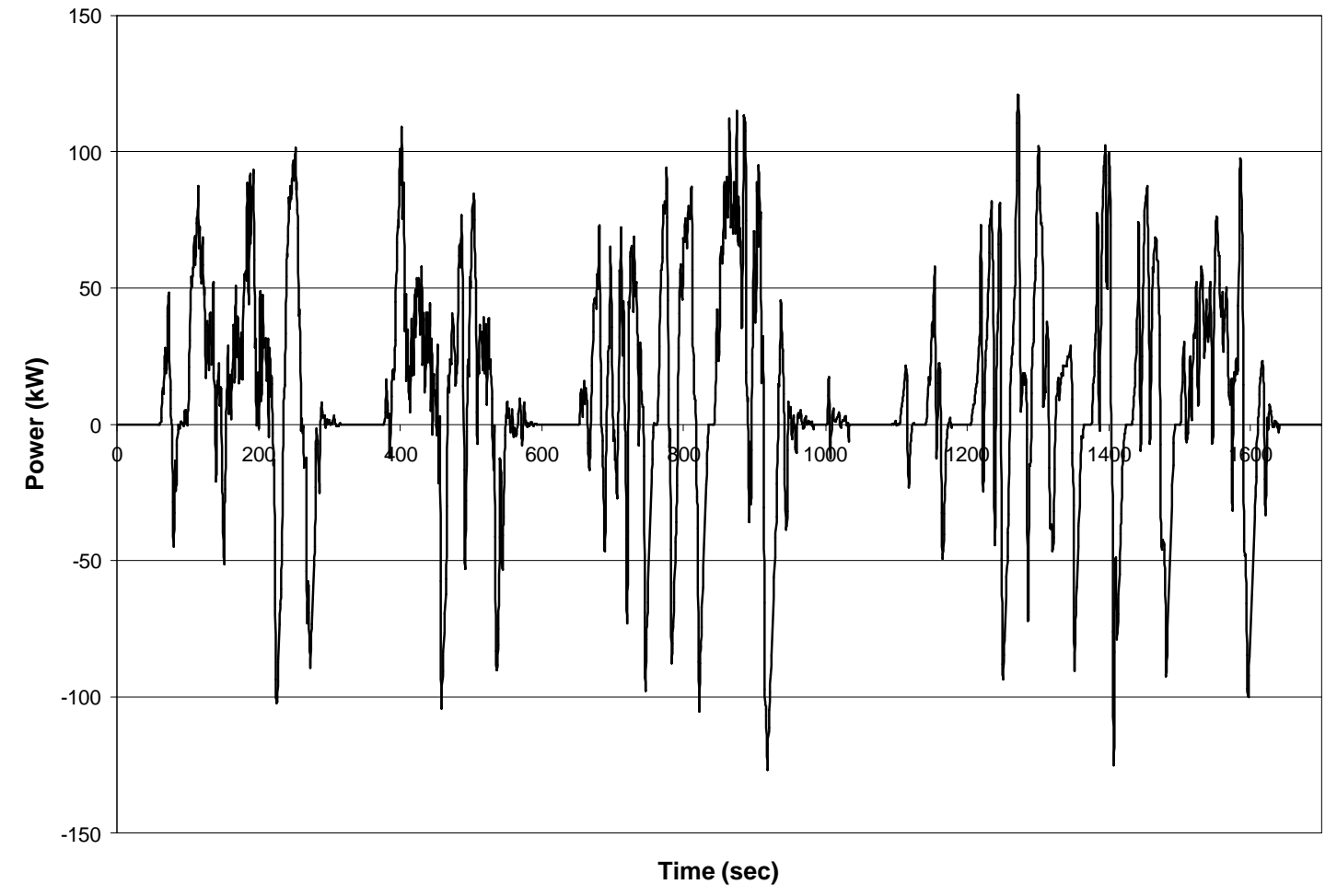

Figure 3.3.5 Power required for Class 6 vehicle over the CSHVC.

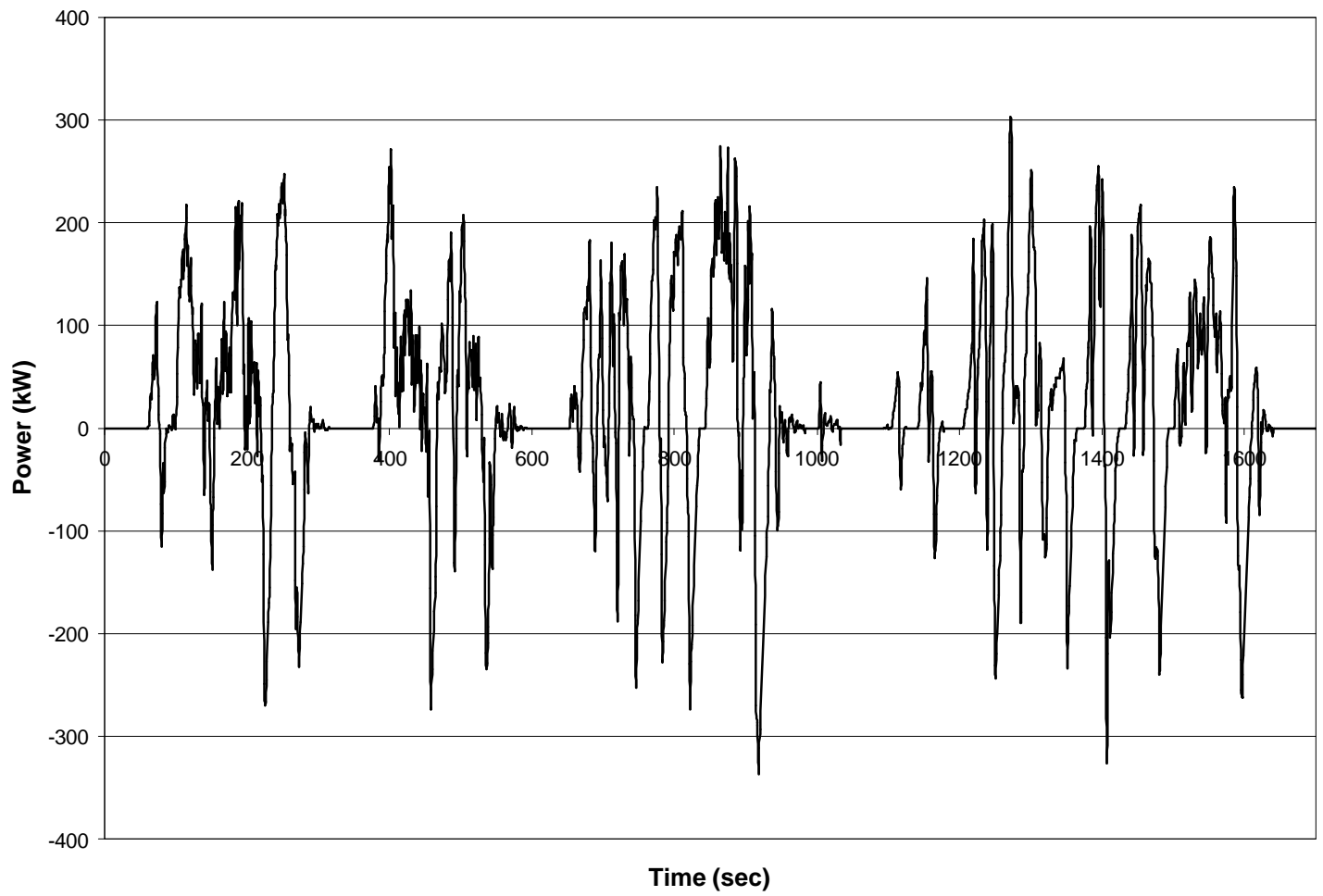

Figure 3.3.6 Power required for Class 8 vehicle over the CSHVC. 
Figures 3.3.7 through 3.3.9 show the power requirements for each vehicle over the Yard Cycle. The low speeds and accelerations in this cycle result in extremely low power requirements. This, combined with fairly significant available regenerative braking energy, results in average power requirements close to zero. The Class 2B vehicle requires only $14 \mathrm{~kW}$ of peak power to meet the cycle while the Class 6 and 8 vehicles require 40 and $105 \mathrm{~kW}$ respectively.

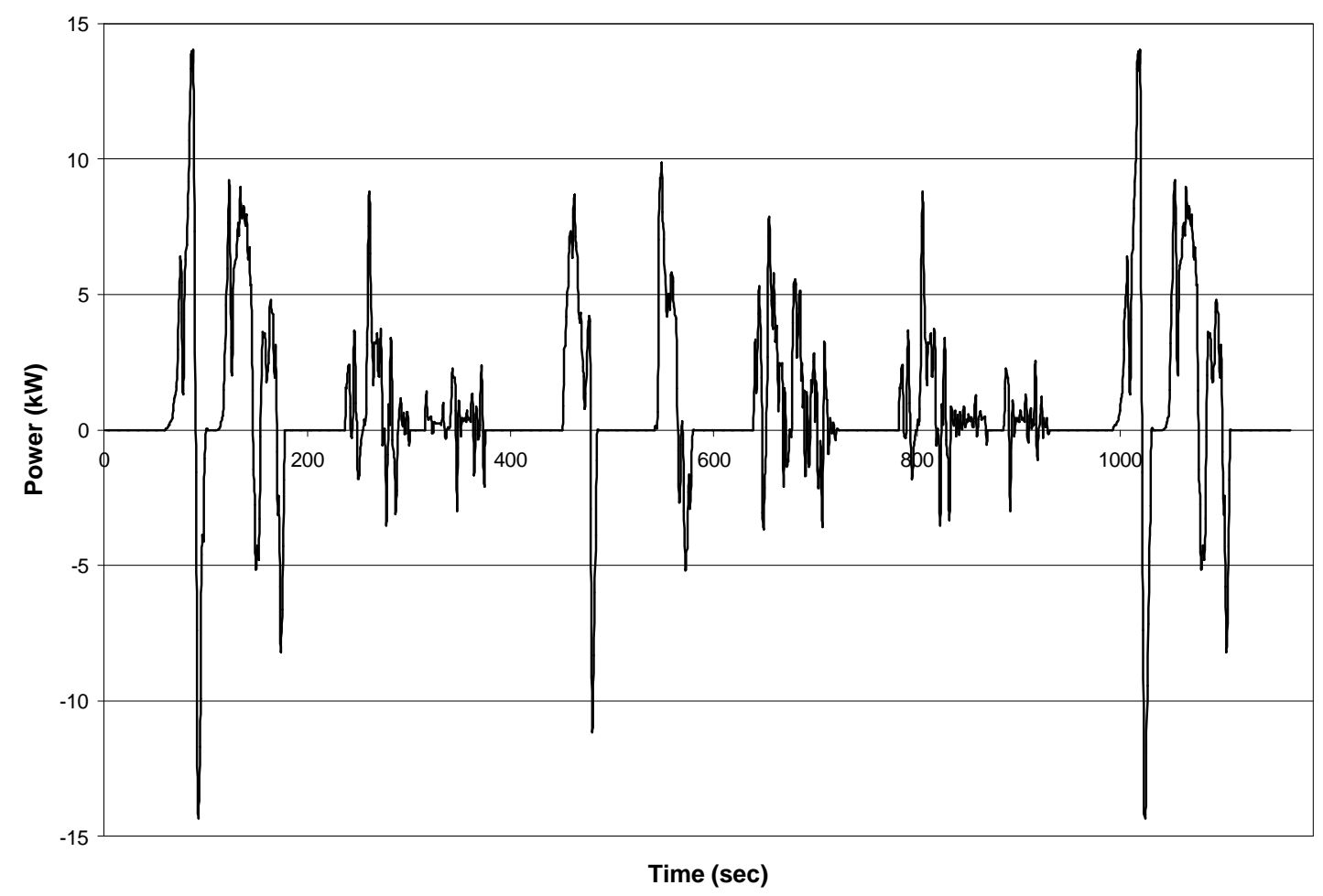

Figure 3.3.7 Power required for Class 2B vehicle over the Yard Cycle. 


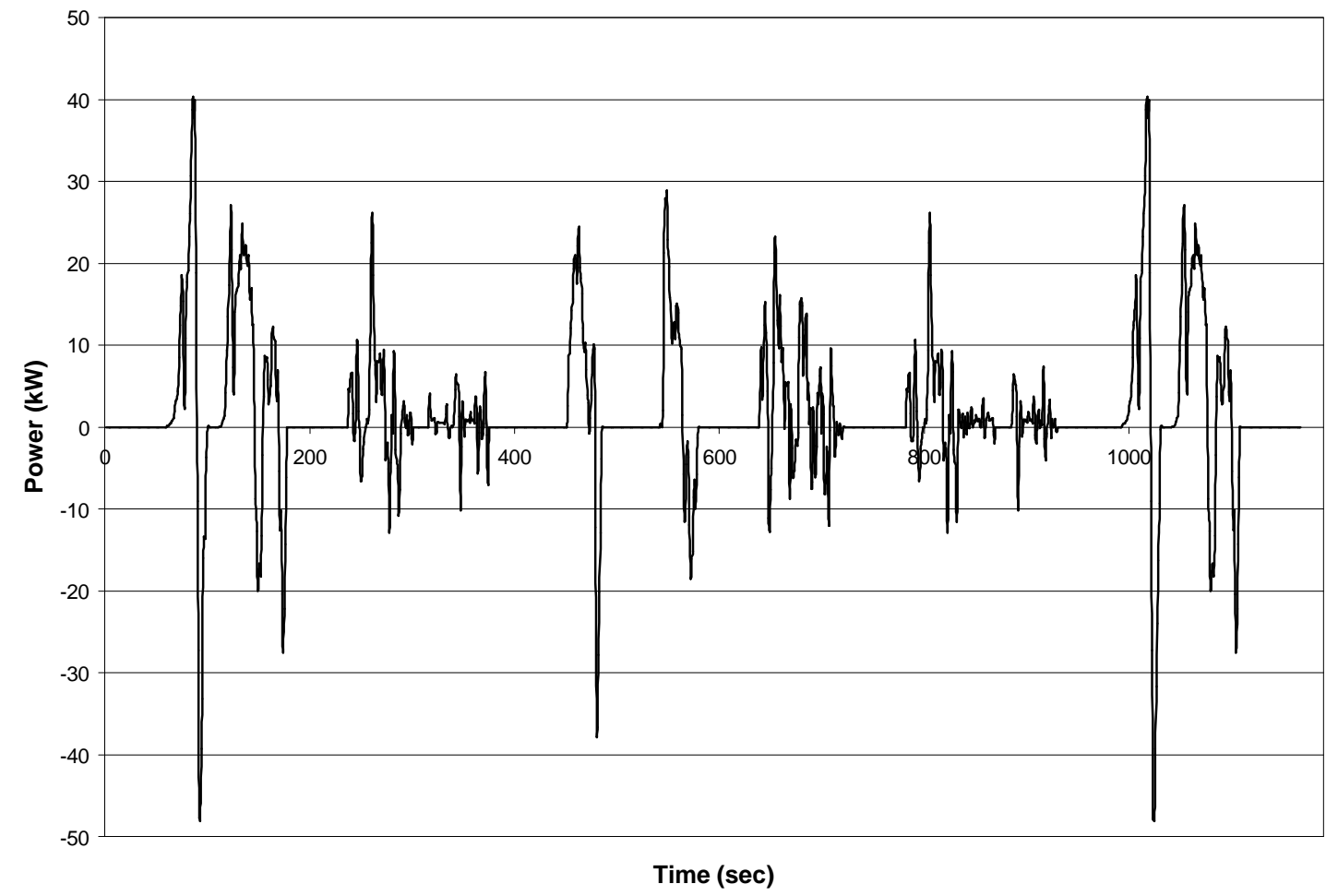

Figure 3.3.8 Power required for Class 6 vehicle over the Yard Cycle.

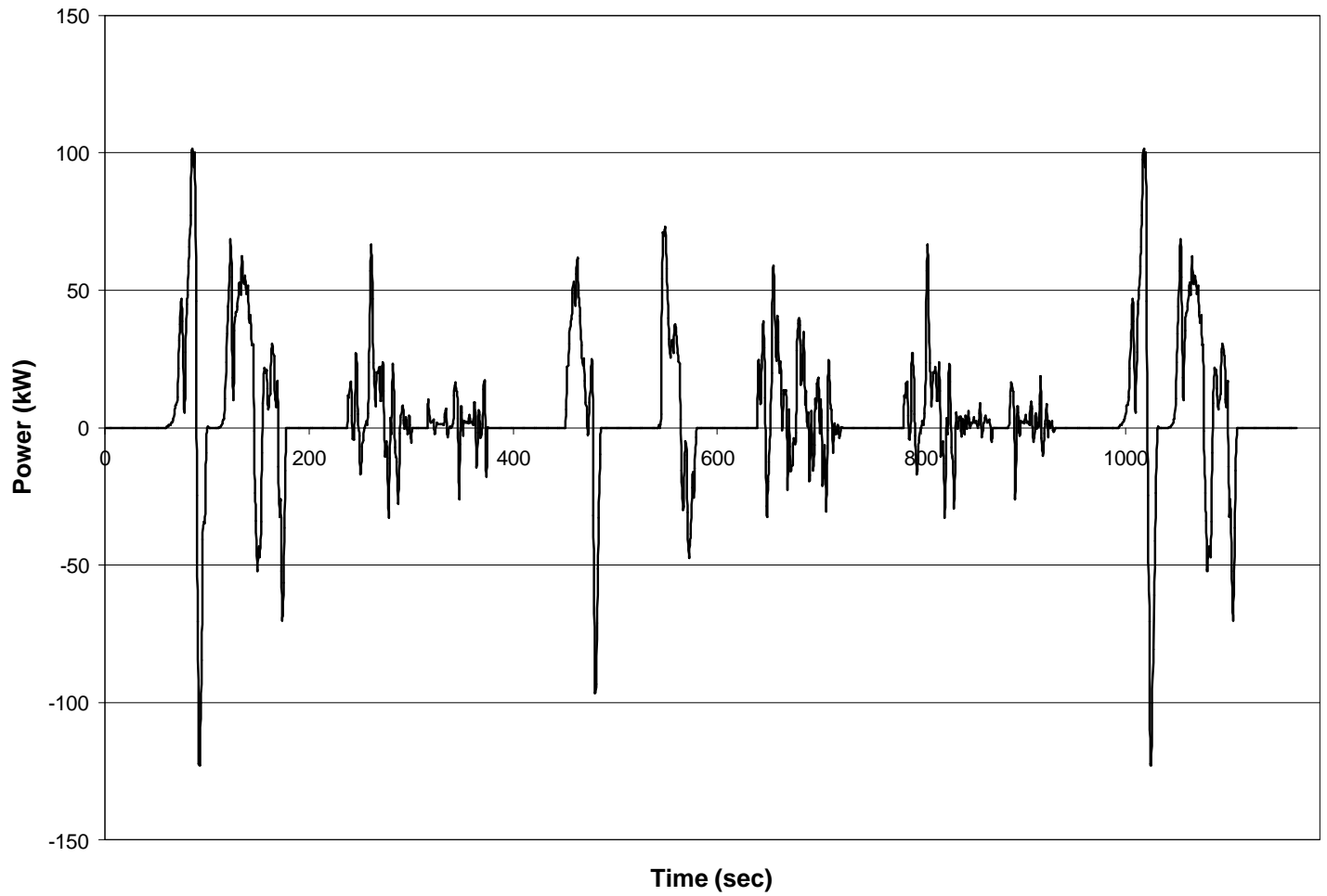

Figure 3.3.9 Power required for Class 8 vehicle over the Yard Cycle. 
Figures 3.3.10 through 3.3.12 show the power requirements for each vehicle over the Manhattan Cycle. This cycle is very repetitive in that it follows a pattern of quite similar accelerations and decelerations. This results in a very low average power requirement. While power spikes for the Class 6 and 8 vehicles are over the limits, they are not excessive. The limited power available to the average vehicles would not result in large deviations from the trace.

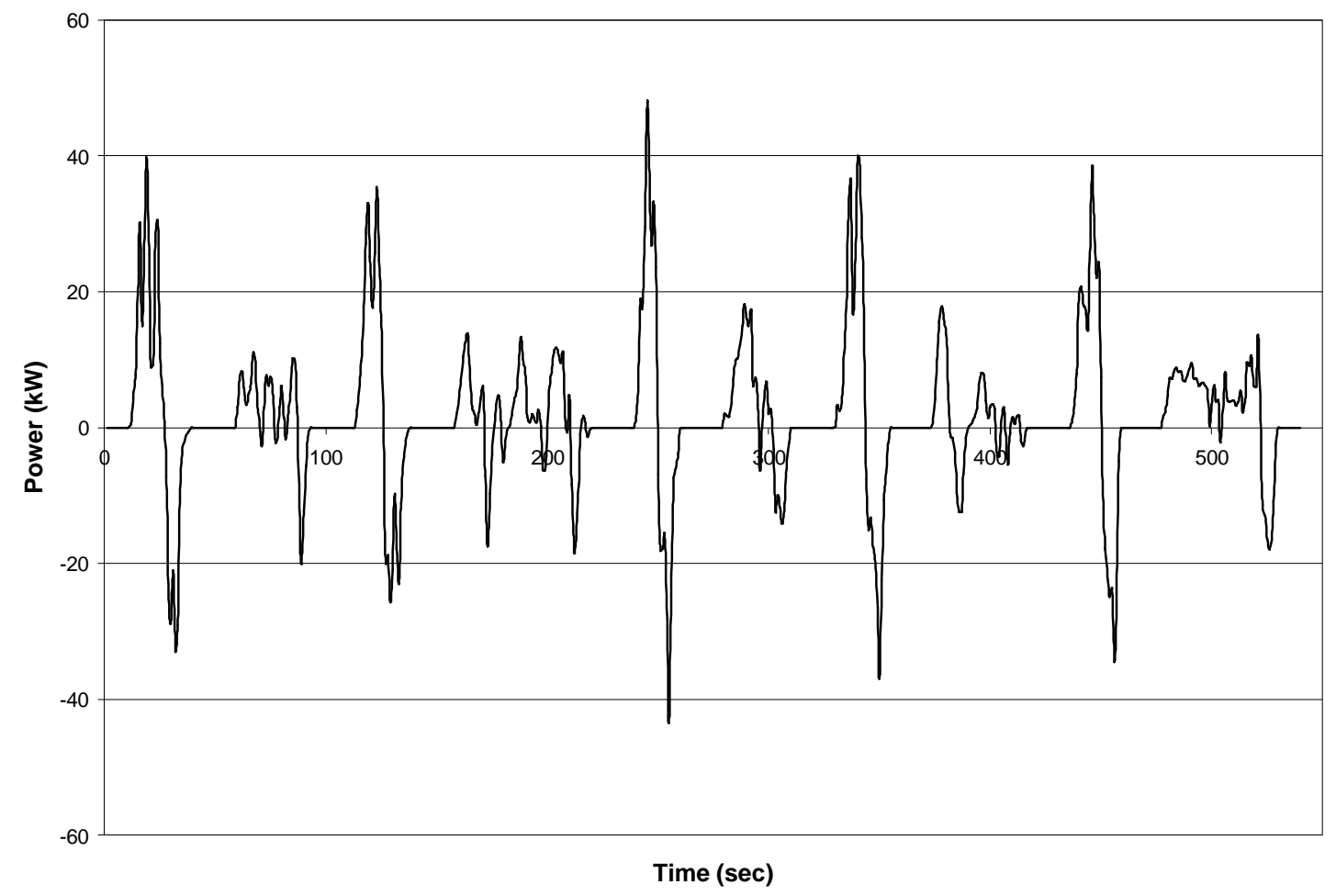

Figure 3.3.10 Power required for Class 2B vehicle over the Manhattan Cycle. 


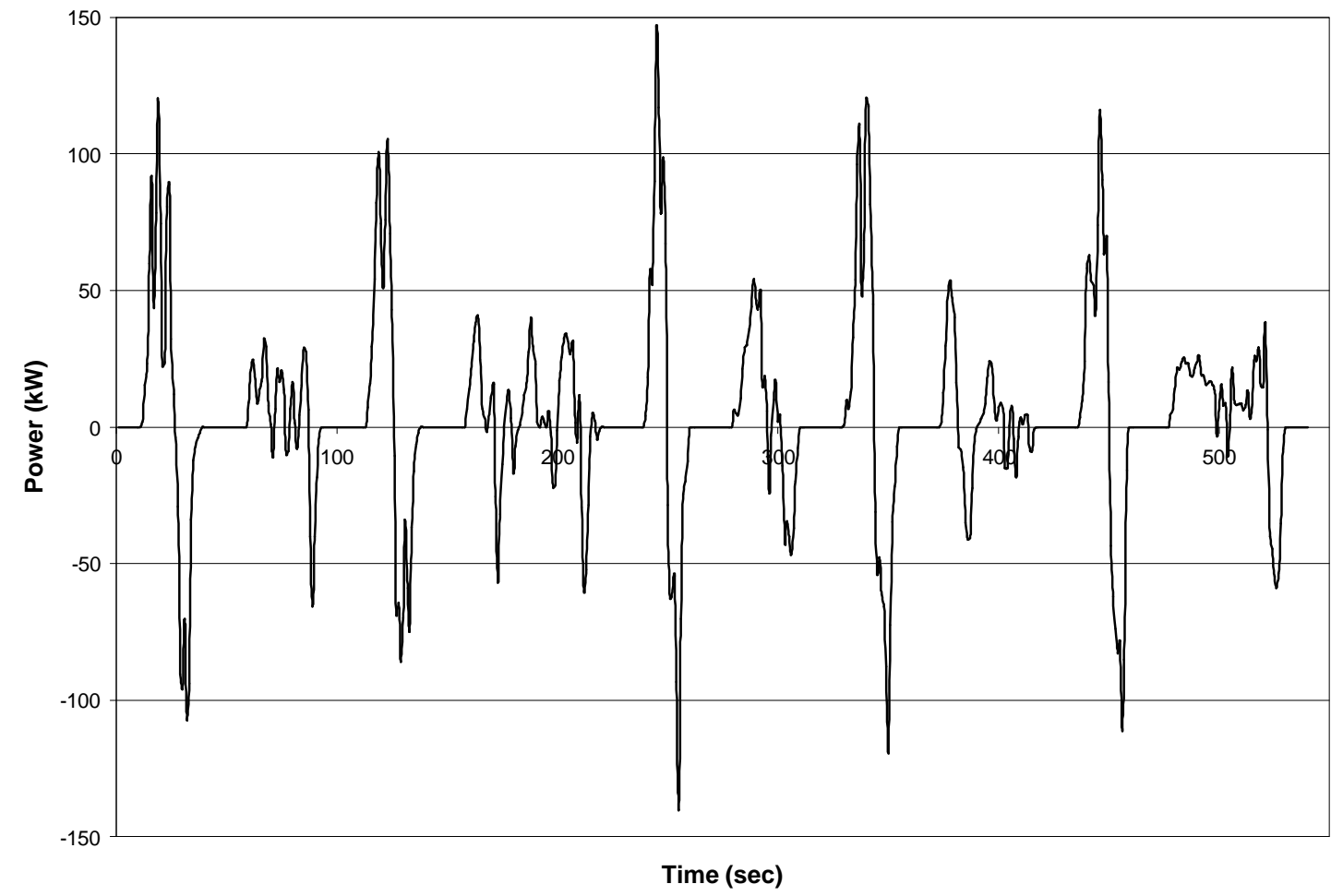

Figure 3.3.11 Power required for Class 6 vehicle over the Manhattan Cycle.

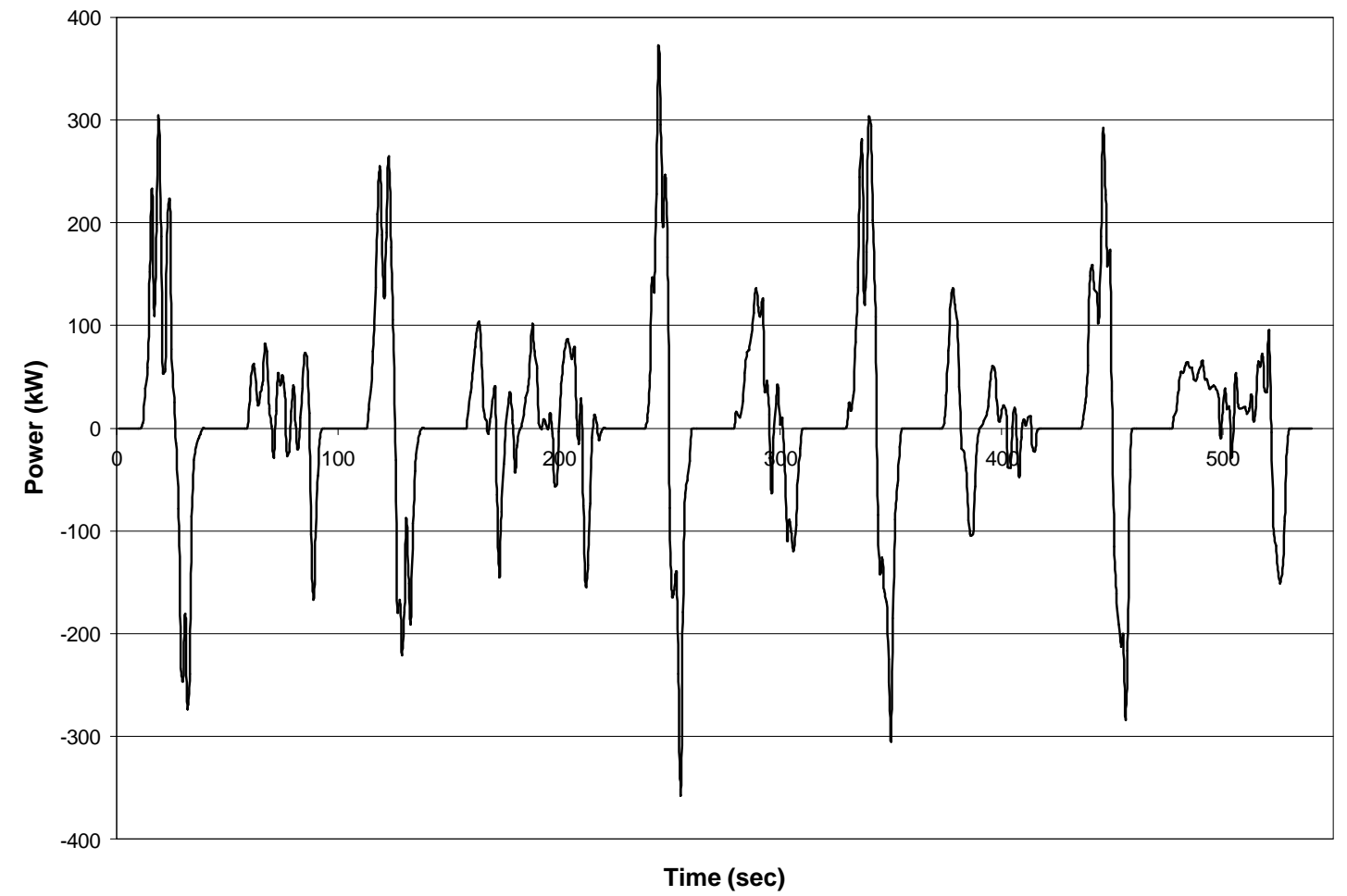

Figure 3.3.12 Power required for Class 8 vehicle over the Manhattan Cycle. 
Figures 3.3.13 through 3.3.15 show the power requirements for each vehicle over the Test D Cycle.

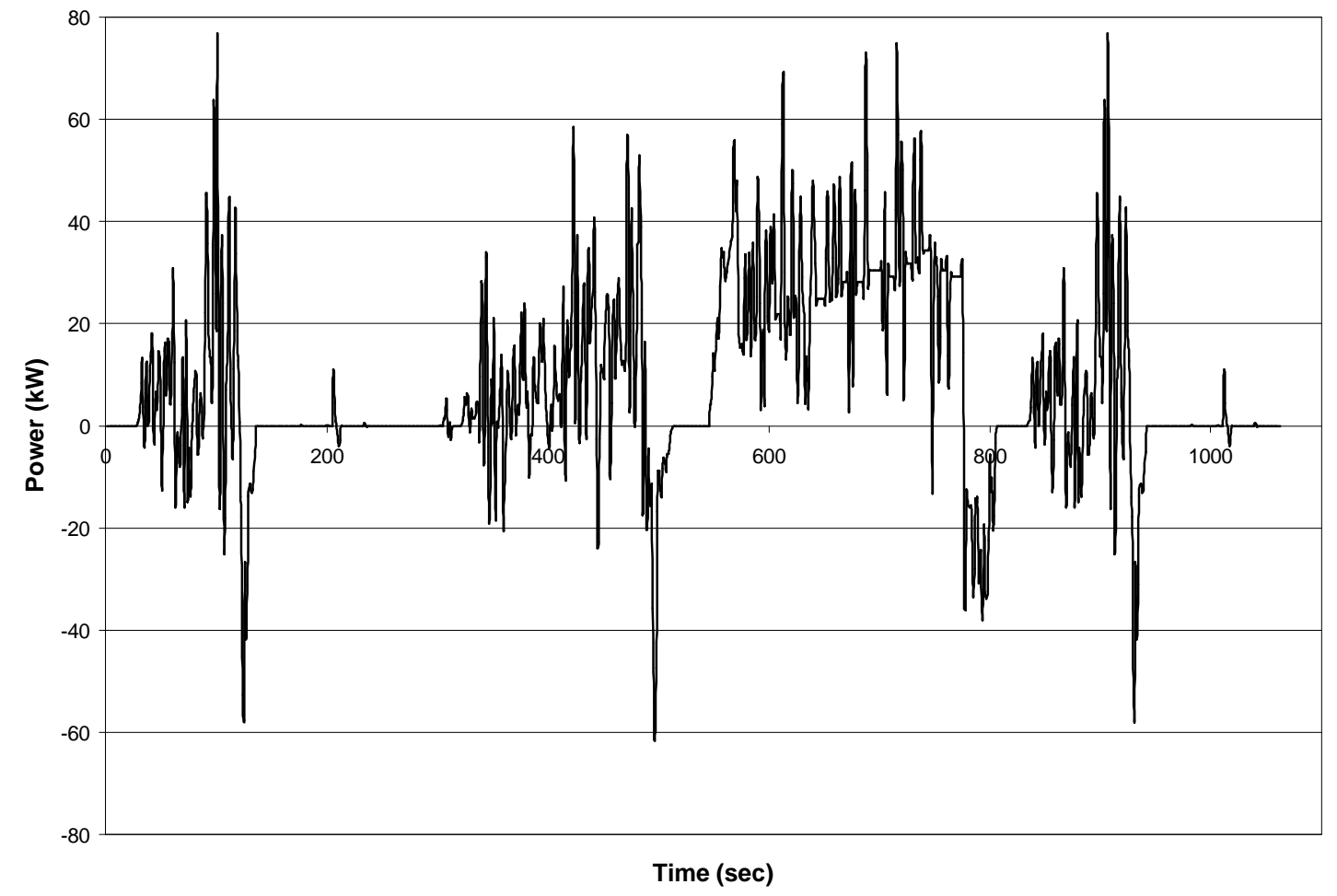

Figure 3.3.13 Power required for Class 2B vehicle over the Test D Cycle. 


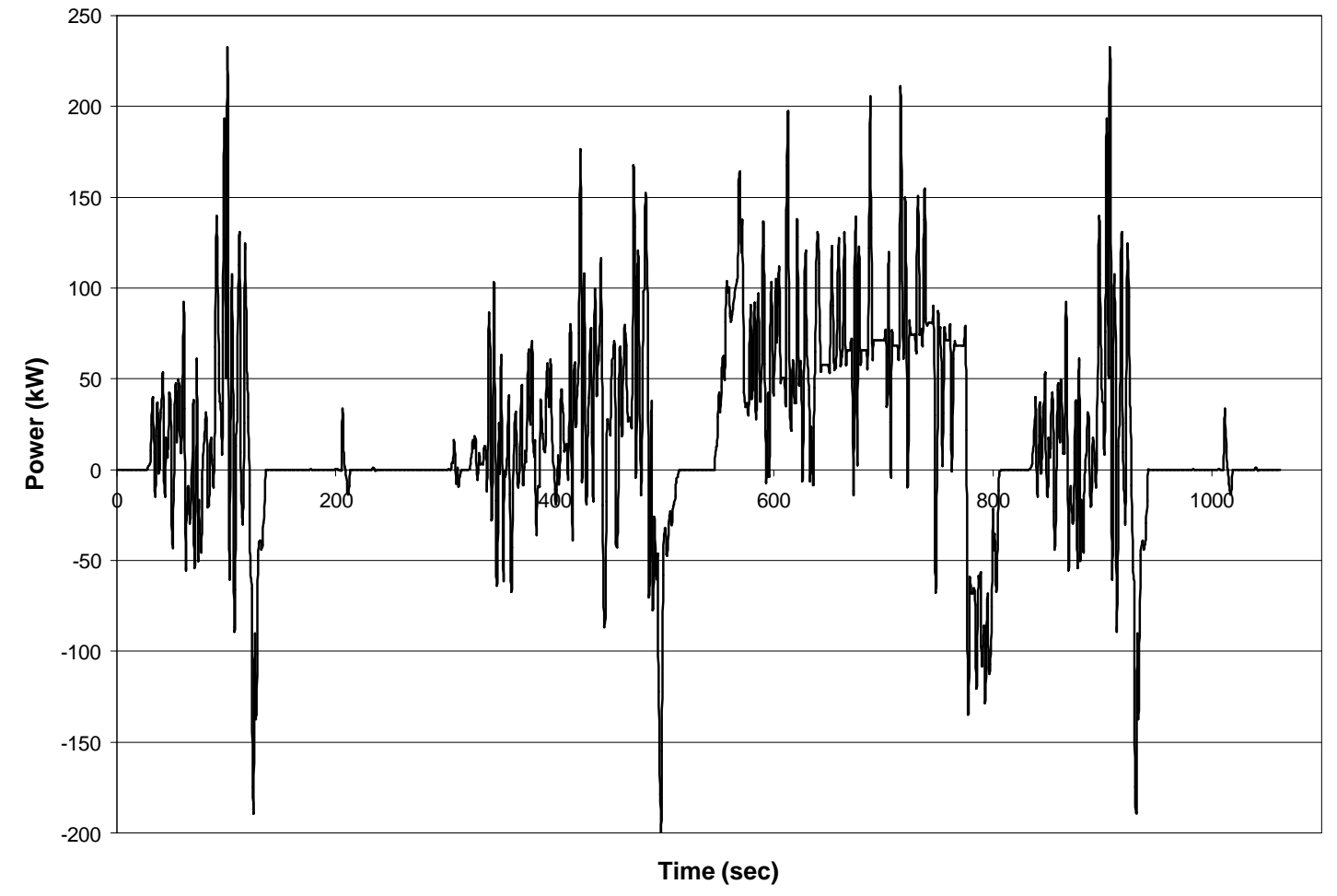

Figure 3.3.14 Power required for Class 6 vehicle over the Test D Cycle.

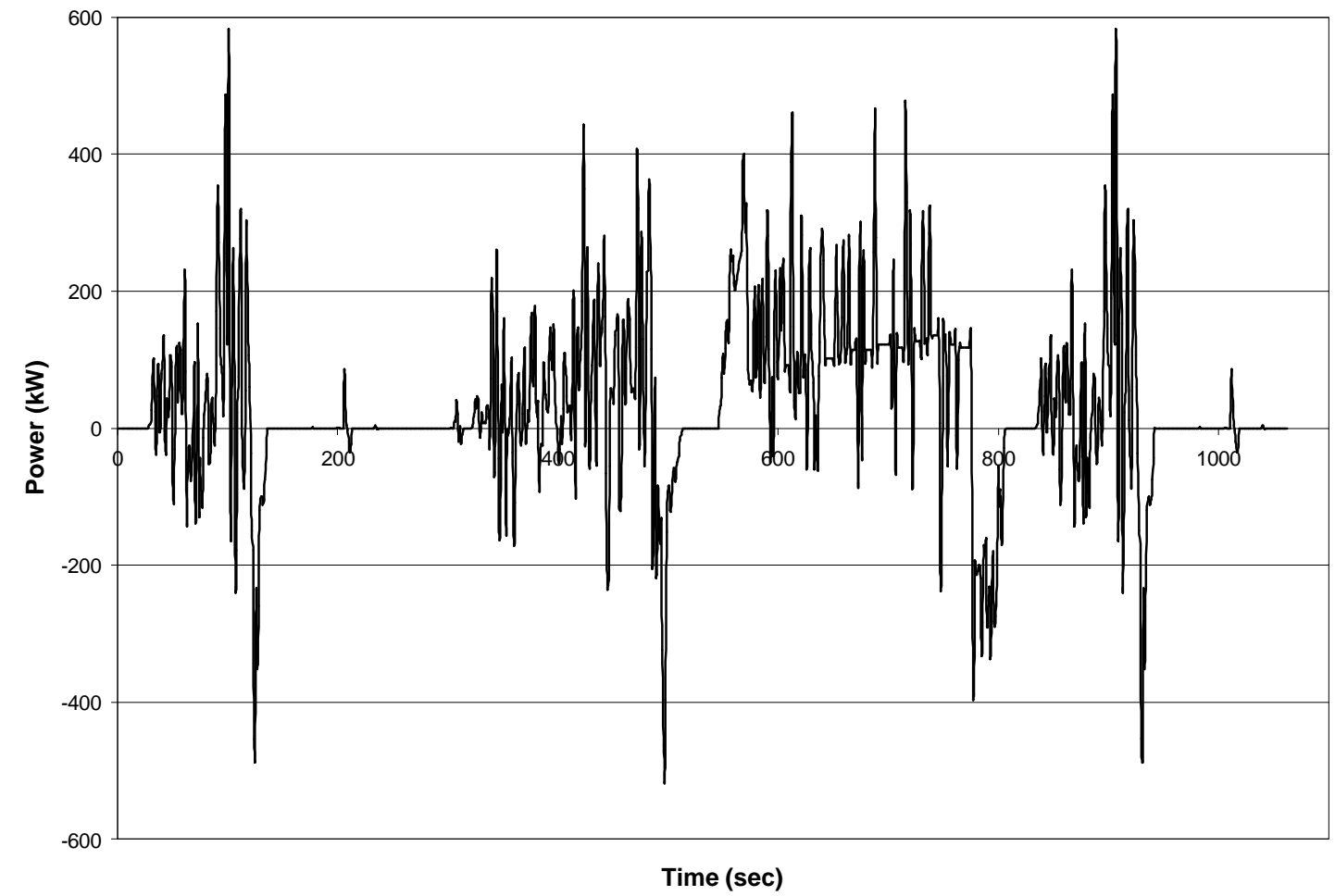

Figure 3.3.15 Power required for Class 8 vehicle over the Test D Cycle. 
Figures 3.3.16 through 3.3.18 show the power requirements for each vehicle over the Combined Cycle.

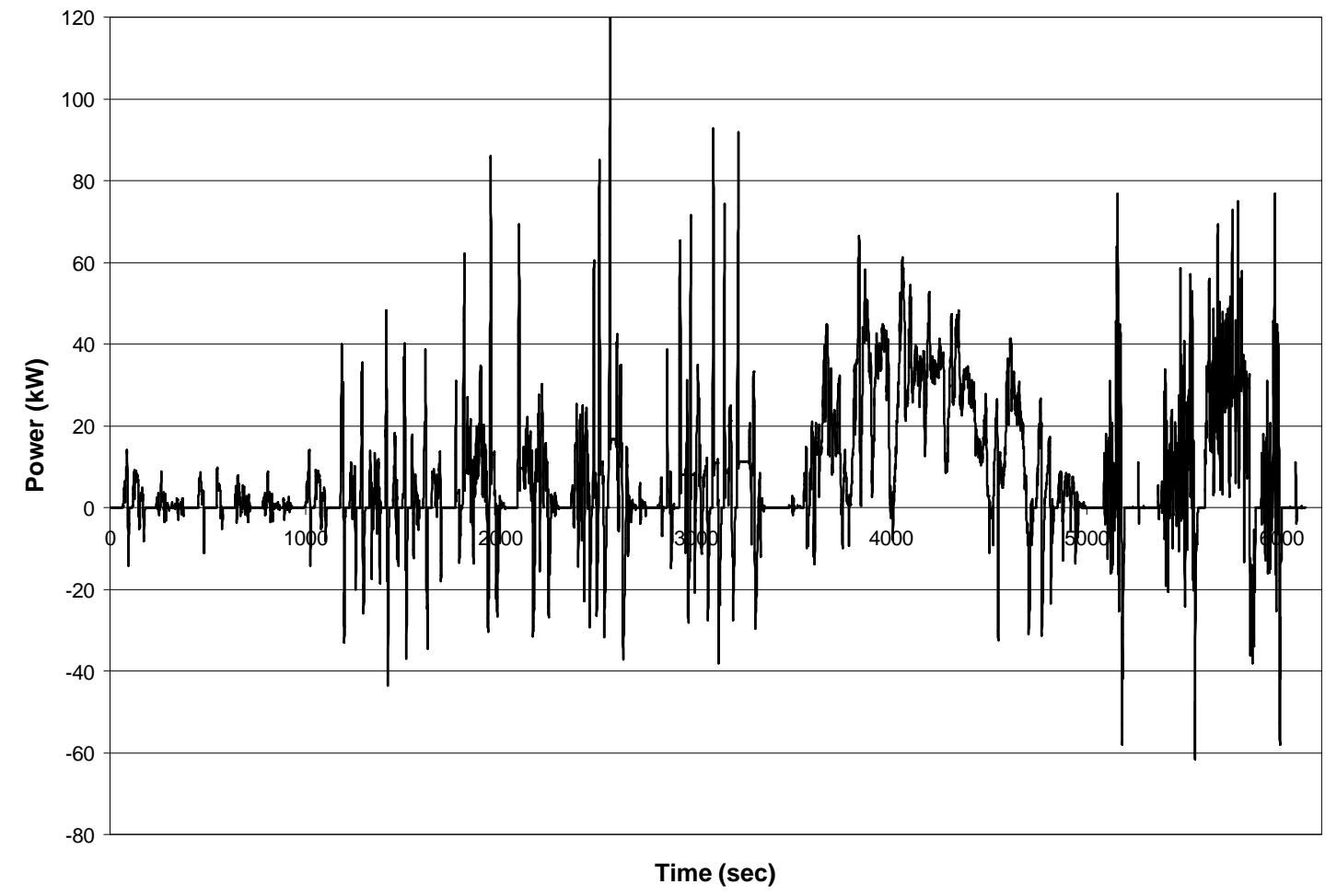

Figure 3.3.16 Power required for Class 2B vehicle over the Combined Cycle. 


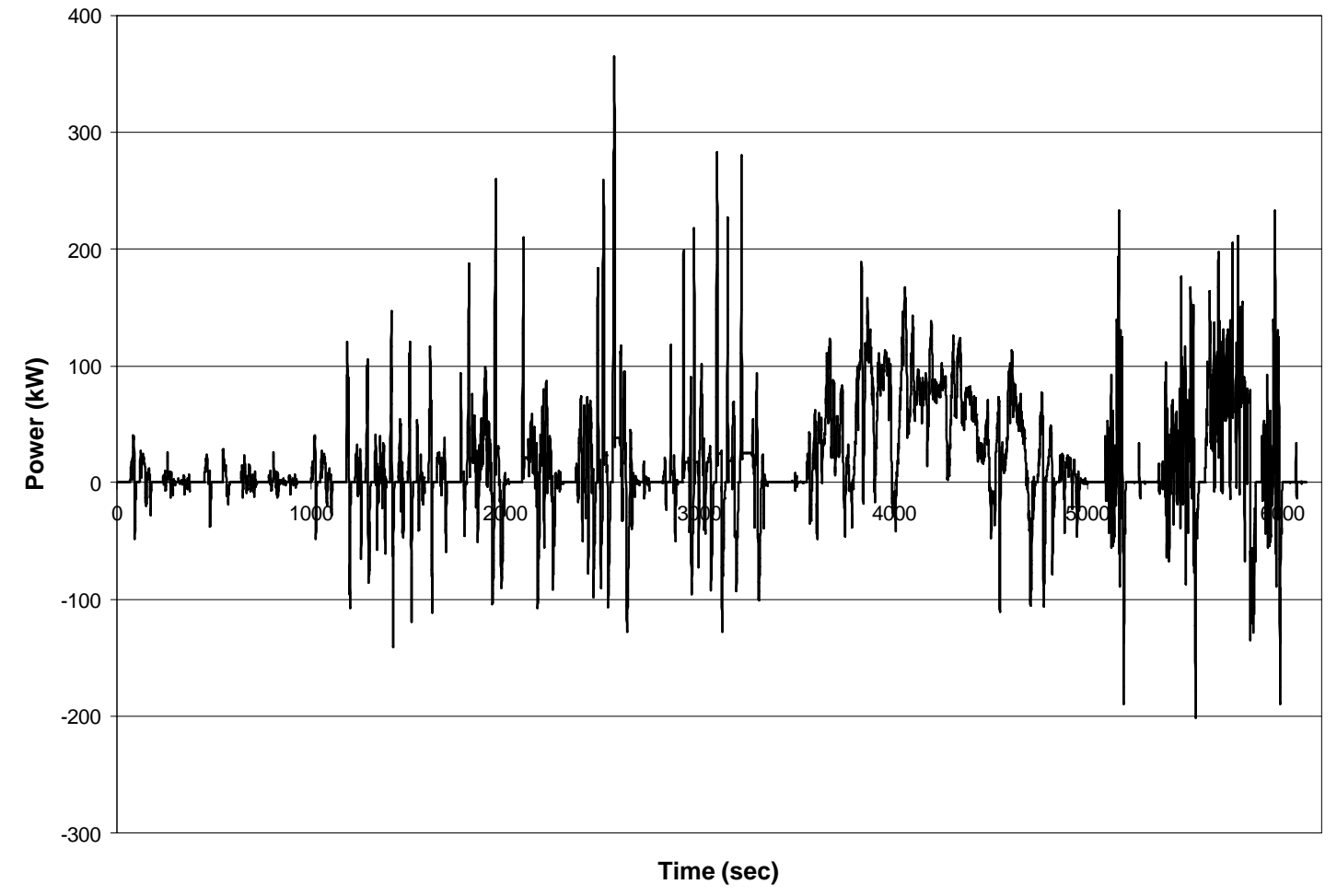

Figure 3.3.17 Power required for Class 6 vehicle over the Combined Cycle.

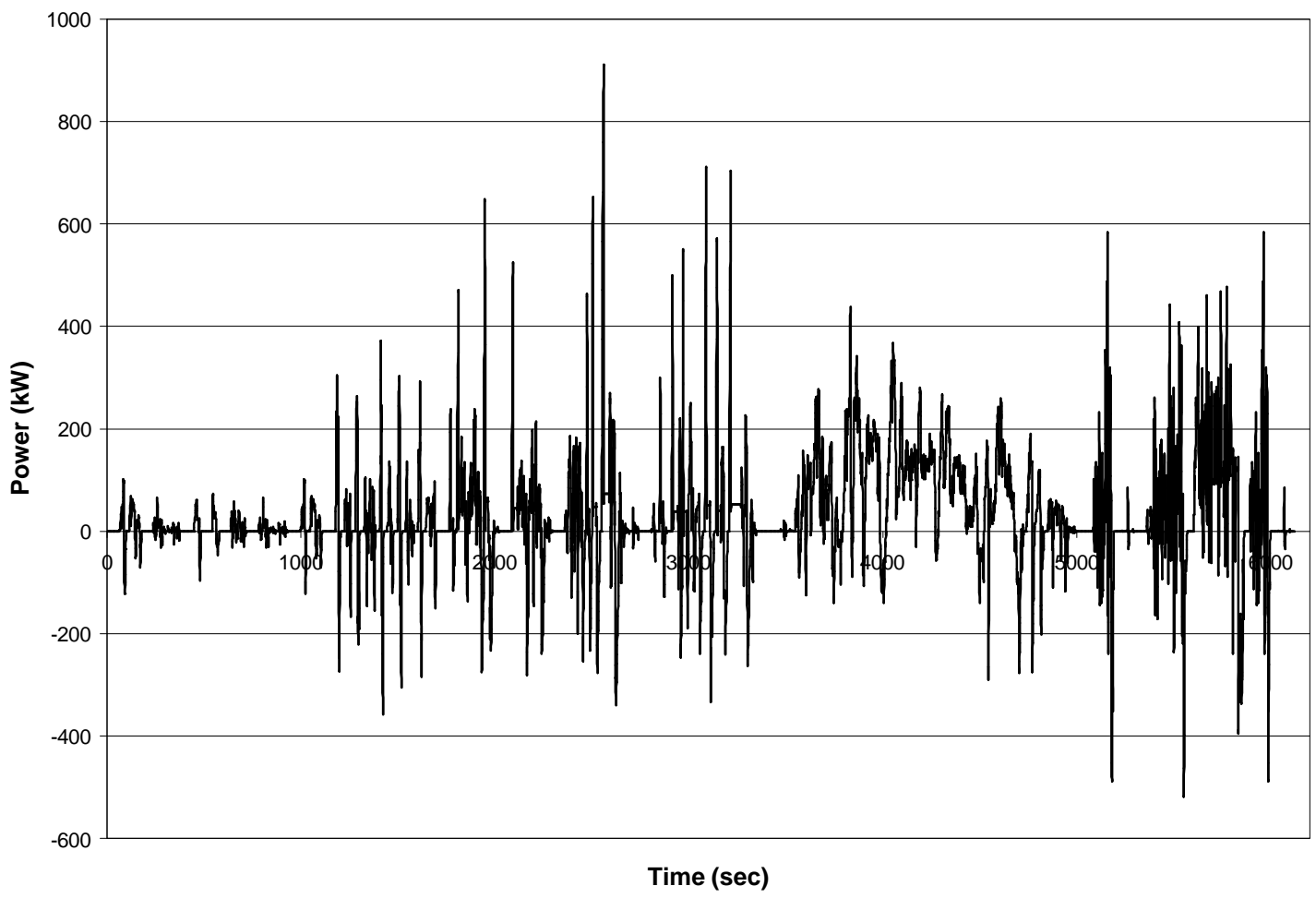

Figure 3.3.18 Power required for Class 8 vehicle over the Combined Cycle. 


\subsection{Simulation}

Given an instantaneous power required at the vehicle wheels determined from the road load equation, the power requirements from the ICE, electric motor, and batteries can be determined. Similar control strategies were used to simulate the series and parallel HEVs.

\subsubsection{Series Control Strategy}

In the series HEV control strategy, the ICE is run at a constant percentage of the road load power, $\mathrm{C}_{1}$, plus or minus a State of Charge $(\mathrm{SoC})$ correction factor, as shown in Equation 11 while the electric motor supplies or absorbs the balance of the power required at the wheels.

$$
P_{e}=C_{1} P_{w}-C_{2}\left(S o C_{i}-S o C_{t}\right)
$$

The SoC correction factor demands more power from the ICE when the actual SoC falls below the target value and decreases the demand on the ICE when the target SoC is exceeded. This also serves to smooth the power from the ICE relative to the road load power. The power from the ICE is delivered directly to the electric motor through a generator to avoid the losses associated with using the batteries while the electric motor draws power from or delivers power to the batteries. Additionally, the ICE can be set to run at a minimum power to account for any auxiliary loads associated with operating the vehicle.

\subsubsection{Parallel Control Strategy}

The parallel HEV uses the same control strategy equation as the series vehicle, but the power from the engine is directed to the wheels and accounts for a portion of the power demanded by the road load equation. The balance of the road load power is 
provided by the electric motor. As in the series vehicle, a provision is made to account for auxiliary loads.

For the simulation of both the parallel HEV and conventional vehicle, the issue of gear-shifts and turbo lag are avoided. During gear-shifts with a manual transmission, the power delivered from the engine to the wheels briefly drops to zero. During the power drop and during transients from low to high power, turbo lag becomes important. Turbocharged diesel engines such as those used in most automotive applications use exhaust driven turbines to drive compressors to increase the density of the intake air charge allowing for more fueling and power. At low engine speeds, the flow of exhaust gases is too low to properly spool the turbine and no power gains are seen. There is a brief time constant associated with sudden power demands by the driver through the accelerator pedal and speeding the engine to the point that the turbo-charger becomes effective and the engine delivers the power desired. This is also an issue when the engine speed drops during gear-shifts. Analysis of this issue would affect the results of the simulation.

\subsubsection{Battery Model and Simulation}

A simplified battery model was used to simulate the flow of power into and out of the batteries. Hawker Genesis G13EP batteries were chosen due to their availability, proper design and hardware for EV and HEV use, and low internal resistance.

\section{Table 3.4.1 Hawker Genesis G13EP Battery Properties. [16]}

\begin{tabular}{|c|c|}
\hline Product & Hawker Genesis G13EP \\
\hline Battery Type & Lead-Acid \\
\hline Capacity & $13 \mathrm{Ah}$ \\
\hline Nominal Full-charge Voltage & $12.85 \mathrm{~V}$ \\
\hline Internal Resistance & $8.5 \mathrm{~m} \Omega$ \\
\hline Weight & $4.9 \mathrm{~kg}$ \\
\hline
\end{tabular}


The energy capacity of the batteries in joules can be calculated from

$$
E_{b}=3600 V_{b} C_{b} N_{b}
$$

To achieve the 300-400V operating range of the electric motors typically used in EV and HEV operations, 27 batteries were combined in a resulting in a $324 \mathrm{~V}$ nominal voltage pack. Three battery packs were used in the Class 6 and 8 HEVs while one pack was used in the Class $2 \mathrm{~B}$ vehicle.

Once the power required from the electric motor is known from the control strategy, the power demand from the batteries can be calculated. From the SoC of the batteries, a nominal voltage is known based on full charge and an effective empty level.

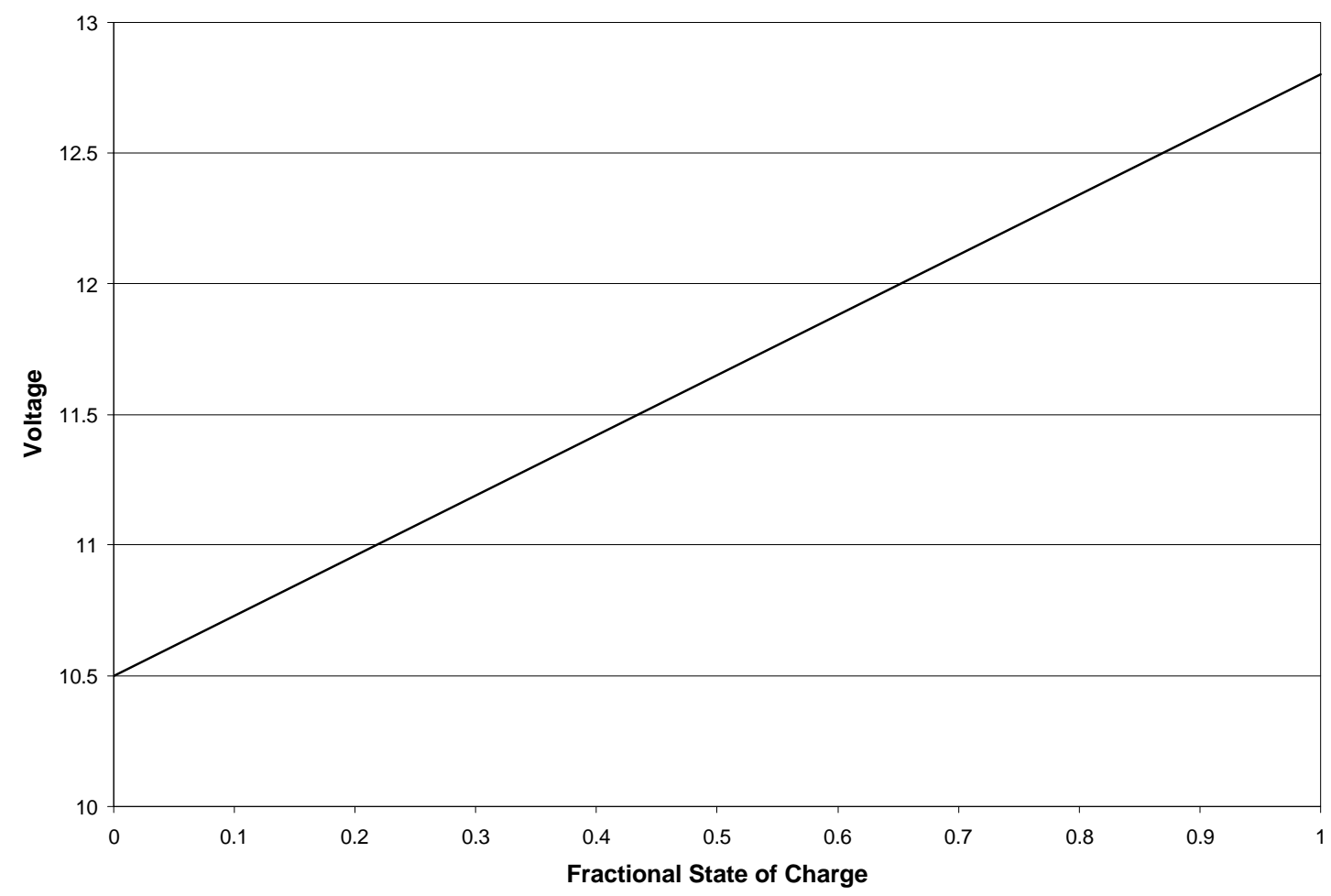

Figure 3.4.1 Battery voltage vs. SoC for Hawker Genesis G13EP. [16]

The SoC of the battery pack at any instant can be calculated from,

$$
S o C_{i}=\frac{3600 V_{b i} C_{b} N_{b}}{E_{b}}
$$


This determines the voltage of the battery pack. From this voltage, a current draw can be calculated based on,

$$
I_{b}=\frac{P_{m}}{V_{b}}
$$

Batteries have internal resistance resulting in power losses during discharge and charging. These losses are approximated based on the current demand on the batteries.

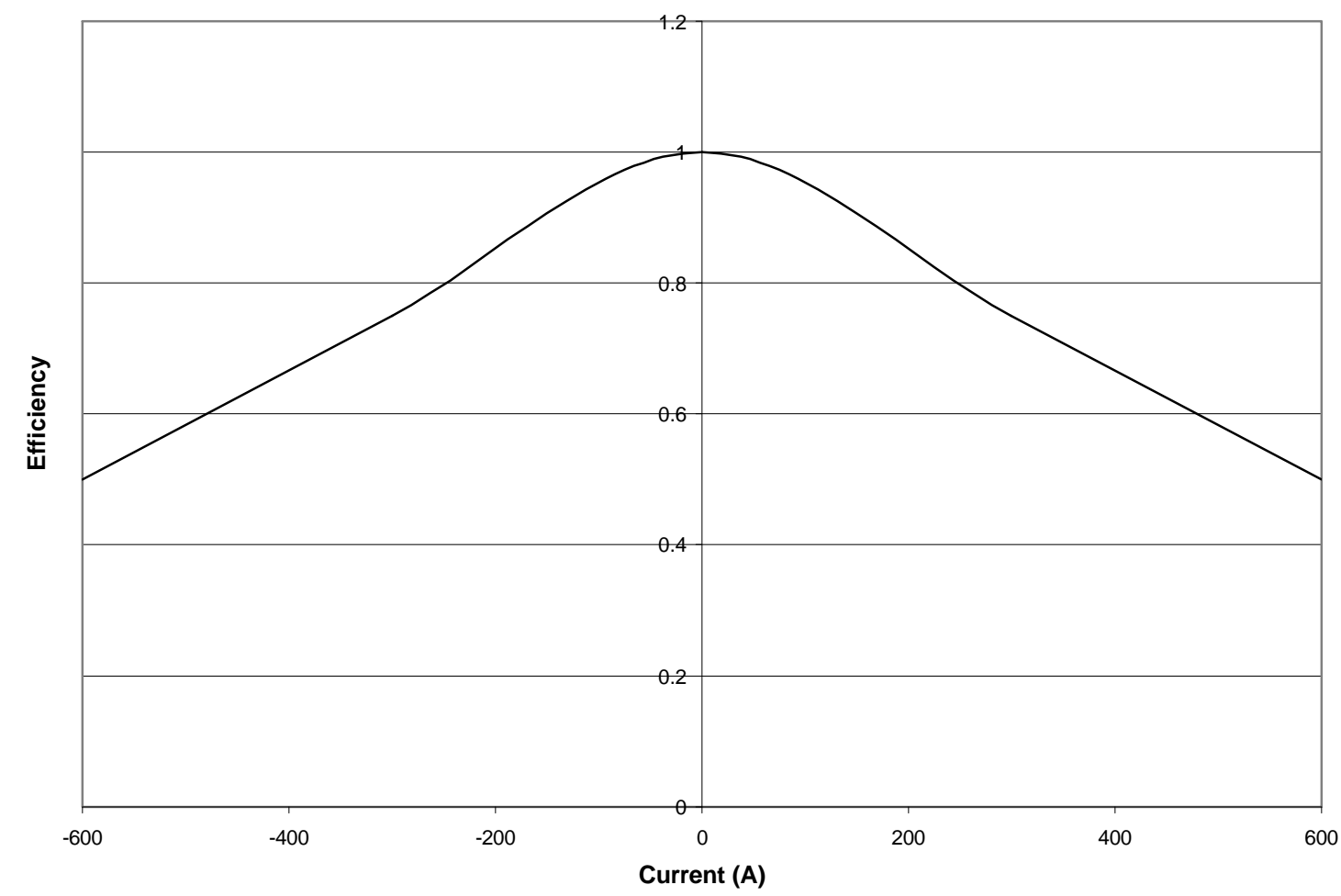

Figure 3.4.2 Battery efficiency vs. current. [17]

When power is demanded from the batteries by the electric motor, this efficiency factor causes a greater power draw from the batteries.

$$
P_{b}=\frac{P_{m}}{\eta_{b}}
$$


When power is being delivered to the batteries during regenerative braking or charging while driving, the efficiency factor decreases the power available to the battery below that delivered from the electric motor.

$$
P_{b}=\eta_{b} P_{m}
$$

Since the power flow at the batteries is known, and,

$$
1 \text { joule }=1 \frac{\text { watt }}{\sec \text { ond }}
$$

a new SoC is calculated from,

$$
S o C_{i}=\frac{S o C_{i-1} E_{b}-E_{b i}}{E_{b}}=\frac{S o C_{i-1} E_{b}-P_{b i}}{E_{b}}
$$

Once the new SoC is calculated, the control strategy determines a new power level for the ICE and motor based on the road load power, the target SoC, and the constants, $\mathrm{C}_{1}$ and $\mathrm{C}_{2}$. 


\section{Simulation Results}

To allow for comparison of the results for different vehicle configurations and cycle, for each value of $\mathrm{C}_{1}, \mathrm{C}_{2}$ was adjusted until the battery SoC at the end of the cycle was equal to the initial SoC. This is referred to as charge sustaining operation. $\mathrm{C}_{2}$ governs the SoC dependence of the engine. If $\mathrm{C}_{2}$ is high, the engine power will increase a large amount relative to the difference between the $\mathrm{SoC}$ and the target SoC. When the SoC climbs above the target $\mathrm{SoC}$, the $\mathrm{C}_{2}$ correction factor decreases the engine power to increase power demand on the electric motor and batteries. When the SoC falls below the target SoC, the correction factor increases the engine power reducing demand on the batteries and, in some instances, providing energy to the batteries through charging while driving. The initial and target $\mathrm{SoC}$ was set at $80 \%$ for all simulations. If the initial SoC was set at $100 \%$, not only would this be an unrealistic expectation for a charge sustaining hybrid, but there would be no capacity for recapturing regenerative braking energy until the batteries had been somewhat depleted.

In consideration of the space required to present all the final data from simulation and optimization, only selected figures will be presented. The remaining figures are presented in the Appendix and all of the results will be addressed in discussion. 


\subsection{Simulation Results}

Table 4.1.1 Conventional Vehicle uncorrected fuel economy from simulation.

\begin{tabular}{|c|c|c|c|c|c|c|}
\cline { 2 - 7 } \multicolumn{1}{c|}{} & \multicolumn{3}{c|}{ w/o auxiliary load } & \multicolumn{3}{c|}{ w/ auxiliary load } \\
\hline Cycle & Class 2B & Class 6 & Class 8 & Class 2B & Class 6 & Class 8 \\
\hline & \multicolumn{3}{|c|}{$\mathrm{mpg}$} & & \multicolumn{3}{c|}{$\mathrm{mpg}$} \\
\hline Freeway & 5.30 & 9.52 & 5.70 & 4.20 & 6.61 & 3.83 \\
\hline CSHVR & 3.55 & 5.32 & 3.07 & 1.99 & 2.73 & 1.61 \\
\hline Yard & 1.06 & 1.48 & 0.93 & 0.41 & 0.54 & 0.33 \\
\hline Manhattan & 2.06 & 3.12 & 1.89 & 0.84 & 1.16 & 0.71 \\
\hline Test D & 4.81 & 8.24 & 4.69 & 2.31 & 3.35 & 1.97 \\
\hline Combined & 3.97 & 6.42 & 3.80 & 2.23 & 3.18 & 1.89 \\
\hline
\end{tabular}

Table 4.1.2 Average Fuel Economy for In-use Conventional Vehicles [18, 19]

\begin{tabular}{|c|c|}
\cline { 2 - 2 } \multicolumn{1}{c|}{} & Fuel Economy \\
\cline { 2 - 2 } \multicolumn{1}{c|}{} & $\mathrm{mpg}$ \\
\hline Class 2 & 15.0 \\
\hline Class 6 & 7.3 \\
\hline Class 8 & 5.5 \\
\hline Class 8 Buses & 2.0 \\
\hline
\end{tabular}

Table 4.1.1 and 4.1.2 compare fuel economy for actual in-use and simulated conventional vehicles. If the in-use fuel economy figures for Class 8 are compared to the simulation results for the Freeway Cycle, they agree well. The average fuel economies in Table 4.1.2 are based on the total miles traveled and the total fuel consumed by all the vehicles in that class. The Class 8 average would tend to be weighted toward the fuel economy of OTR tractors due to the high number of miles they accumulate. If the Class 8 numbers are compared to the simulation results from the Freeway Cycle, they agree well. Similarly, the simulation results from the Manhattan Cycle are within 3\% of the average Class 8 bus fuel economy. On average, the Class 6 simulation results agree fairly well with the average in-use fuel economy. Although the fuel economy shown for 
average in-use Class 2 vehicles is most likely for unloaded vehicles, there is very little agreement between the Class $2 \mathrm{~B}$ simulation results and the actual vehicle average fuel economy. This is due to the use of driving cycles unsuitable for Class 2B vehicles. The chosen cycles are designed for heavy-duty vehicles and incorporate accelerations and speeds appropriate for their power and weight. As shown in Figure 3.1.4, Class 2B vehicles have a higher power to weight ratio allowing for increased dynamic performance. When these vehicles are simulated over heavy-duty drive cycles, the engine is run at low power and low efficiency resulting in very poor fuel economy prediction.

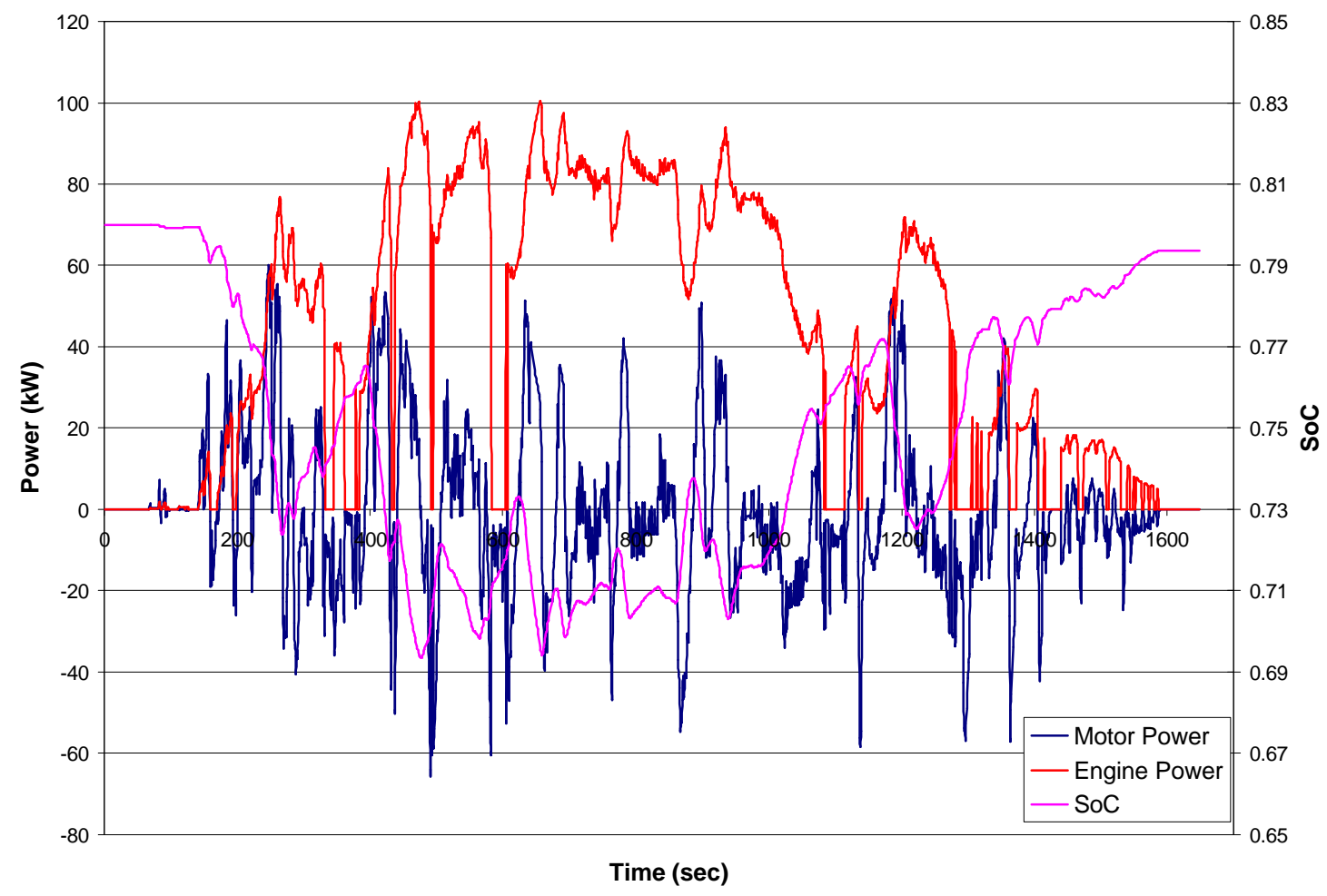

Figure 4.1.1 Class 6 Parallel HEV on Freeway Cycle without auxiliary load, $\mathrm{C}_{1}=$ $0.2, \mathrm{C}_{2}=3500$. 


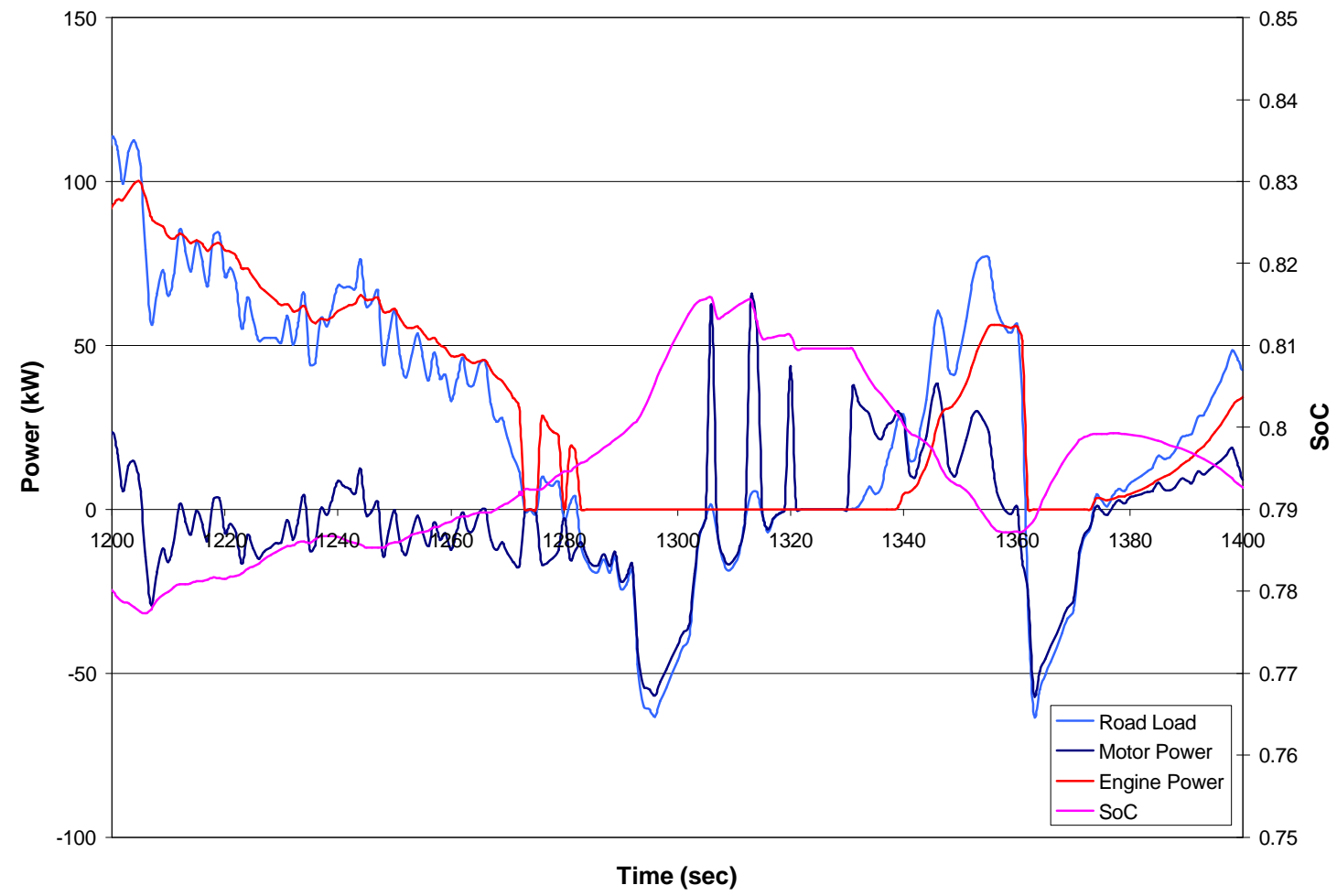

Figure 4.1.2 Expanded portion of Figure 4.1.1 from 1200 to 1400 seconds.

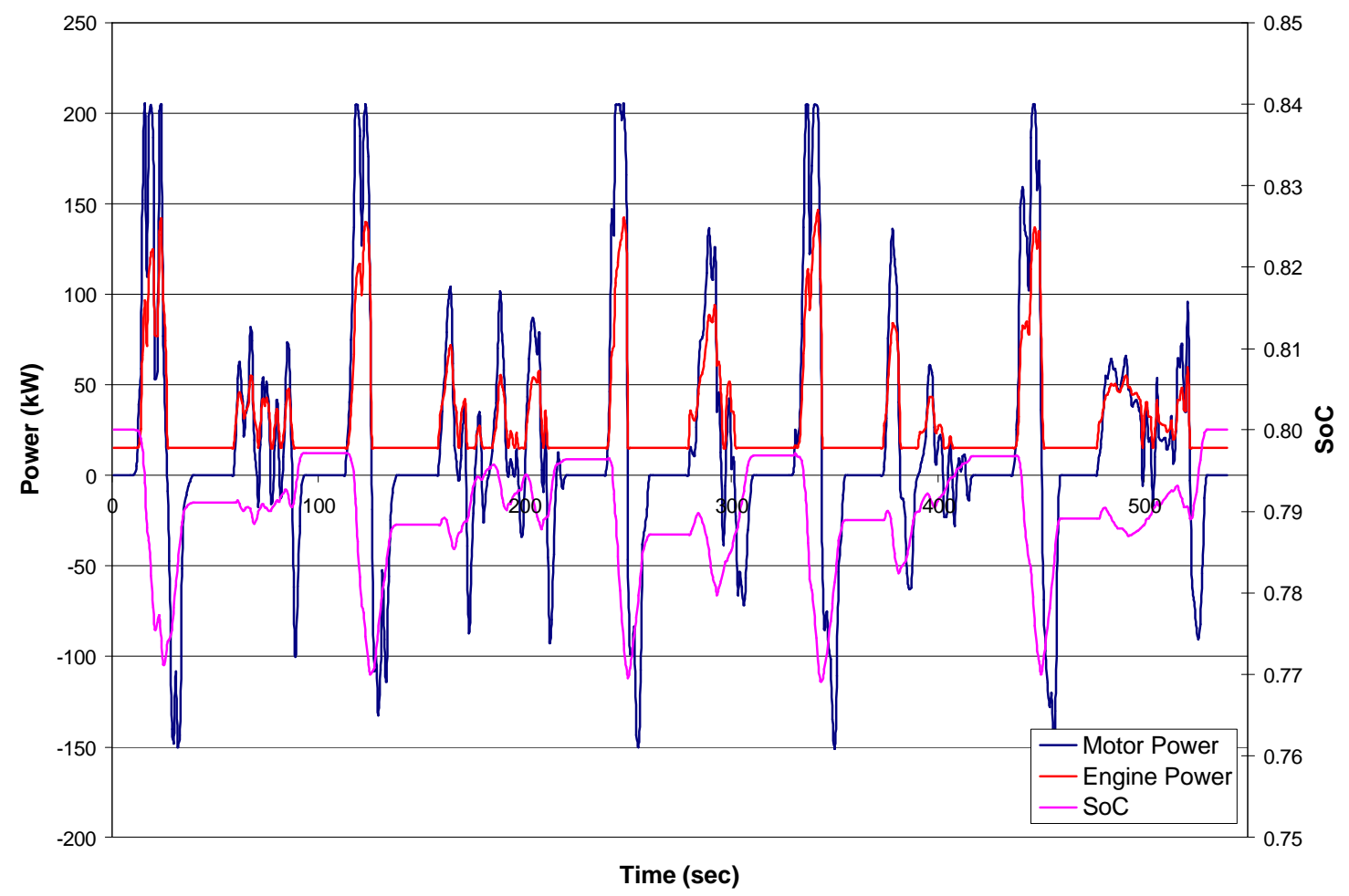

Figure 4.1.3 Class 8 Series HEV on Manhattan Cycle with auxiliary load, $\mathrm{C}_{1}=\mathbf{0 . 2}$, $\mathrm{C}_{2}=3375$. 


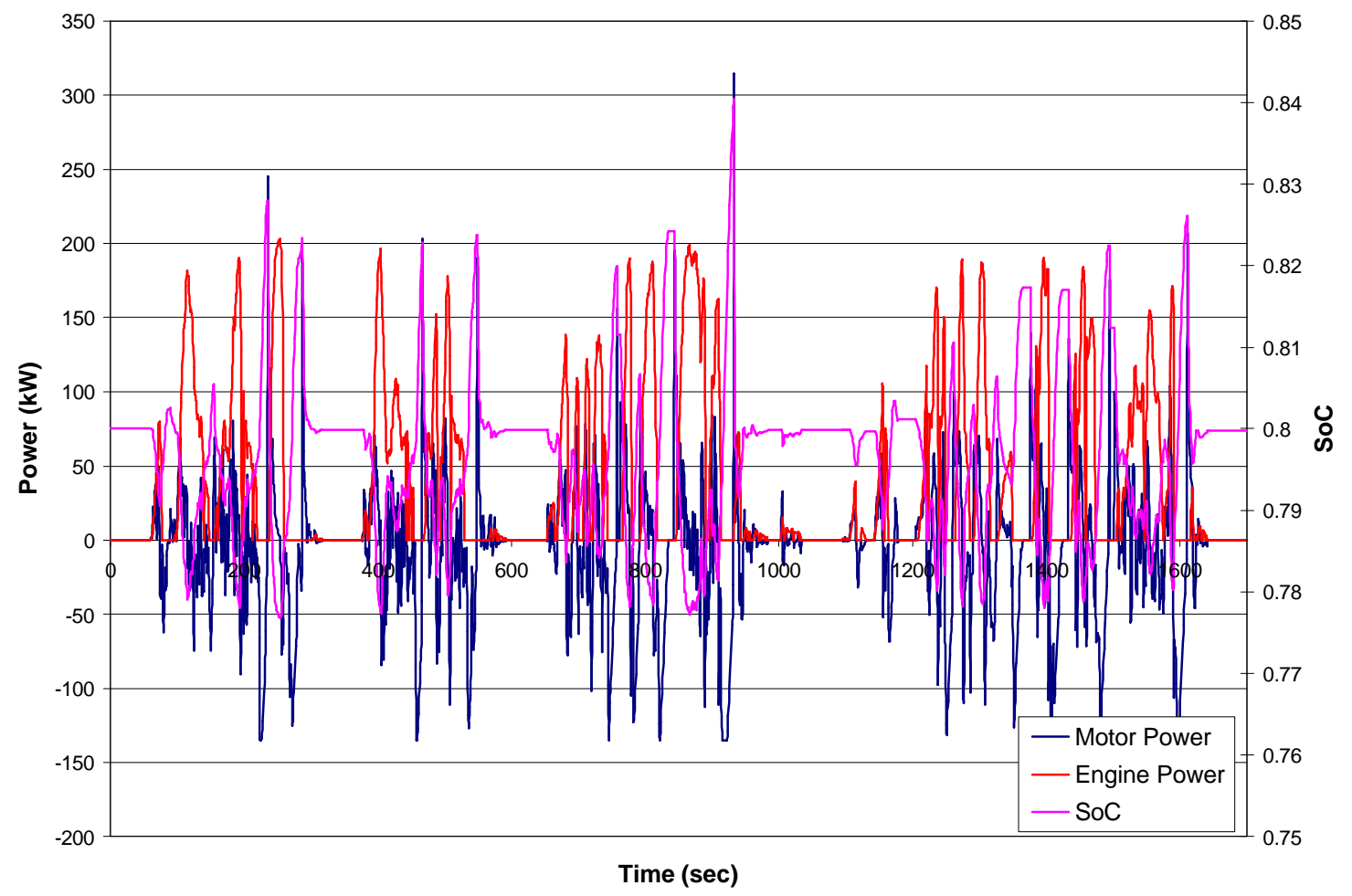

Figure 4.1.4 Class 8 Parallel HEV on CSHVC without auxiliary load, $\mathrm{C}_{1}=0.2, \mathrm{C}_{2}=$ 6800 .

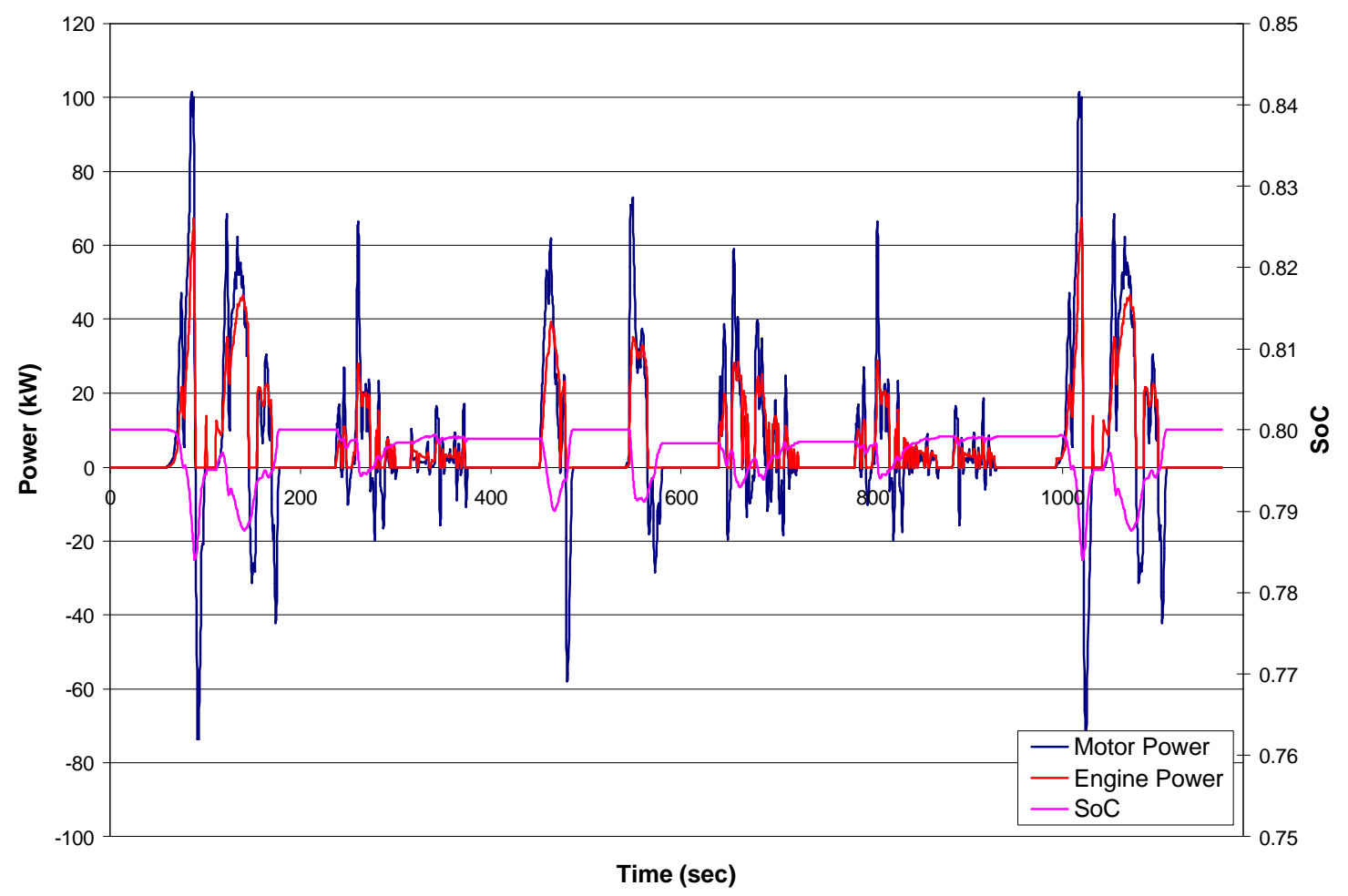

Figure 4.1.5 Class 8 Series HEV on Yard Cycle without auxiliary load, $\mathrm{C}_{1}=0.3, \mathrm{C}_{2}$ $=2550$. 


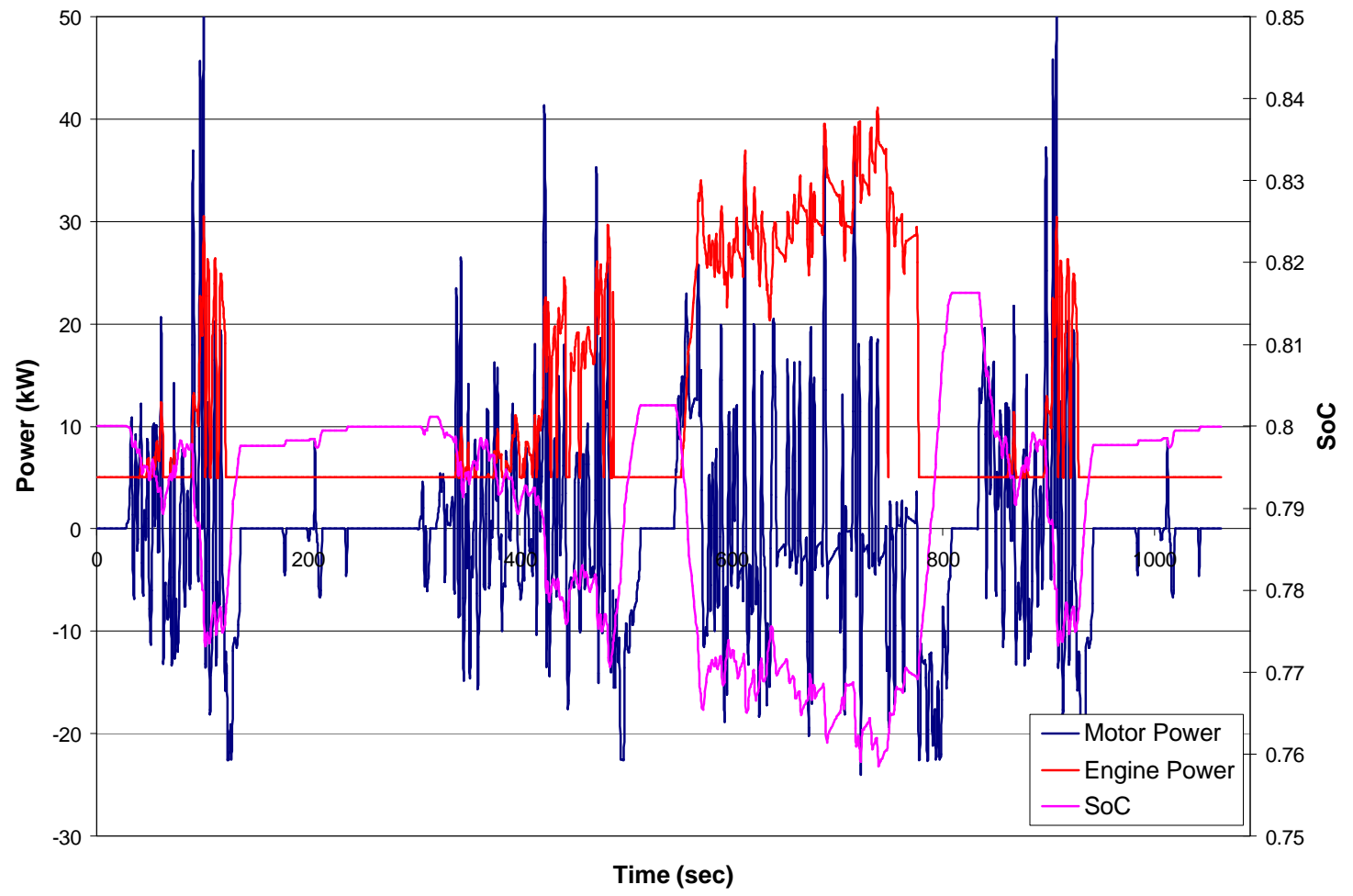

Figure 4.1.6 Class 2B Parallel HEV on Test D Cycle with auxiliary load, $\mathrm{C}_{1}=0.1$, $\mathrm{C}_{2}=1290$.

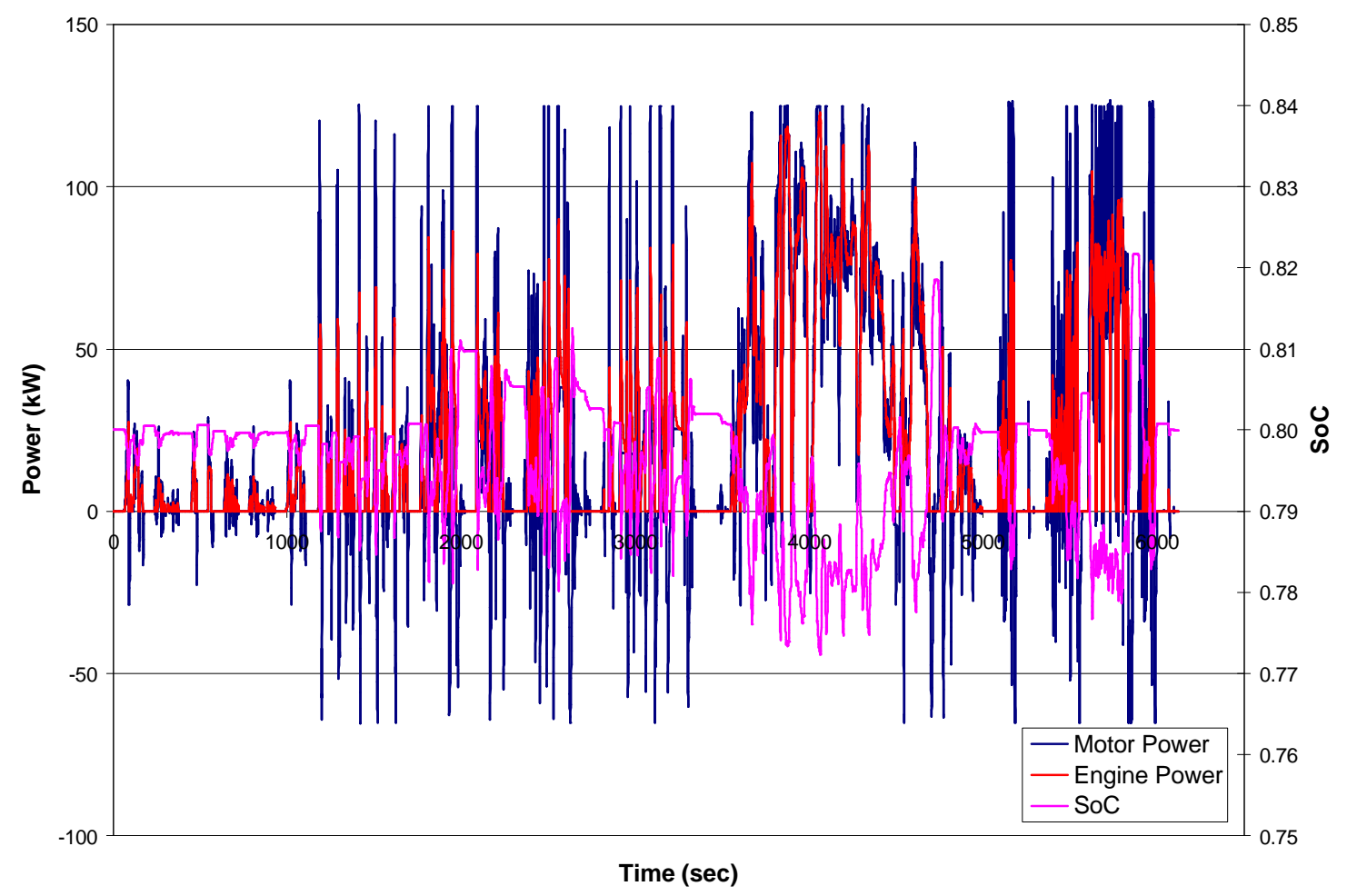

Figure 4.1.7 Class 6 Series HEV on Combined Cycle without auxiliary load, $\mathrm{C}_{1}=$ $0.2, \mathrm{C}_{2}=3550$. 


\subsection{Optimization}

Figures 4.2.1 through 4.2.18 show the variation of fuel economy with $\mathrm{C}_{1}$ for the various vehicle configurations and cycles. While most of the plots are fairly smooth and predictable, a deficiency of the simulation is readily apparent in Figures 4.2.2, 4.2.3 4.2.10, 4.2.15, and 4.2.18. In these figures, some of the fuel economy traces show dramatic changes for small changes in $C_{1}$ or are not complete for the entire range of $C_{1}$ values. This is due to the control strategy. In situations where $C_{2}$ is very large to maintain charge sustaining operation over the cycle, very small changes in SoC can result in large spikes and oscillations in engine power. This is due to the use of a control strategy that is directly proportional to the road load power and the SoC. A solution would be the inclusion of a derivative term shown in Equation 18.

$$
P_{e}=C_{1} P_{w}-C_{2}\left(S o C_{i}-S o C_{t}\right)+C_{3} \frac{d P_{e}}{d t}
$$

This modified equation can be used to limit the speed of change in engine power over a period of time and would serve as a damper to smooth out spikes and oscillations. This strategy could be implemented by imposing a maximum rate of change of engine power. $\mathrm{C}_{3}$ could be sized to set the level of damping required. 


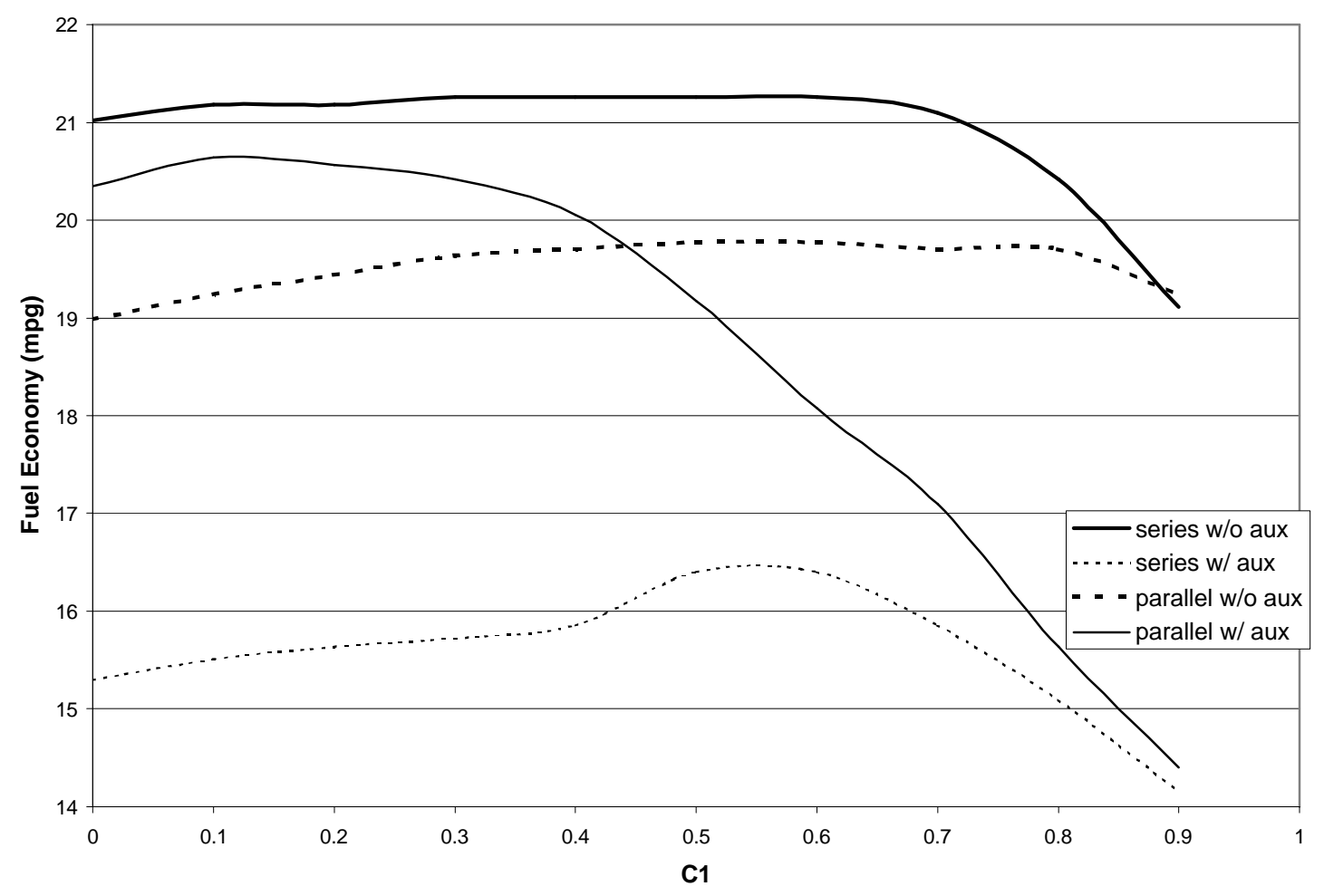

Figure 4.2.1 Class 2B HEV Fuel Economy on Freeway Cycle.

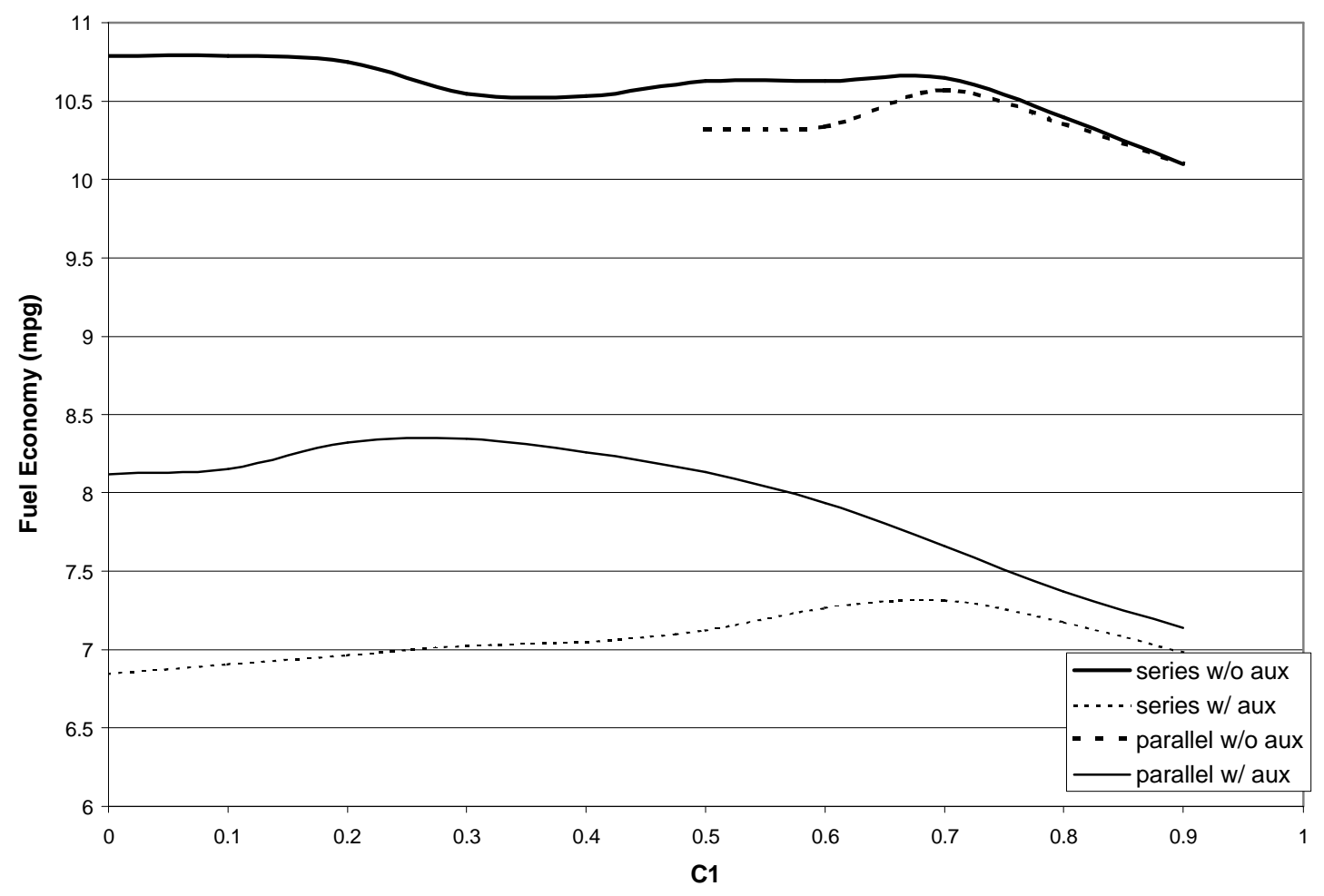

Figure 4.2.2 Class 6 HEV Fuel Economy on Freeway Cycle. 


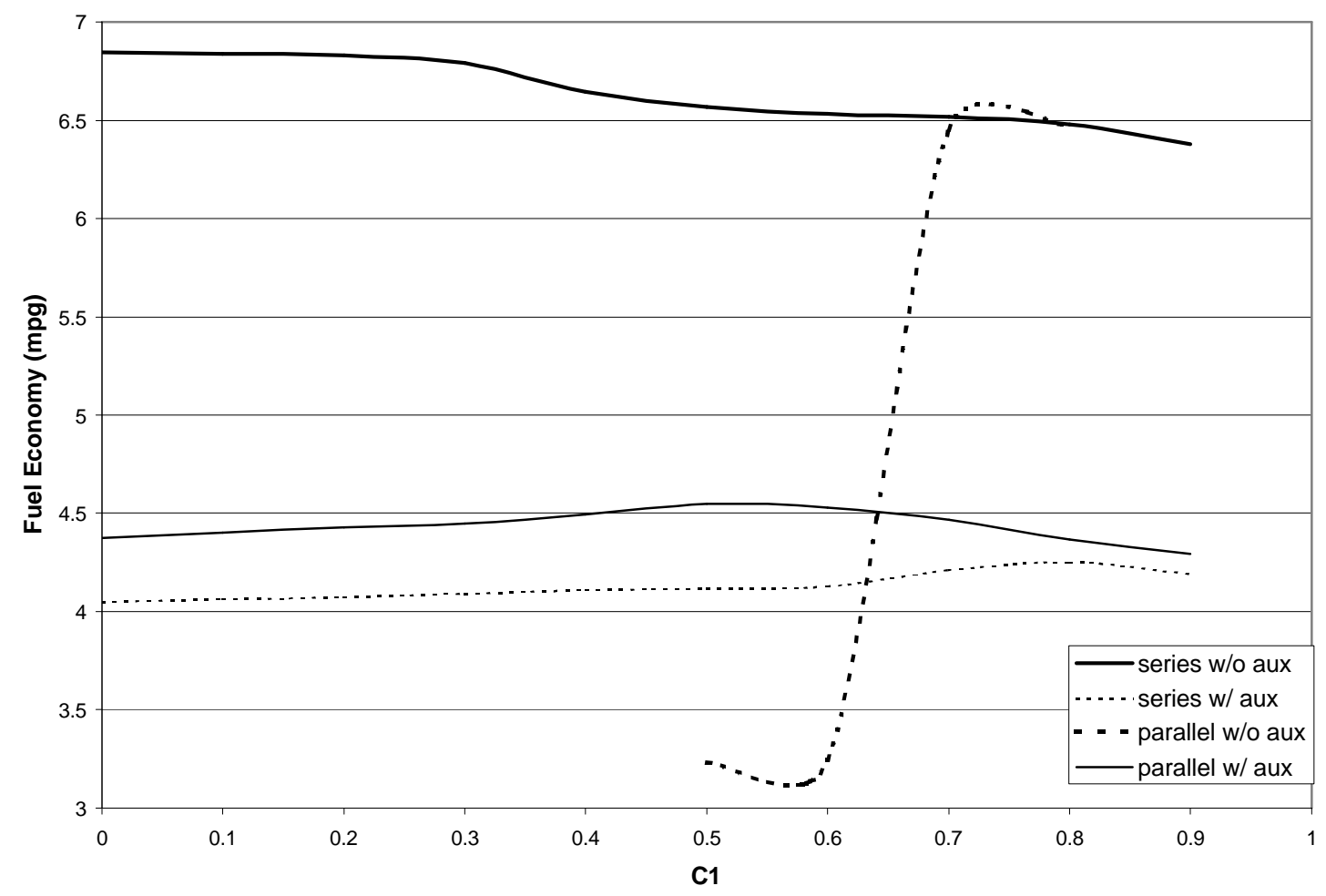

Figure 4.2.3 Class 8 HEV Fuel Economy on Freeway Cycle.

The instability in the parallel HEV without auxiliary load in Figure 4.2.3 as well as the incomplete Class 6 data in Figure 4.2.2 are due to the numerical instability in the simulation discussed at the beginning of this section. Similar instabilities can be seen in the Class 8 and Class 2B HEVs in Figures 4.2.6 and 4.2.7. 


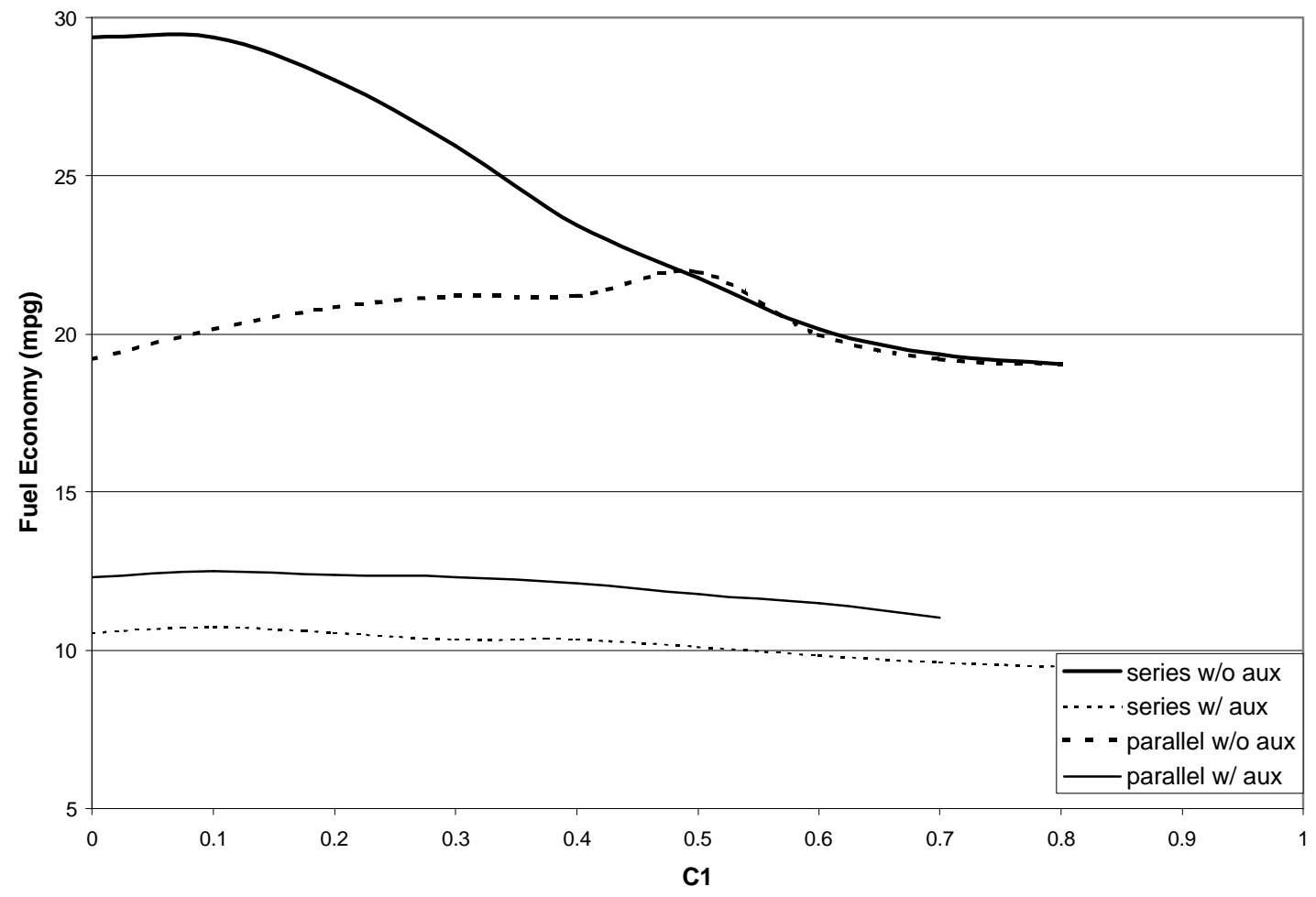

Figure 4.2.4 Class 2B HEV Fuel Economy on CSHVC Cycle.

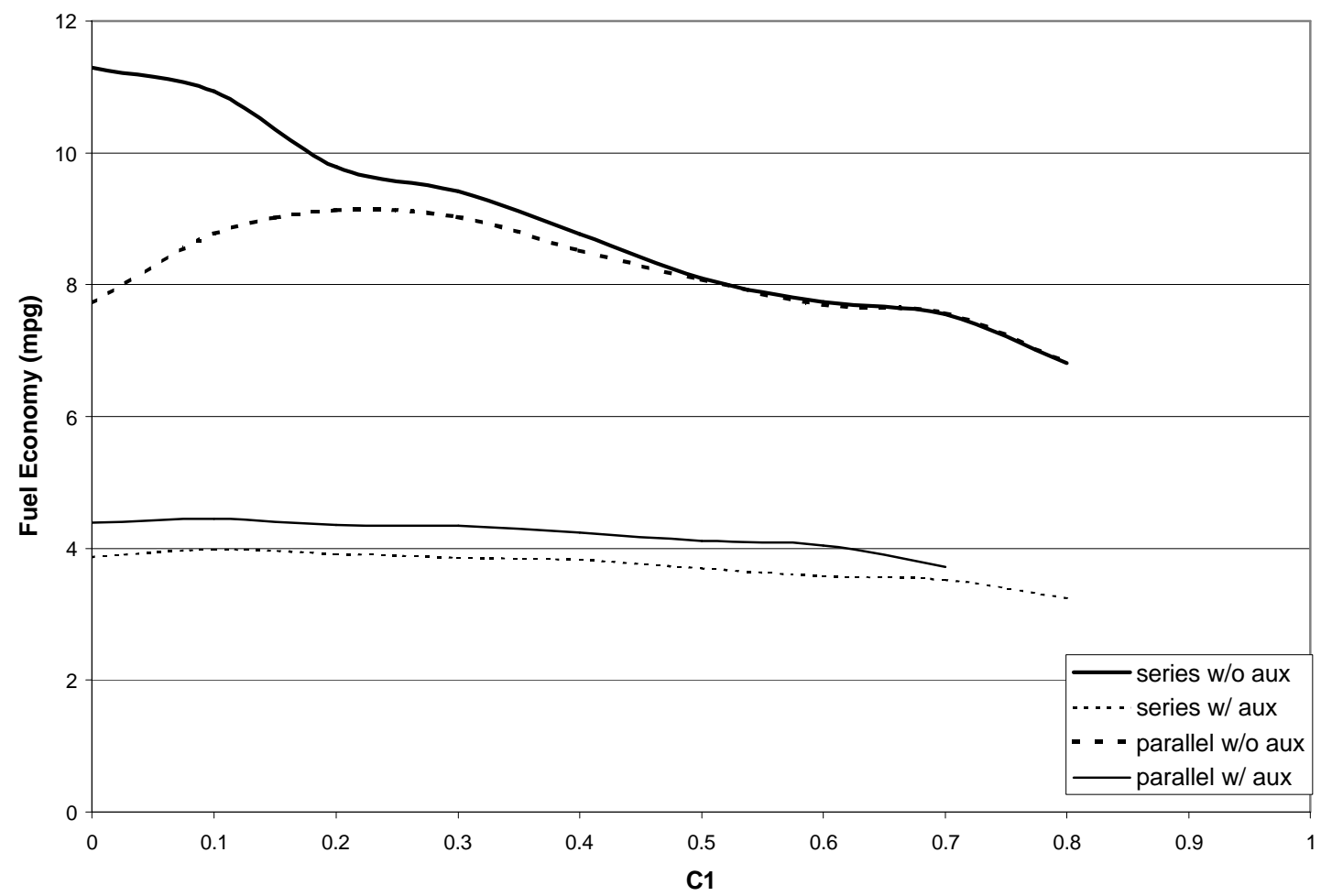

Figure 4.2.5 Class 6 HEV Fuel Economy on CSHVC Cycle. 


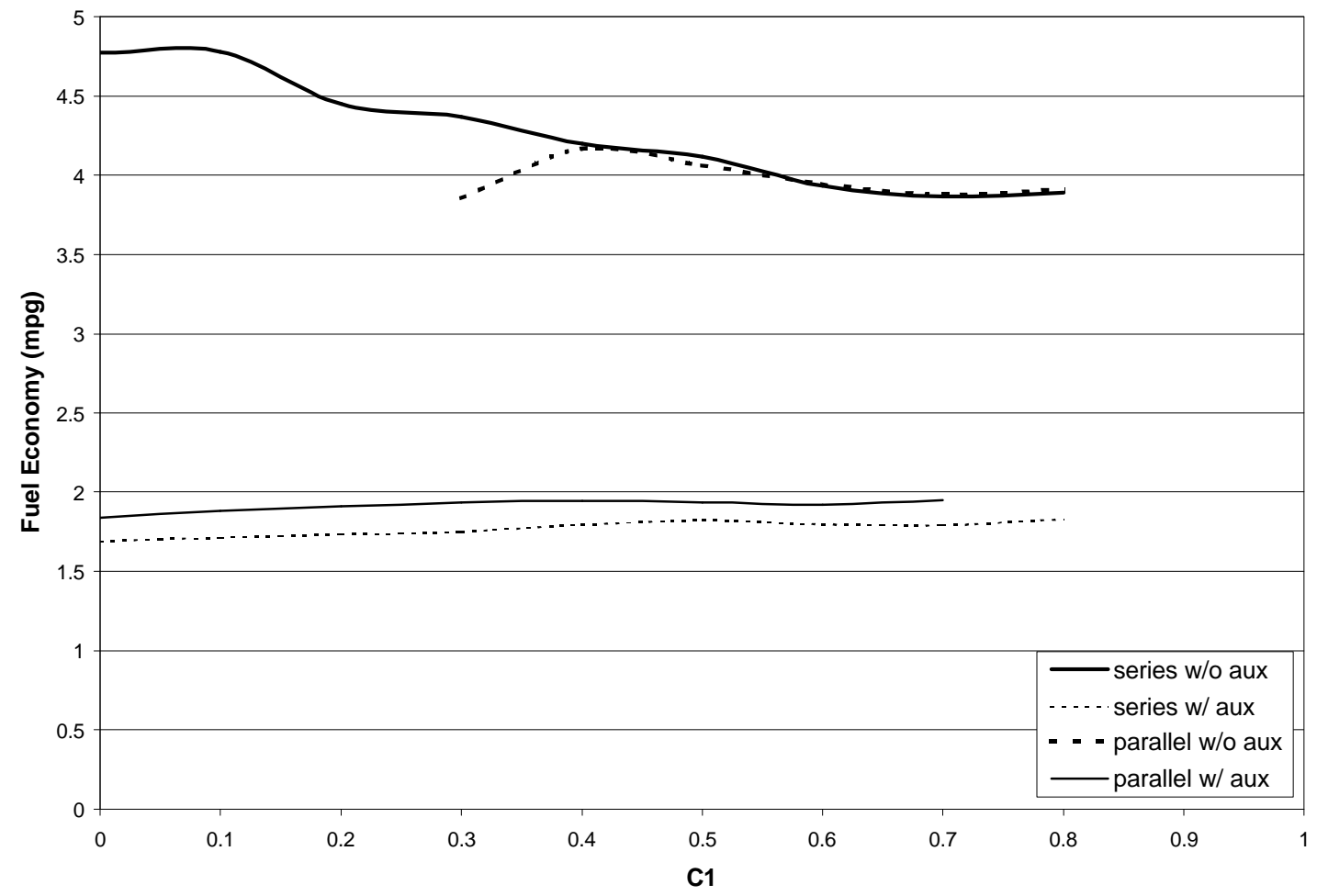

Figure 4.2.6 Class 8 HEV Fuel Economy on CSHVC Cycle.

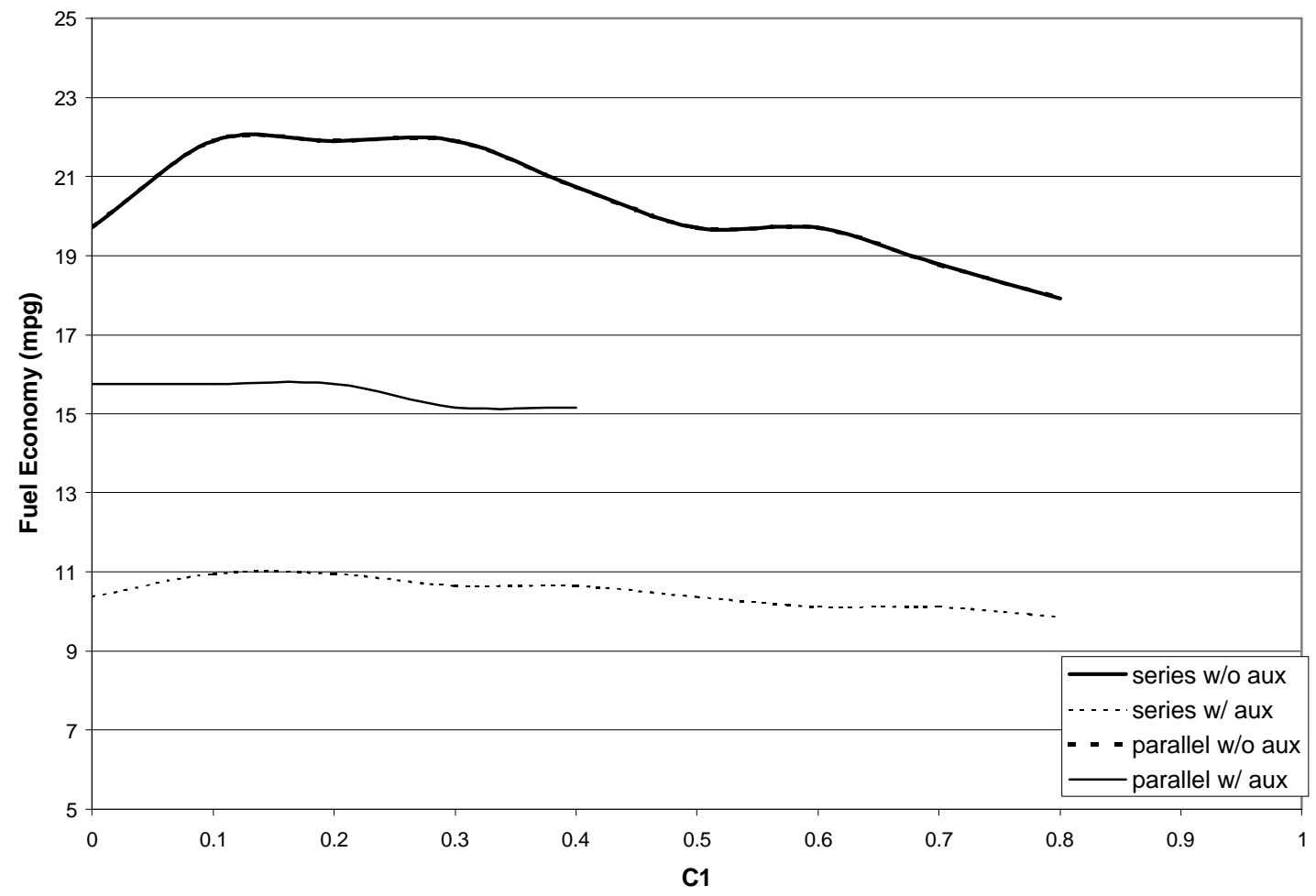

Figure 4.2.7 Class 2B HEV Fuel Economy on Yard Cycle. 
The data for the Class 8 parallel HEV in Figure 4.2.7 appears to be missing. This is due to overlapping data from the Class 8 series HEV.

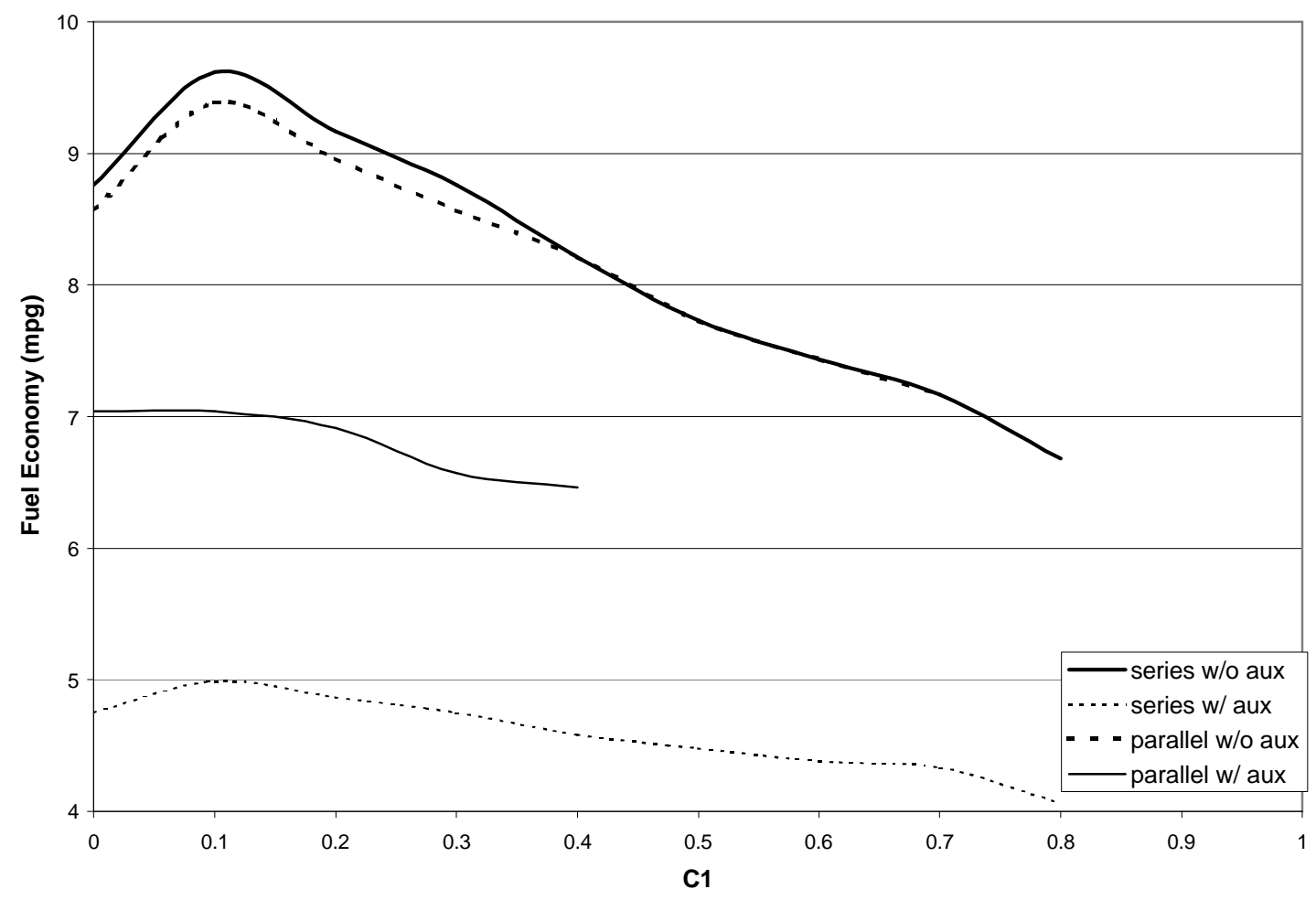

Figure 4.2.8 Class 6 HEV Fuel Economy on Yard Cycle. 


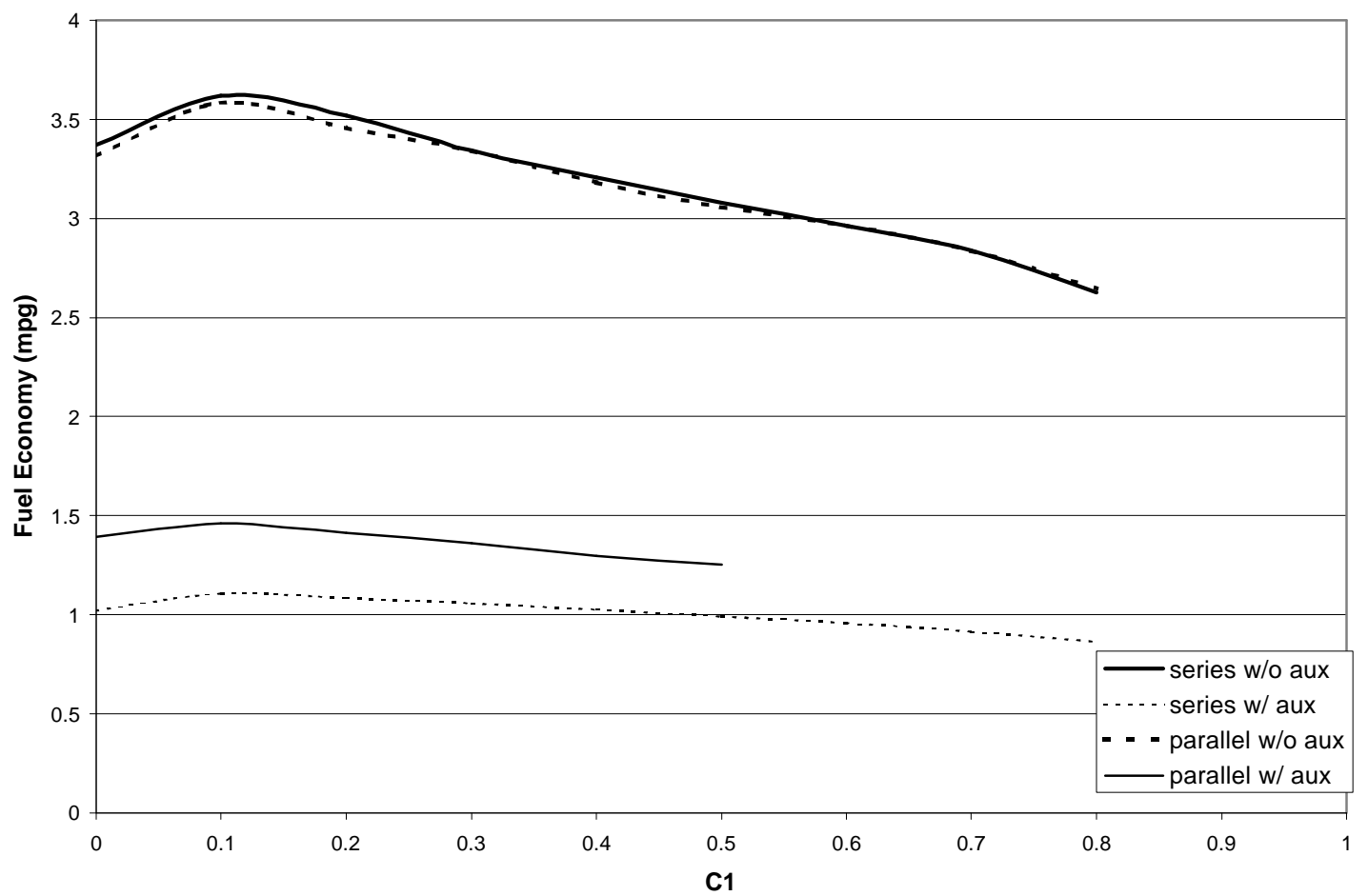

Figure 4.2.9 Class 8 HEV Fuel Economy on Yard Cycle.

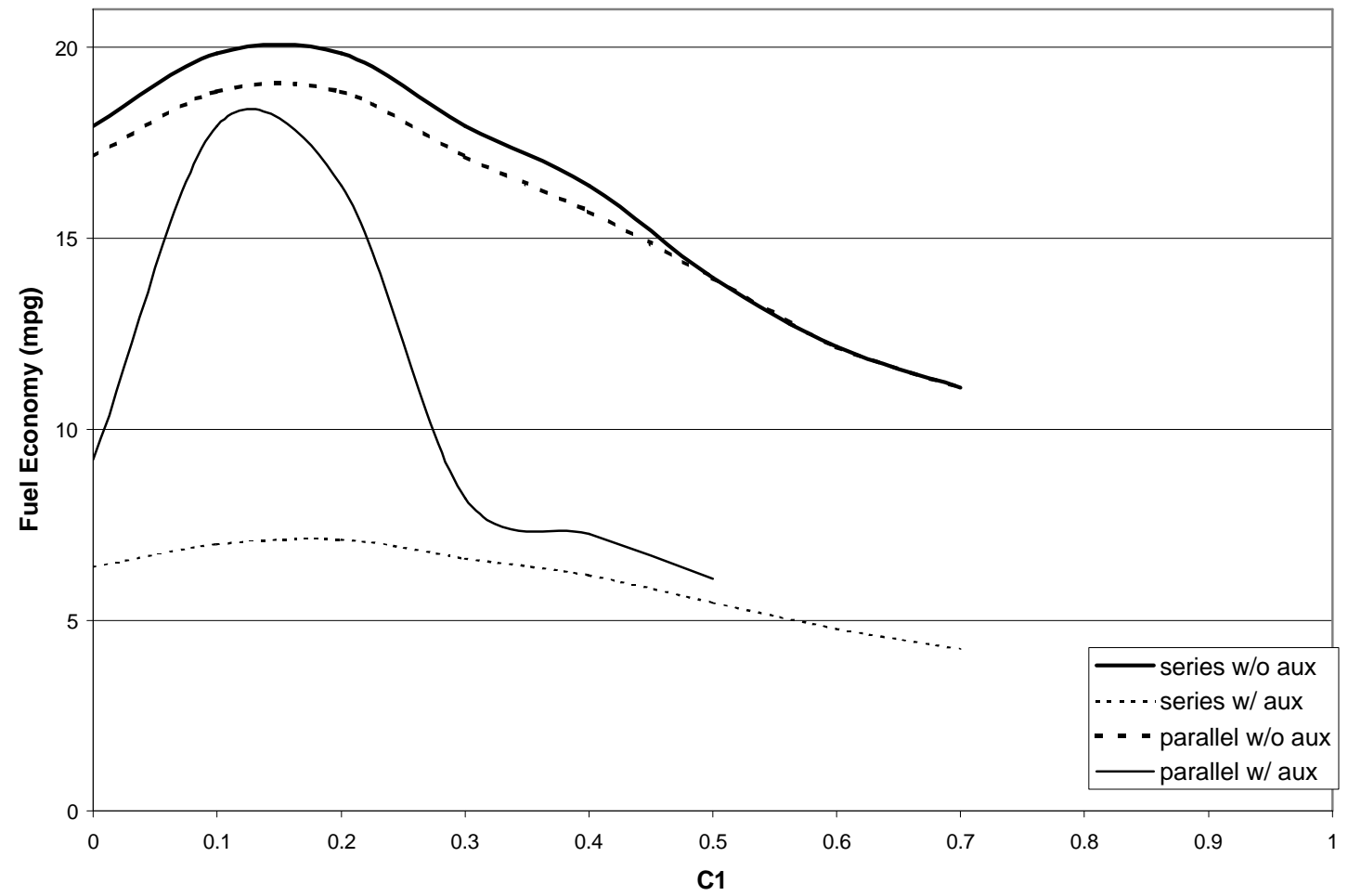

Figure 4.2.10 Class 2B HEV Fuel Economy on Manhattan Cycle. 


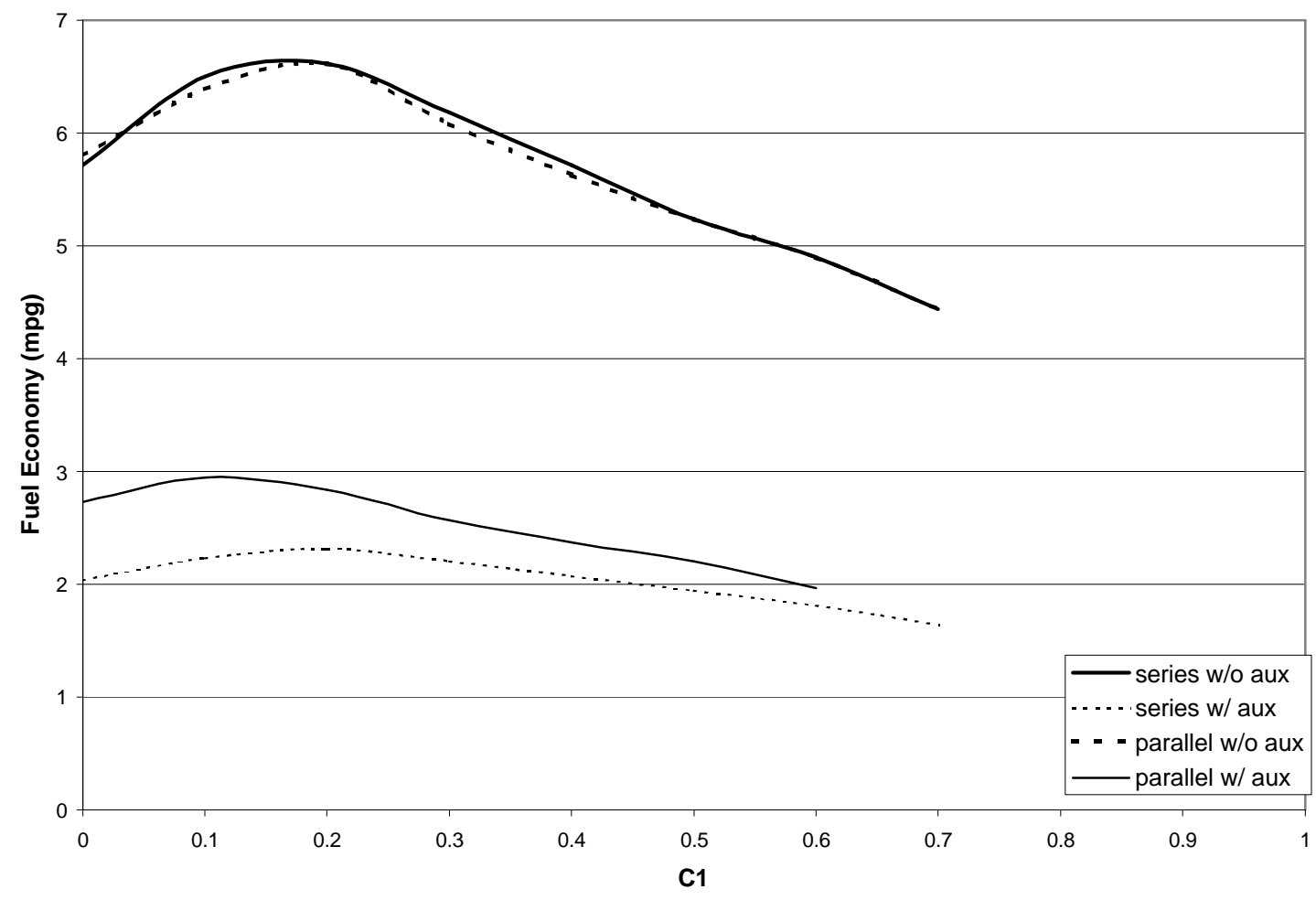

Figure 4.2.11 Class 6 HEV fuel economy on Manhattan Cycle.

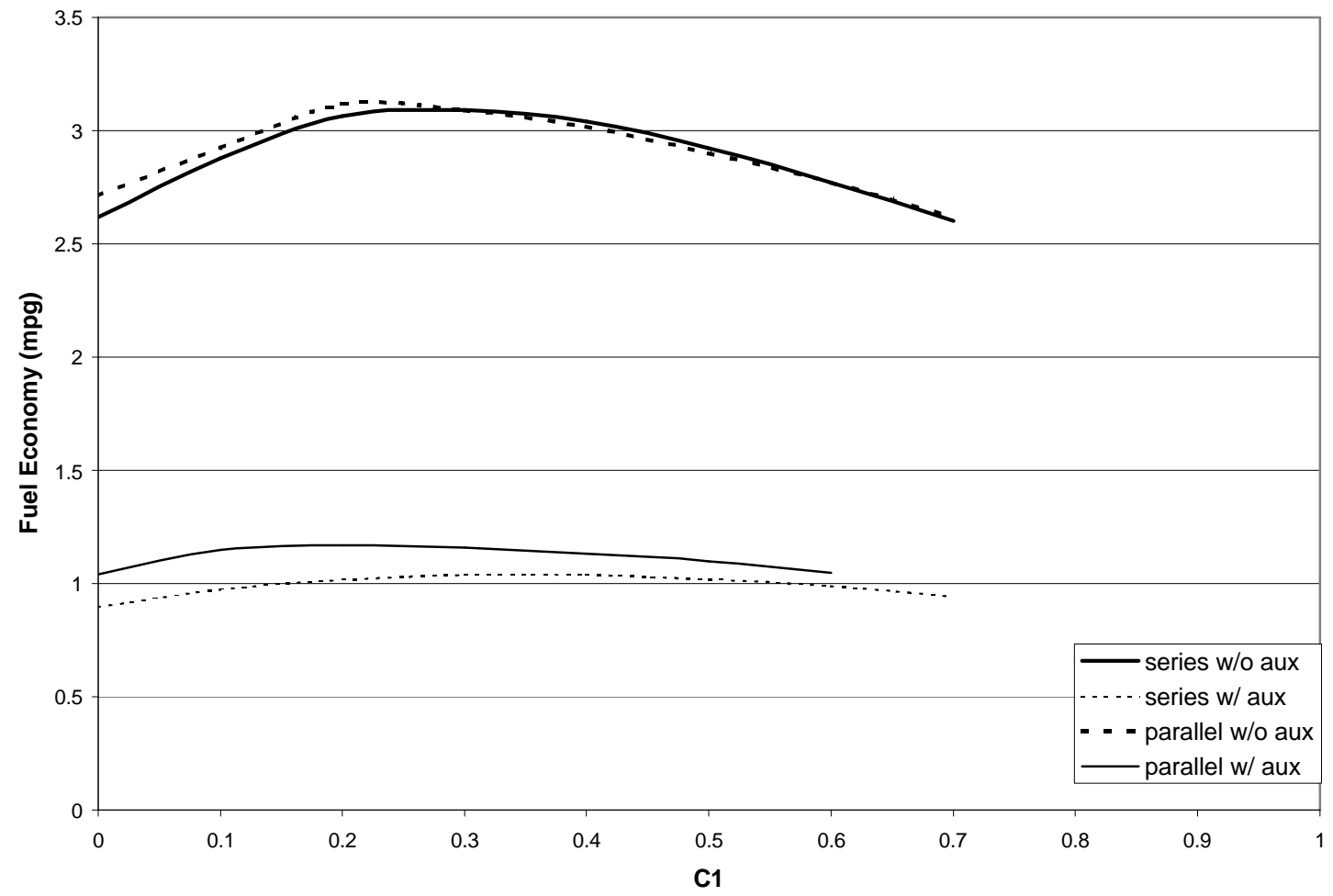

Figure 4.2.12 Class 8 HEV fuel economy on Manhattan Cycle. 


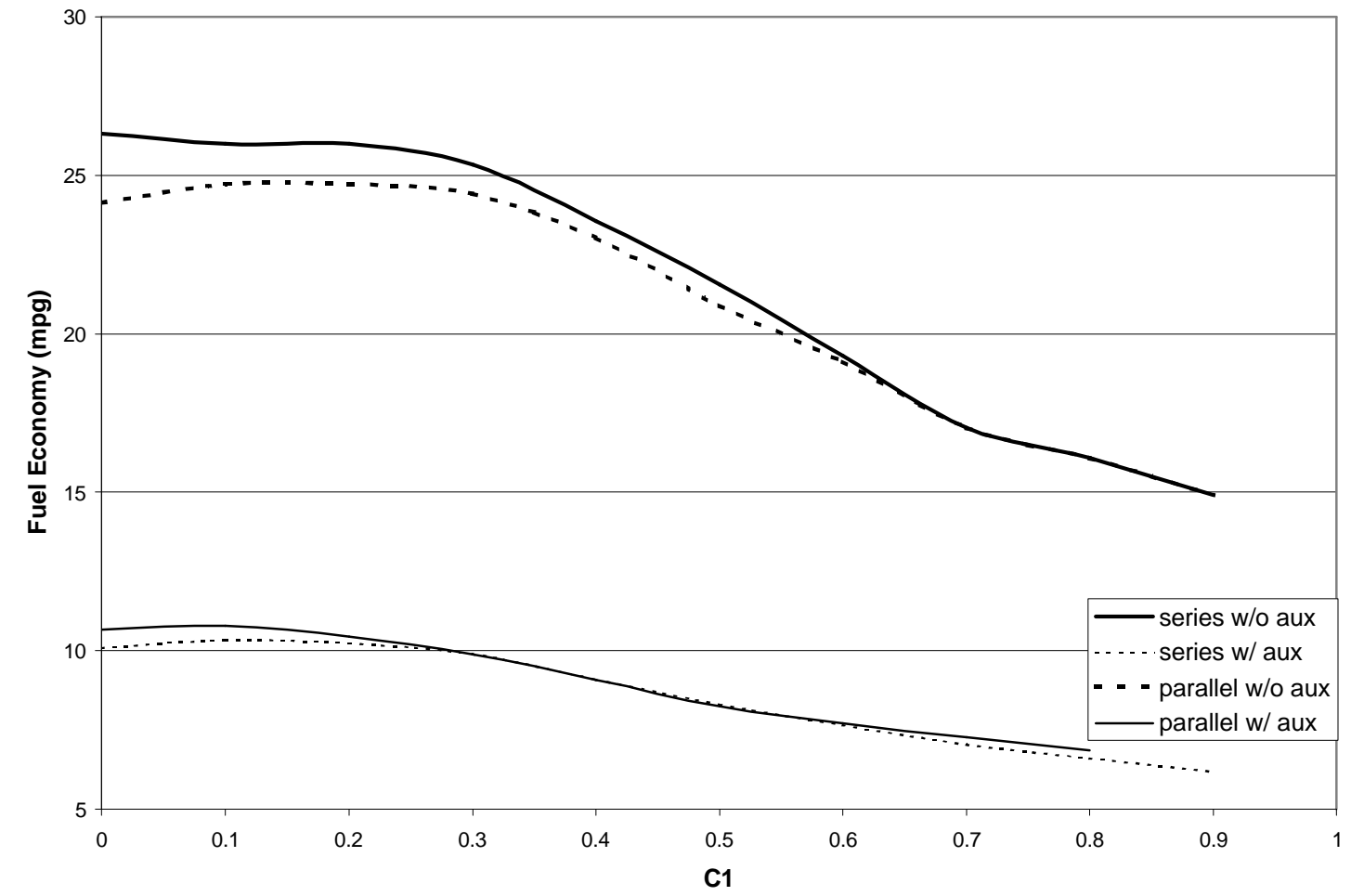

Figure 4.2.13 Class 2B HEV fuel economy on Test D Cycle.

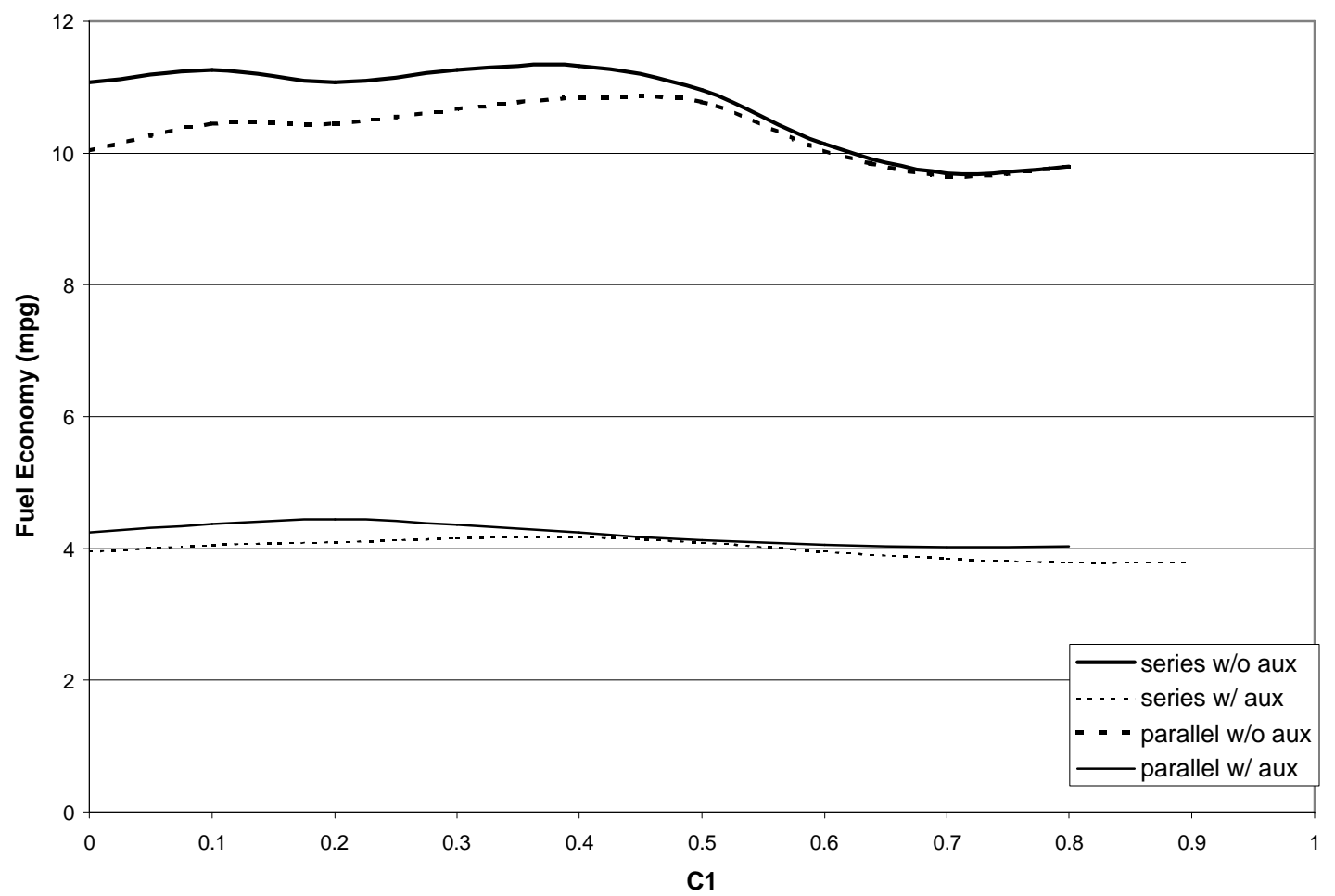

Figure 4.2.14 Class 6 HEV fuel economy on Test D Cycle. 


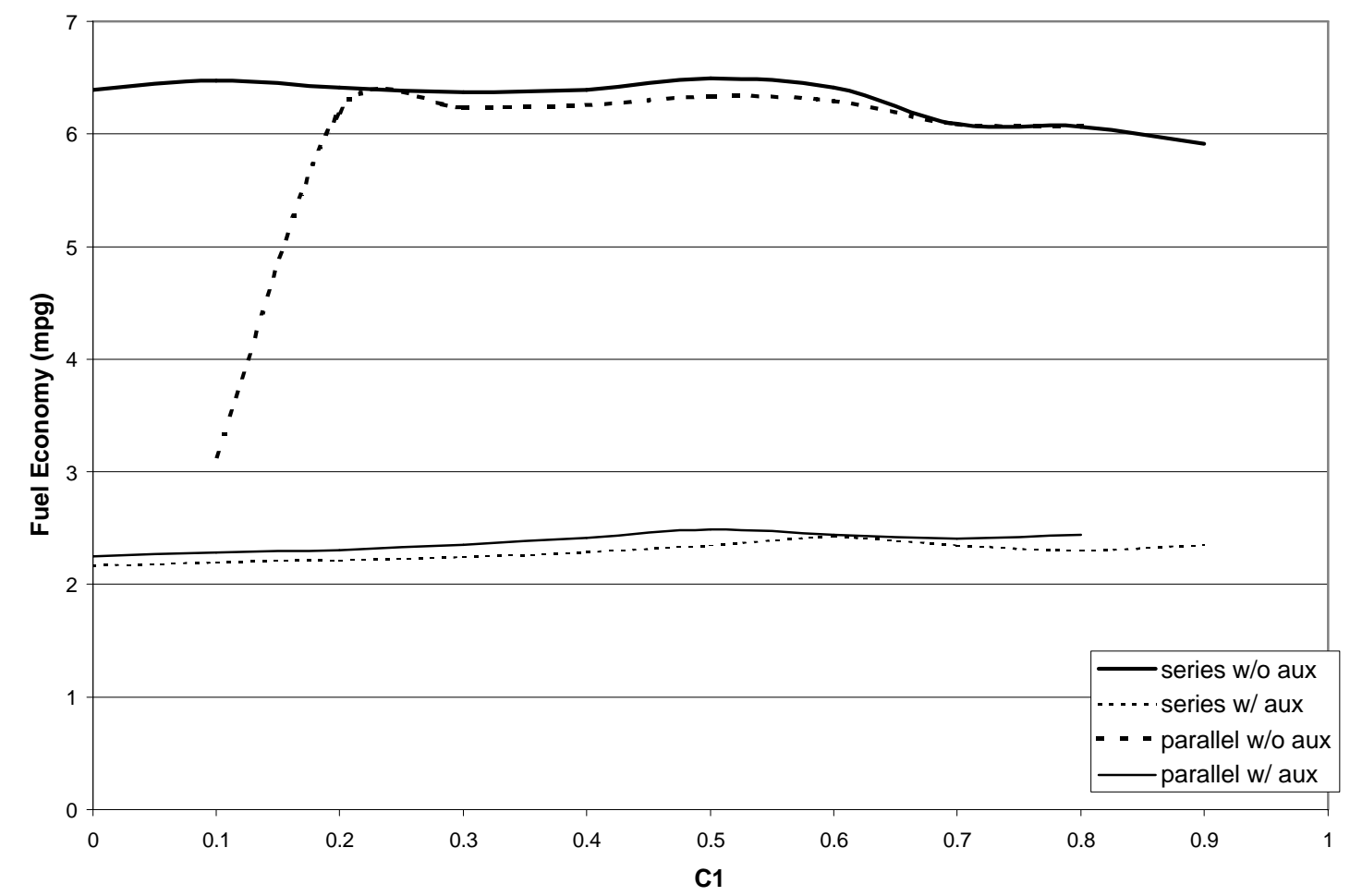

Figure 4.2.15 Class 8 HEV fuel economy on Test D Cycle.

The sudden instability of the parallel HEV without auxiliary loads as $\mathrm{C}_{1}$ approaches zero in Figures 4.2.15 and 4.1.18 are, again, due to the numerical instabilities in the simulation. Incorporating the changes recommended at the beginning of the section might solve these problems. 


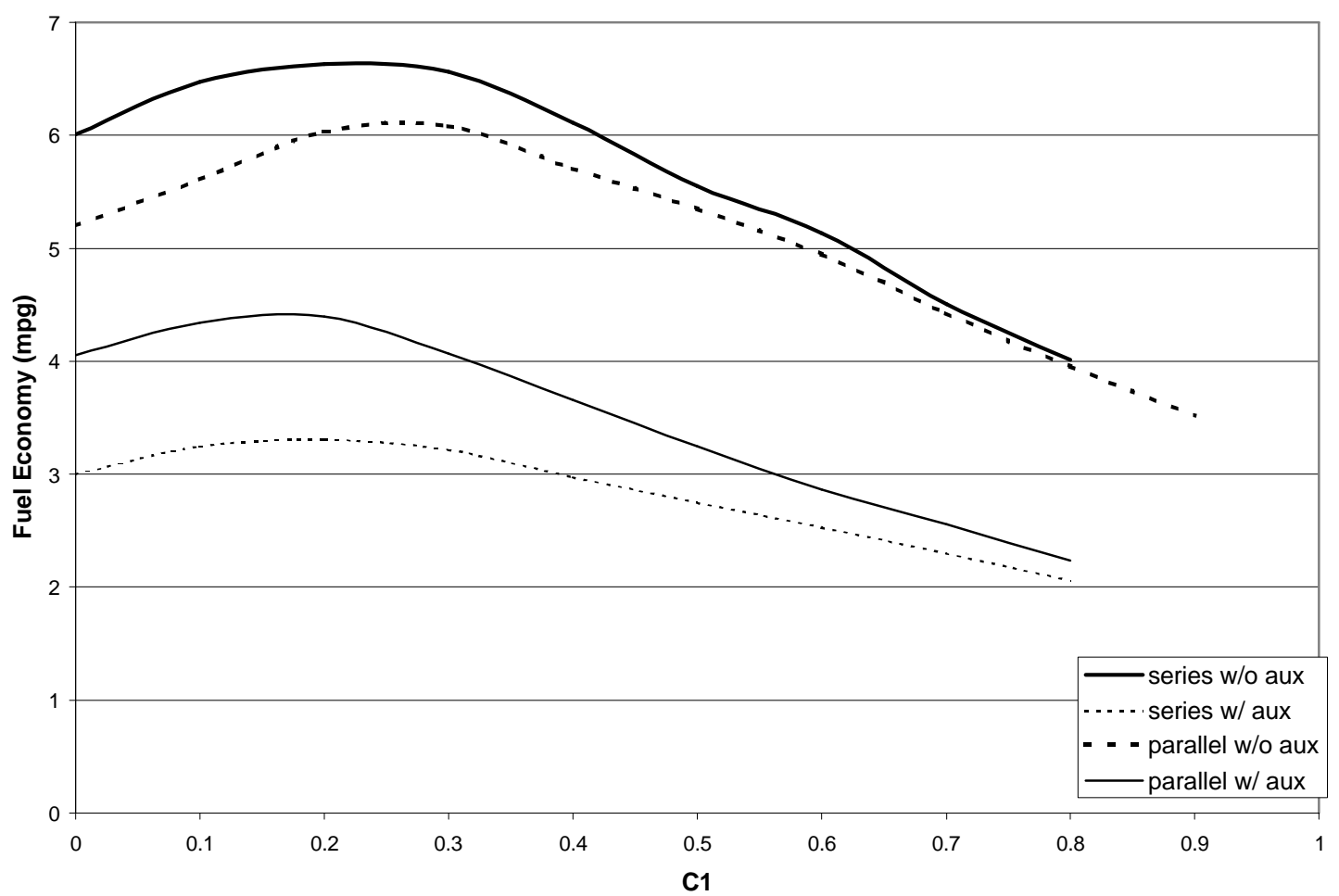

Figure 4.2.16 Class 2B HEV fuel economy on Combined Cycle.

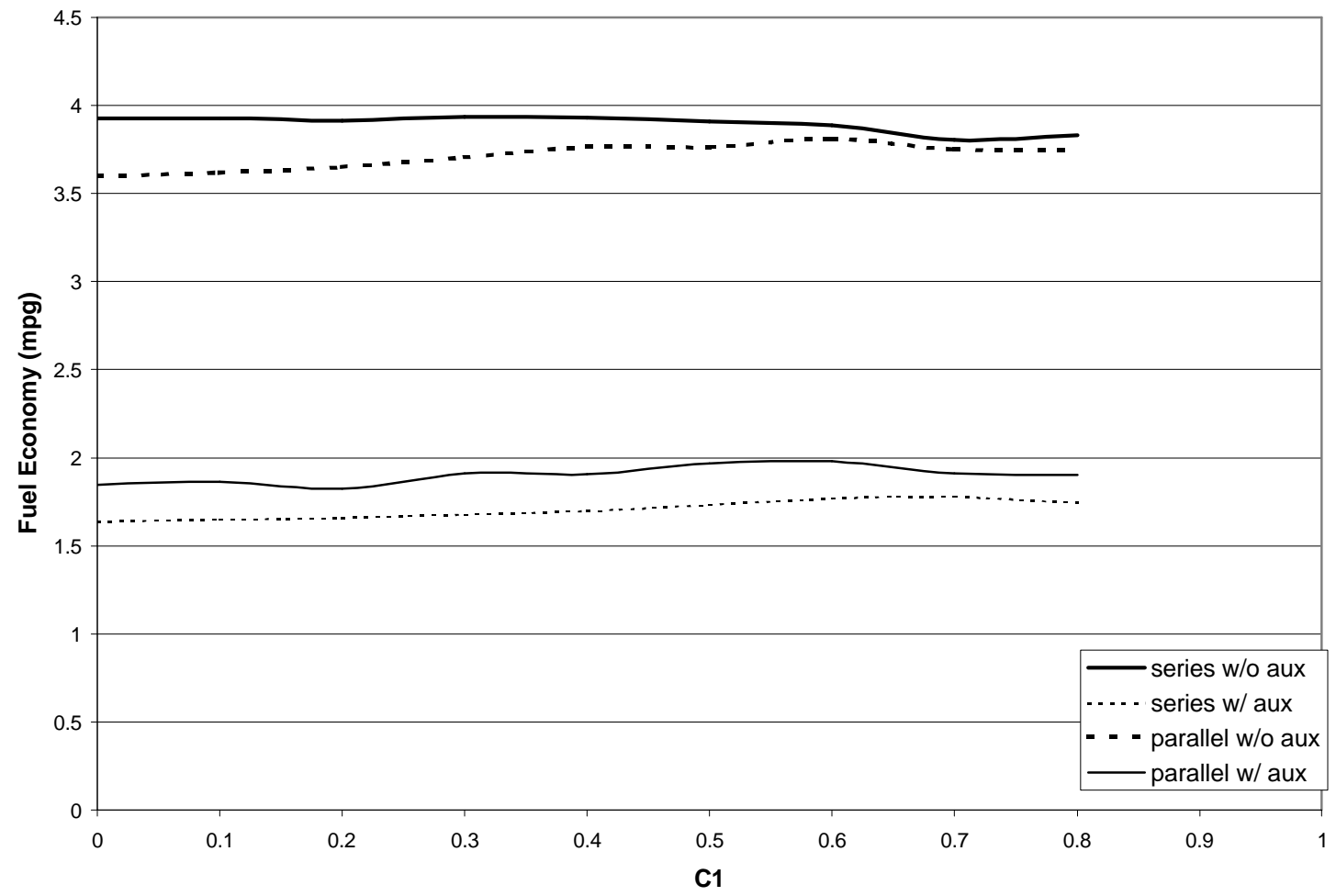

Figure 4.2.17 Class 6 HEV fuel economy on Combined Cycle. 


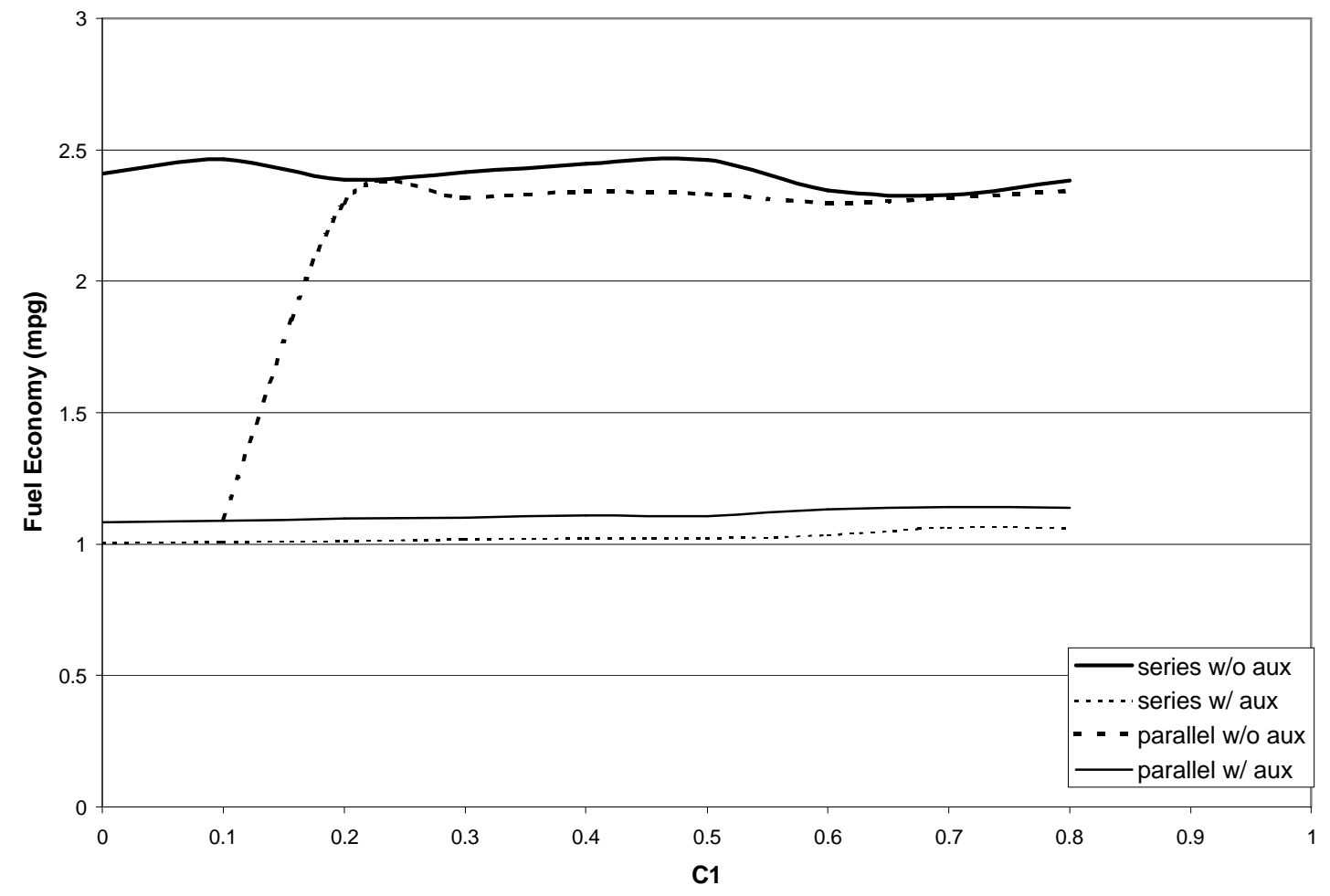

Figure 4.2.18 Class 8 HEV fuel economy on Combined Cycle.

Table 4.2.1 Class 2B optimum HEV without auxiliary load configurations and constants directly from simulation.

\begin{tabular}{|c|c|c|c|c|c|c|}
\hline Cycle & Best Fuel Economy & Hybrid Configuration & C1, C2 & $\%$ change from conventional & Engine Power & Motor Power \\
\hline & $\mathrm{mpg}$ & & & & $\mathrm{kW}$ & $\mathrm{kW}$ \\
\hline Freeway & 21.26 & Series & $0.5,2960$ & 301 & 55 & 66 \\
\hline CSHVC & 29.37 & Series & $0.1,1490$ & 90 & 32 & 41 \\
\hline Yard & 21.90 & Parallel & $0.3,1250$ & 1966 & 7 & 14 \\
\hline Manhattan & 19.84 & Series & $0.2,3070$ & 858 & 21 & 48 \\
\hline Test D & 12.98 & Series & $0.2,1230$ & 170 & 42 & 77 \\
\hline Combined & 6.63 & Series & $0.2,1230$ & 67 & 64 & 120 \\
\hline
\end{tabular}

Table 4.2.2 Class 2B optimum HEV with auxiliary load configurations and constants directly from simulation.

\begin{tabular}{|c|c|c|c|c|c|c|}
\hline Cycle & Best Fuel Economy & Hybrid Configuration & $\mathrm{C} 1, \mathrm{C} 2$ & \% change from conventional & Engine Power & Motor Power \\
\hline & $\mathrm{mpg}$ & & & & $\mathrm{kW}$ & $\mathrm{kW}$ \\
\hline Freeway & 20.42 & Parallel & $0.3,225$ & 396 & 41 & 33 \\
\hline CSHVC & 12.50 & Parallel & $0.1,540$ & 60 & 28 & 29 \\
\hline Yard & 15.77 & Parallel & $0.2,405$ & 3746 & 5 & 12 \\
\hline Manhattan & 17.95 & Parallel & $0.1,660$ & 2037 & 43 & 16 \\
\hline Test D & 10.45 & Parallel & $0.2,750$ & 352 & 41 & 52 \\
\hline Combined & 4.40 & Parallel & $0.2,750$ & 97 & 51 & 78 \\
\hline
\end{tabular}

The results of the simulation overstate the percent increase in fuel economy for a Class 2B HEV over a conventional vehicle. While the conventional vehicle fuel 
economy determined from simulation of the Class 6 and 8 vehicles is similar to that seen in actual vehicles, the fuel economy for the Class $2 \mathrm{~B}$ vehicles is far lower that what is expected from actual in-use vehicles due to the use of heavy-duty cycles. If the HEV results from the simulation are compared to more realistic conventional fuel economy, the percent improvement is much lower as shown in Table 4.2.3

Table 4.2.3 Class 2B without auxiliary load simulation results compared to actual vehicle fuel economy.

\begin{tabular}{|c|c|c|c|c|c|c|}
\hline Cycle & Best Fuel Economy & Hybrid Configuration & C1, C2 & \% change from conventional & Engine Power & Motor Power \\
\hline & $\mathrm{mpg}$ & & & & $\mathrm{kW}$ & $\mathrm{kW}$ \\
\hline Freeway & 21.26 & Series & $0.5,2960$ & 41 & 65 \\
\hline CSHVC & 29.37 & Series & $0.1,1490$ & 126 & 32 \\
\hline Yard & 21.9 & Parallel & $0.3,1250$ & 68 & 71 \\
\hline Manhattan & 19.84 & Series & $0.2,3070$ & 53 & 14 \\
\hline Test D & 12.98 & Series & $0.2,1230$ & 0 & 21.4 \\
\hline Combined & 6.63 & Series & $0.2,1230$ & -49 & 48 & 72 \\
\hline
\end{tabular}

One of the limitations of this simulation method is evident in the Class $2 \mathrm{~B}$ results.

Since gear ratios are not included, no torque analysis can be done. This results in suggested configurations that are obviously in error. In Table 4.2.2, a parallel configuration with a $5 \mathrm{~kW}$ ICE and $12 \mathrm{~kW}$ electric motor is recommended. While this combination satisfies the power requirements over the Yard Cycle, an ICE more suited to use in a riding lawnmower would no be capable of producing the torque necessary to contribute to driving a nearly $4000 \mathrm{~kg}$ vehicle.

In all of the vehicles, the addition of auxiliary loads was extremely detrimental to the fuel economy. Generally, the fuel economy with auxiliary loads was half the fuel economy without auxiliary loads. This is due to requiring the engine to run inefficiently at low power levels throughout long periods of the cycles. When the vehicle is stopped without auxiliary loads, HEVs commonly allow the engine to shut off greatly reducing 
fuel economy over many cycles. The addition of auxiliary loads not only removes this option but also increases the fuel consumption during these periods.

Table 4.2.4 Class 6 optimum HEV without auxiliary load configurations and constants directly from simulation.

\begin{tabular}{|c|c|c|c|c|c|c|}
\hline Cycle & Best Fuel Economy & Hybrid Configuration & $\mathrm{C} 1, \mathrm{C} 2$ & \% change from conventional & Engine Power & Motor Power \\
\hline & $\mathrm{mpg}$ & & & & $\mathrm{kW}$ & $\mathrm{kW}$ \\
\hline Freeway & 10.79 & Series & $0.2,5240$ & 13 & 125 & 125 \\
\hline CSHVC & 11.3 & Series & $0.1,3200$ & 112 & 97 & 115 \\
\hline Yard & 9.61 & Series & $0.1,1030$ & 549 & 25 & 40 \\
\hline Manhattan & 6.61 & Parallel / Series & $0.2,2800$ & 113 & 64 & 125 \\
\hline Test D & 11.32 & Series & $0.4,2460$ & 37 & 102 & 125 \\
\hline Combined & 3.93 & Series & $0.4,2475$ & -39 & 120 & 125 \\
\hline
\end{tabular}

Table 4.2.5 Class 6 optimum HEV with auxiliary load configurations and constants directly from simulation.

\begin{tabular}{|c|c|c|c|c|c|c|}
\hline Cycle & Best Fuel Economy & Hybrid Configuration & $\mathrm{C} 1, \mathrm{C} 2$ & $\%$ change from conventional & Engine Power & Motor Power \\
\hline & $\mathrm{mpg}$ & & & & $\mathrm{kW}$ & $\mathrm{kW}$ \\
\hline Freeway & 8.35 & Parallel & $0.3,620$ & 26 & 56 & 100 \\
\hline CSHVC & 4.45 & Parallel & $0.2,1650$ & 77 & 78 & 78 \\
\hline Yard & 7.04 & Parallel & $0.1,2080$ & 1228 & 17 & 30 \\
\hline Manhattan & 2.95 & Parallel & $0.1,2025$ & 154 & 52 & 105 \\
\hline Test D & 4.44 & Parallel & $0.2,2420$ & 33 & 96 & 100 \\
\hline Combined & 1.98 & Parallel & $0.6,535$ & -38 & 58 & 110 \\
\hline
\end{tabular}

Results for the Class 6 vehicle appear to be more valid than those for the Class $2 \mathrm{~B}$ vehicle. The Yard Cycle results are still a fault because the cycle was designed specifically for Class 8 vehicles and the Class 6 conventional vehicle is overpowered. Torque analysis still appears to be a problem with the Yard Cycle with the suggested combination of a $17 \mathrm{~kW}$ ICE and a $30 \mathrm{~kW}$ electric motor. Interestingly, the HEV achieves poorer fuel economy on the Combined Cycle than the conventional vehicle. This is due to the ordering of the cycles, the control strategy, and the requirement for the vehicle to be perfectly charge sustaining over the cycle. Placing low power demand at the beginning of the cycle and ending it with more aggressive requirements places heavy demand on the batteries. To achieved charge sustaining operation, the ICE must be sized similarly to that used on a conventional vehicle and the control strategy requires a high $\mathrm{C}_{2}$ to sustain the SoC. The use of a large ICE means that it is forced to run at low power 
and poor efficiency during the early portion of the cycle resulting in poor fuel economy. As expected, only modest fuel economy improvement was seen on the Freeway Cycle due to the absence of large amounts of regenerative braking energy and the high average power demand.

Table 4.2.6 Class 8 optimum HEV without auxiliary load configurations and constants directly from simulation.

\begin{tabular}{|c|c|c|c|c|c|c|}
\hline Cycle & Best Fuel Economy & Hybrid Configuration & $\mathrm{C} 1, \mathrm{C} 2$ & $\%$ change from conventional & Engine Power & Motor Power \\
\hline & $\mathrm{mpg}$ & & & & $\mathrm{kW}$ & $\mathrm{kW}$ \\
\hline Freeway & 6.83 & Series & $0.2,6000$ & 20 & 205 & 205 \\
\hline CSHVC & 4.78 & Series & $0.1,3900$ & 52 & 190 & 205 \\
\hline Yard & 3.62 & Series & $0.1,3200$ & 289 & 65 & 102 \\
\hline Manhattan & 3.09 & Parallel & $0.3,2675$ & 63 & 146 & 135 \\
\hline Test D & 6.50 & Series & $0.5,1820$ & 39 & 182 & 205 \\
\hline Combined & 2.46 & Series & $0.5,1820$ & -35 & 201 & 205 \\
\hline
\end{tabular}

Table 4.2.7 Class 8 optimum HEV with auxiliary load configurations and constants directly from simulation.

\begin{tabular}{|c|c|c|c|c|c|c|}
\hline Cycle & Best Fuel Economy & Hybrid Configuration & $\mathrm{C} 1, \mathrm{C} 2$ & \% change from conventional & Engine Power & Motor Power \\
\hline & $\mathrm{mpg}$ & & & & $\mathrm{kW}$ & $\mathrm{kW}$ \\
\hline Freeway & 4.55 & Parallel & $0.5,600$ & 19 & 185 & 81 \\
\hline CSHVC & 1.95 & Parallel & $0.4,1240$ & 29 & 173 & 120 \\
\hline Yard & 1.46 & Parallel & $0.1,2080$ & 342 & 53 & 67 \\
\hline Manhattan & 1.16 & Parallel & $0.3,2000$ & 63 & 133 & 140 \\
\hline Test D & 2.49 & Parallel & $0.5,1825$ & 26 & 181 & 116 \\
\hline Combined & 1.14 & Parallel & $0.7,325$ & -40 & 185 & 75 \\
\hline
\end{tabular}

Since HEV buses that show significant fuel economy improvement are currently infiltrating the transit bus market, the results on the Manhattan Cycle are expected. Also, the small to moderate gains on the Freeway, CSHVC, and Test D Cycles where higher average speeds and power requirements dominate are expected. The problems evident in the analysis of the Classes $2 \mathrm{~B}$ and 6 conventional vehicle on the Yard Cycle are not present here. The cycle is designed for heavy-duty vehicles and specifically for Class 8 vehicles. Actual in-use yard spotter trucks are equipped with $175-200 \mathrm{~kW}$ engines which is approximately the same power used in the average Class 8 vehicle. For these reasons, it appears that the $300 \%$ fuel economy increase on the Yard Cycle is valid. 
Here, too, the HEV achieves poorer fuel economy than the conventional vehicle over the Combined Cycle for the same reasons as discussed with the Class 6 vehicle.

To further examine the fuel economy of HEVs over varied driving situations, the order of the cycles in the Combined Cycle were reversed and the vehicles were simulated again. A selection of these results is shown in Table 4.2.8.

Table 4.2.8 Simulation results from reversed Combined Cycle.

\begin{tabular}{|c|c|c|c|c|c|}
\cline { 2 - 6 } \multicolumn{1}{c|}{} & Conventional & HEV Configuration & C1, C2 & HEV Fuel Economy & \% change from conventional \\
\cline { 2 - 6 } \multicolumn{1}{c|}{} & & & & $\mathrm{mpg}$ & \\
\hline Class 2B & 13.00 & Series & $0.2,1400$ & 15.78 & 21 \\
\hline Class 6 & 9.61 & Series & $0.2,1700$ & 11.14 & 16 \\
\hline Class 8 & 3.86 & Series & $0.2,2900$ & 4.45 & 15 \\
\hline
\end{tabular}

This variation in fuel economy is due to the way the control strategy forces the vehicle to maintain charge-sustaining operation. In the original Combined Cycle, the high power requirement segments are near the end of the cycle forcing the engine to follow the road load closely throughout the cycle to avoid depleting the batteries. In the reversed cycle, the presence of the high power events near the beginning of the cycle allows the vehicle to recover SoC during the less demanding end portions through charging while driving. This phenomenon is not entirely realistic since requiring an HEV to return to the initial SoC after each use is not always possible.

\subsection{Road Grade Effects}

All federal test cycles assume that the vehicle is being operated on perfectly flat ground removing the effects of road grade. Obviously, vehicles are expected to operate on a wide variety of terrains and the effects of vehicle operation on hills or mountainous terrain on fuel economy and emissions is an important subject.

Figures 4.3.1 - 4.3.3 show the power requirement for each vehicle class to maintain constant speed on various grades determined from the road load equation (9). 
While the Class 2B vehicle can maintain highway speed on a $7 \%$ grade without exceeding its maximum power, the Class 6 vehicle would be limited to $30 \mathrm{mph}$ and the Class 8 could maintain $20 \mathrm{mph}$.

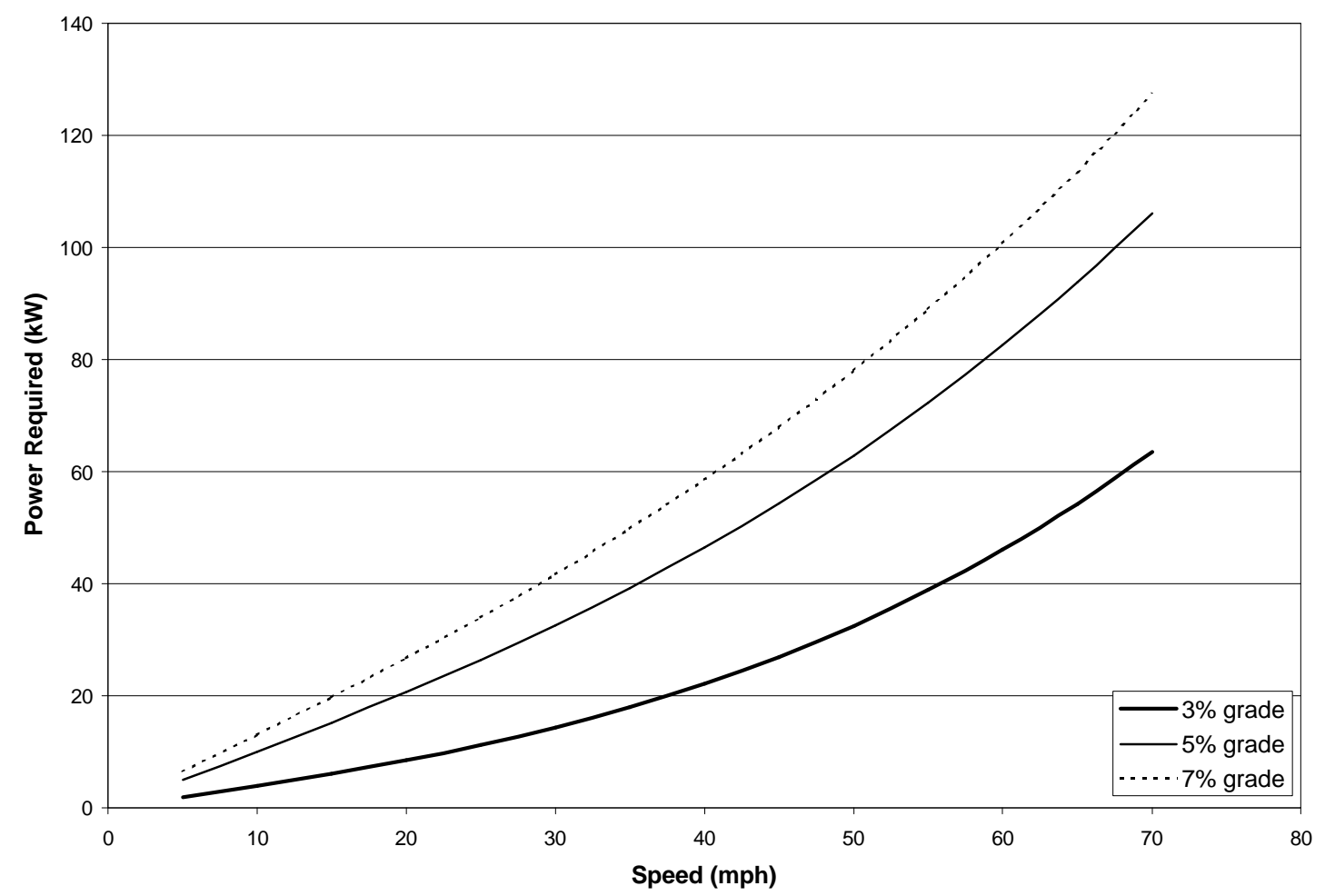

Figure 4.3.1 Power required to maintain constant speed on various grades for Class 2B. 


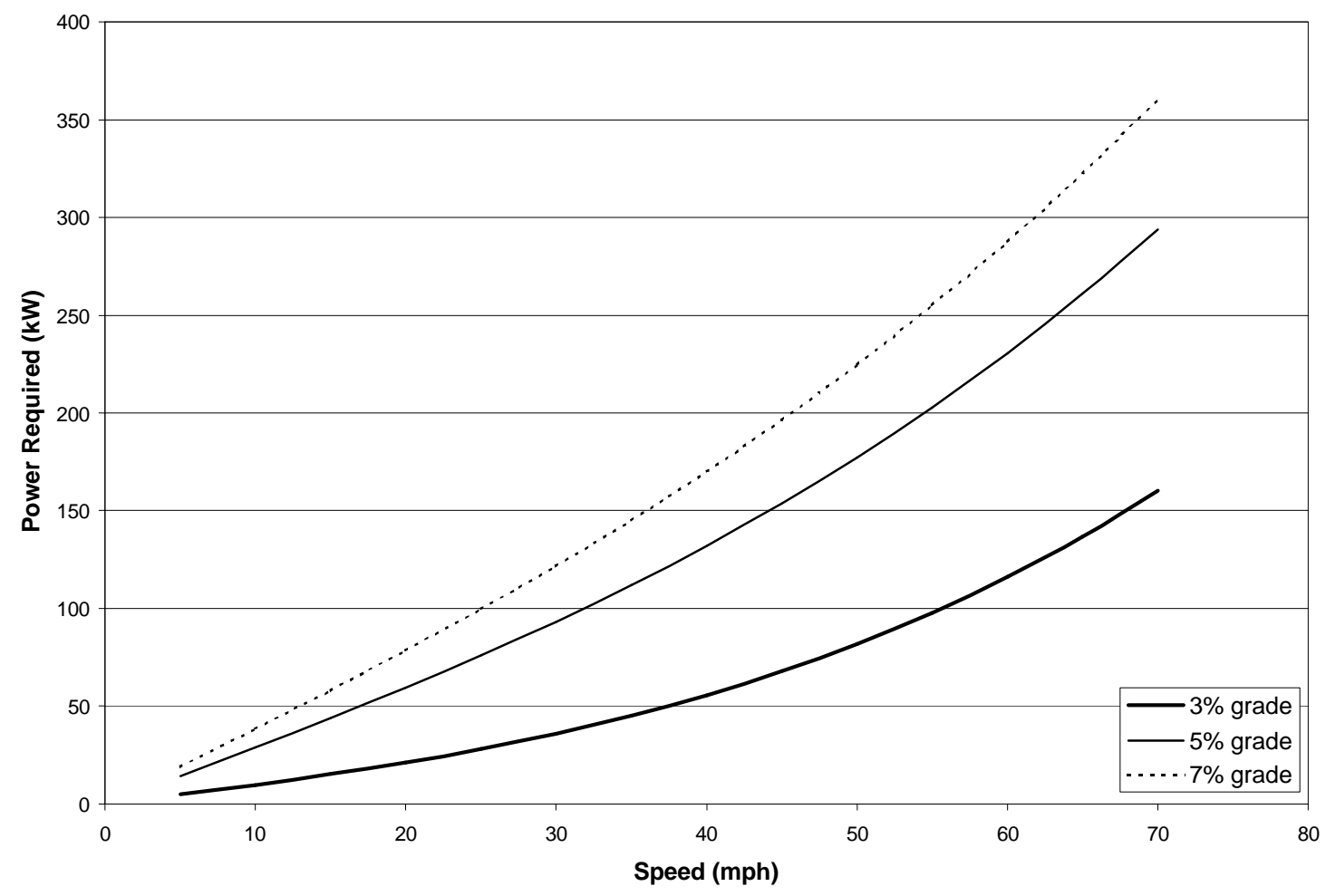

Figure 4.3.2 Power required to maintain constant speed on various grades for Class 2B.

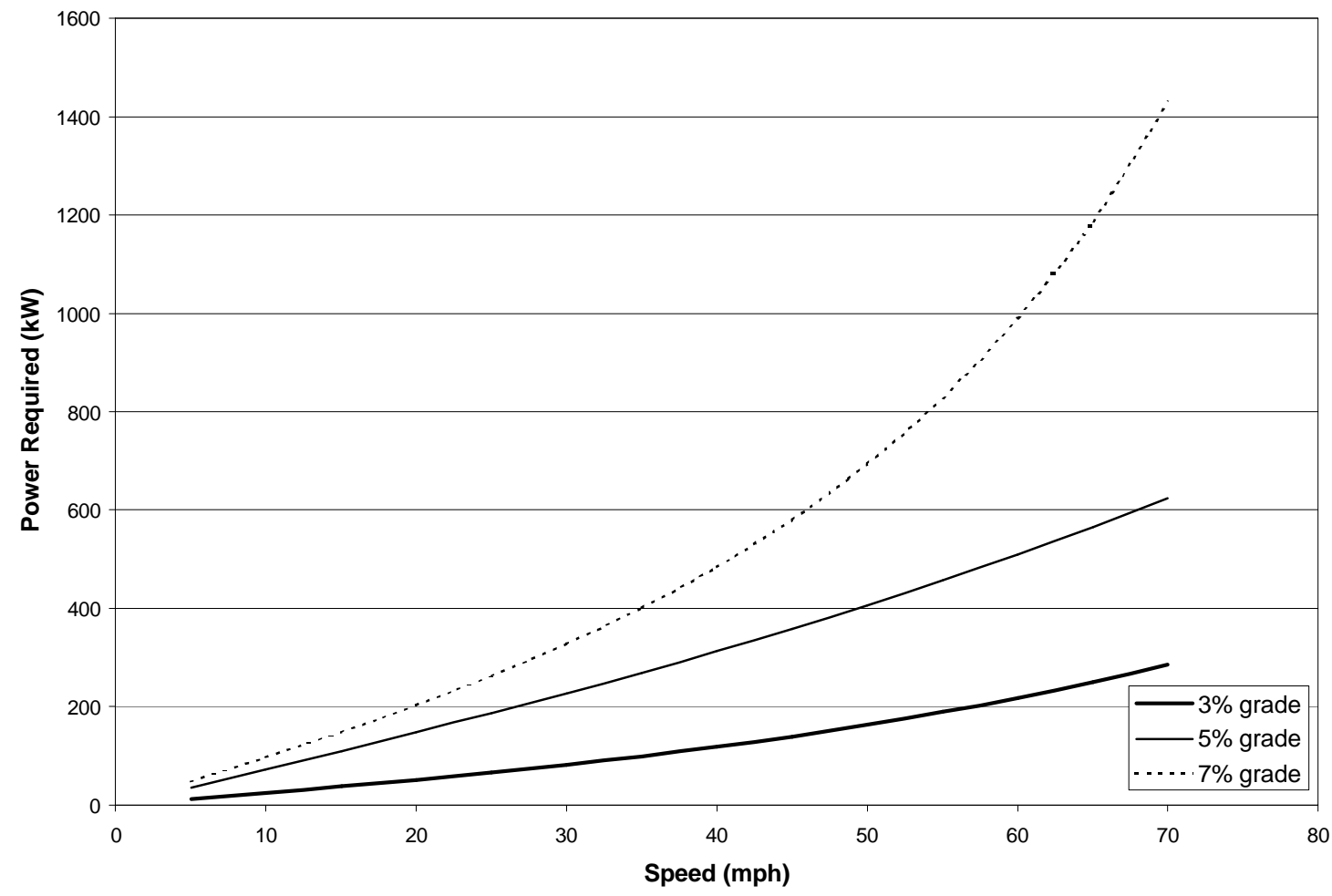

Figure 4.3.3 Power required to maintain constant speed on various grades for Class 8. 
Figure 4.3.4 shows the effect of superimposing a sinusoidal terrain with a maximum $2 \%$ grade on the Freeway Cycle. When adding road grade to a cycle, the phase of the road grade is very important. Here, the varying terrain results in very little change in the positive and negative power peaks, but shifting it slightly would cause a dramatic increase in the magnitude of the peaks. This demonstrates one of the problems with analyzing the effects of road grade. Real terrain is not perfectly sinusoidal and is difficult to integrate with existing cycles. In the Figure 4.3.4, a series HEV shows a 16\% increase in fuel economy over a conventional vehicle, $3 \%$ higher than the increase when road grade is not considered, but these results are valid only for this specific trace. Any change in the phase of the terrain would dramatically affect the fuel economy.

The stop and start points of a cycle including road grade are very important. If the vehicle begins at the bottom of a hill and stops at the top, it has no chance of being charge sustaining and is not allowed to take advantage of the regenerative braking energy available while descending the hill. Similarly, if a cycle begins at the top of a hill and ends at the bottom, the power requirements over the cycle will be artificially low and a vehicle designed specifically to meet these requirements would be totally unsuited to climbing the same hill. 


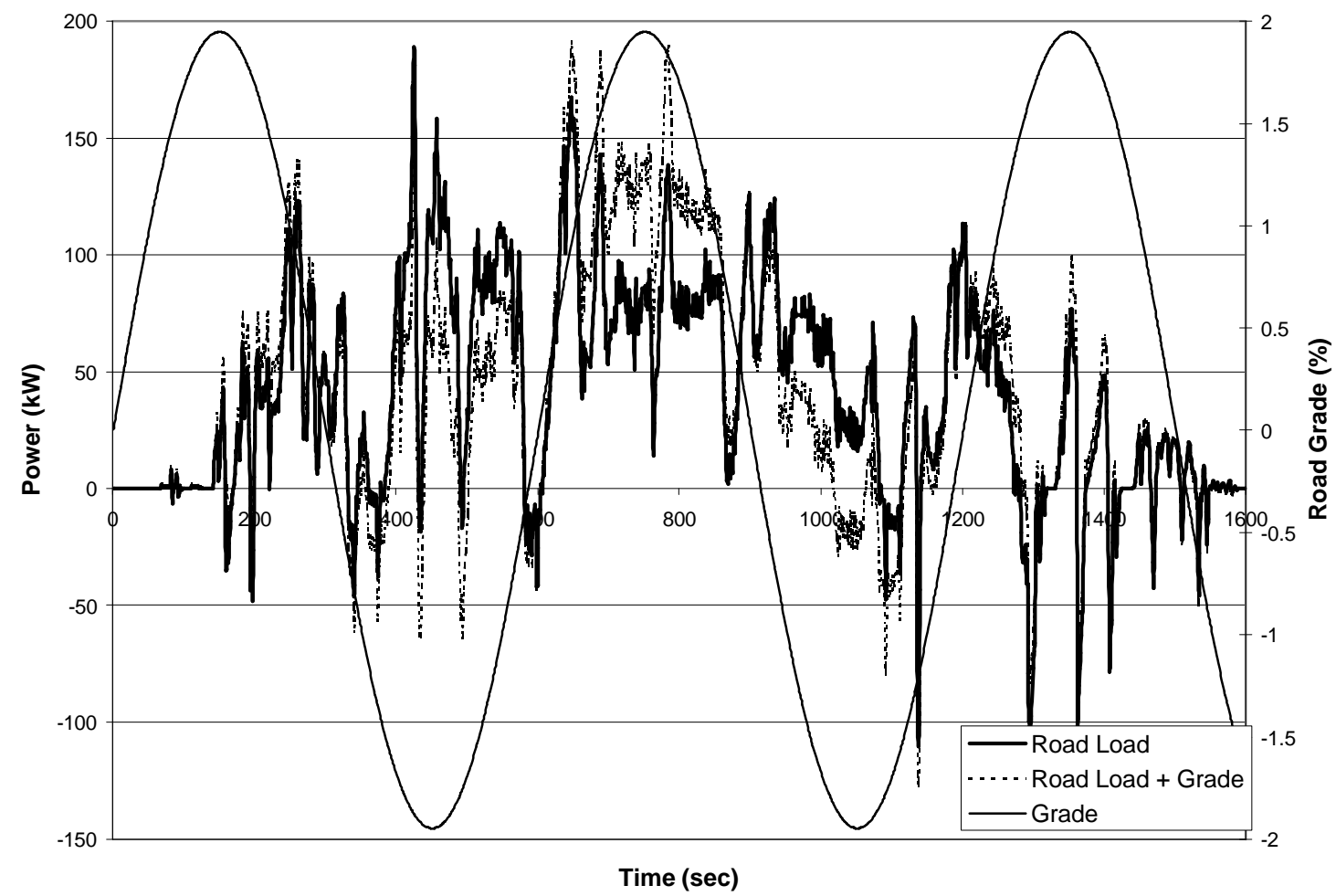

Figure 4.3.4 Power required for Class 6 vehicle over Freeway Cycle with and without road grade.

\subsection{Niche Markets}

A niche market for any vehicle is a specialized area of vehicle operation that allows the vehicles to be specifically tailored to that type of operation. Niche markets are especially suited to the application of HEV technology. Since HEVs can be extremely specialized through selection of components and control strategy, they can be heavily optimized for specific operations. A few of these include transit buses, yard spotters, and ‘cubage' limited vehicles.

\subsubsection{Transit Buses}

Manufacturers of hybrid electric vehicles are already exploiting the transit bus niche market. The highly transient nature of their operation results in the availability of large amounts of regenerative braking energy. Moderate dynamic requirements, and low 
average power and speed make this market especially suited to series HEVs. Since the buses are never expected to leave their regular operating range, they can be specifically designed for urban operation without fear of making them unsuitable for other operation.

\subsubsection{Yard Spotters}

Yard spotters are another niche market that has been somewhat exploited by conventional vehicle manufacturers but has yet to attract interest from $\mathrm{HEV}$ manufacturers. Yard spotting consists of moving empty or loaded trailers from position to position either for storage, loading, or pickup. As seen in the Yard Cycle, developed from these types of operations, yard trucks operate a low speed and power making them suitable for HEV application. The low acceleration demands would minimize the effects of the battery, motor, and controller 'dead weight' on the vehicle's fuel economy. As with the transit buses, yard spotters are never expected to leave their home operating area allowing them to be very specialized. The low average power requirement would make these vehicles especially suited to a series HEV drivetrain with a very small ICE being used as a generator. The dynamic performance of such an HEV should be superior to a conventional vehicle due to the speed - torque characteristics of electric motors providing maximum torque at low speed.

\subsubsection{Cubage Limited Vehicles}

Hybrid electric powertrains are quite heavy. In the simulated Class 8 vehicle, the batteries along with the battery box and electronics, electric motor, and controller weigh $500 \mathrm{~kg}$. While this is only $1.5 \%$ of the GVW of the average Class 8 vehicle, commercial vehicles operate on very small profit margins and, if the vehicle's payload is limited by the GVW, the weight of the payload, hence the profit, is decreased by the weight of the 
hybrid powertrain. Many vehicles are not loaded to the GVW during operation. For example, a truck hauling bread will be limited by the available volume rather than weight. In this case, the weight penalty from of the hybrid powertrain will be based on the inertial penalty in the road load equation but will not cut into the profit margin of the operator and the benefits of improved fuel economy become more substantial. 


\section{Conclusions}

If used correctly, hybrid electric technology has the potential to significantly increase the fuel economy of heavy-duty vehicles. This is especially true in niche markets where the advantages of HEV drivetrains can be maximized through specialization. A potential 300\% increase in fuel economy for Class $8 \mathrm{HEV}$ yard spotters is a particularly interesting. Cubage limited vehicles also show the potential for a highly profitable HEV market. Incorrectly applied hybrid powertrains can also lead to poorer fuel economy than in conventional vehicles as shown in the $40 \%$ decrease in fuel economy for a Class $8 \mathrm{HEV}$ over the Combined Cycle.

The dependence of HEV fuel economy on the order of events in a cycle was shown to be very significant by reversing the order of the cycles in the Combined Cycle. The Class 6 vehicle went from a 39\% decrease in fuel economy on the Combined Cycle to a $16 \%$ increase on the reversed cycle.

Removing auxiliary loads from the engines on HEVs and even conventional vehicles, where possible, by making them electrically driven would greatly improve fuel economy. Not only would the load on the engine be decreased, but also the overall engine efficiency over a driving cycle would be increased.

Further refinement of the Excel simulation would only increase the reliability of the results, but, as features such as gear ratios, shifts, engine and motor maps, and torque analysis are included, the opportunity for user error and for producing highly subjective results is increased. 
Further investigation might include an overview of the process of selecting an appropriate HEV for a given application. A potential HEV customer would be expected to analyze the vehicles in his or her fleet examining both the vehicles and their typical operational modes. After an optimum configuration and component sizes were found, the fuel economy and additional vehicle cost of the resulting HEV could be compared to the fuel economy and cost of a conventional vehicle to determine the potential savings. If these savings were significant, the customer would be advised to purchase and operate HEVs. 


\section{References}

[1] http://www.ta.doc.gov/pngv/ (4/29/2001)

[2] http://www.osti.gov/hvt/21stcenturytruck.pdf (5/2/2001)

[3] Tóth-Nagy, Csaba, "Investigation and Simulation of the Planetary Combination Hybrid Electric Vehicle”, Masters Thesis, West Virginia University, 2000.

[4] Parsley, William, "New York City Transit Operating Experience with Hybrid Transit Buses", SAE / NESEA Hybrid Electric Vehicles in the Bus and Truck Markets TOPTEC: New Ways of Building Better Heavy Duty Vehicles, May 11-12, 2000.

[5] An, F., Stodolsky, F., Vyas, A., Cuenca, R., "Scenario Analysis of Hybrid Class 3

- 7 Heavy Vehicles", SAE Paper 2000-01-0989.

[6] http://www.dieselnet.com

[7] Wang, M., Vehicle Greenhouse Gas Emissions: The Role of Fuel-Cycle Analysis, FutureTruck 2000 Workshop, Pontiac, MI, Sept. 20, 1999.

[8] http://www.gmc.com/

[9] http://www.4adodge.com/

[10] http://www.fordvehicles.com/common/home2.asp

[11] West Virginia University Transportable Heavy-Duty Emissions Testing Laboratory Database, Mechanical and Aerospace Engineering Dept., College of Engineering and Mineral Resources, West Virginia University, January 2001.

[12] Clark, N. N., Daley, J. J., Nine, R. D., Atkinson, C. M., “Application of the New City-Suburban Heavy Vehicle Route (CSHVR) to Truck Emissions 
Characterization", SAE Fuels and Lubricants Meeting, SAE Paper 1999-01-1467, Dearborn, MI, May 3-6, 1999.

[13] Clark, N. N. , McKain, D. L., Balon, T. H., Moynihan, P. J., Lynch, S. A., Webb, T. C., "Characterization of Emissions from Hybrid-Electric and Conventional Buses", SAE Fuels and Lubricants Meeting, SAE Paper 2000-01-2011, Paris, France, June 19-22, 2000.

[14] Nine, R. D., Clark, N. N., Daley, J. J., Atkinson, C. M., "Development of a Heavy-Duty Chassis Dynamometer Driving Route", Department of Mechanical and Aerospace Engineering, West Virginia University, Morgantown, WV, 1999.

[15] Gillespie, T. D., Fundamentals of Vehicle Dynamics, SAE, Warrendale, PA, 1992.

[16] http://www.hepi.com/

[17] Kellermeyer, W. F., "Development and Validation of a Modular Hybrid Electric Vehicle Simulation Model", Masters Thesis, West Virginia University, 1998.

[18] Davis, S. C., "Transportation Energy Data Book: Edition 19", Oak Ridge National Laboratory, Oak Ridge, TN, September 1999.

[19] "Hybrid-Electric Drive Heavy-Duty Vehicle Testing Project - Final Emissions Report", NAVC, DARPA, Northeast Advanced Vehicle Consortium, M. J. Bradley \& Associates, Inc., and West Virginia University, 2000. 


\section{Appendix}




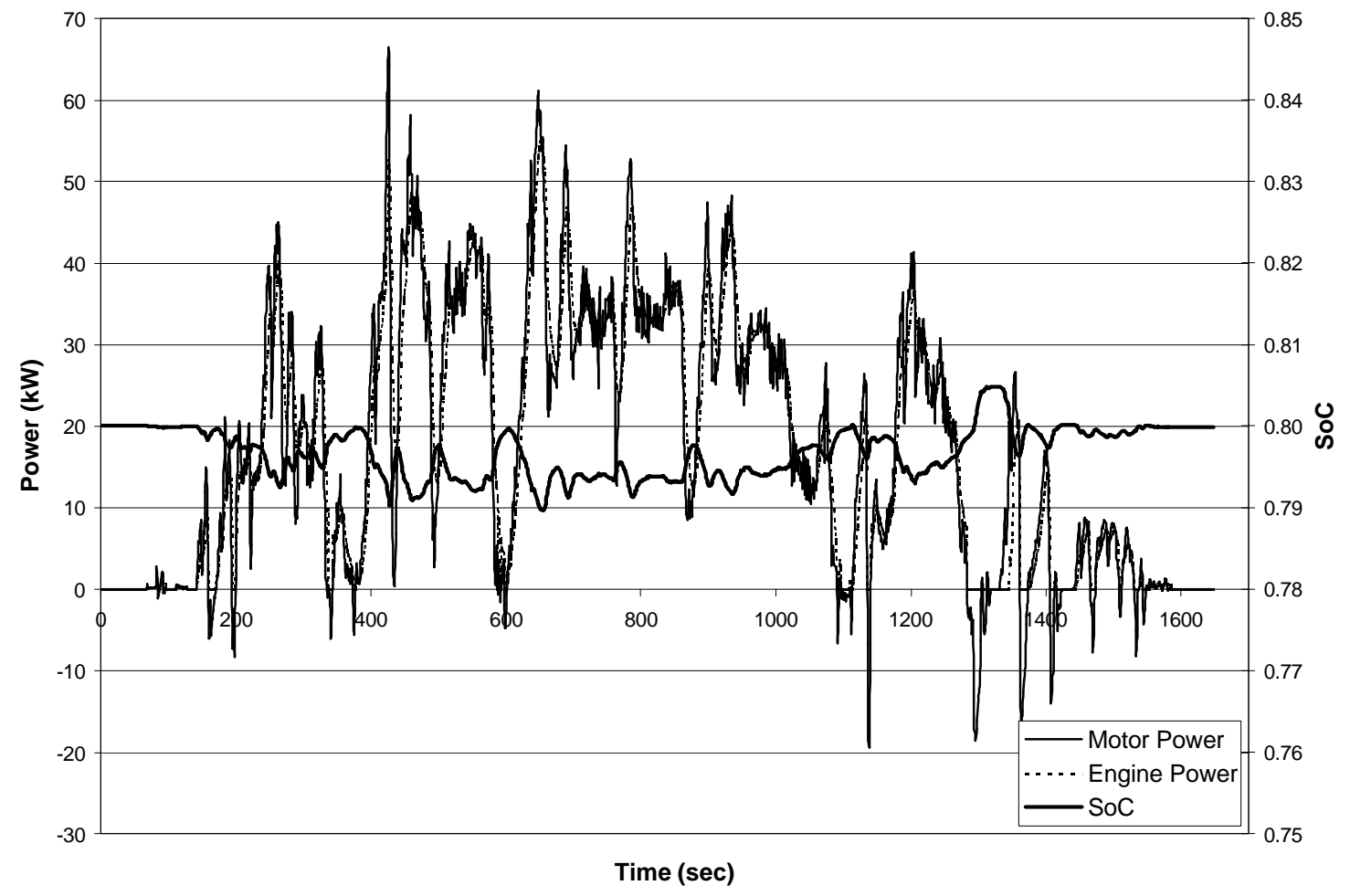

Figure A.1 Class 2B Series HEV on Freeway Cycle without auxiliary load.

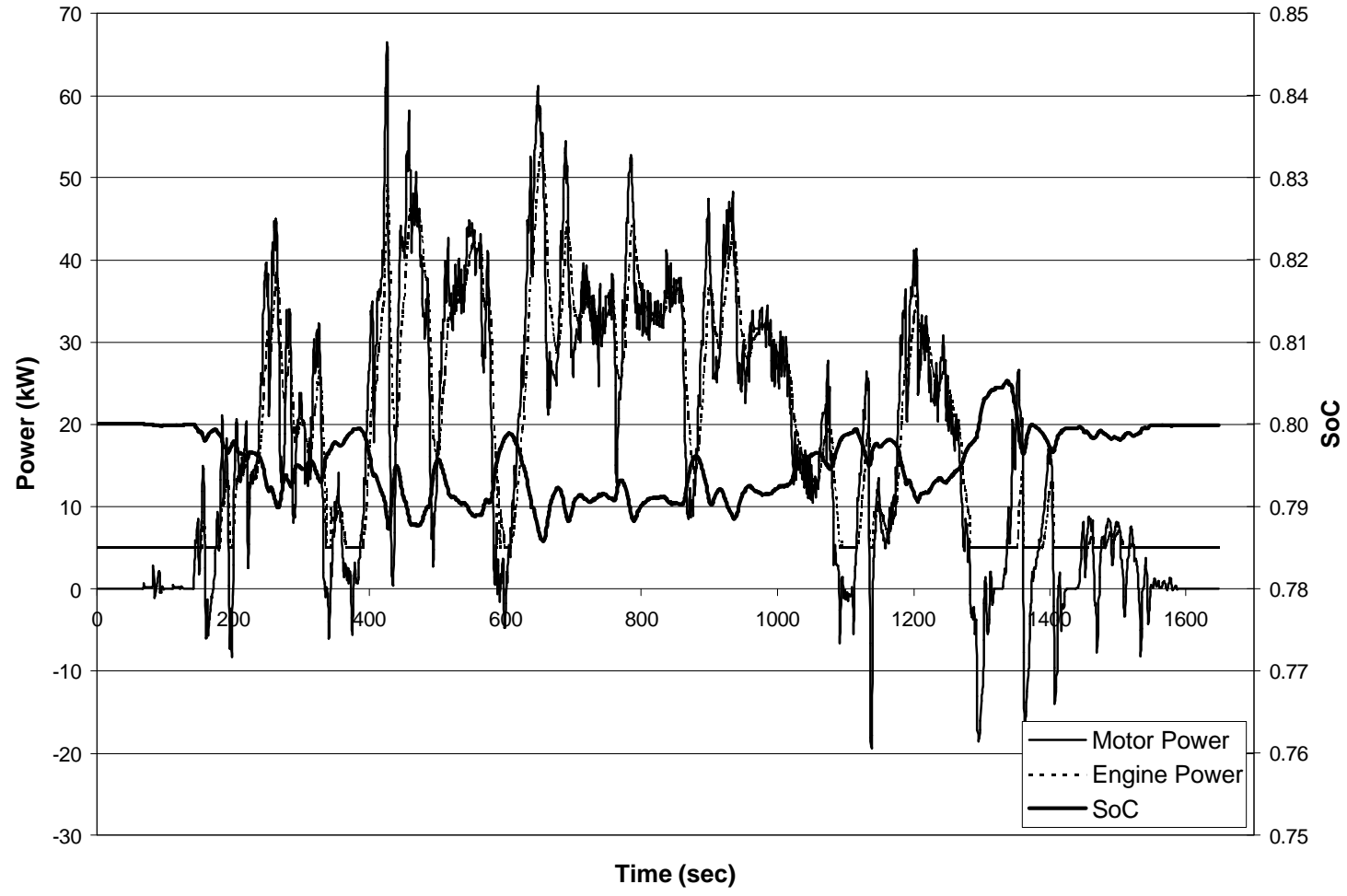

Figure A.2 Class 2B Series HEV on Freeway Cycle with auxiliary load. 


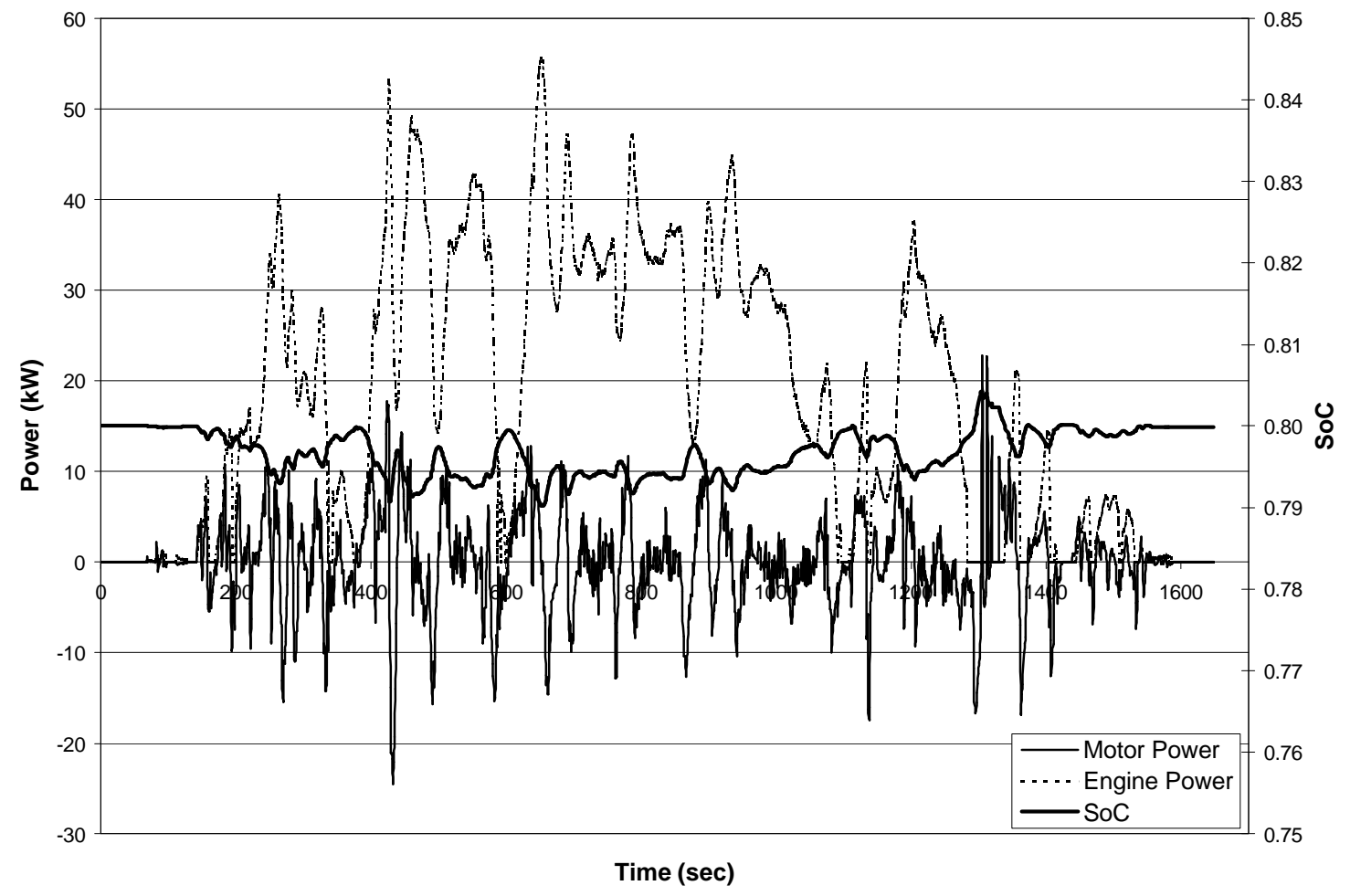

Figure A.3 Class 2B Parallel HEV on Freeway Cycle without auxiliary load.

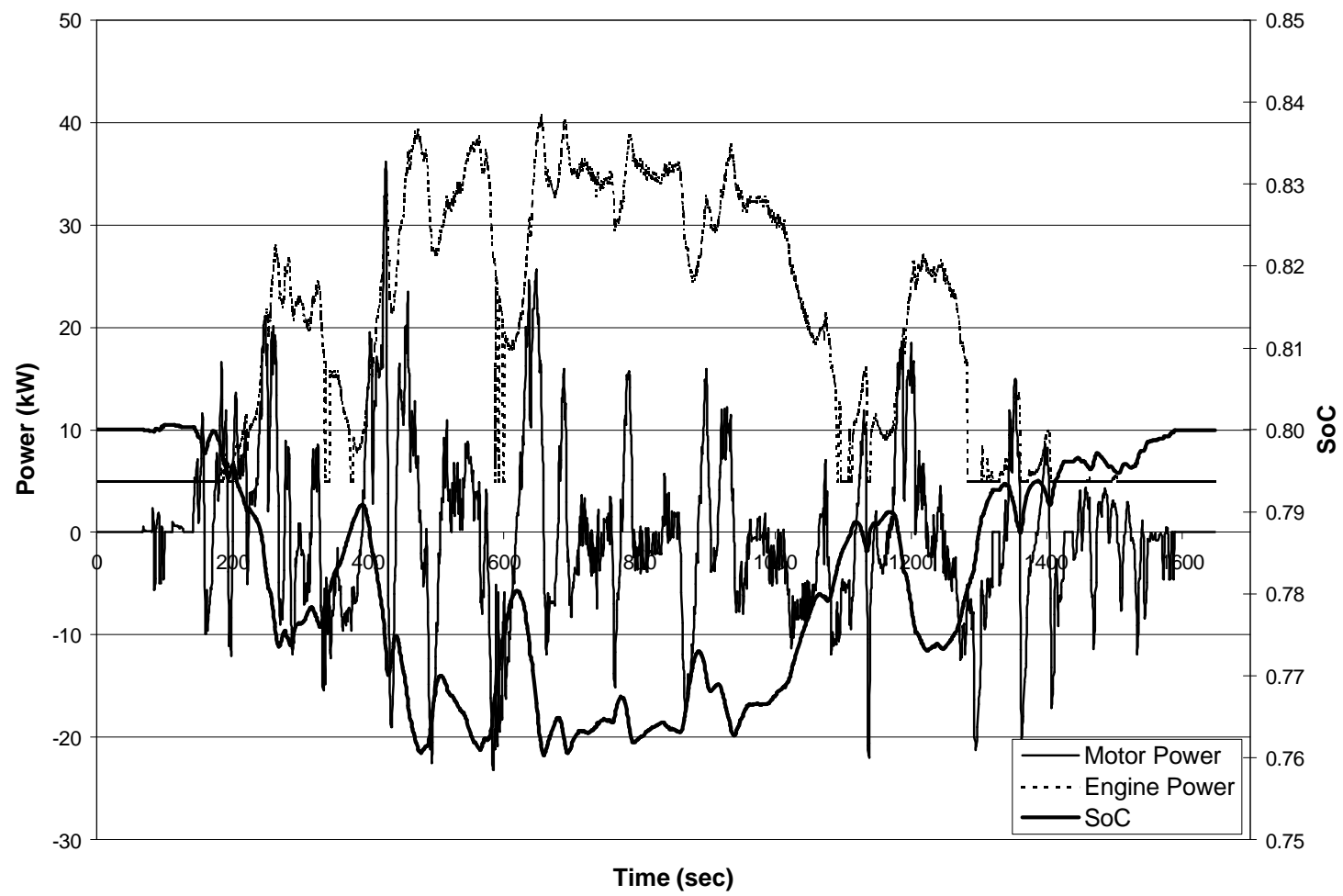

Figure A.4 Class 2B Parallel HEV on Freeway Cycle with auxiliary load. 


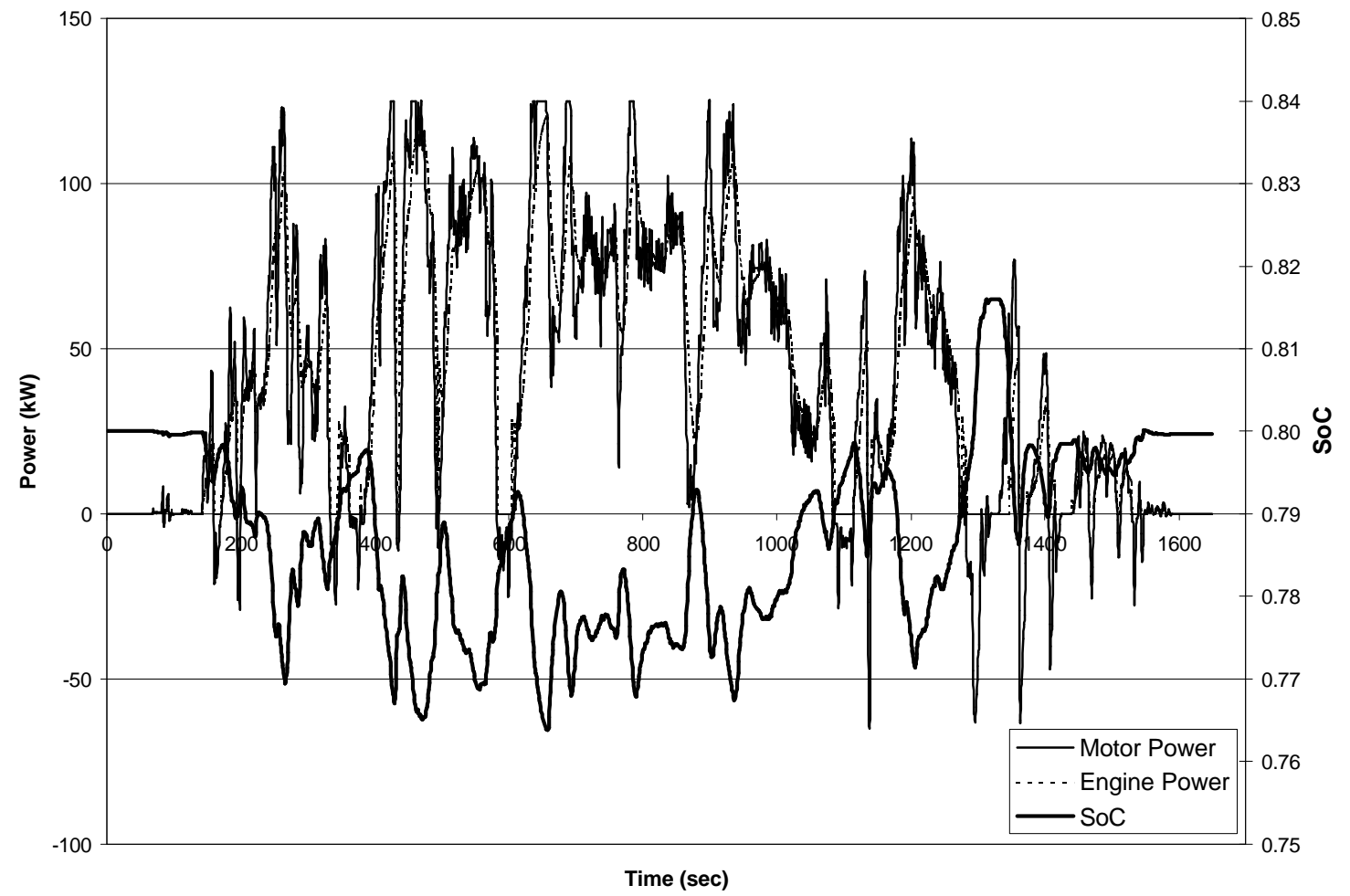

Figure A.5 Class 6 Series HEV on Freeway cycle without auxiliary load.

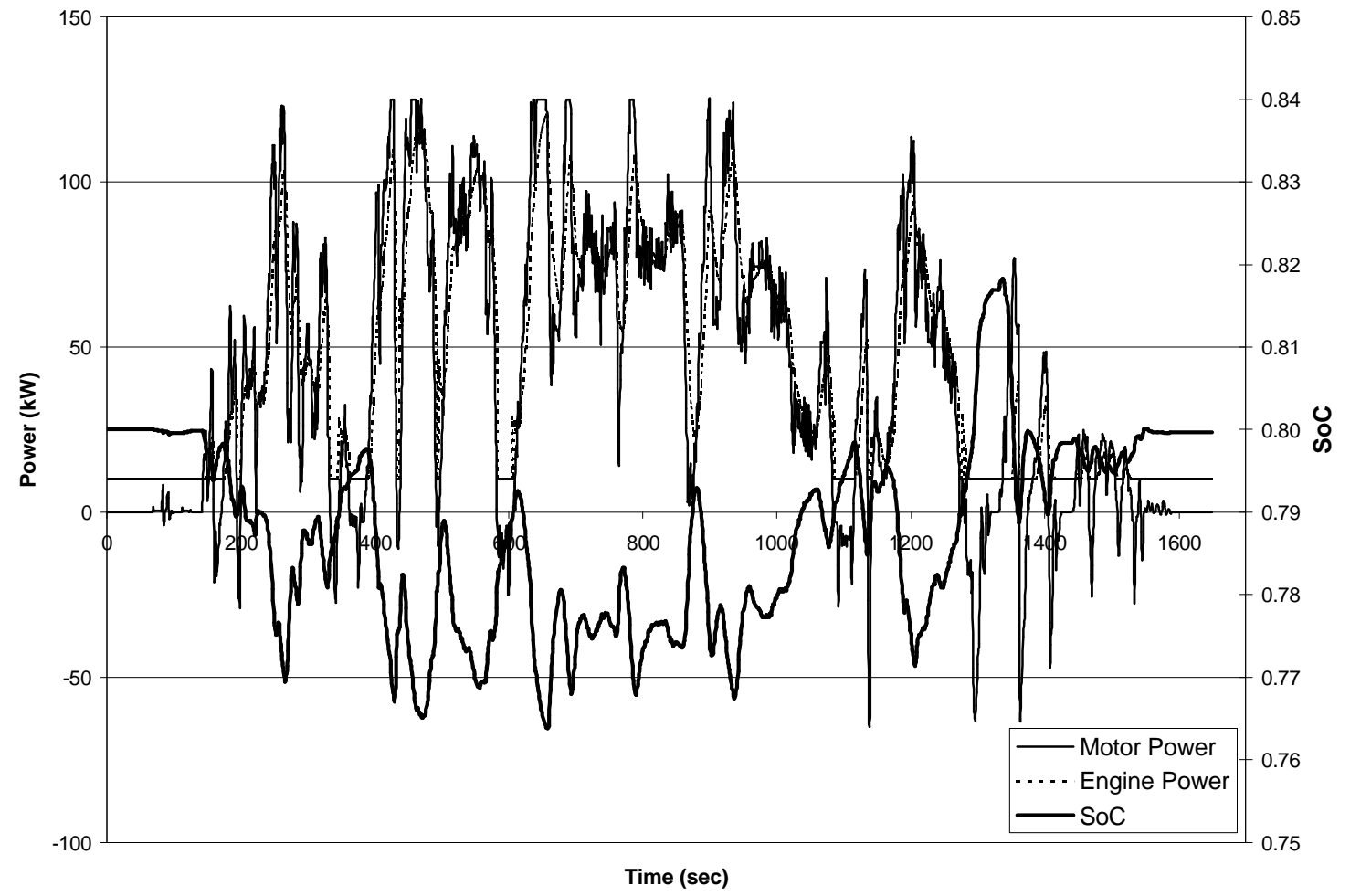

Figure A.6 Class 6 Series HEV on Freeway Cycle with auxiliary load. 


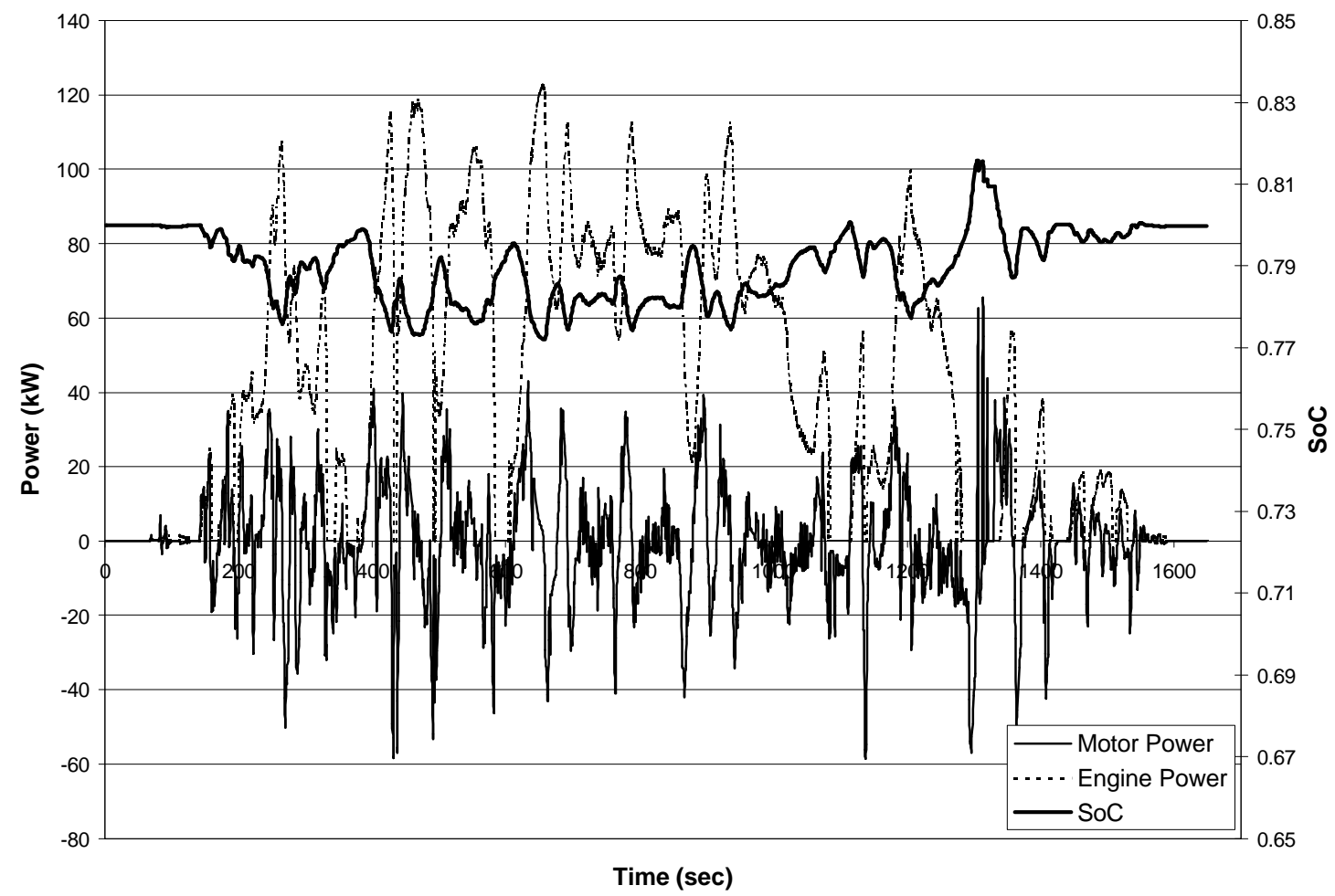

Figure A.7 Class 6 Parallel HEV on Freeway Cycle without auxiliary load.

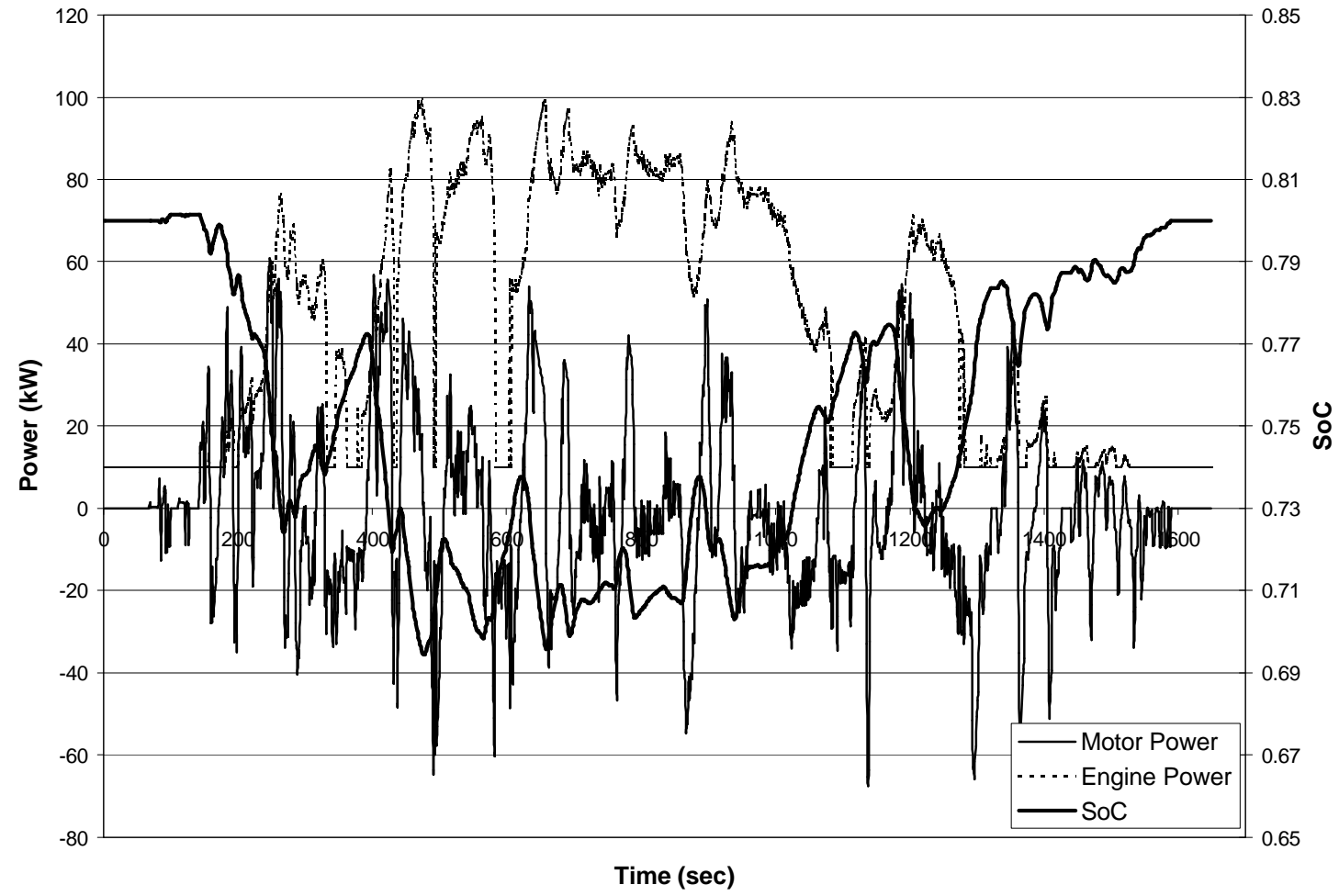

Figure A.8 Class 6 Parallel HEV on Freeway Cycle with auxiliary load. 


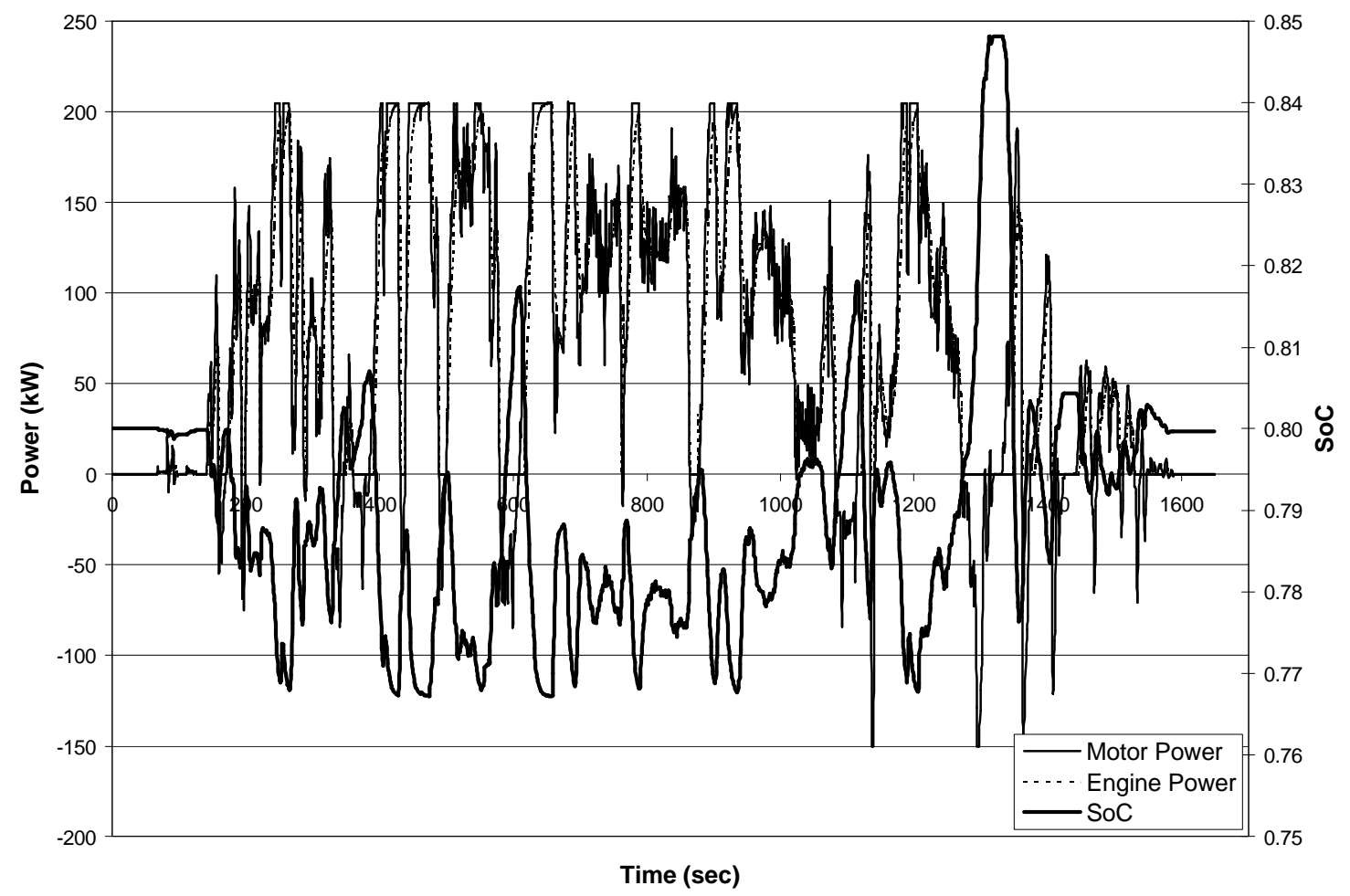

Figure A.9 Class 8 Series HEV on Freeway Cycle without auxiliary load.

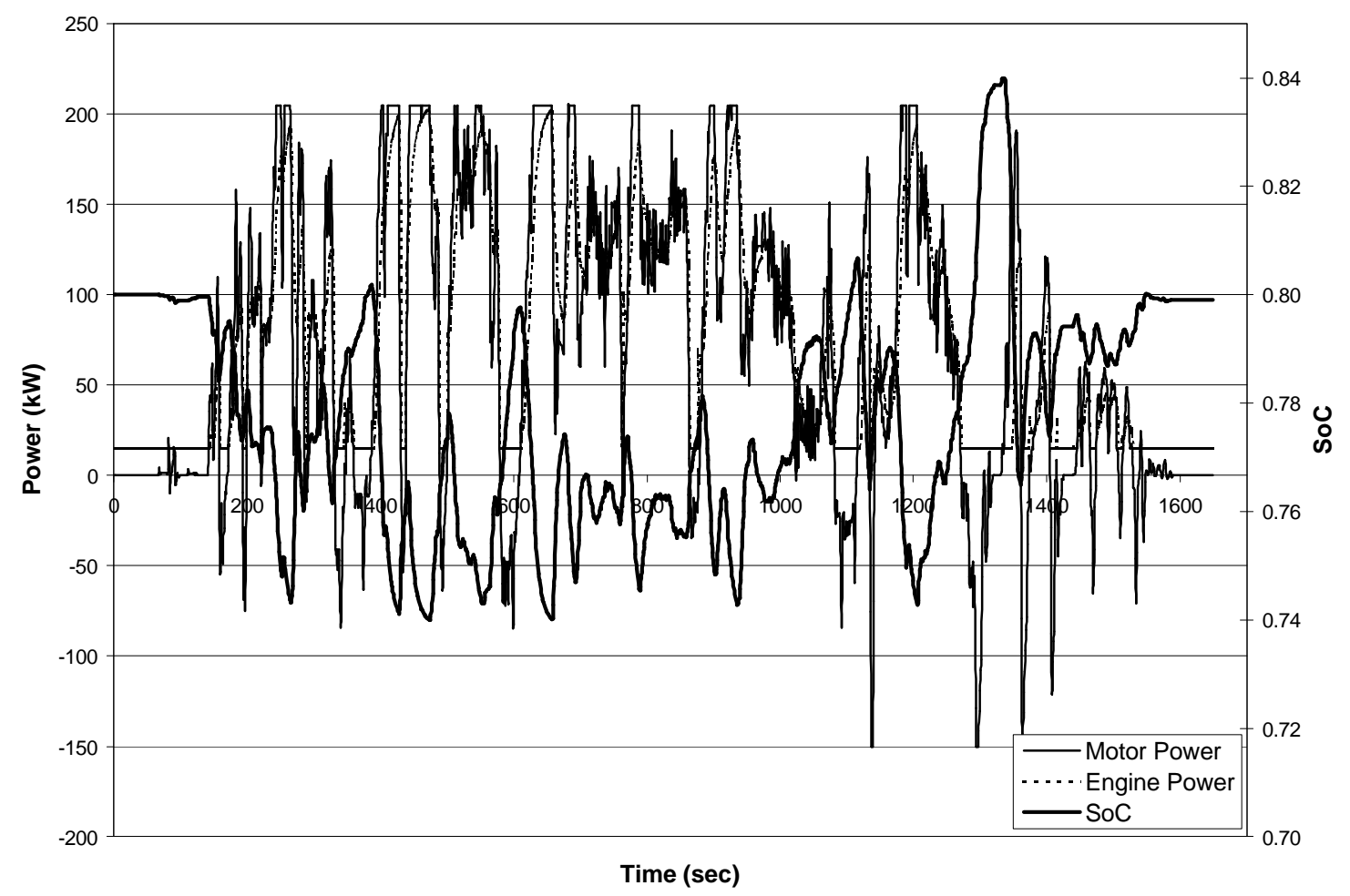

Figure A.10 Class 8 Series HEV on Freeway Cycle with auxiliary load. 


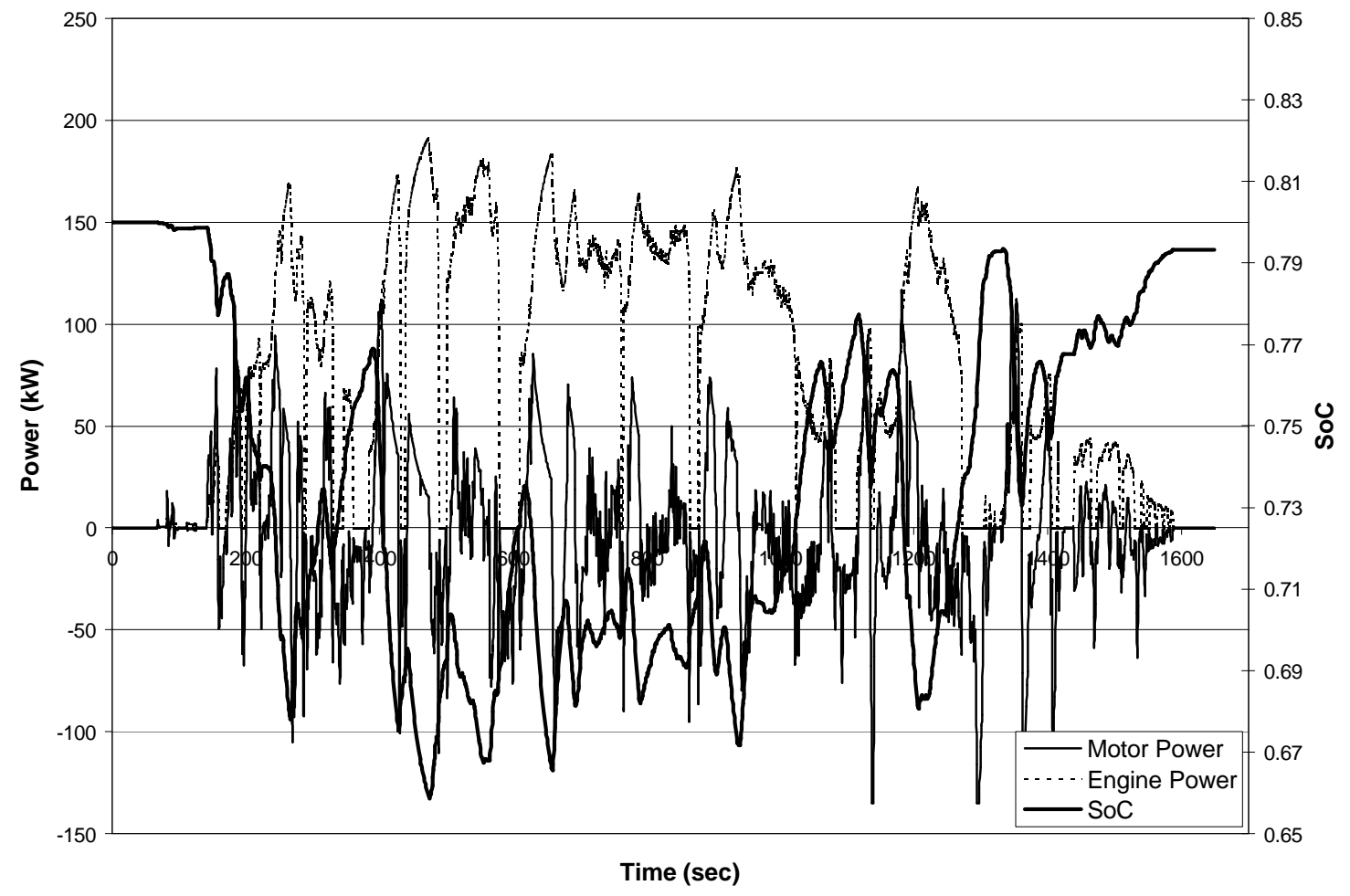

Figure A.11 Class 8 Parallel HEV on Freeway Cycle without auxiliary load.

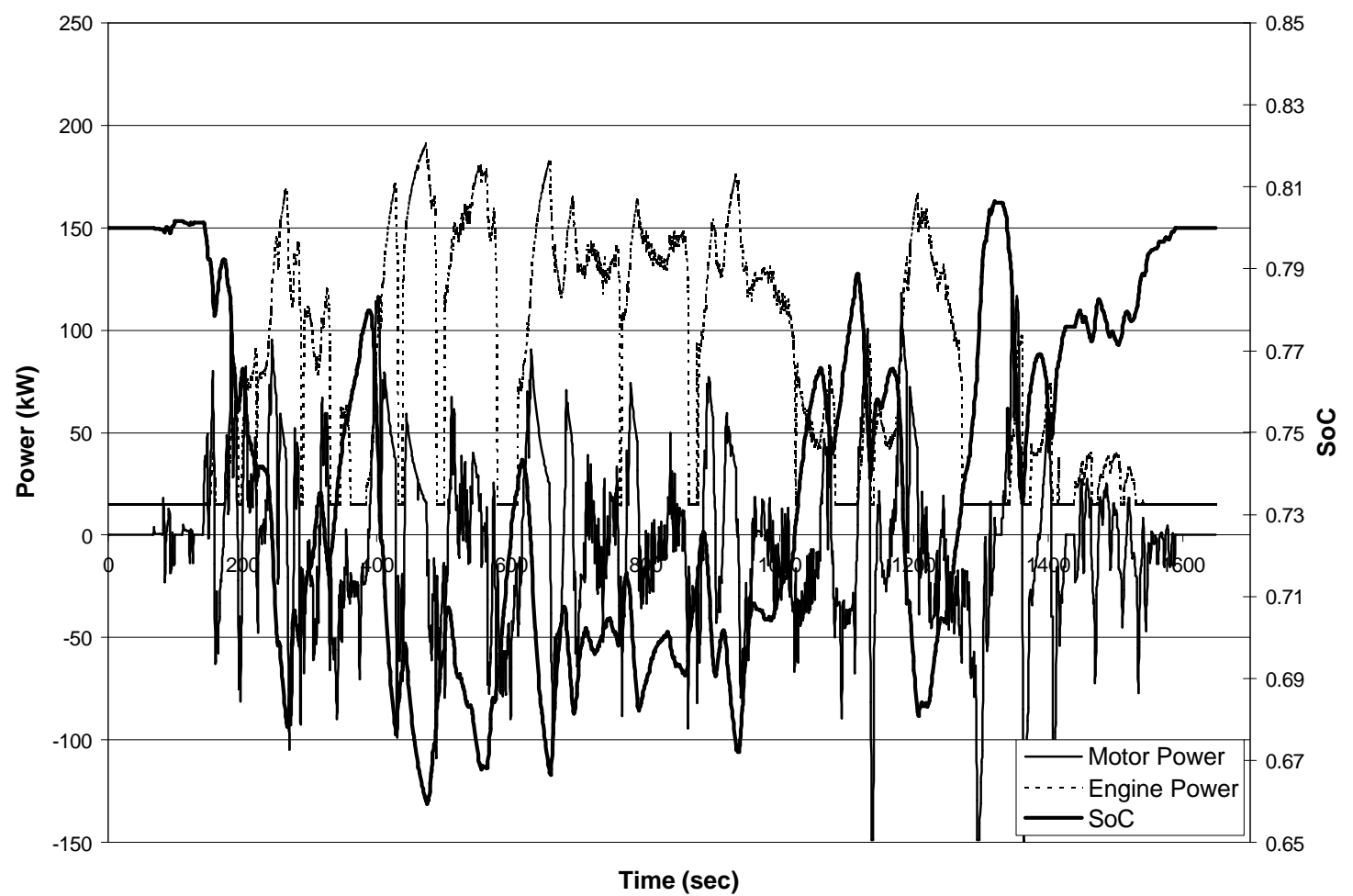

Figure A.12 Class 8 Parallel HEV on Freeway Cycle with auxiliary load. 


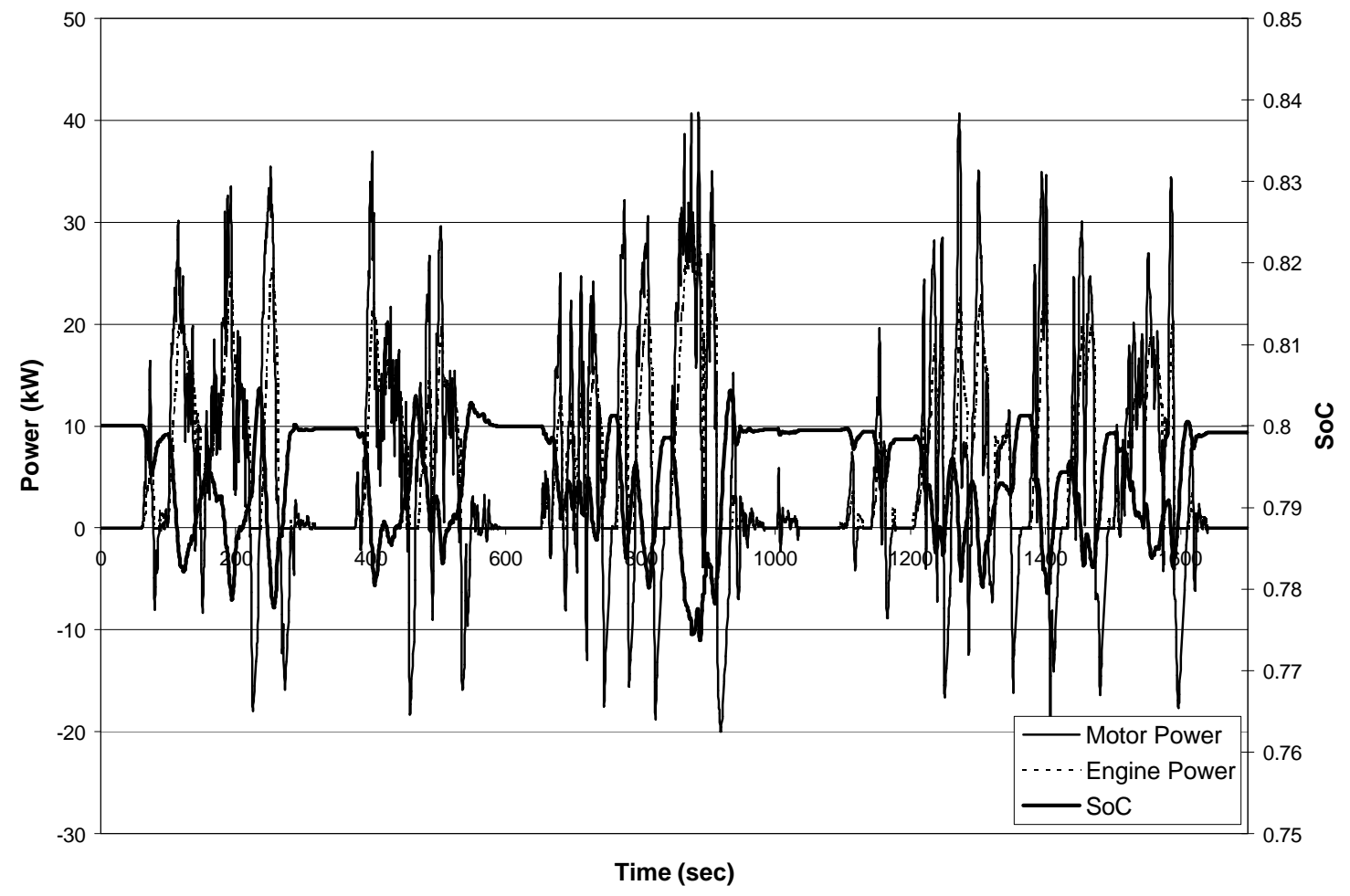

Figure A.13 Class 2B Series HEV on CSHVC without auxiliary load.

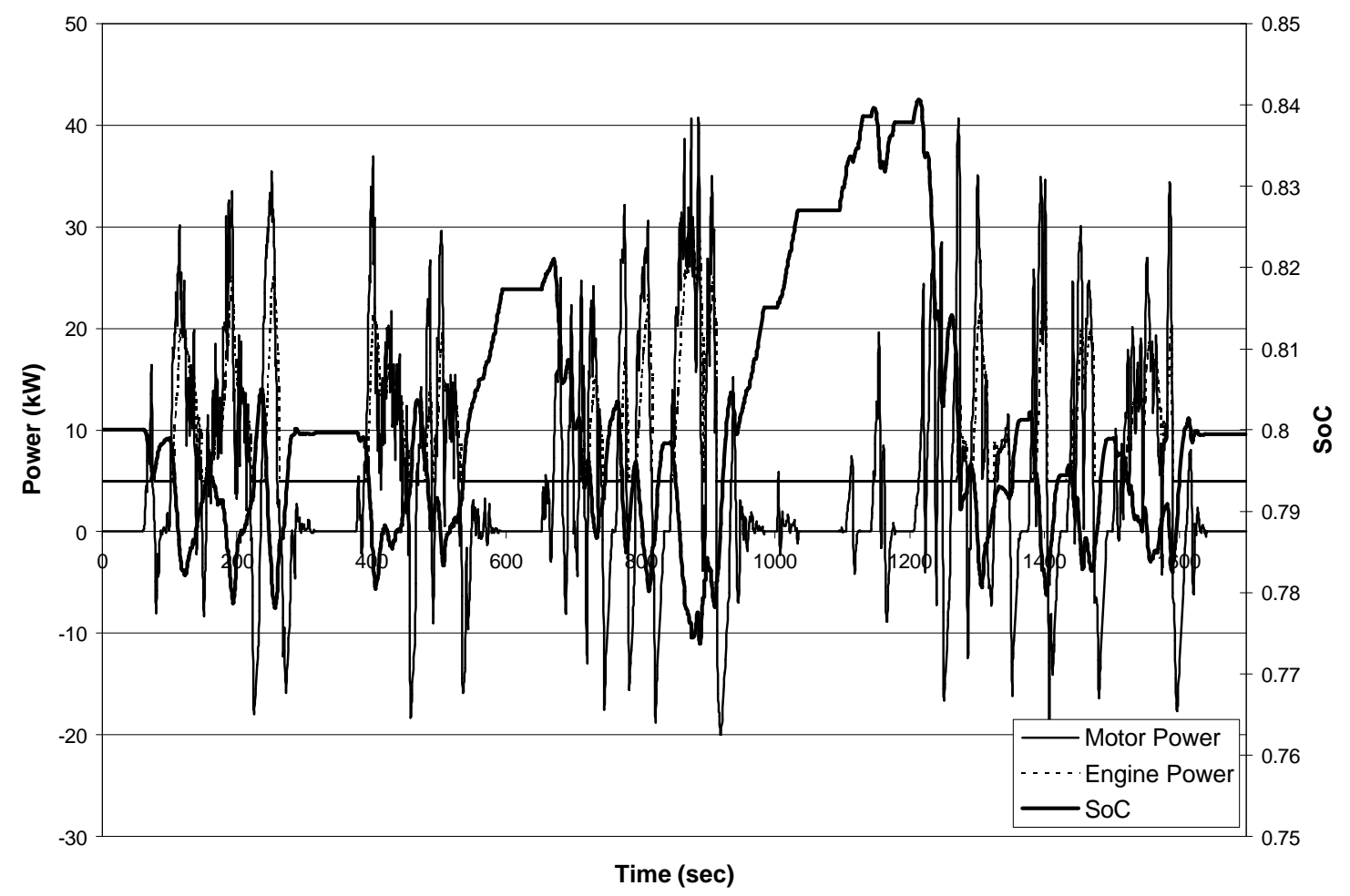

Figure A.14 Class 2B Series HEV on CSHVC with auxiliary load. 


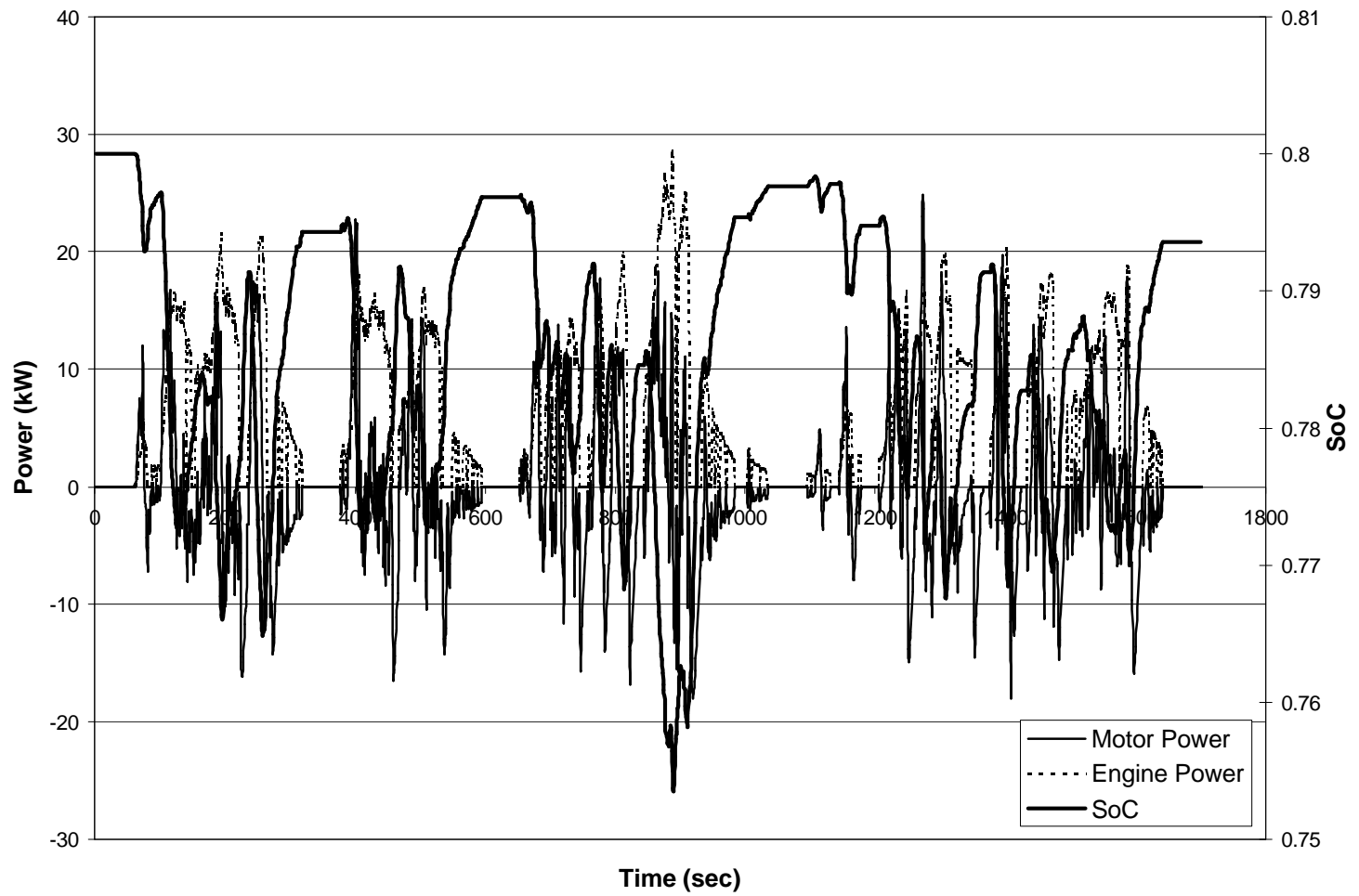

Figure A.15 Class 2B Parallel HEV on CSHVC without auxiliary load.

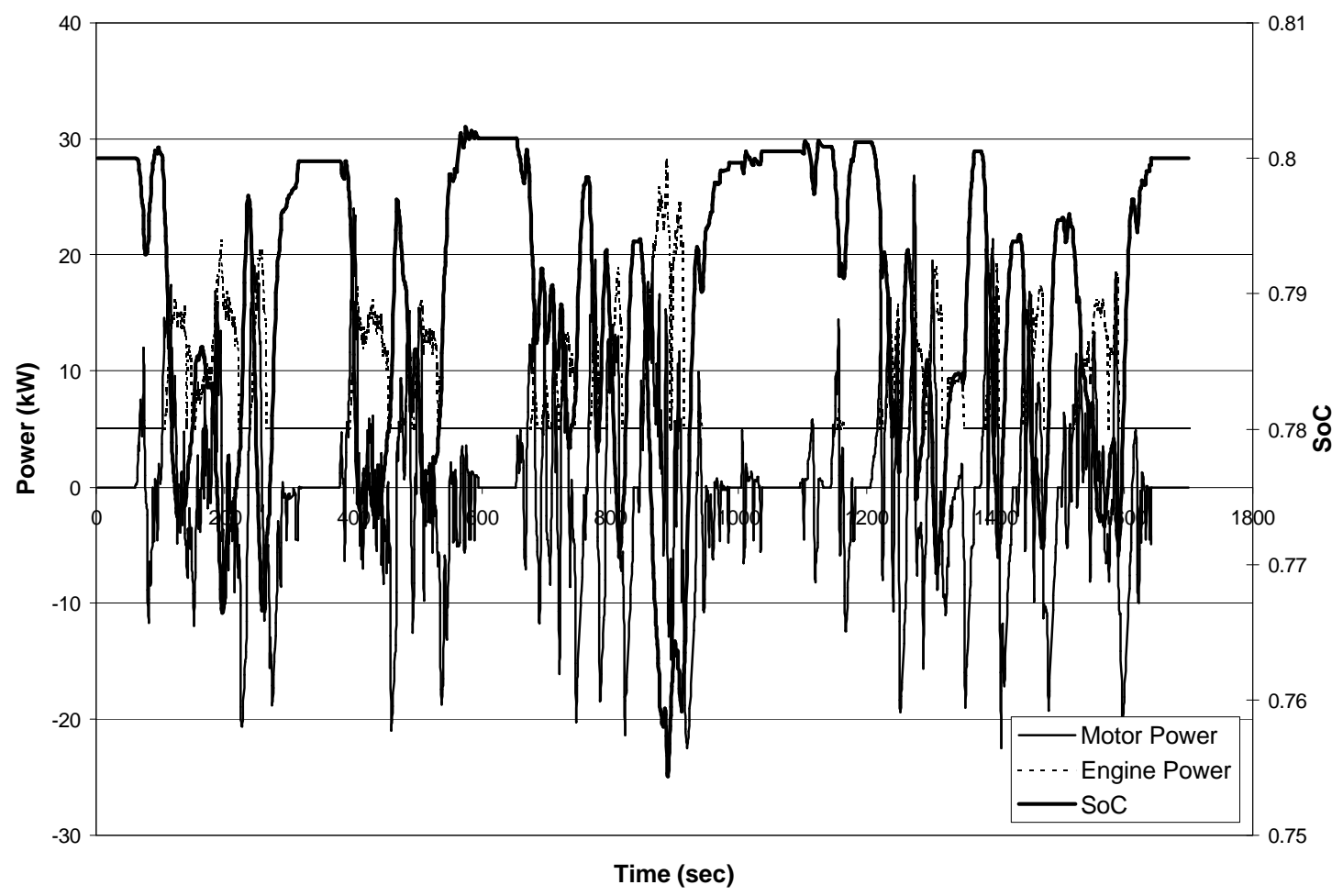

Figure A.16 Class 2B Parallel HEV on CSHVC with auxiliary load. 


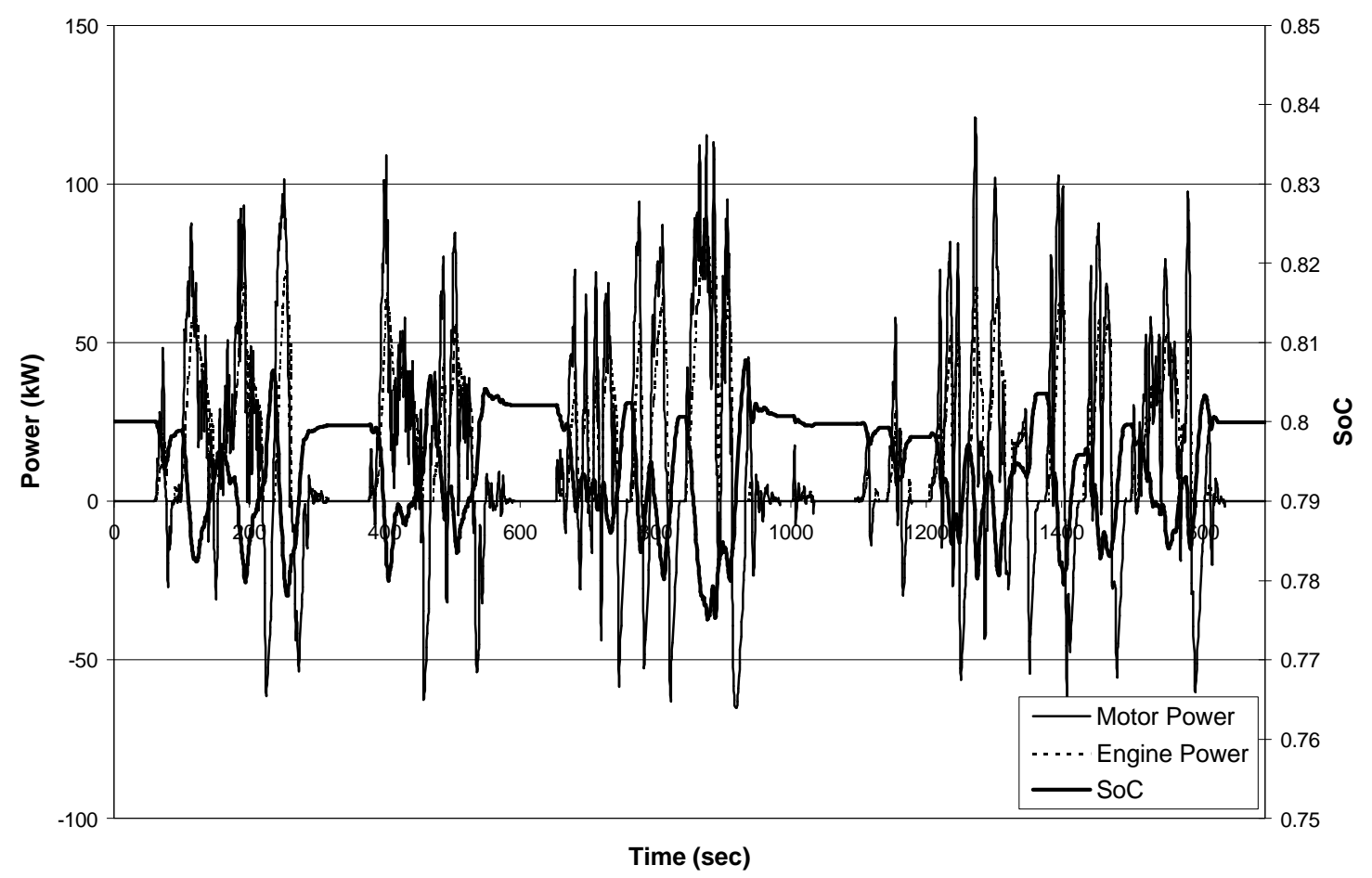

Figure A.17 Class 6 Series HEV on CSHVC without auxiliary load.

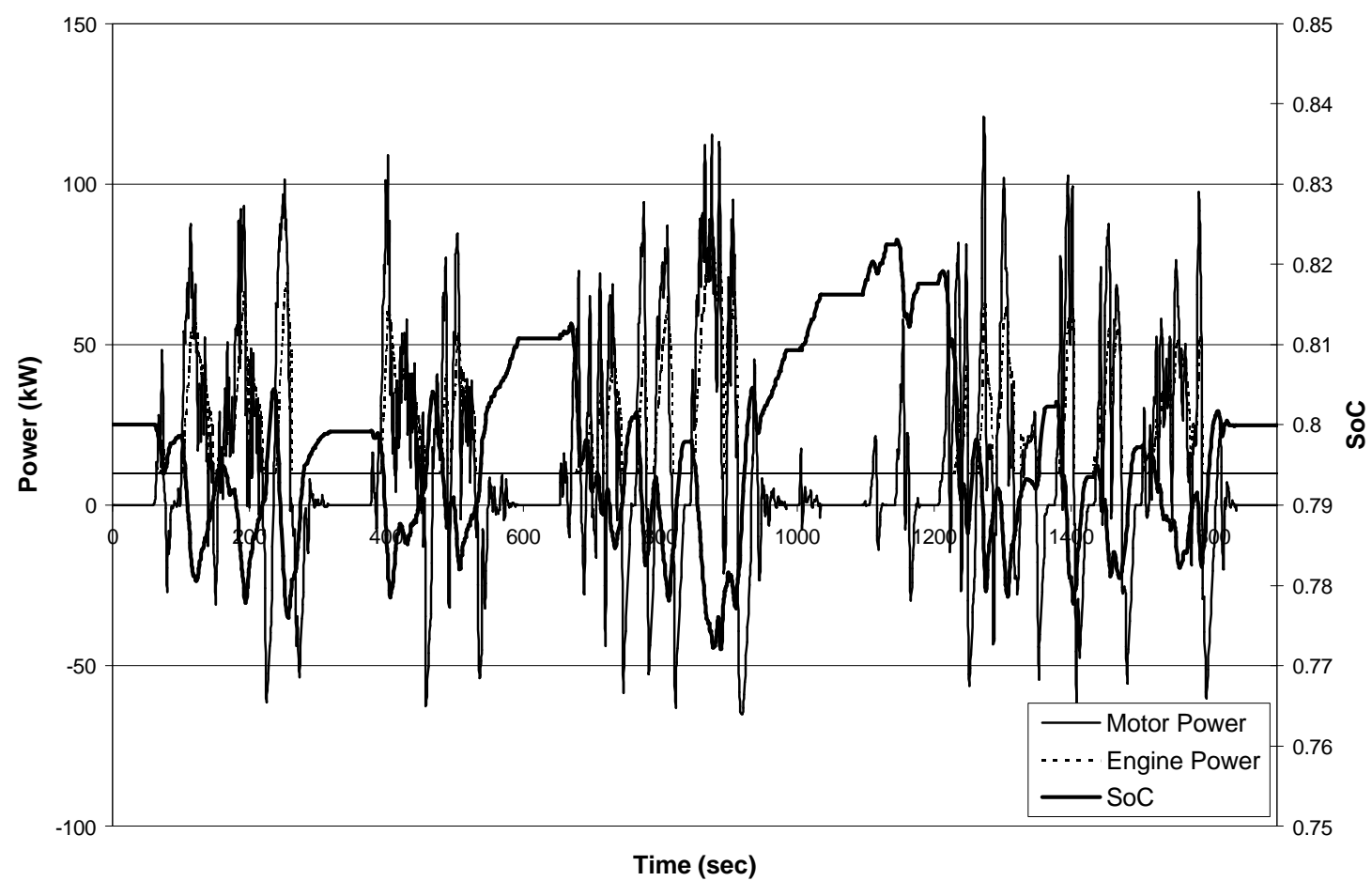

Figure A.18 Class 6 Series HEV on CSHVC with auxiliary load. 


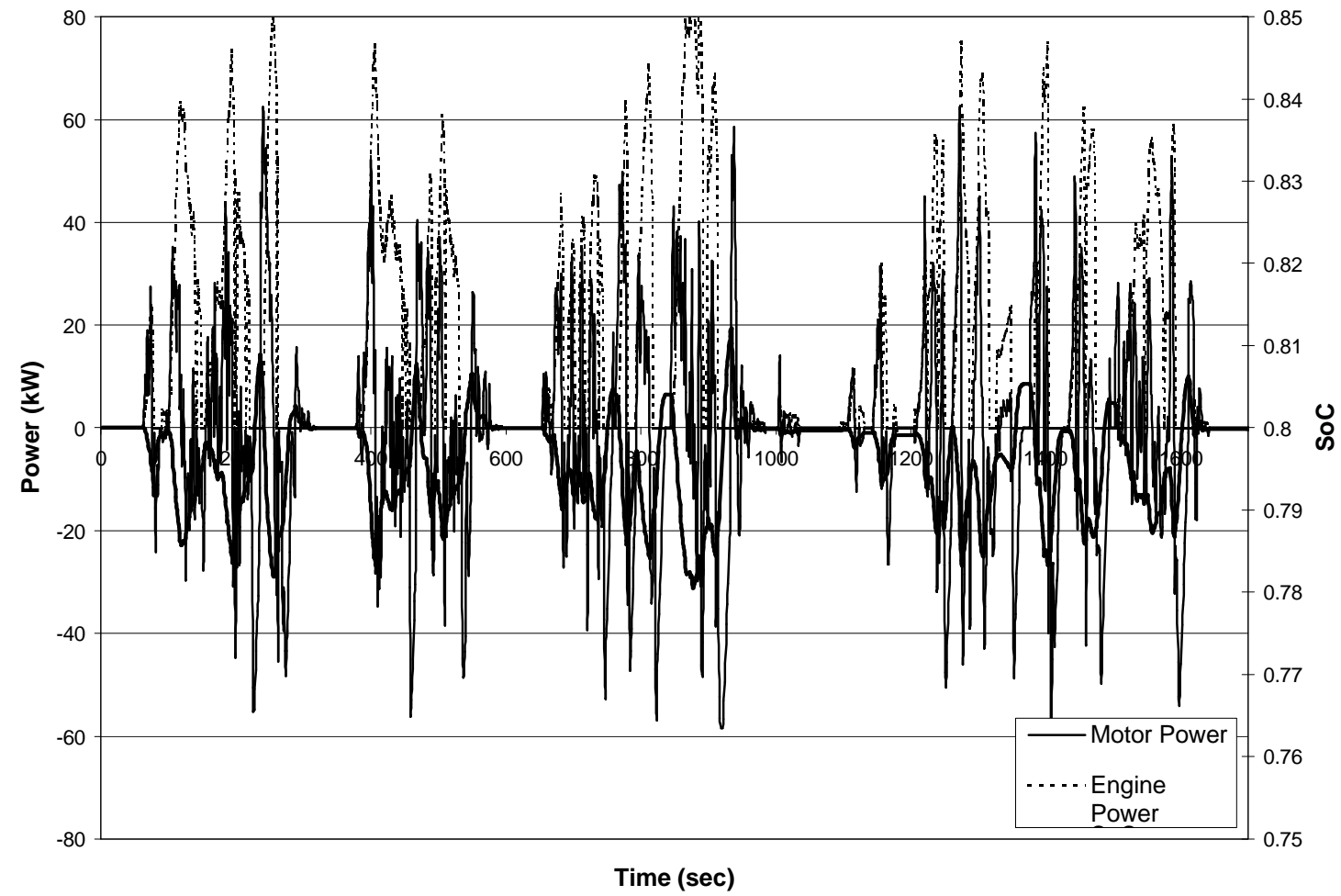

Figure A.19 Class 6 Parallel HEV on CSHVC without auxiliary load.

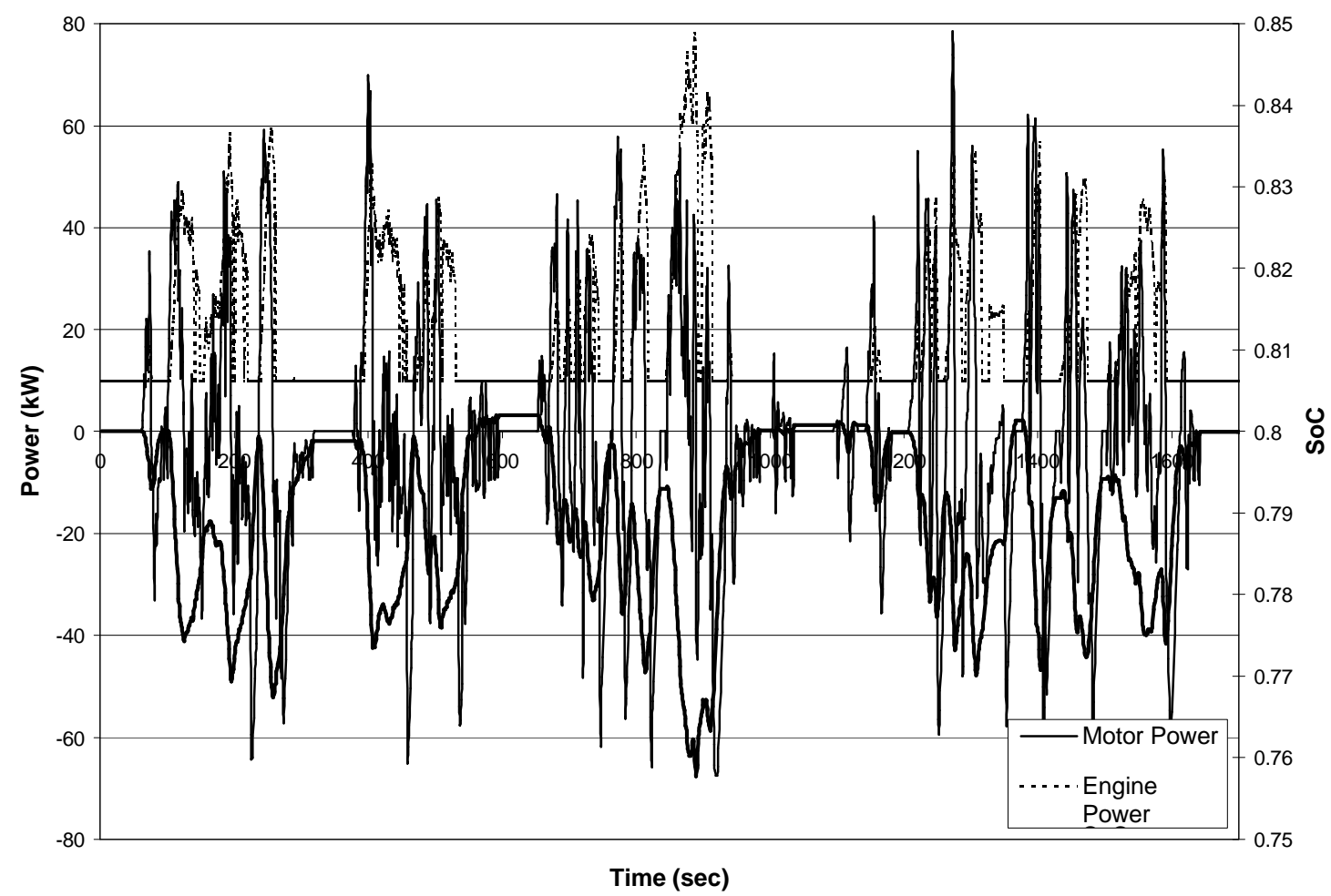

Figure A.20 Class 6 Parallel HEV on CSHVC with auxiliary load. 


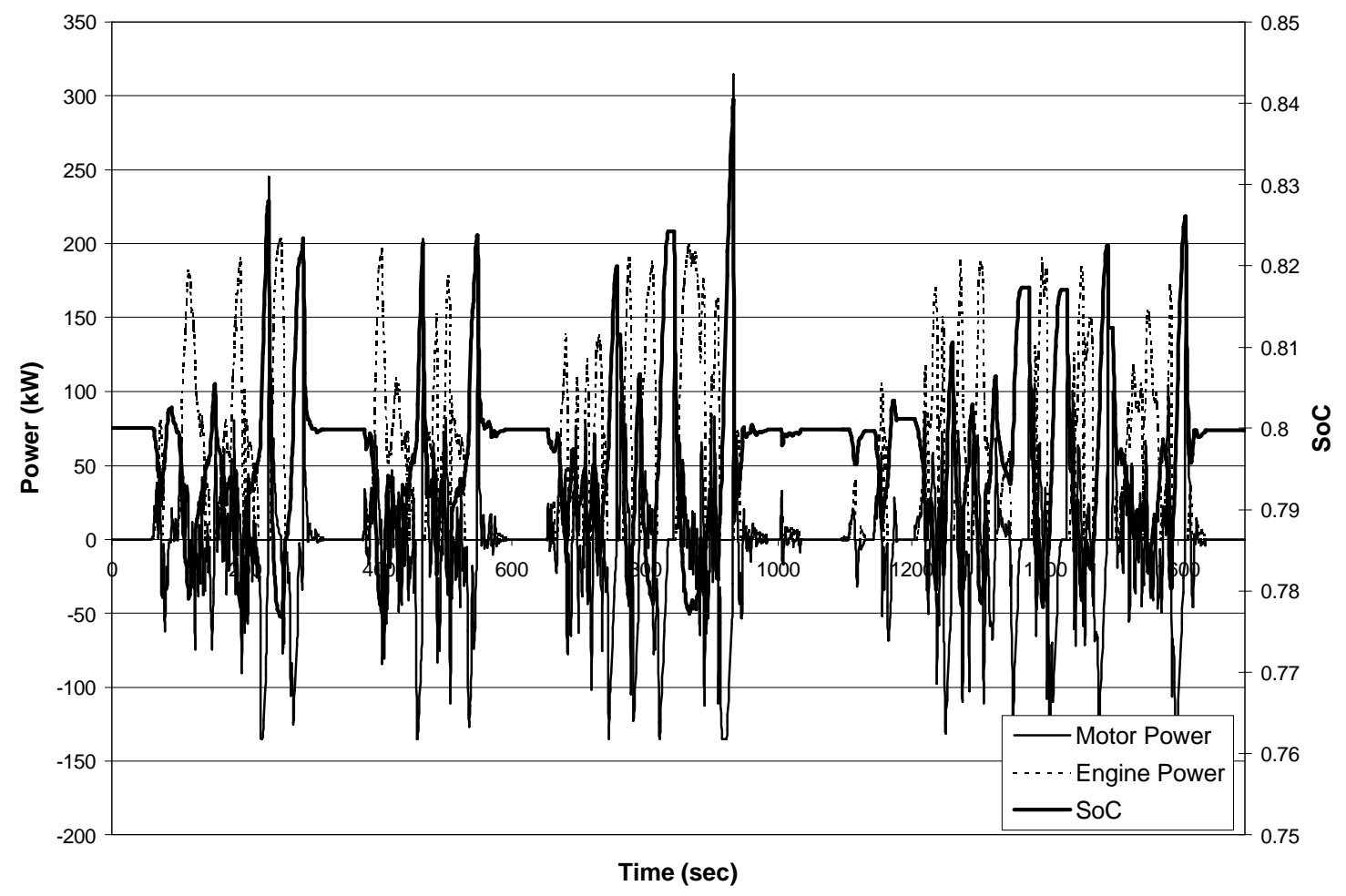

Figure A.21 Class 8 Series HEV on CSHVC without auxiliary load.

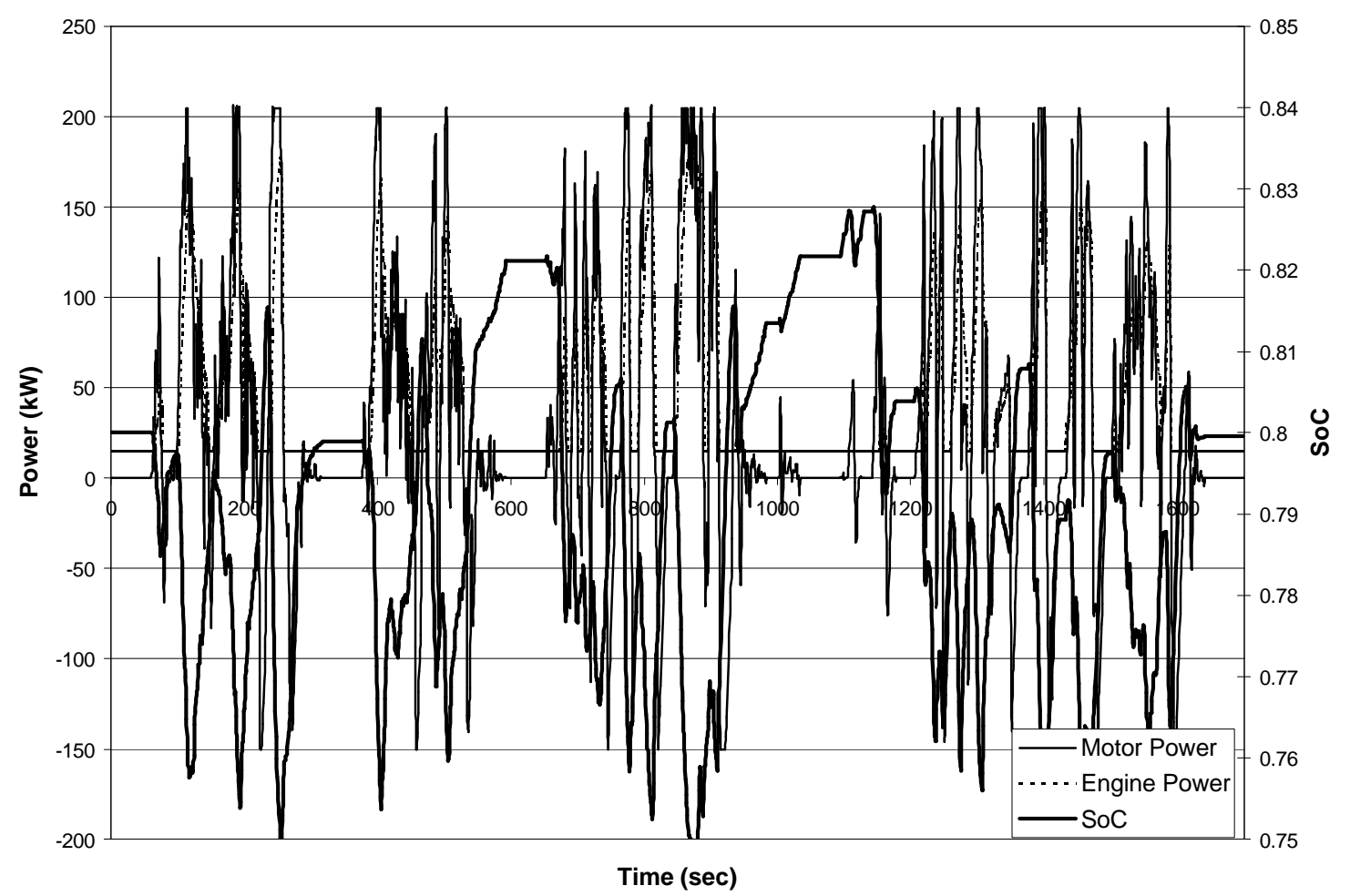

Figure A.22 Class 8 Series HEV on CSHVC with auxiliary load. 


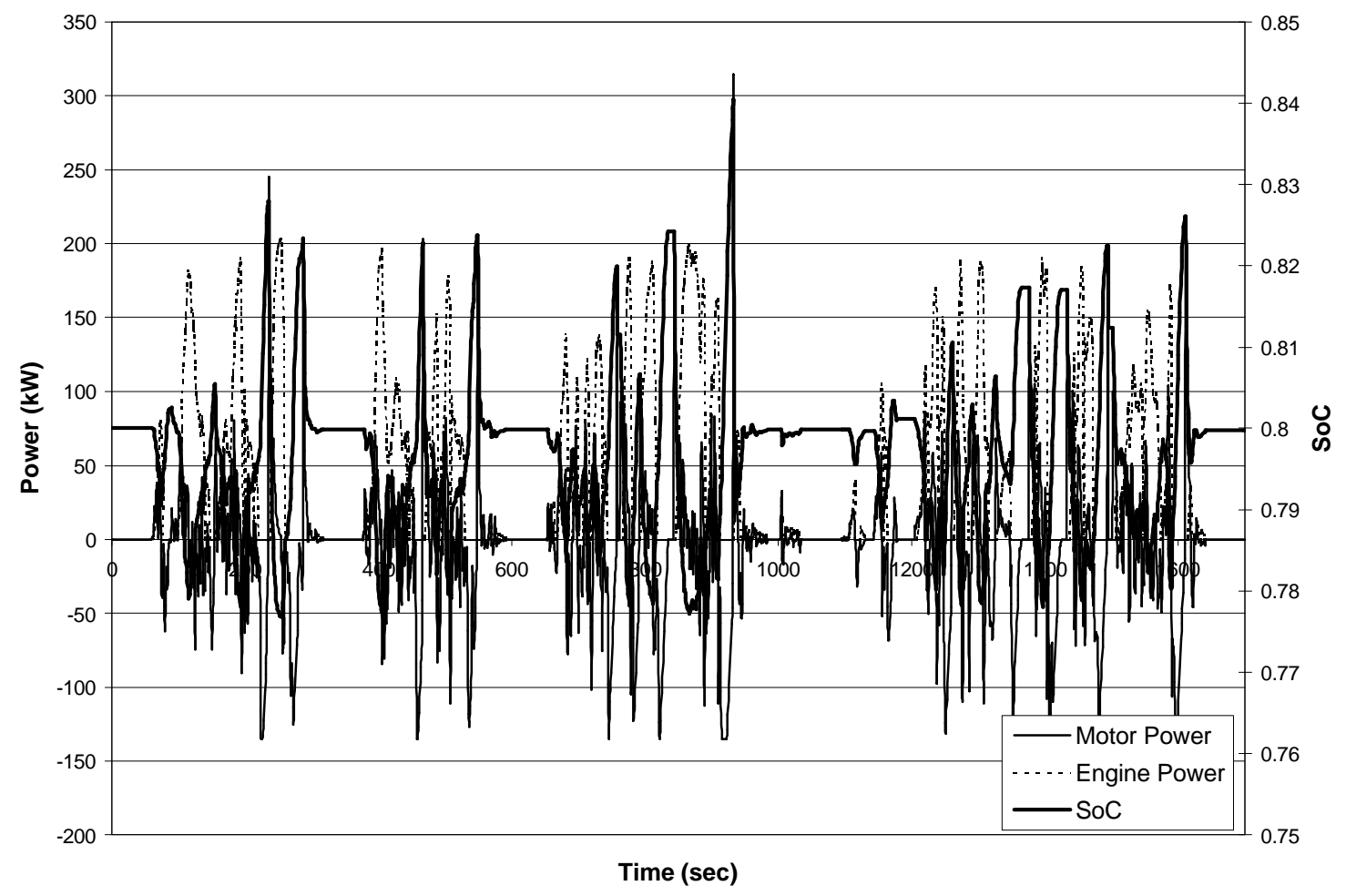

Figure A.23 Class 8 Parallel HEV on CSHVC without auxiliary load.

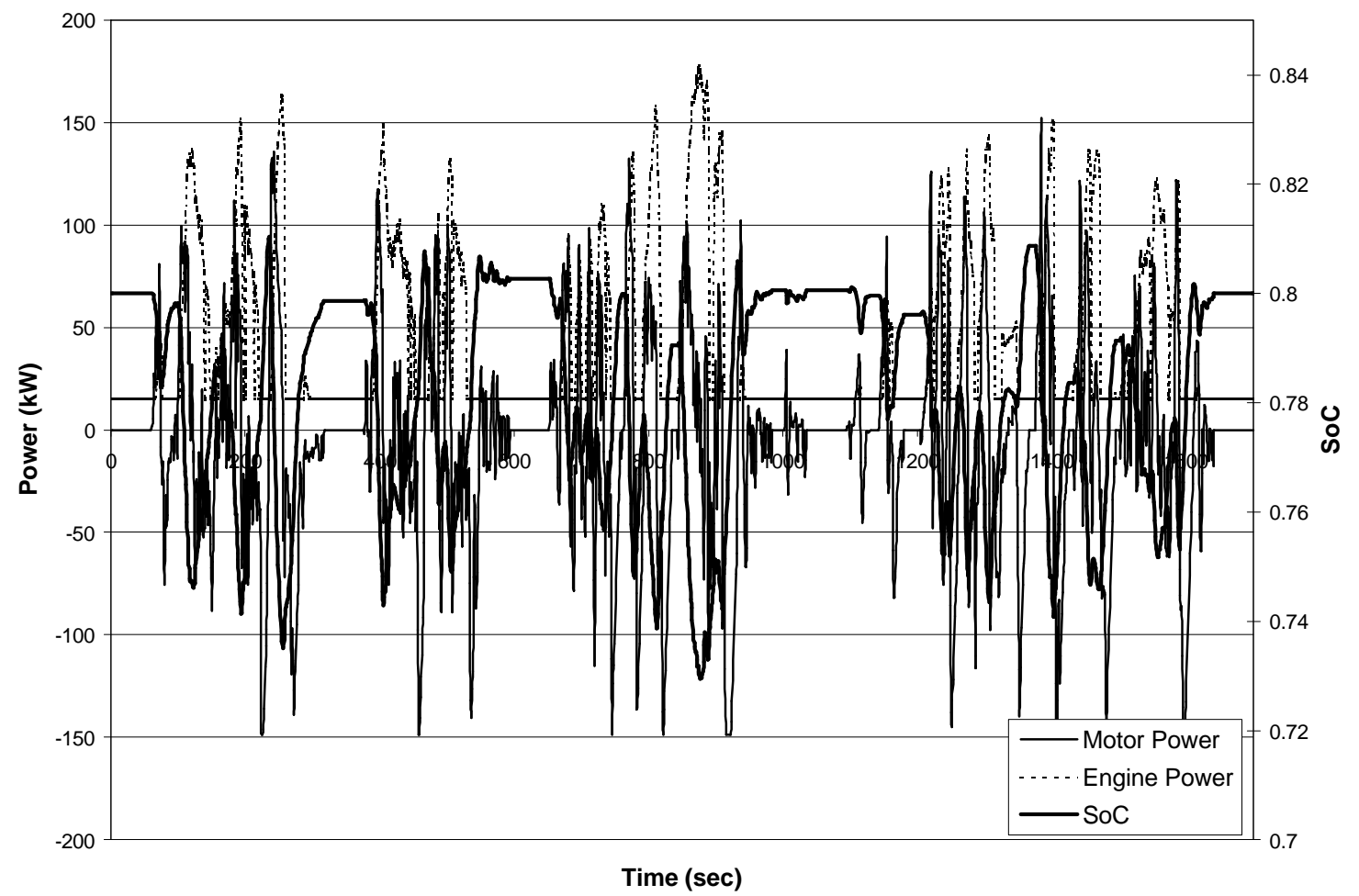

Figure A.24 Class 8 Parallel HEV on CSHVC with auxiliary load. 


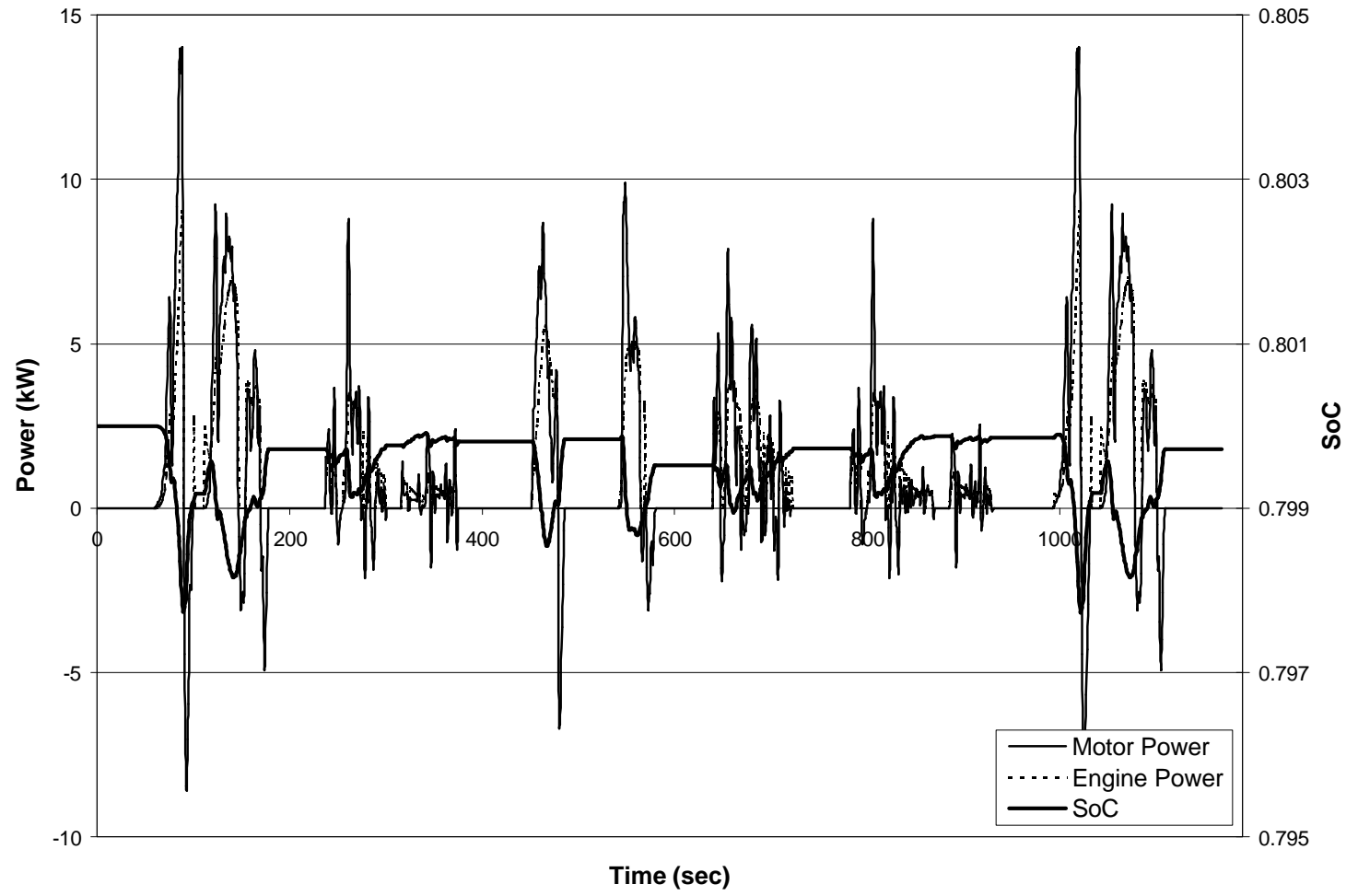

Figure A.25 Class 2B Series HEV on Yard Cycle without auxiliary load.

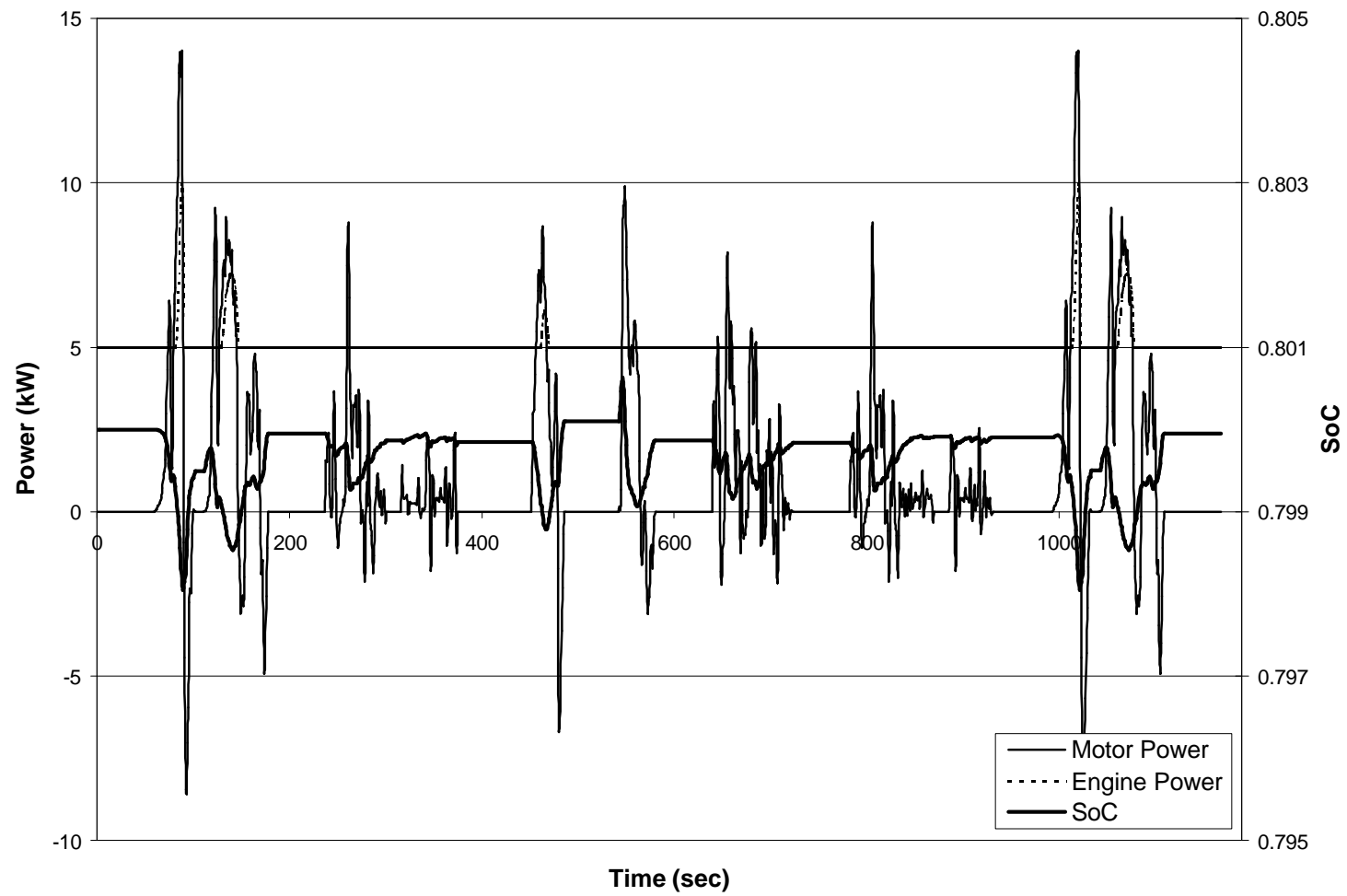

Figure A.26 Class 2B Series HEV on Yard Cycle with auxiliary load. 


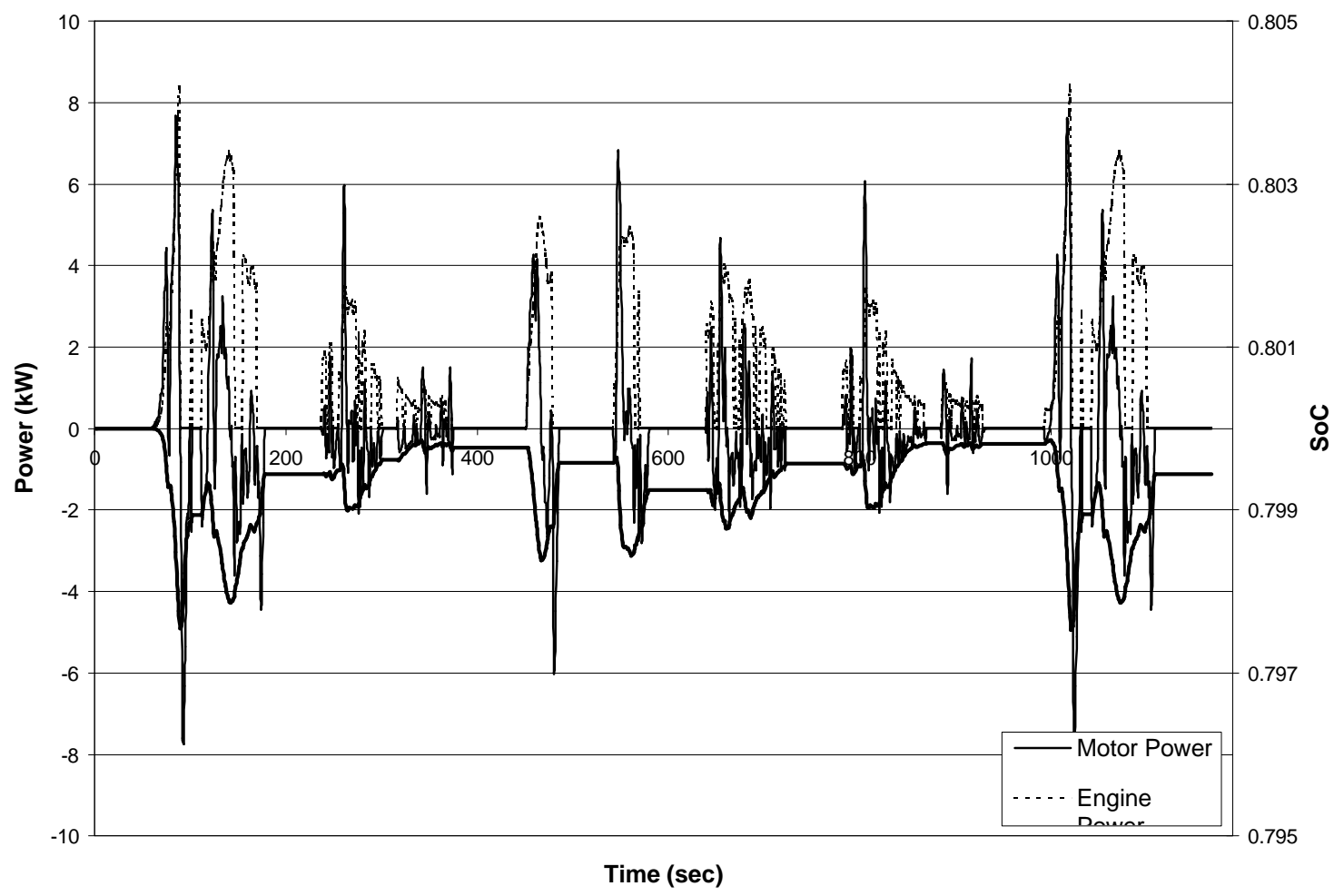

Figure A.27 Class 2B Parallel HEV on Yard Cycle without auxiliary load.

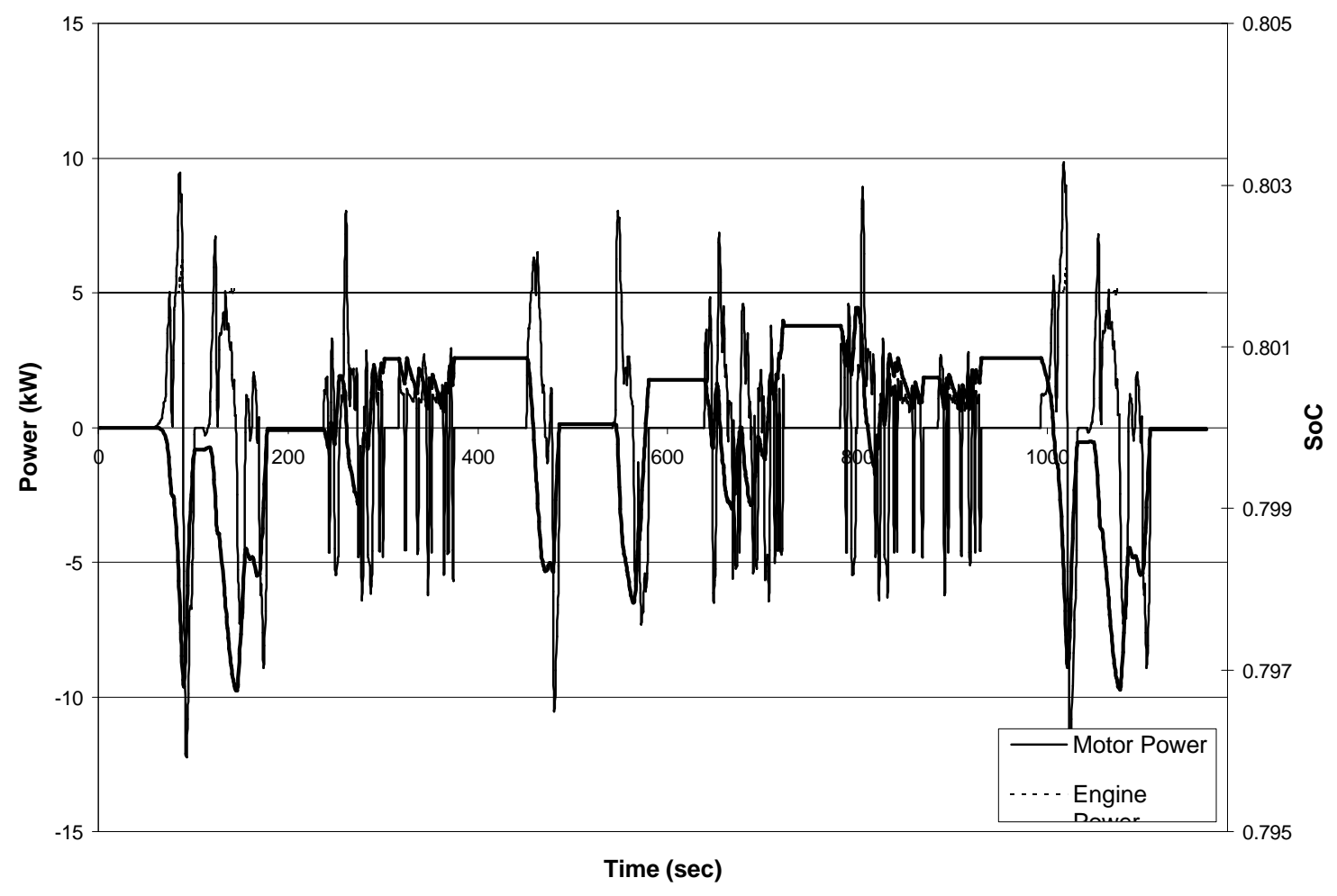

Figure A.28 Class 2B Parallel HEV on Yard Cycle with auxiliary load. 


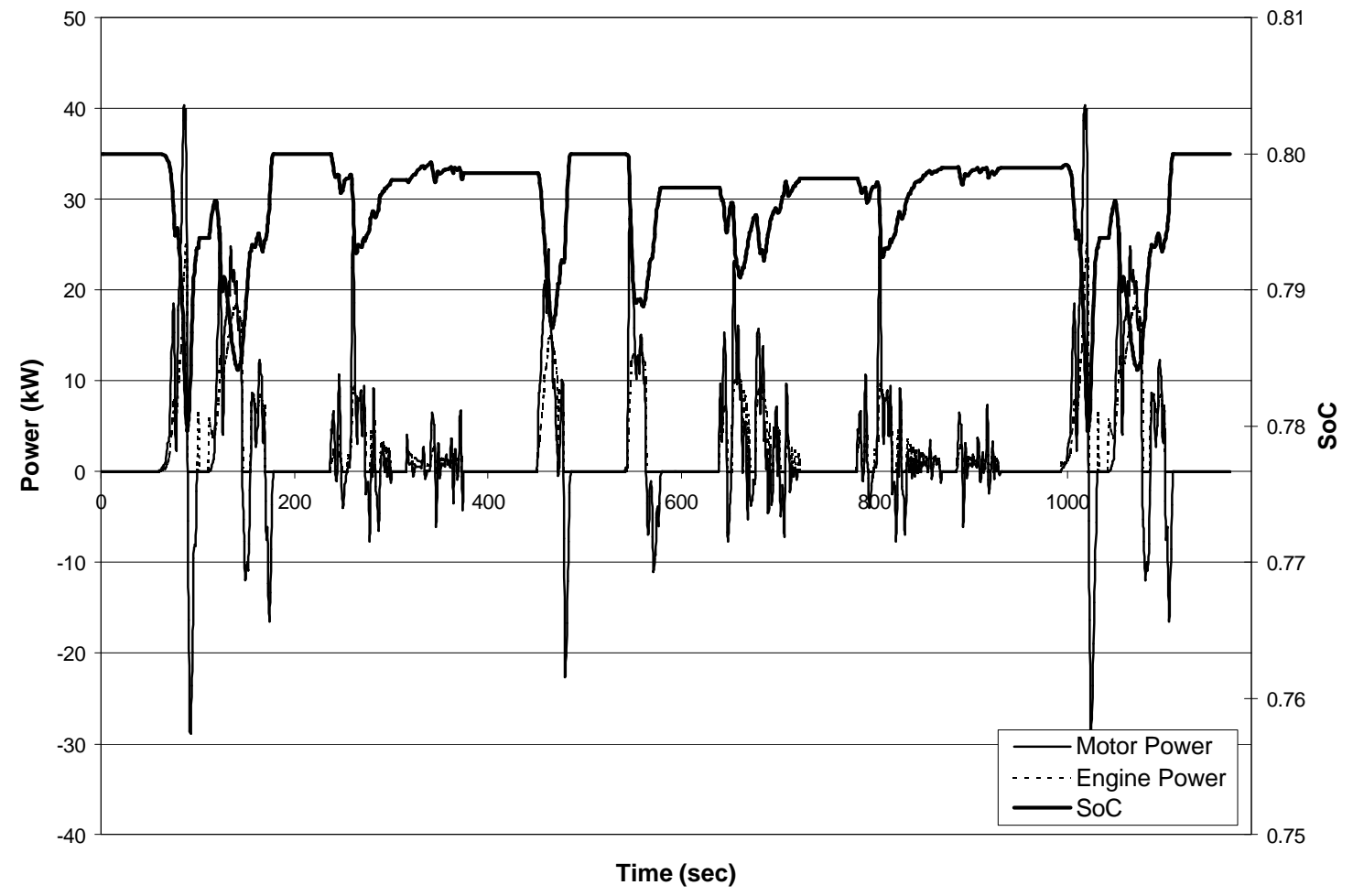

Figure A.29 Class 6 Series HEV on Yard Cycle without auxiliary load.

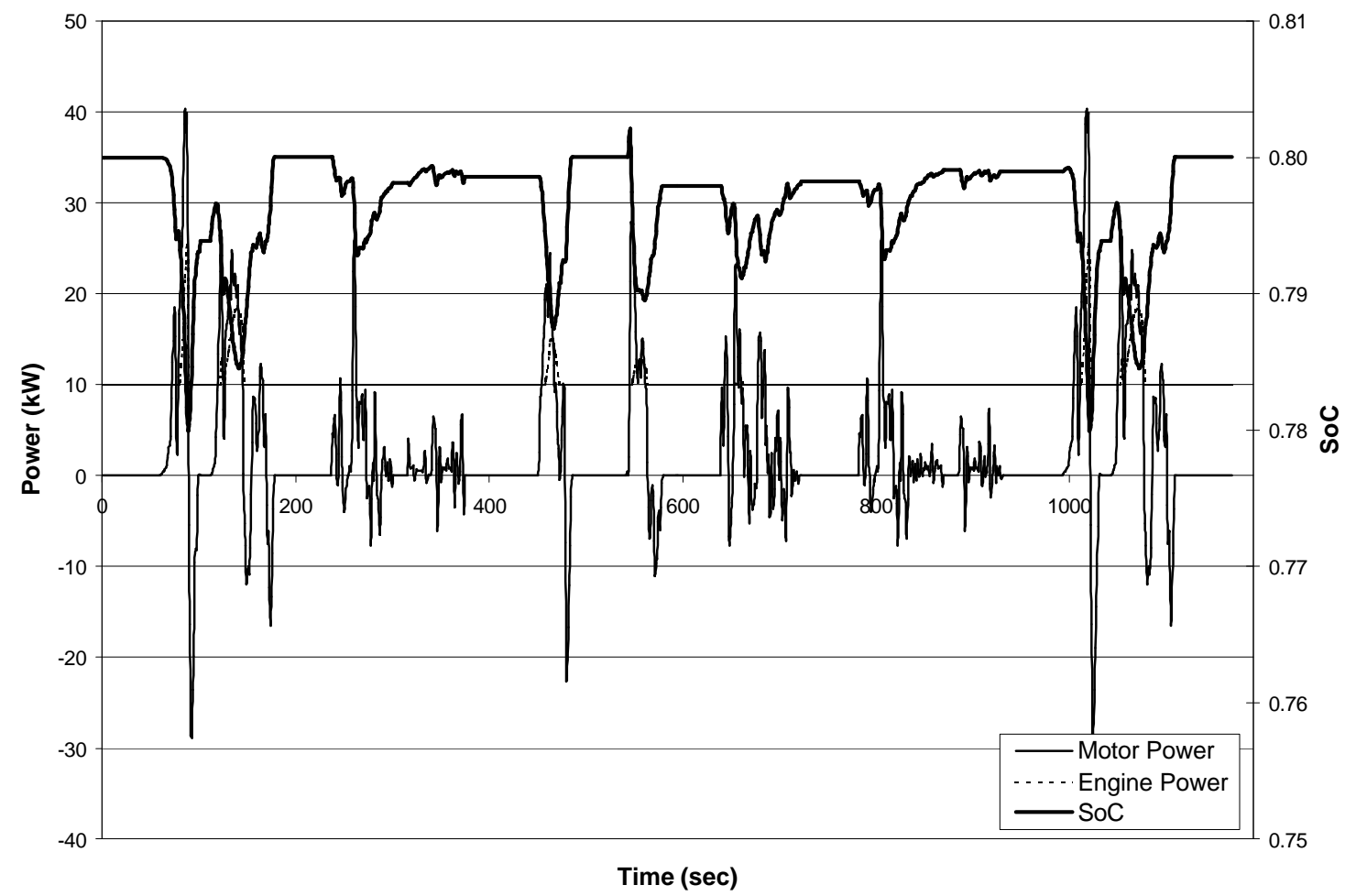

Figure A.30 Class 6 Series HEV on Yard Cycle with auxiliary load. 


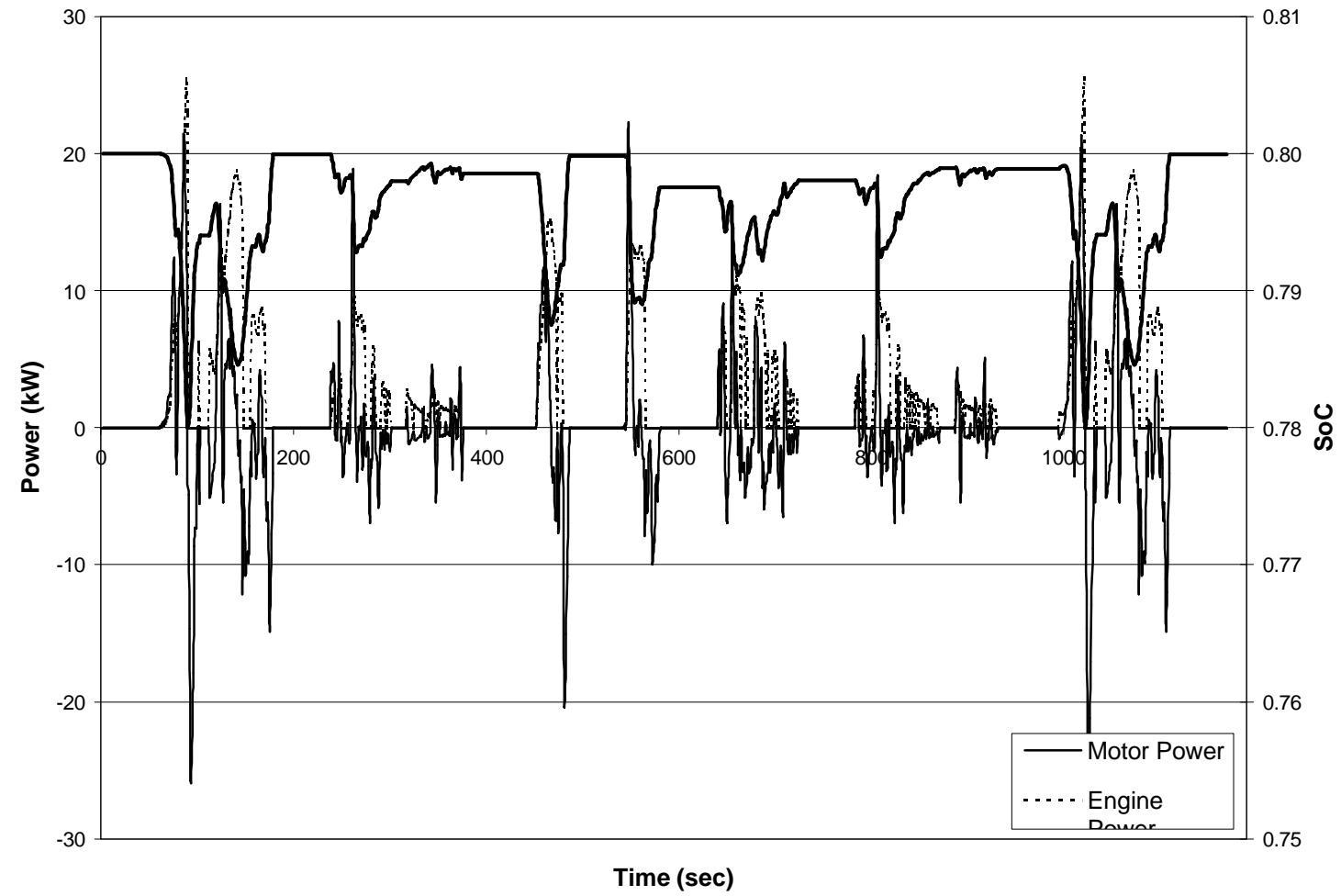

Figure A.31 Class 6 Parallel HEV on Yard Cycle without auxiliary load.

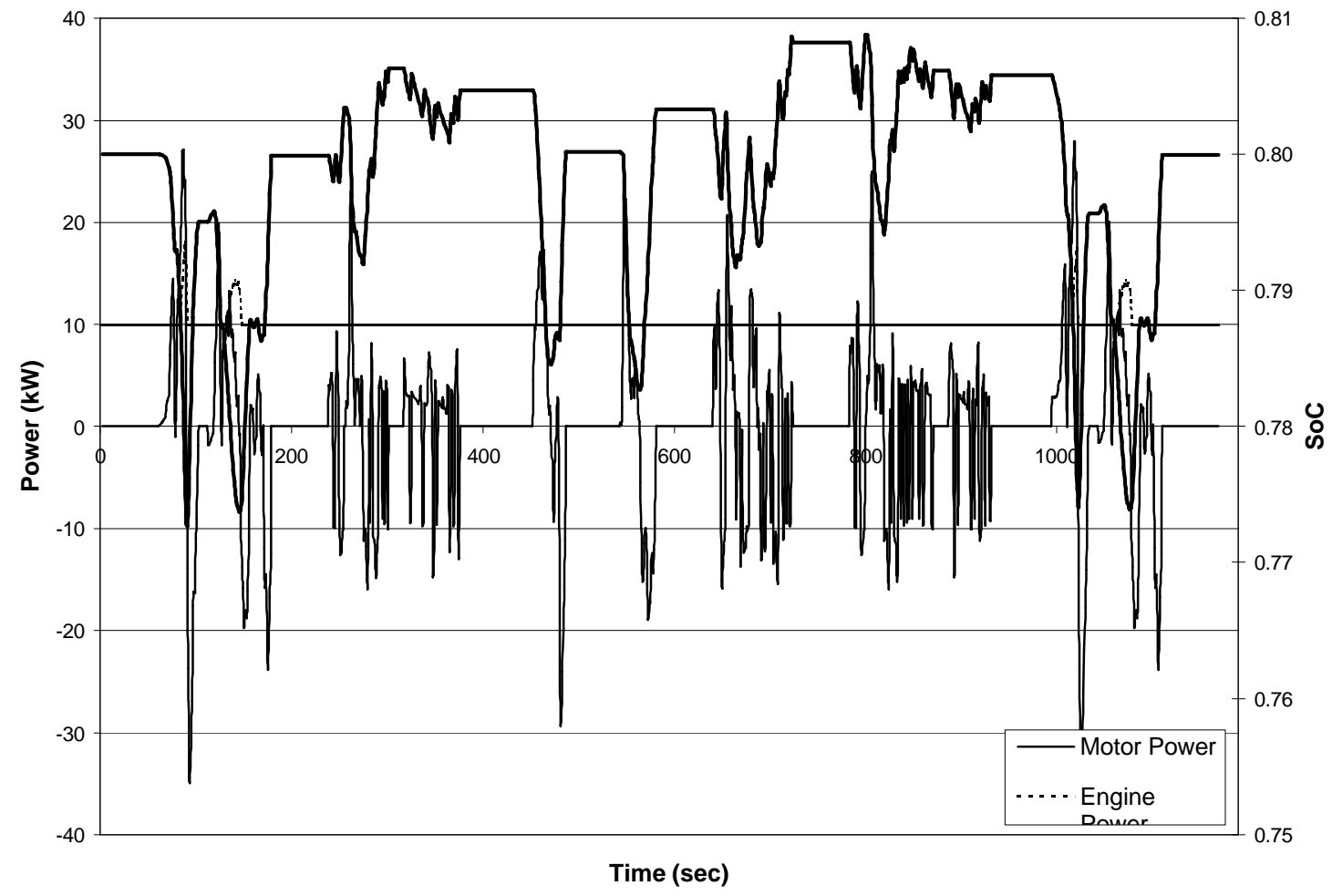

Figure A.32 Class 6 Parallel HEV on Yard Cycle with auxiliary load. 


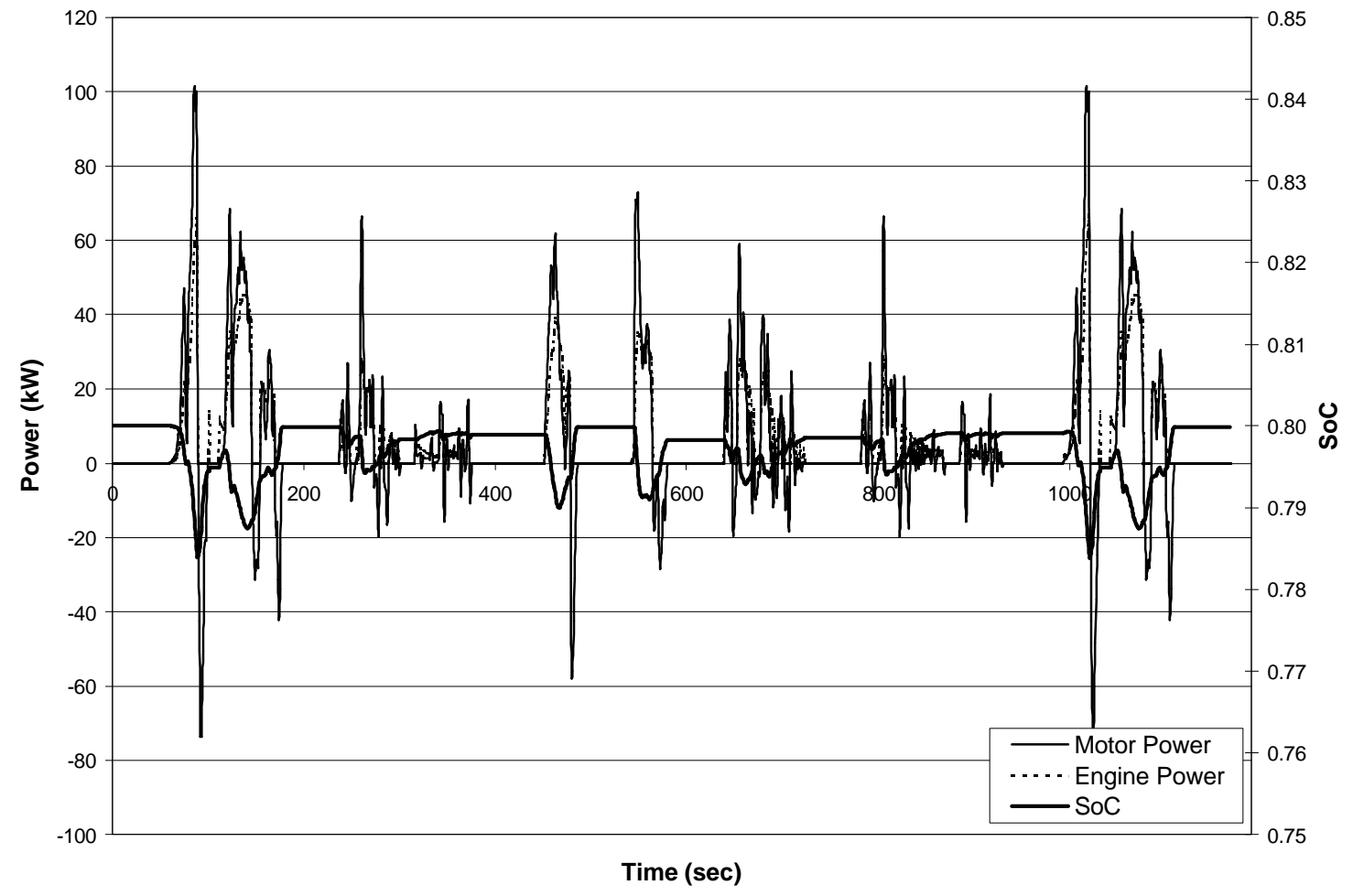

Figure A.33 Class 8 Series HEV on Yard Cycle without auxiliary load.

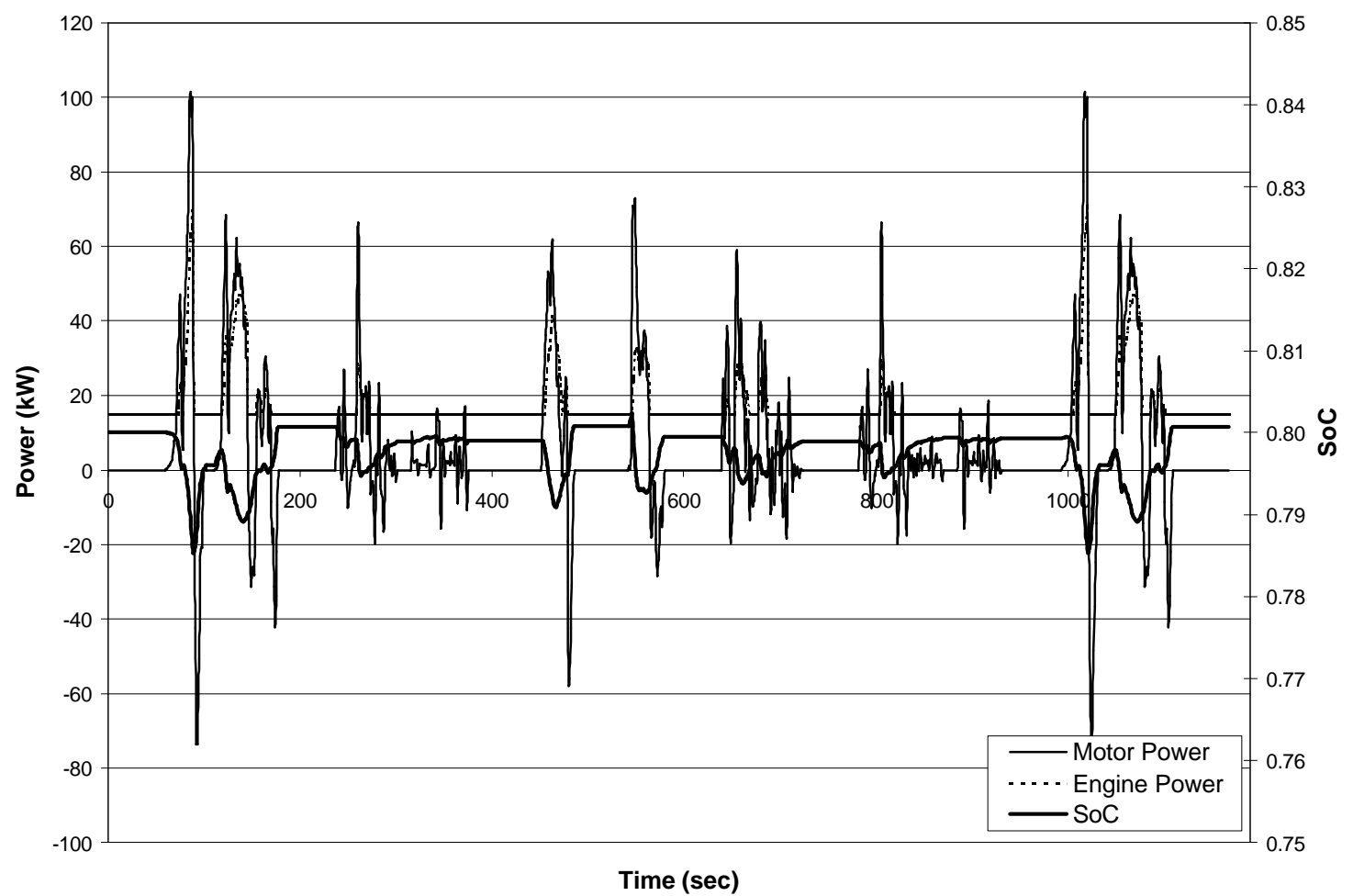

Figure A.34 Class 8 Series HEV on Yard Cycle with auxiliary load. 


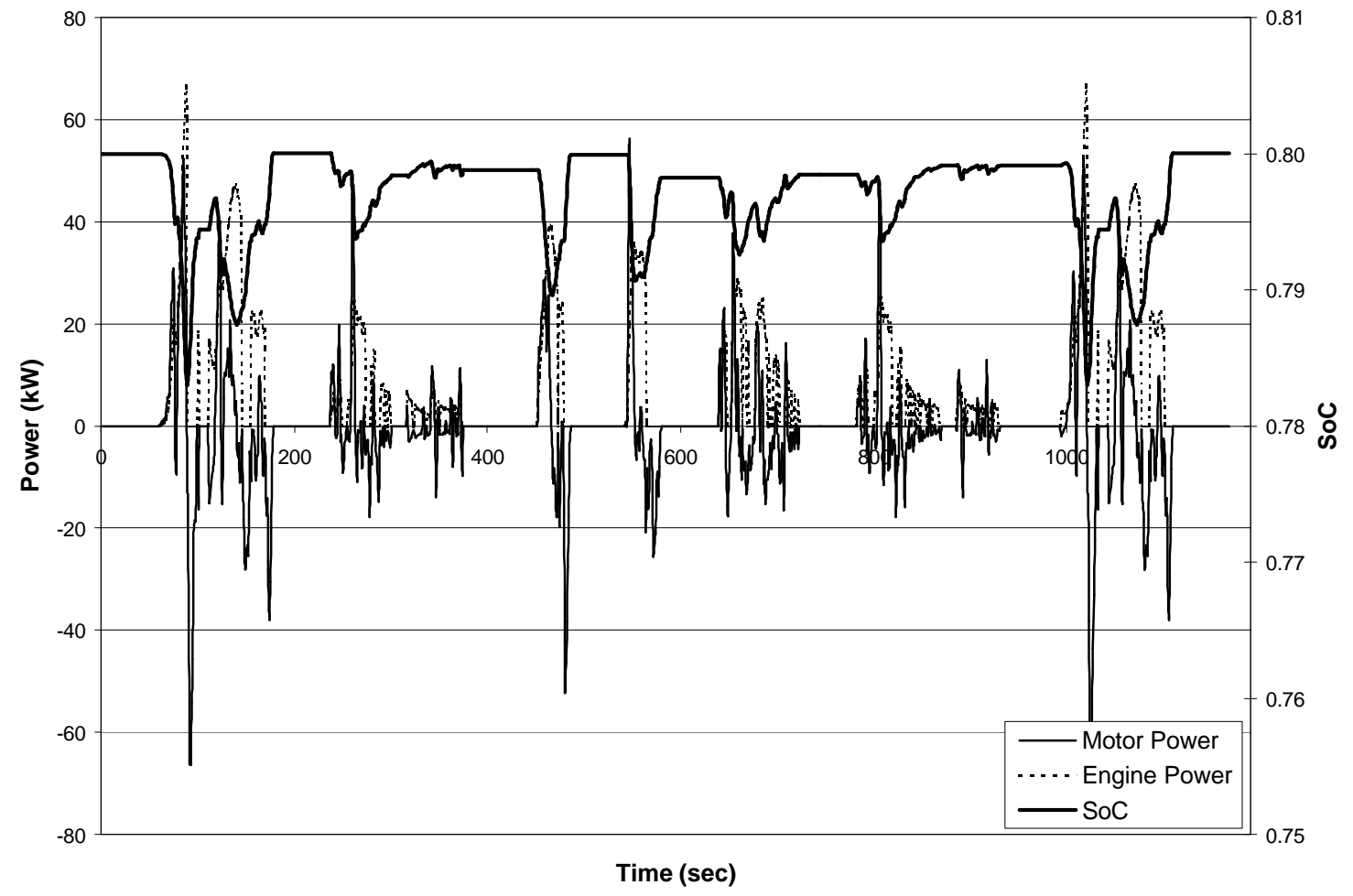

Figure A.35 Class 8 Parallel HEV on Yard Cycle without auxiliary load.

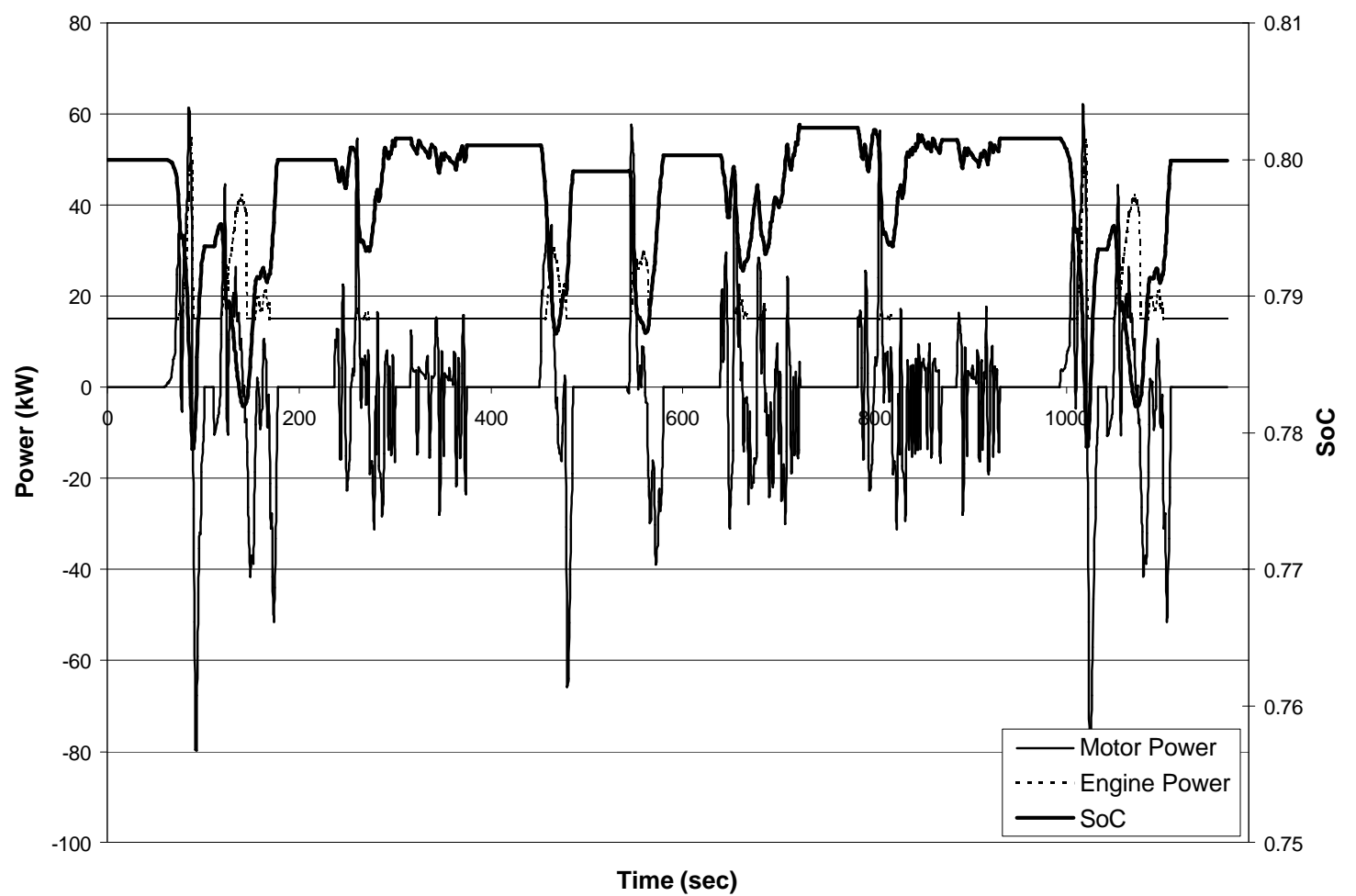

Figure A.36 Class 8 Parallel HEV on Yard Cycle with auxiliary load. 


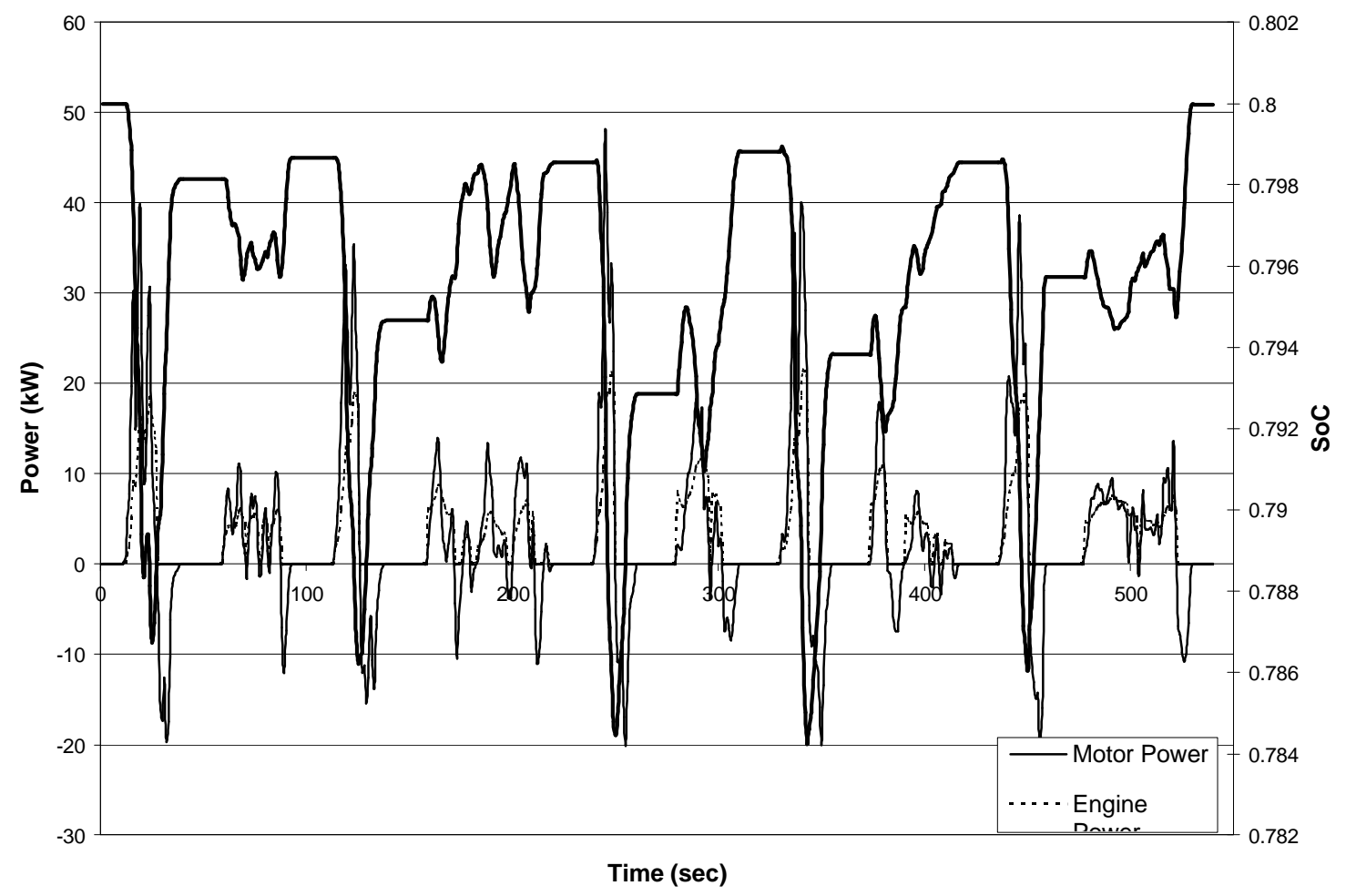

Figure A.37 Class 2B Series HEV on Manhattan Cycle without auxiliary load.

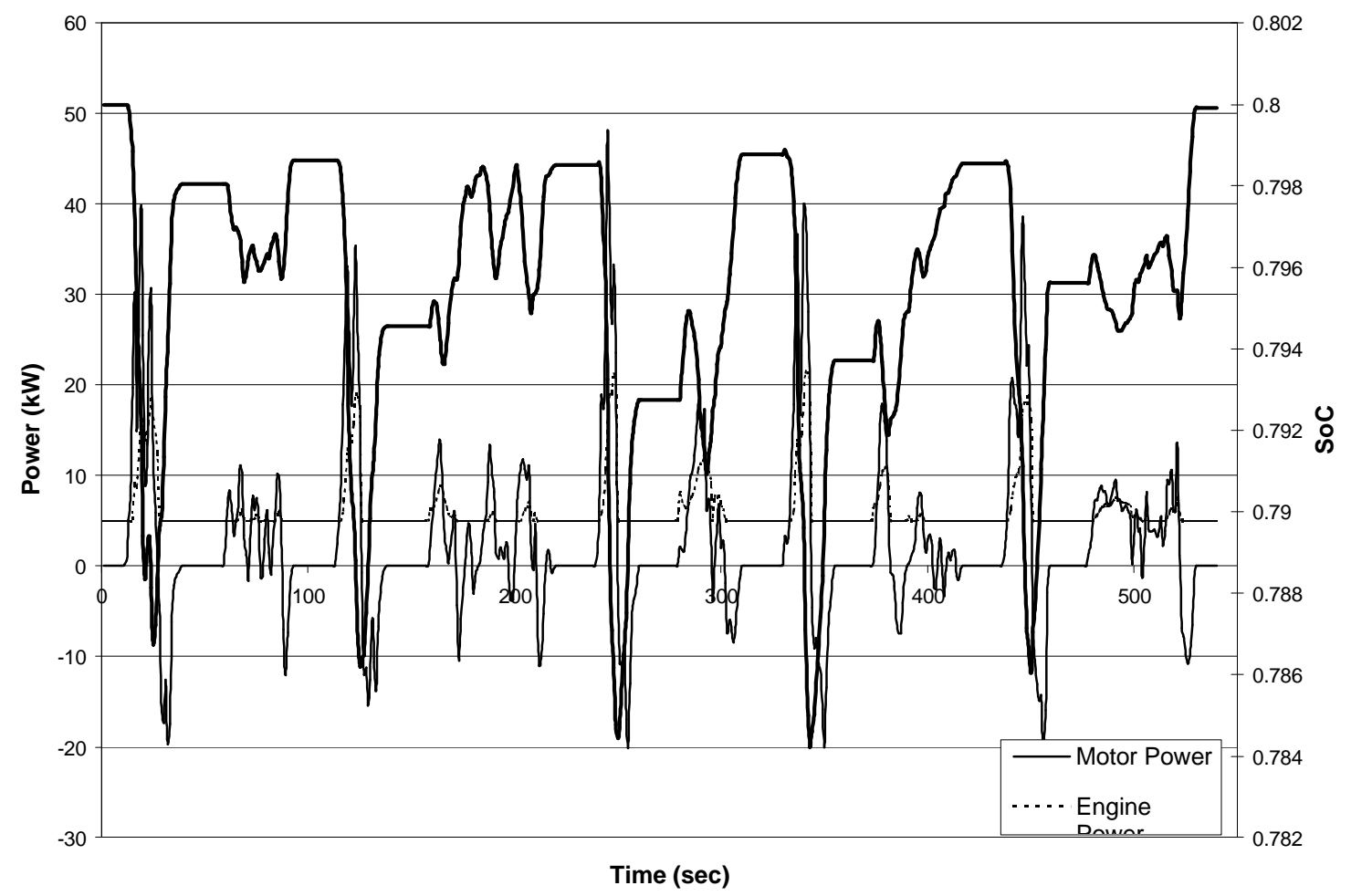

Figure A.38 Class 2B Series HEV on Manhattan Cycle with auxiliary load. 


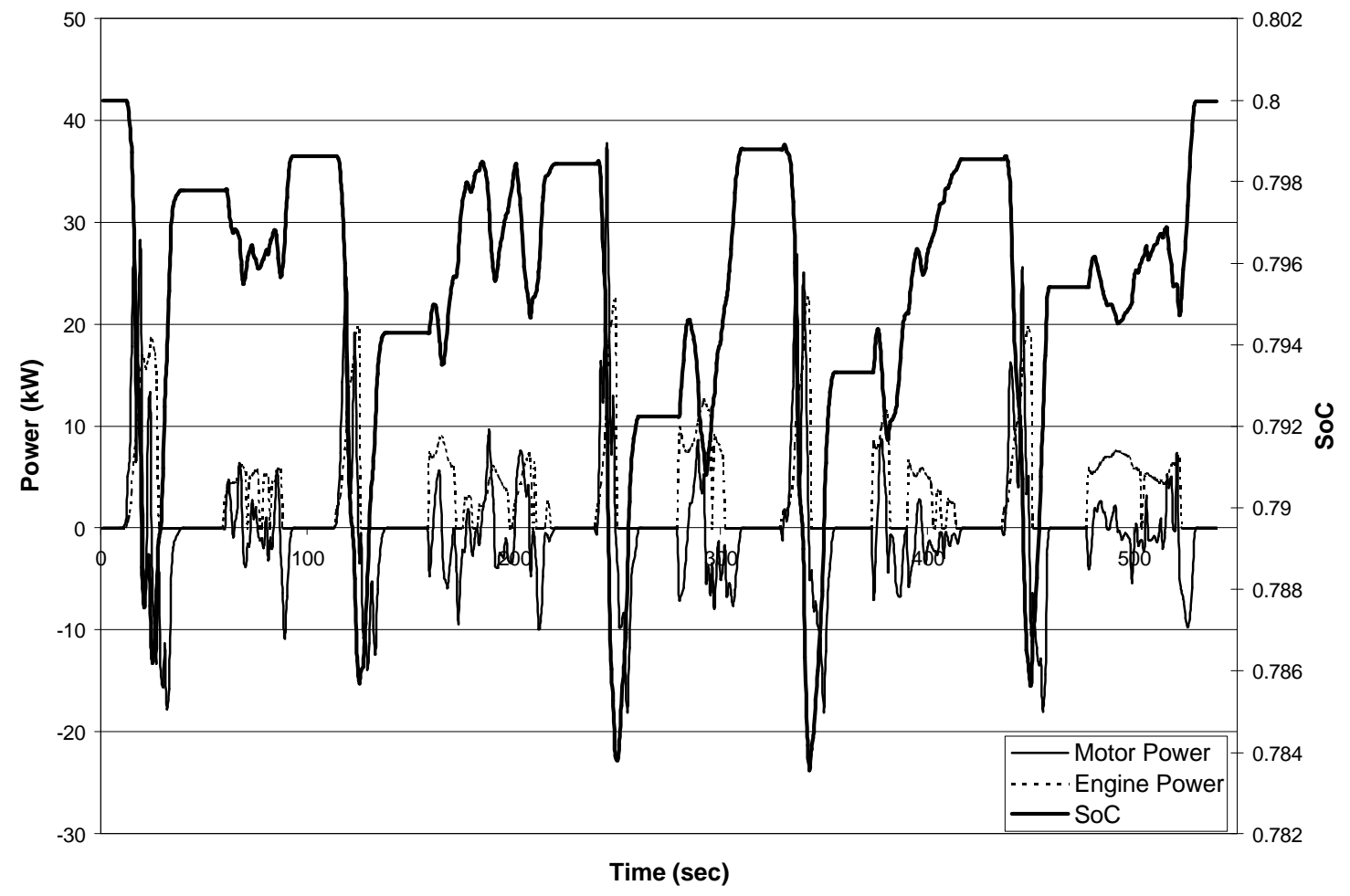

Figure A.39 Class 2B Parallel HEV on Manhattan Cycle without auxiliary load.

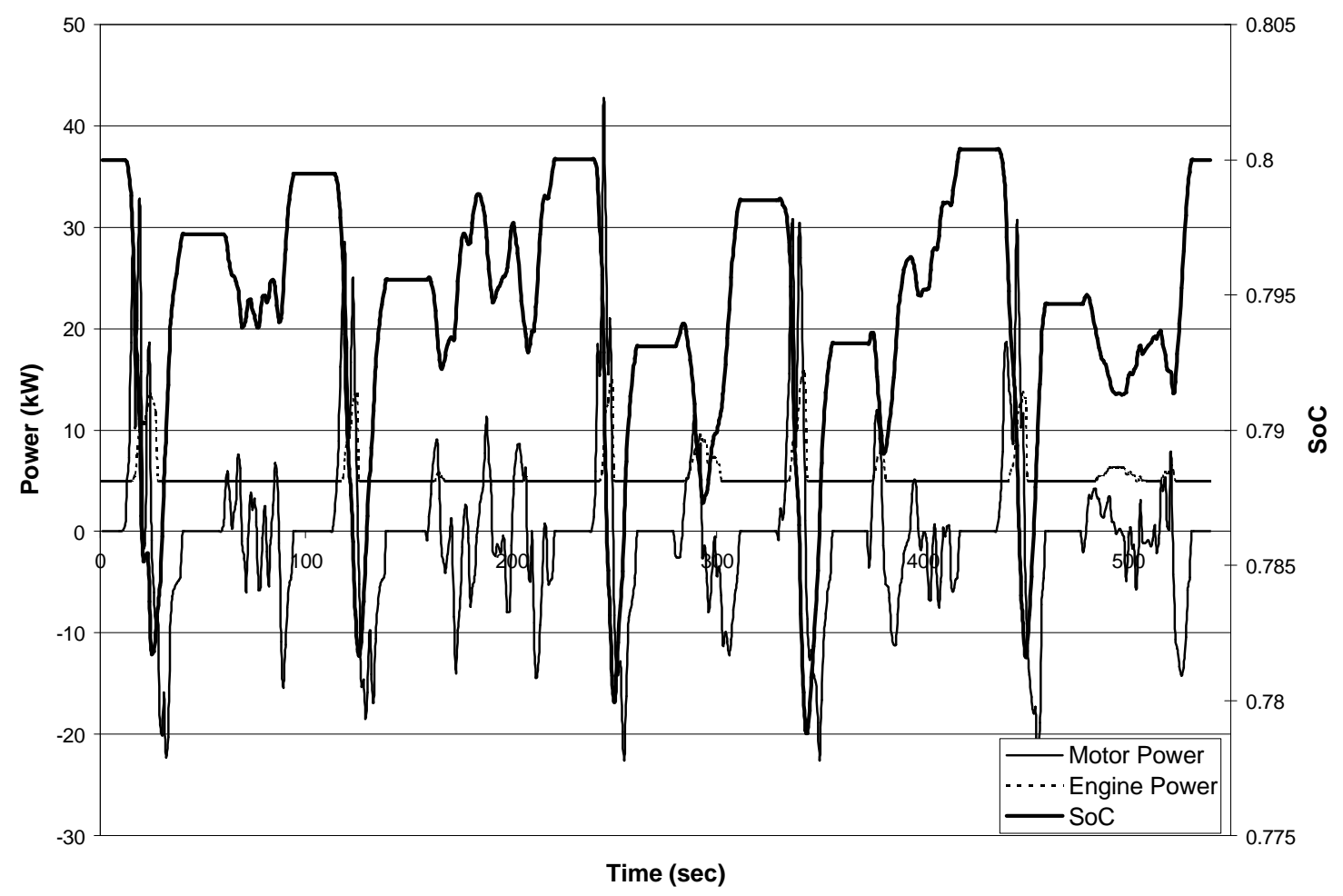

Figure A.40 Class 2B Parallel HEV on Manhattan Cycle with auxiliary load. 


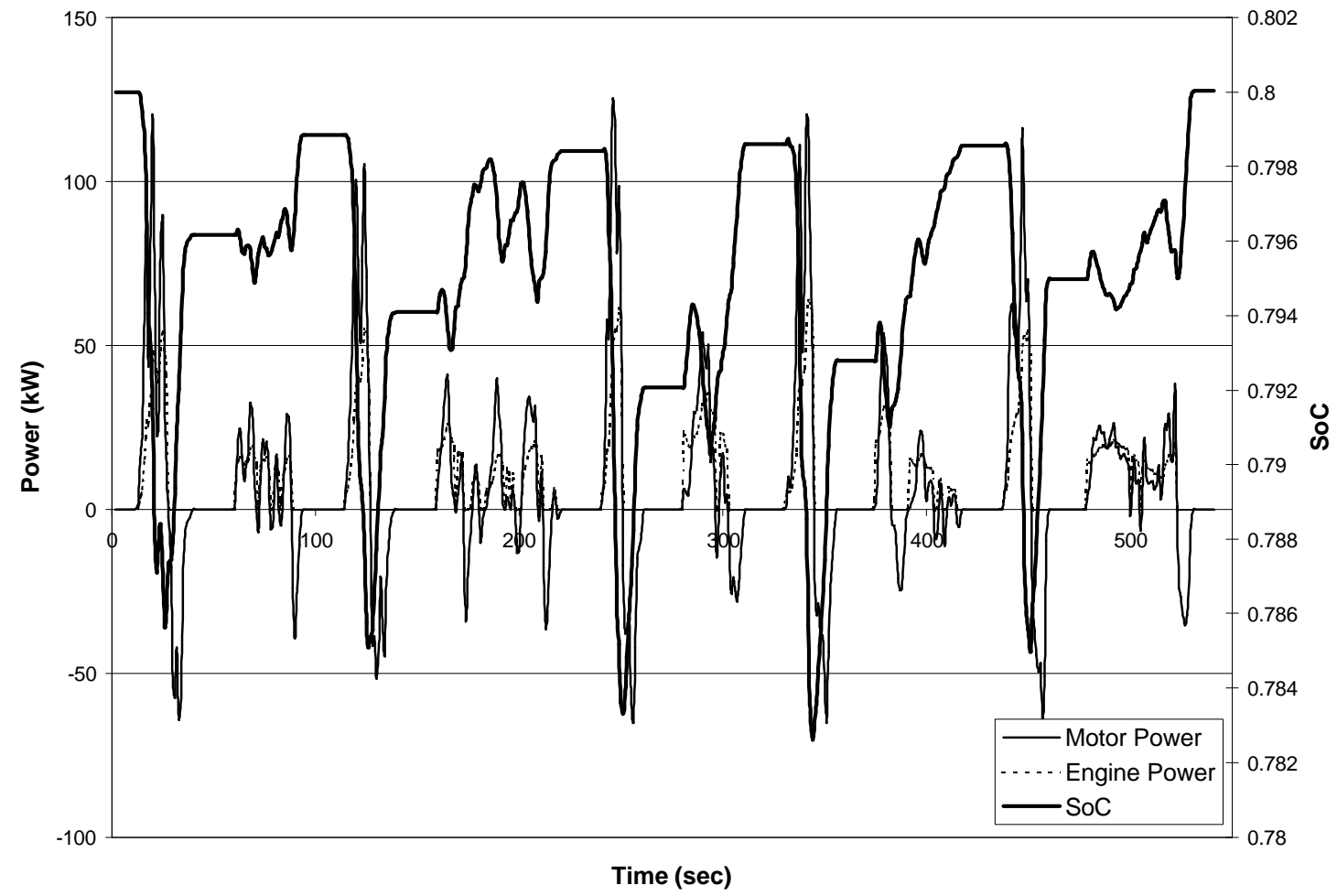

Figure A.41 Class 6 Series HEV on Manhattan Cycle without auxiliary load.

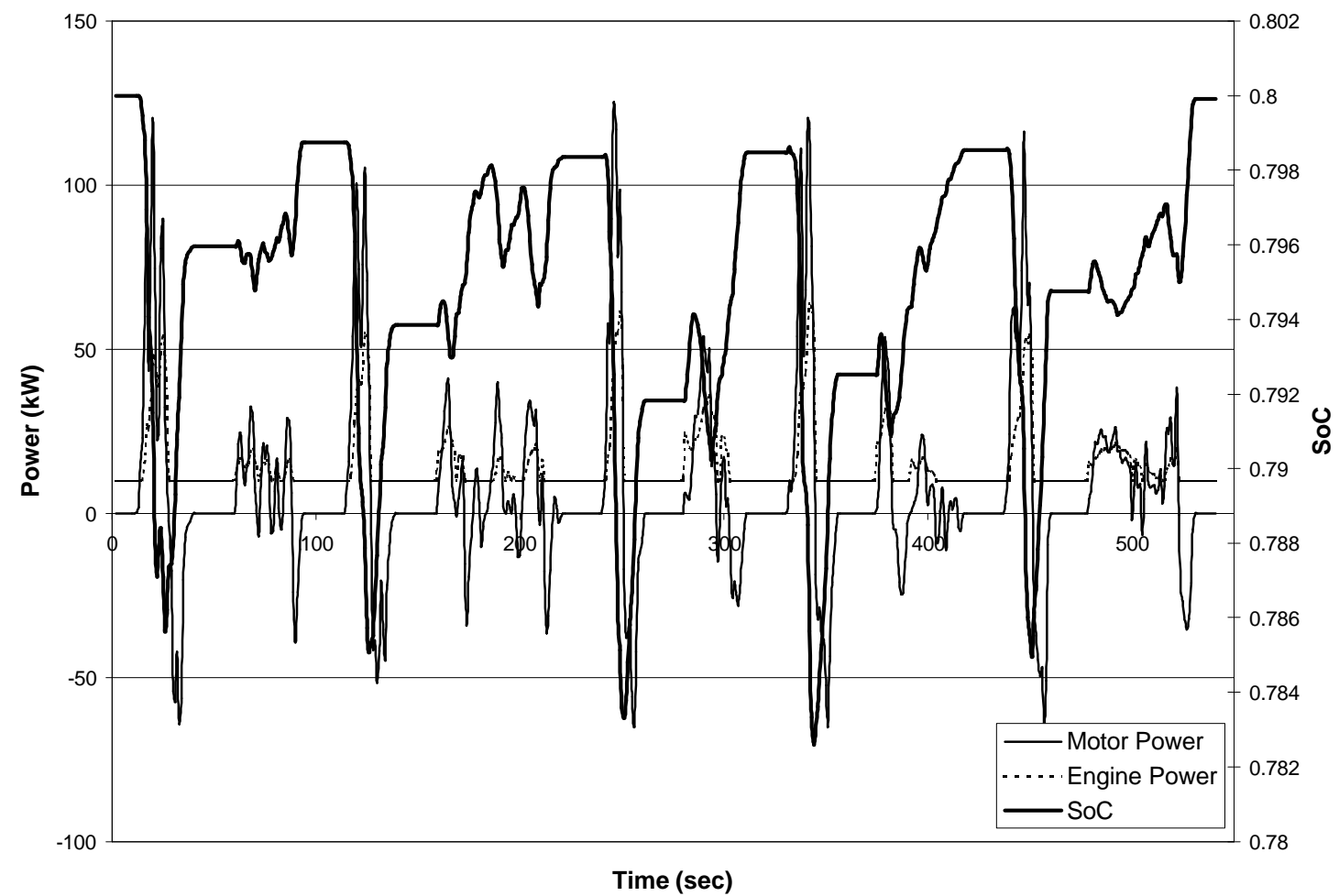

Figure A.42 Class 6 Series HEV on Manhattan Cycle with auxiliary load. 


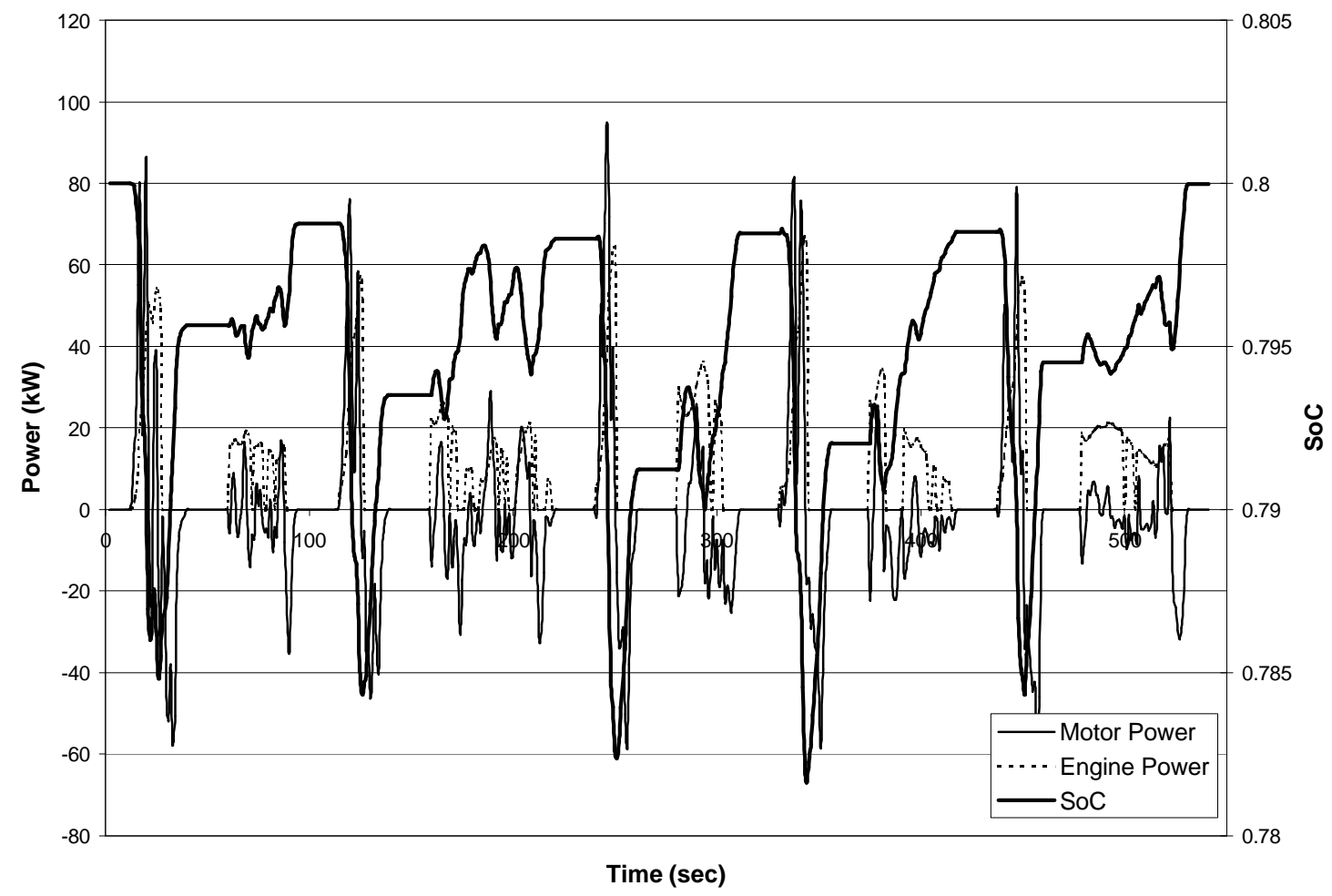

Figure A.43 Class 6 Parallel HEV on Manhattan Cycle without auxiliary load.

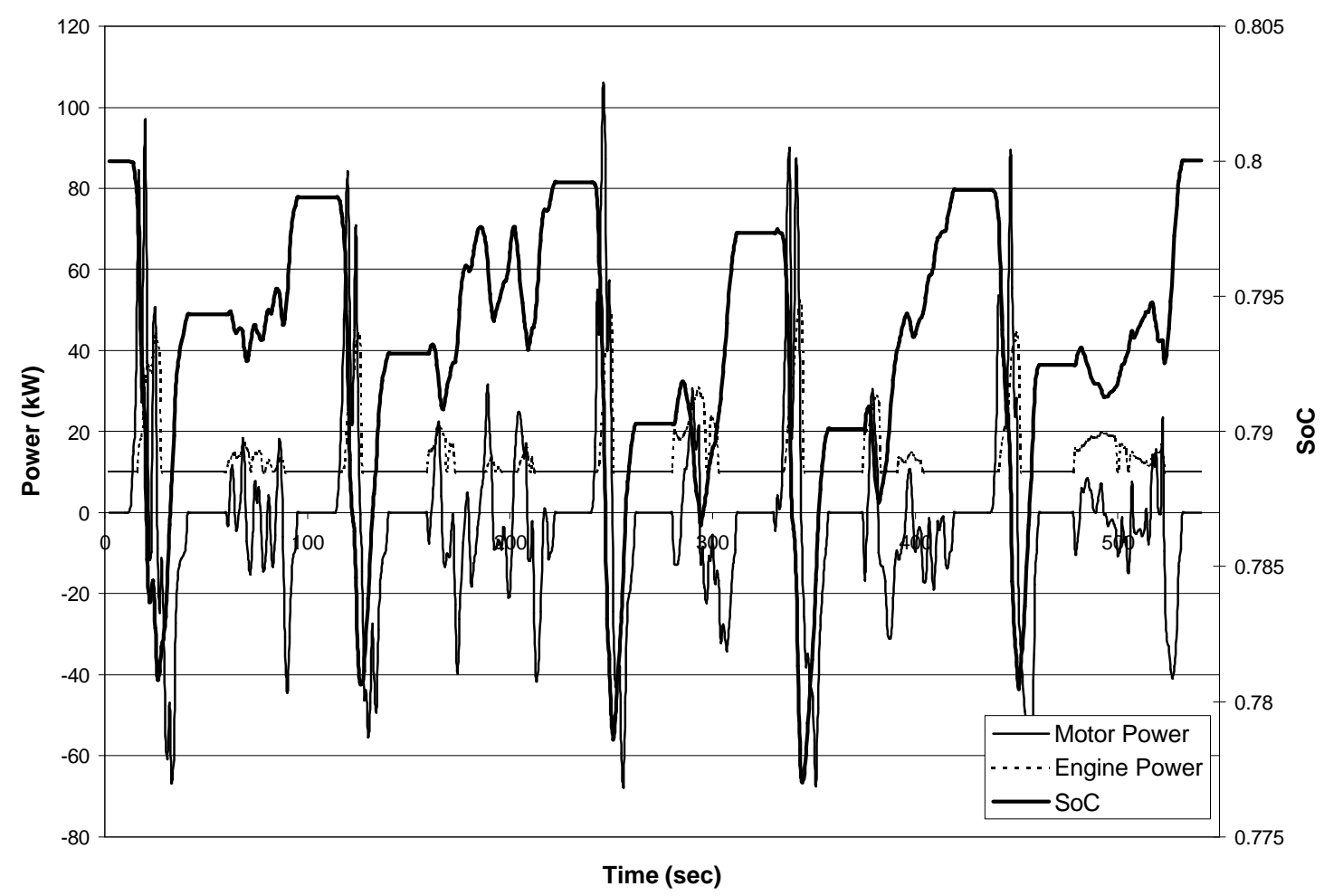

Figure A.44 Class 6 Parallel HEV on Manhattan Cycle with auxiliary load. 


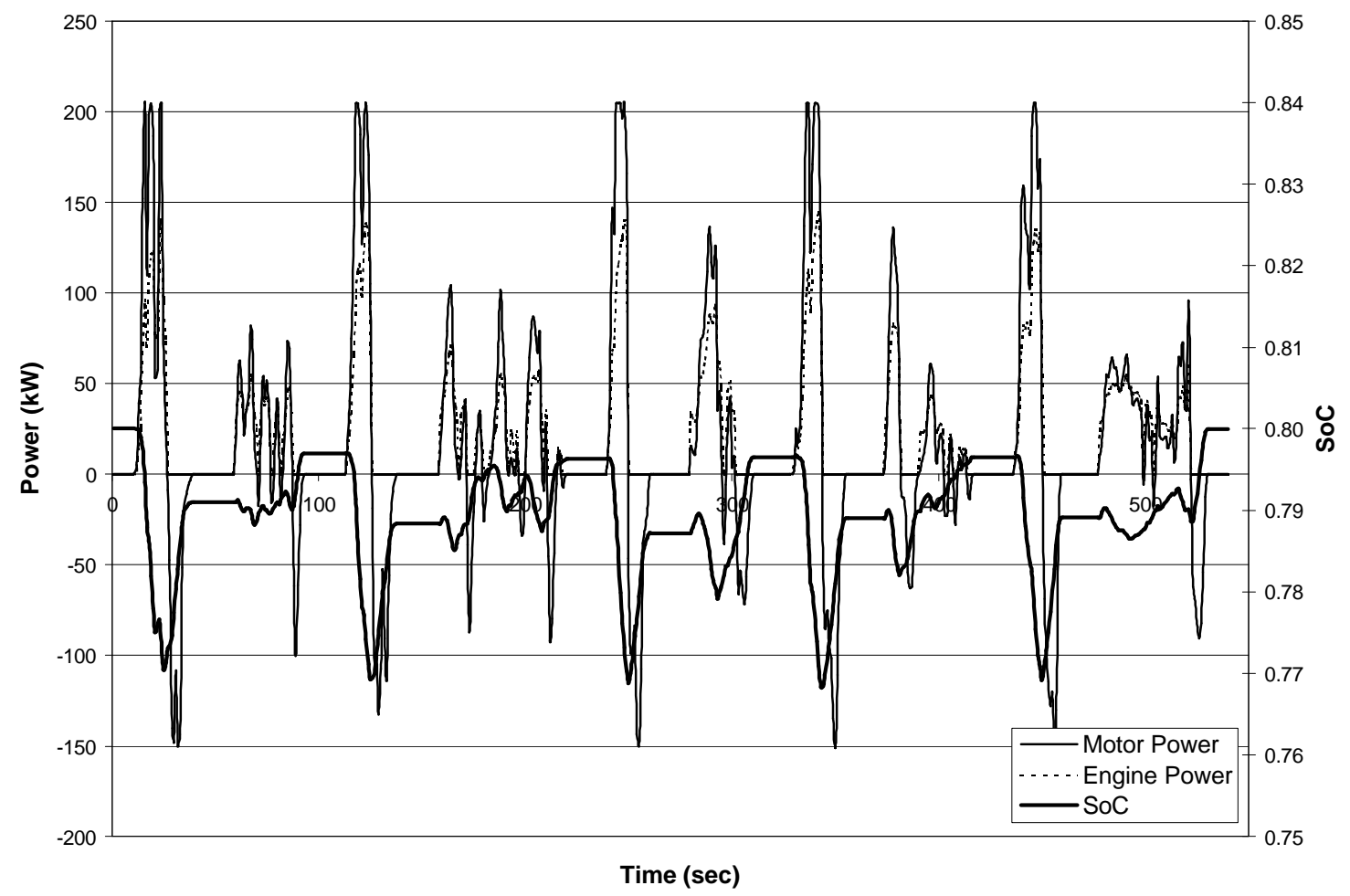

Figure A.45 Class 8 Series HEV on Manhattan Cycle without auxiliary load.

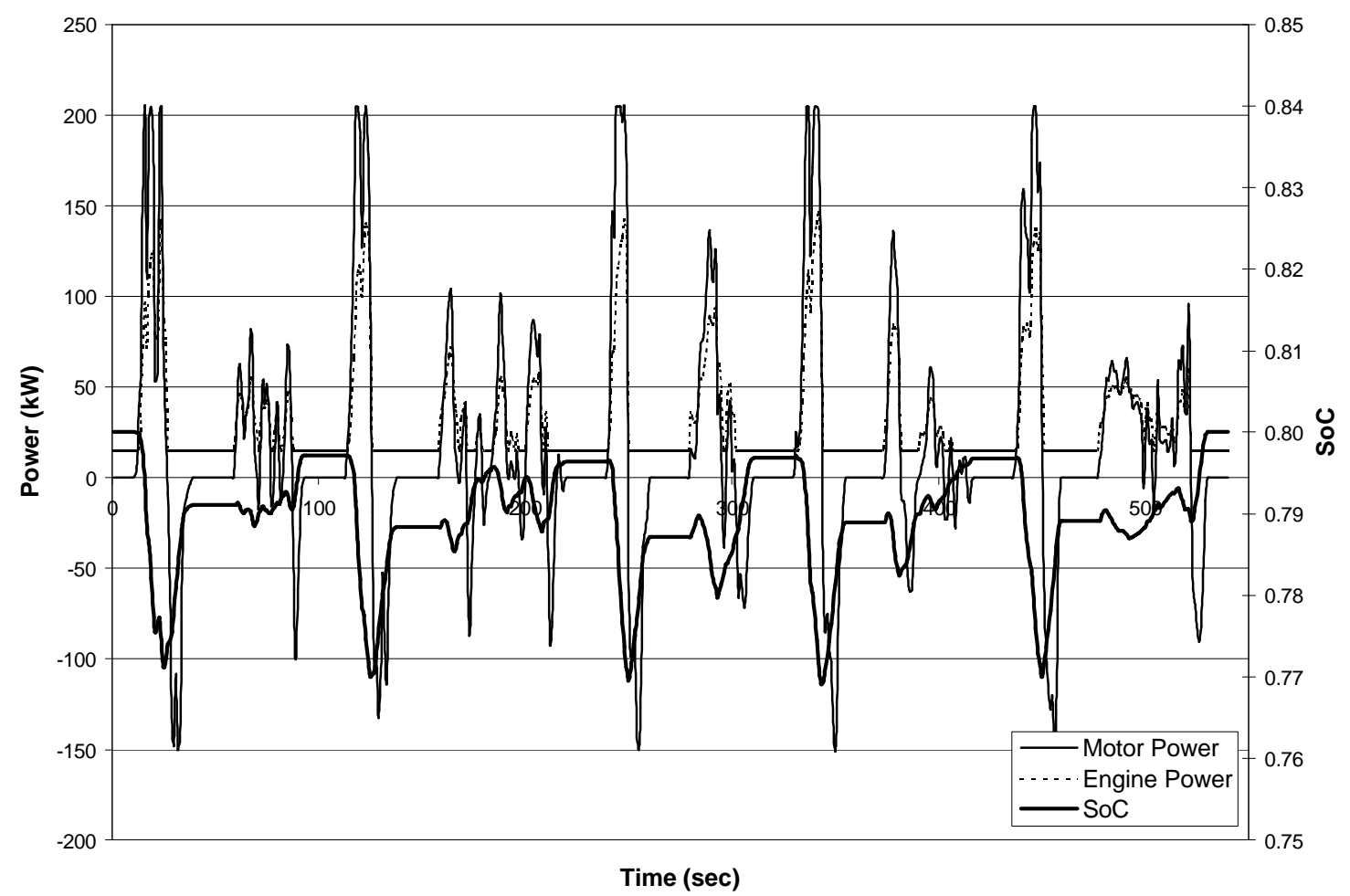

Figure A.46 Class 8 Series HEV on Manhattan Cycle with auxiliary load. 


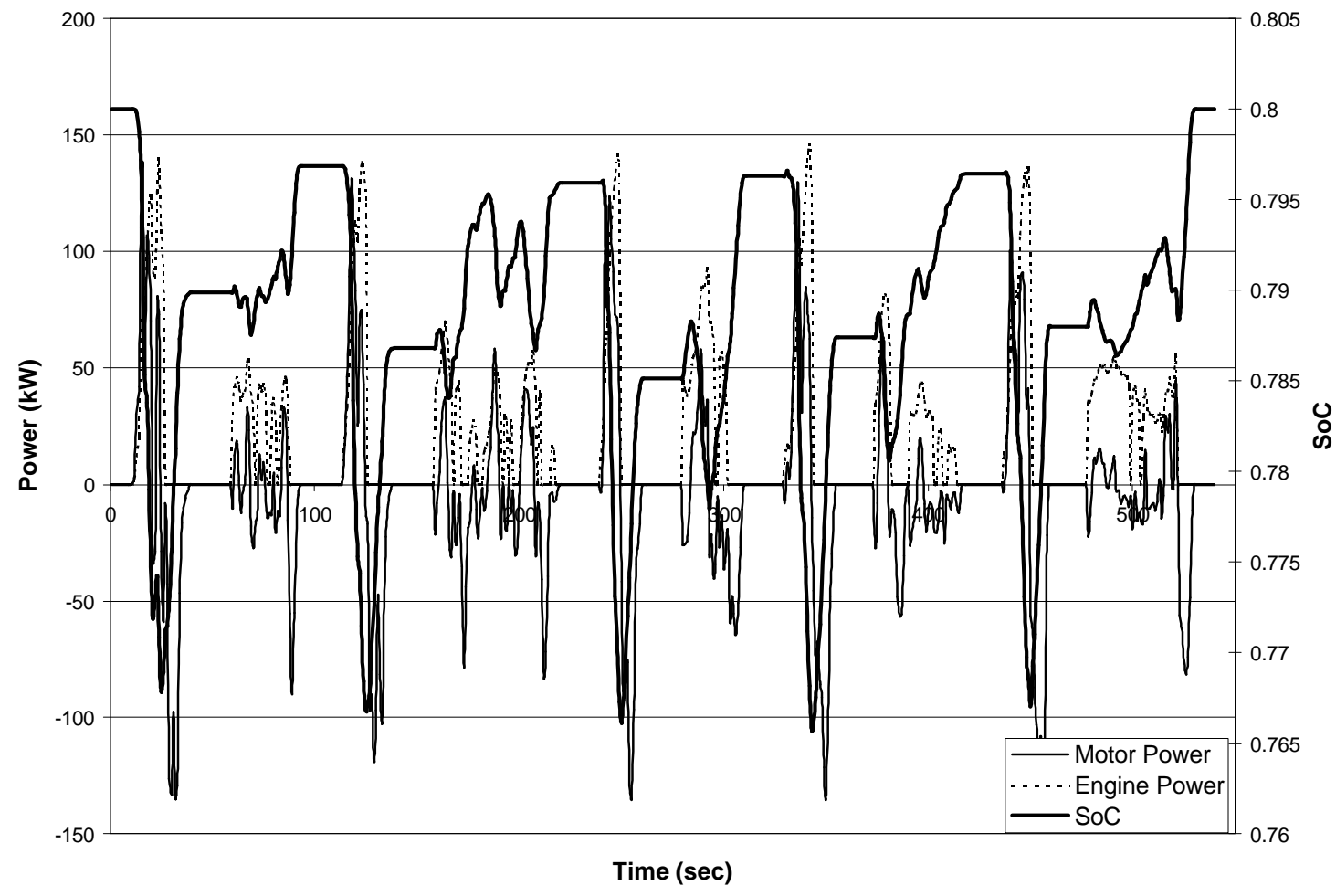

Figure A.47 Class 8 Parallel HEV on Manhattan Cycle without auxiliary load.

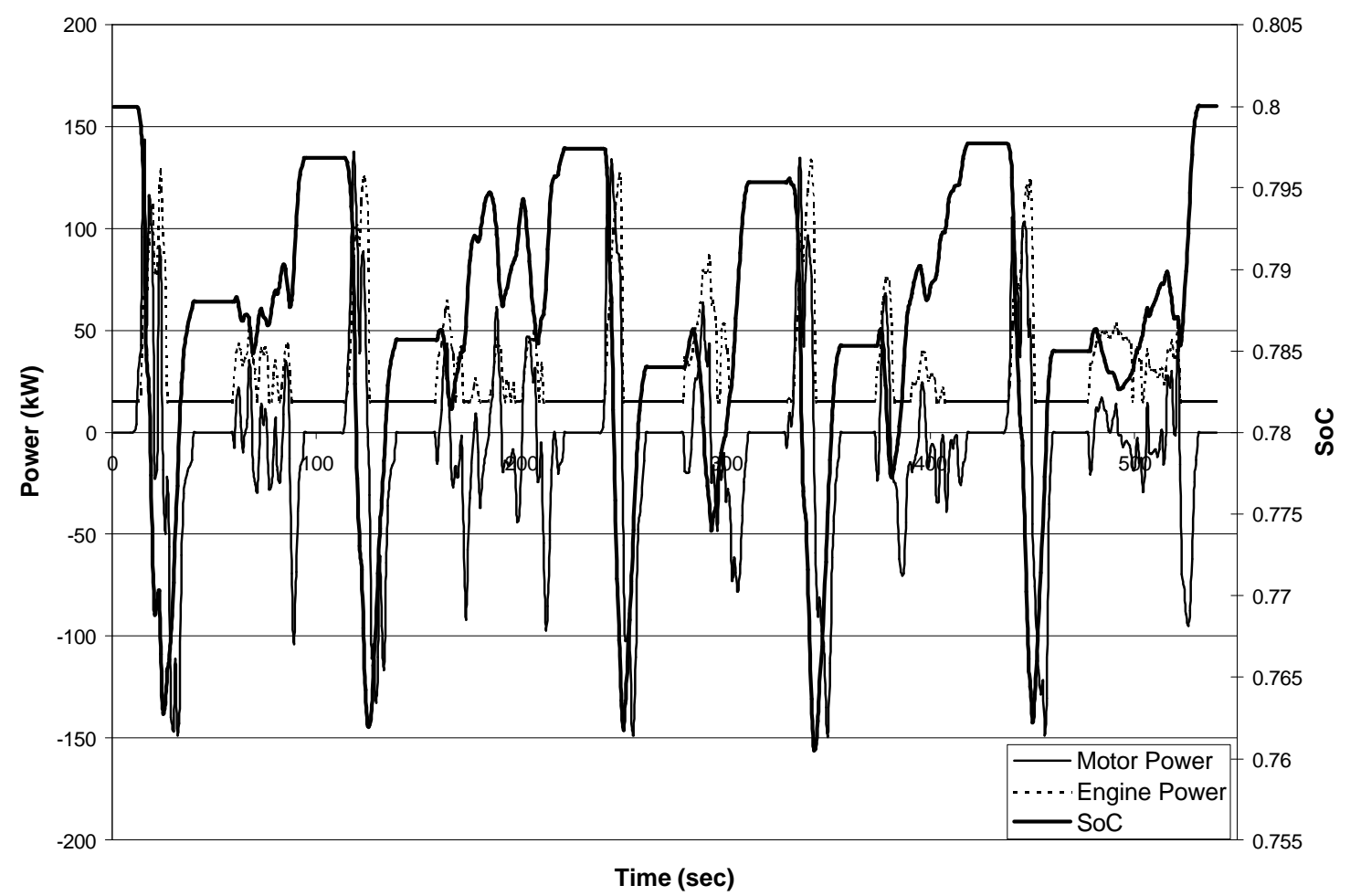

Figure A.48 Class 8 Parallel HEV on Manhattan Cycle with auxiliary load. 


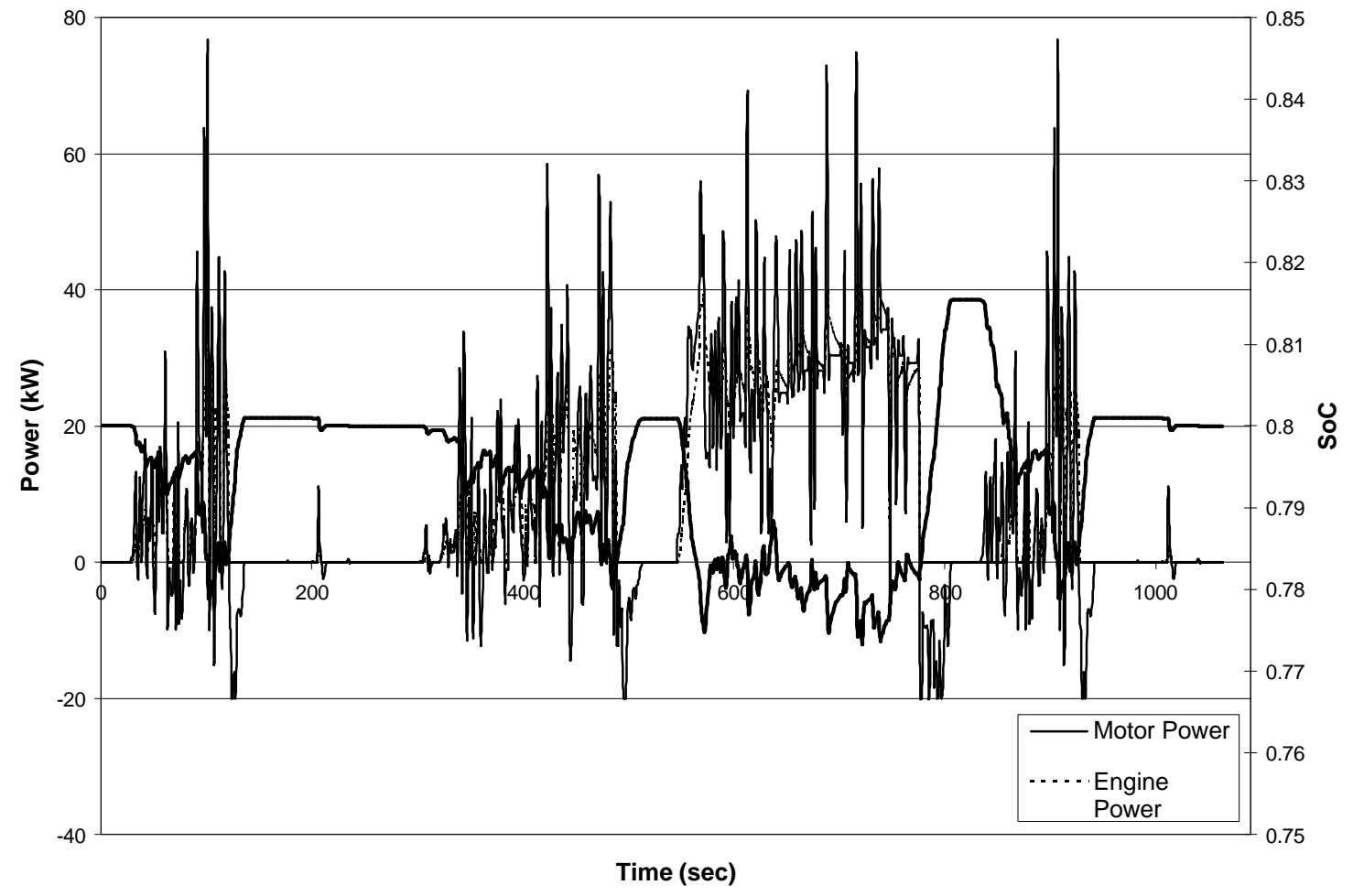

Figure A.49 Class 2B Series HEV on Test D Cycle without auxiliary load.

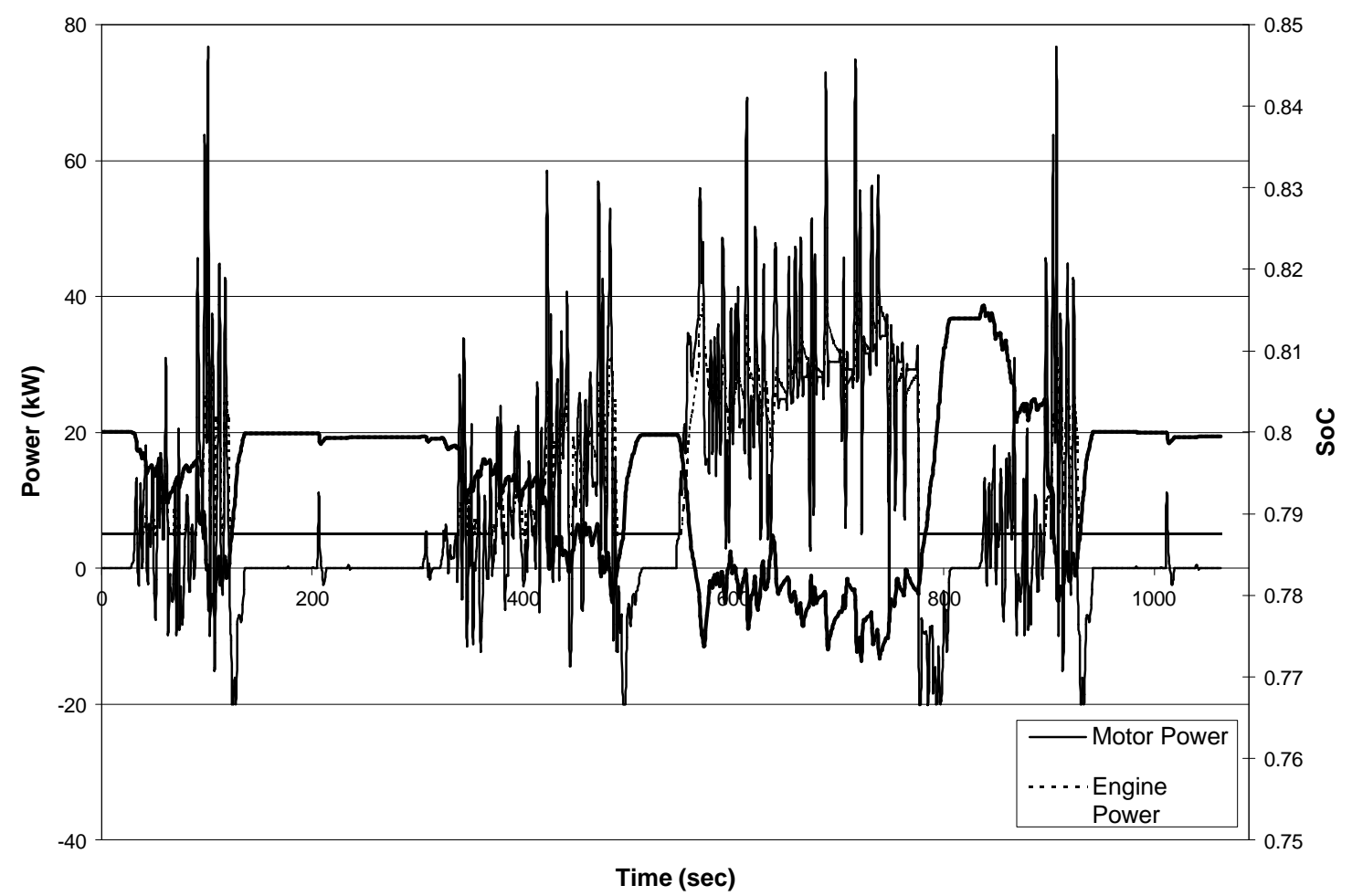

Figure A.50 Class 2B Series HEV on Test D Cycle with auxiliary load. 


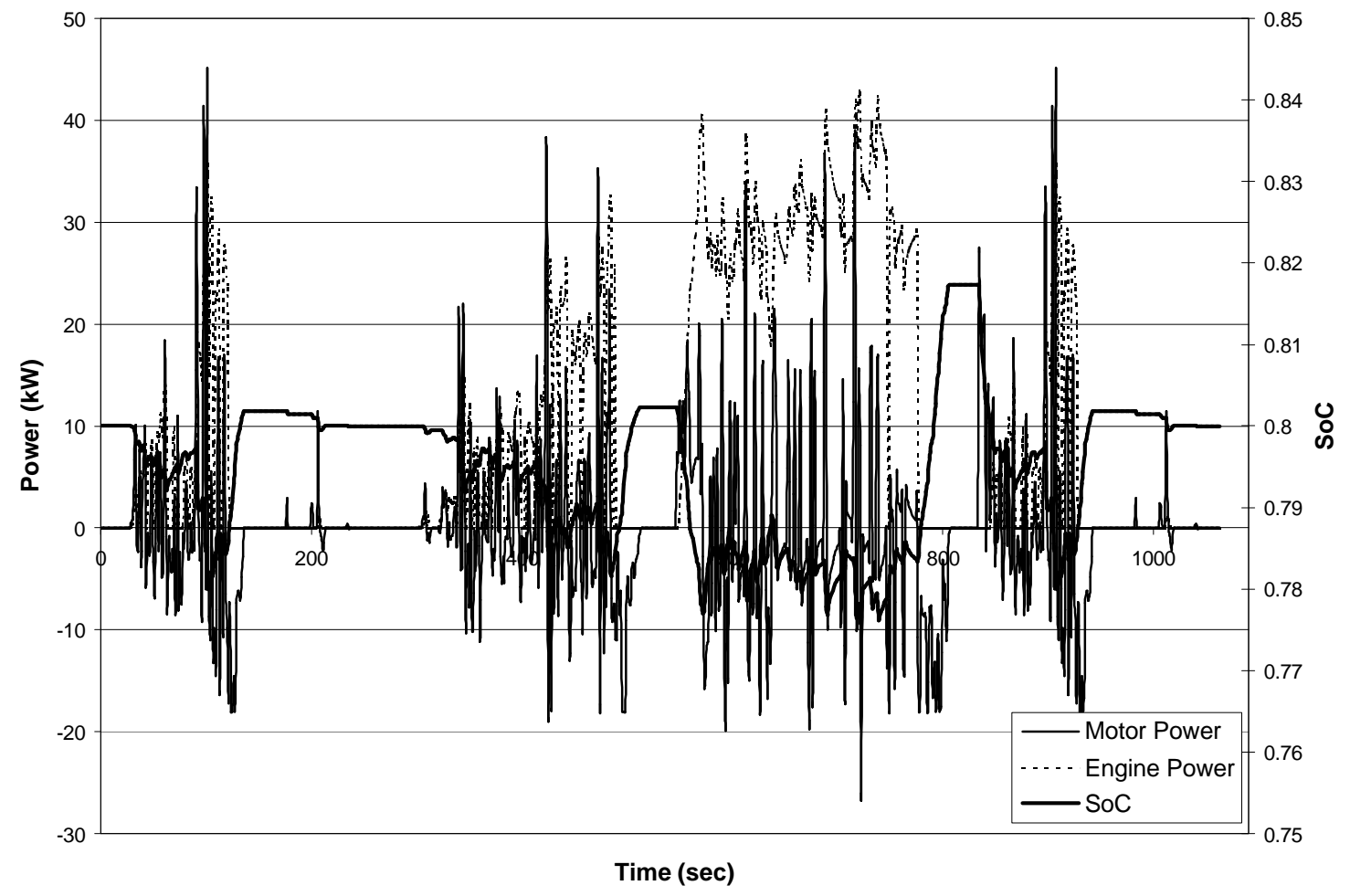

Figure A.51 Class 2B Parallel HEV on Test D Cycle without auxiliary load.

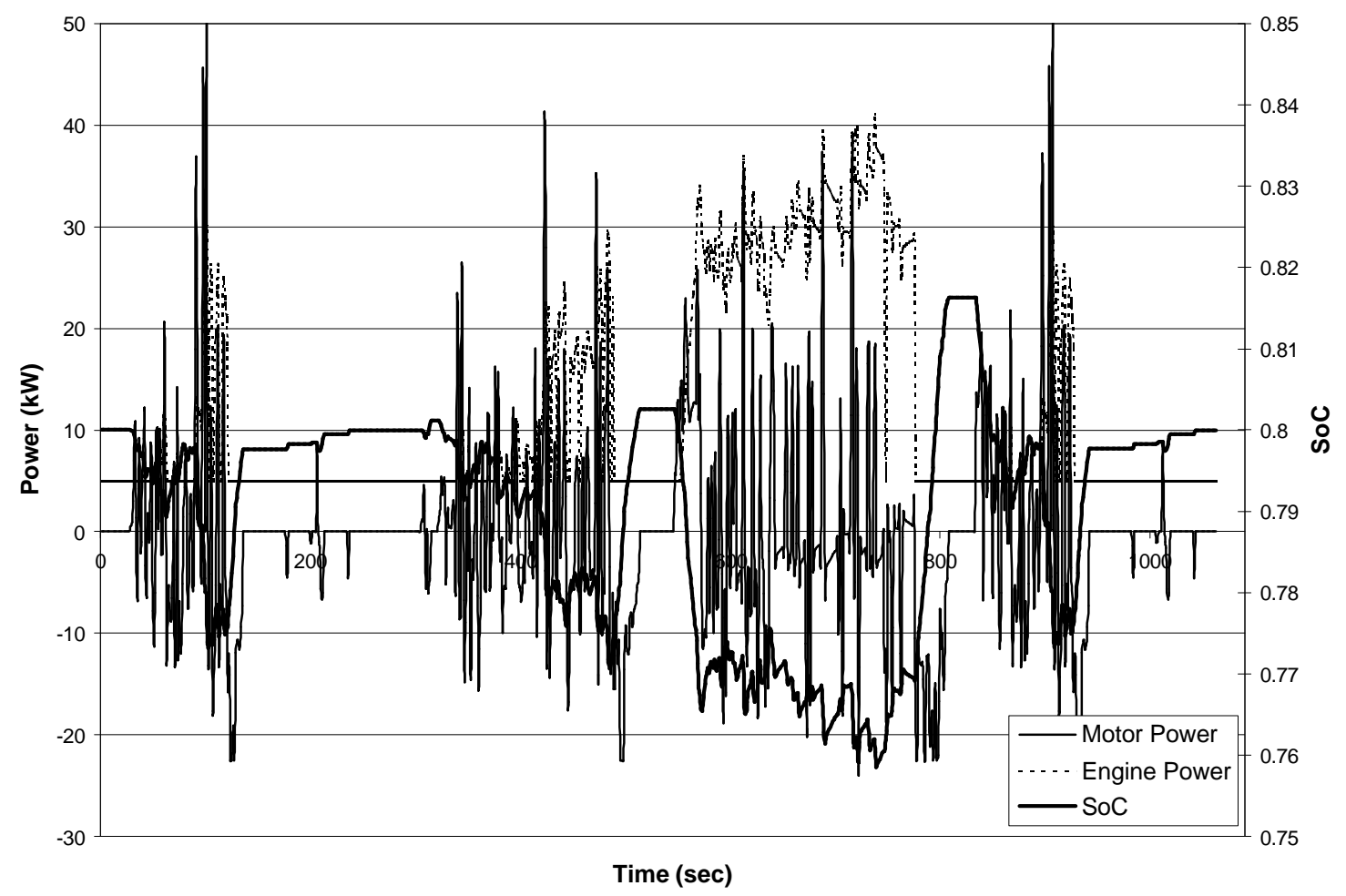

Figure A.52 Class 2B Parallel HEV on Test D Cycle with auxiliary load. 


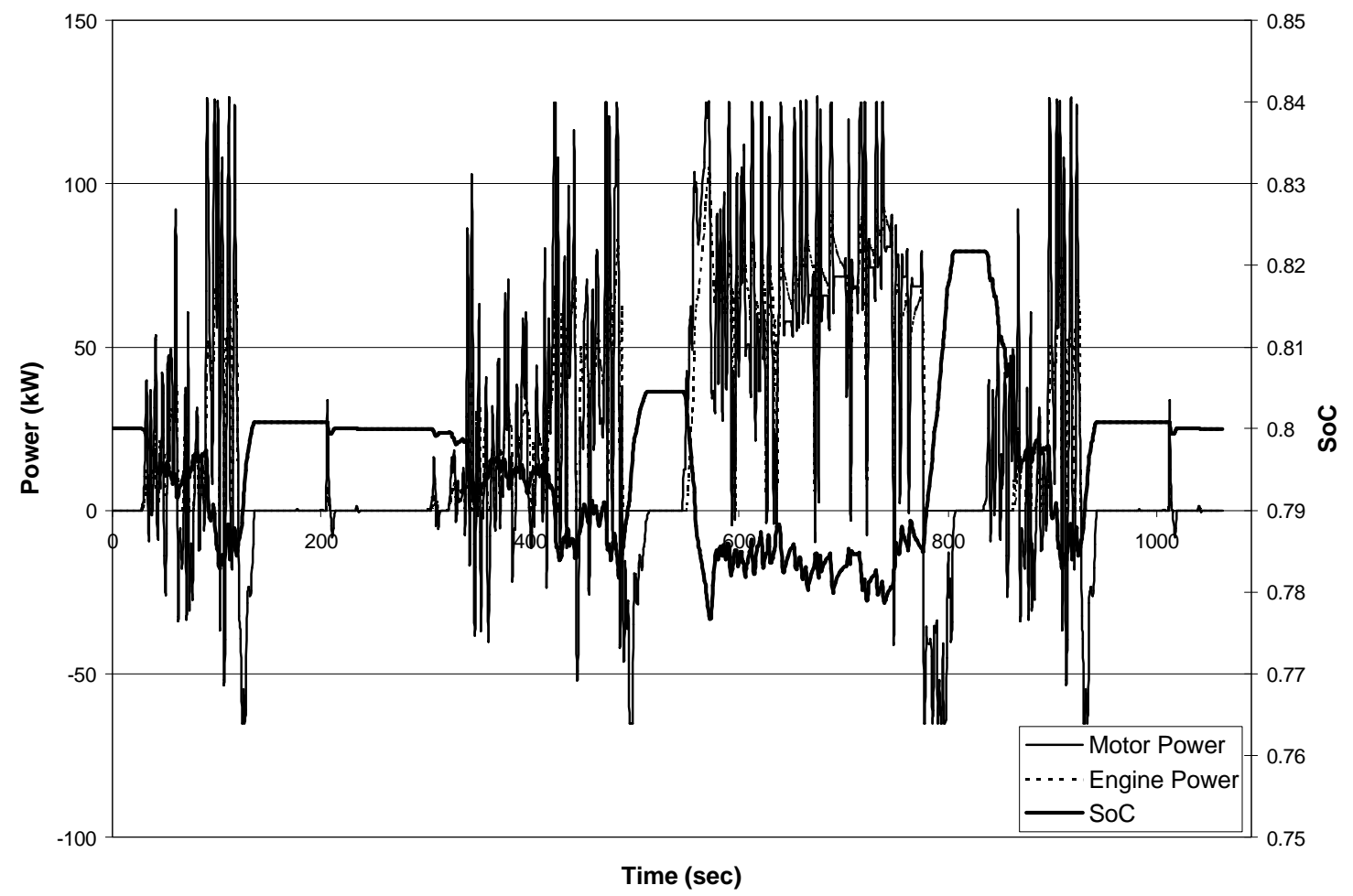

Figure A.53 Class 6 Series HEV on Test D Cycle without auxiliary load.

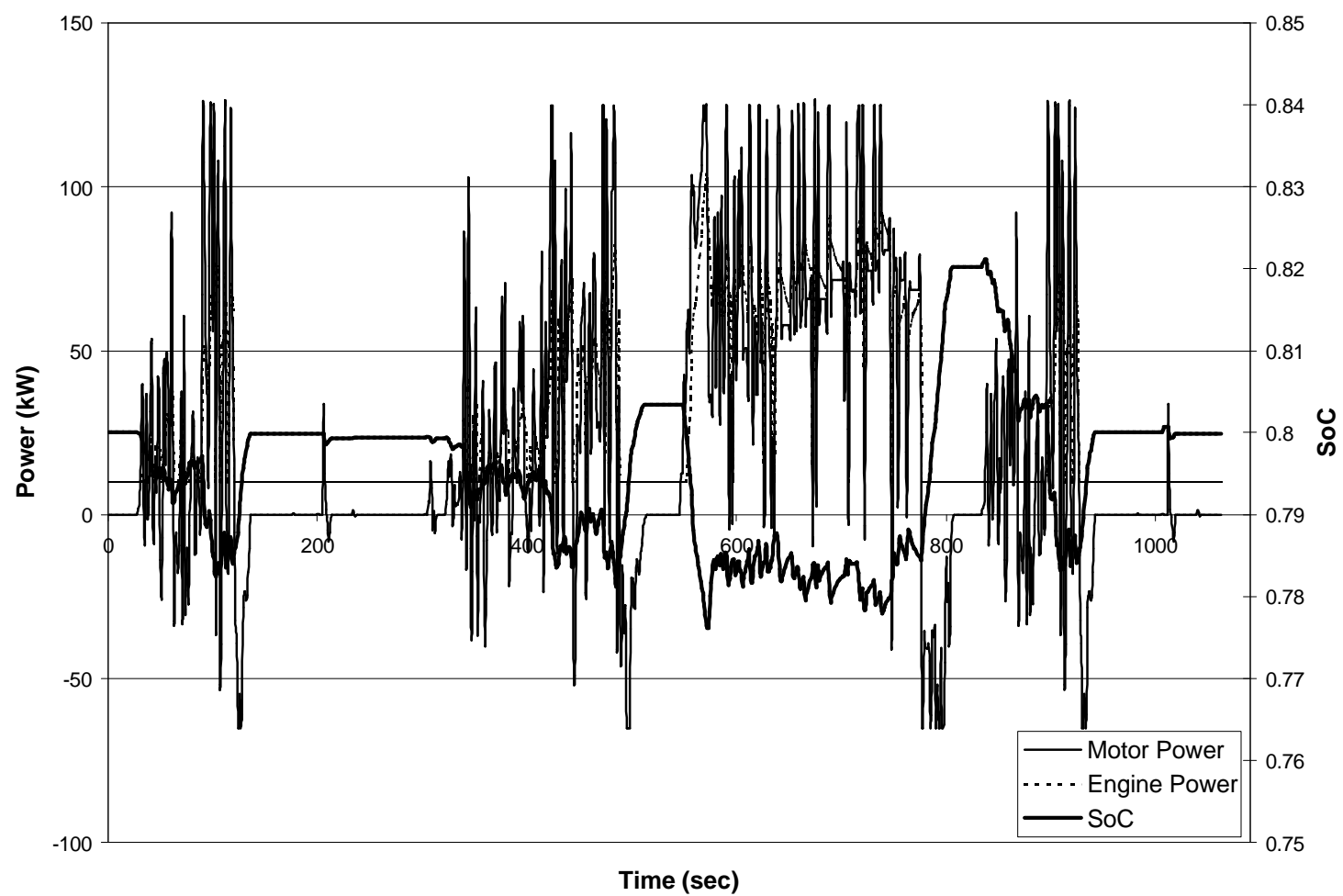

Figure A.54 Class 6 Series HEV on Test D Cycle with auxiliary load. 


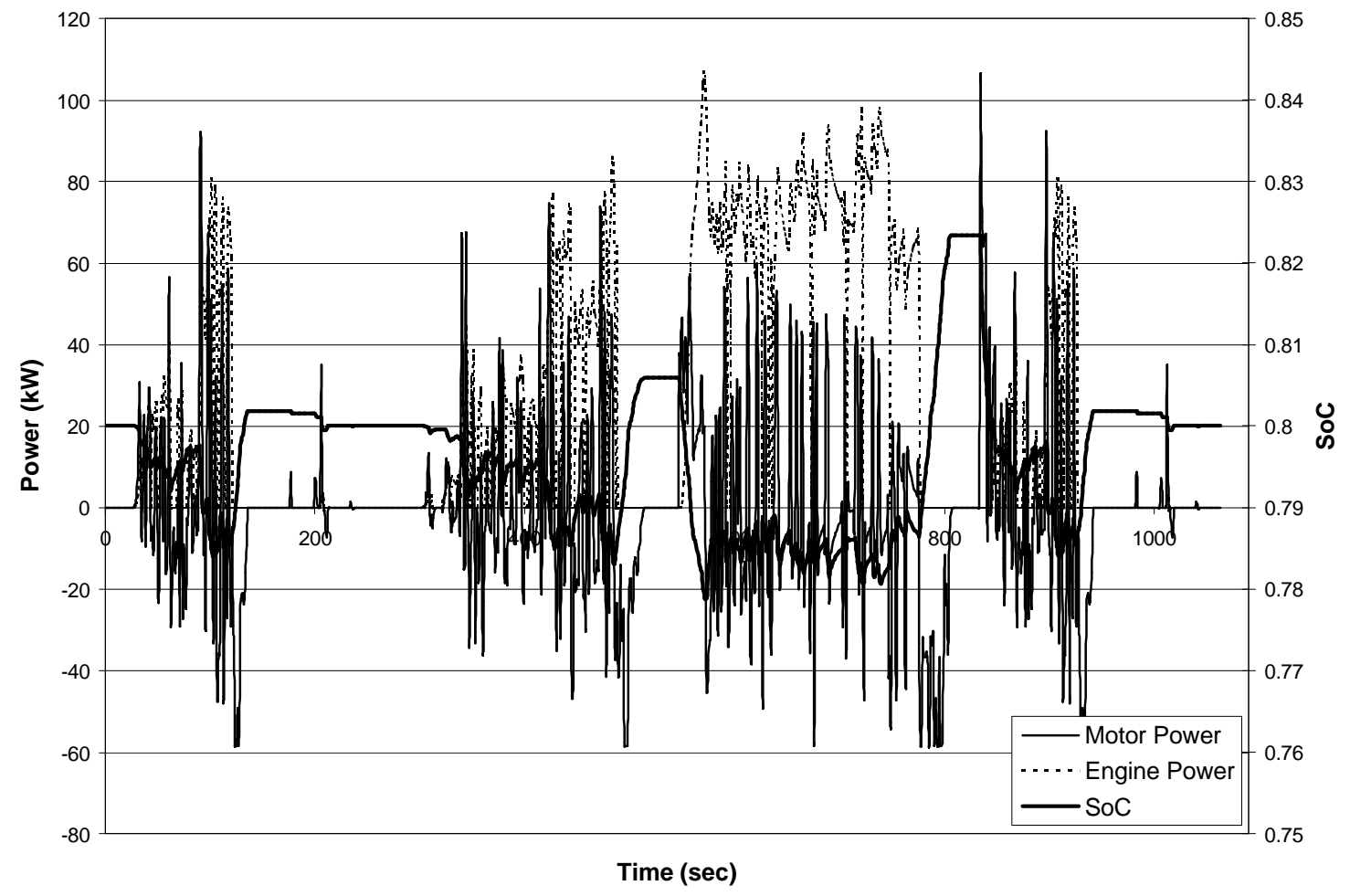

Figure A.55 Class 6 Parallel HEV on Test D Cycle without auxiliary load.

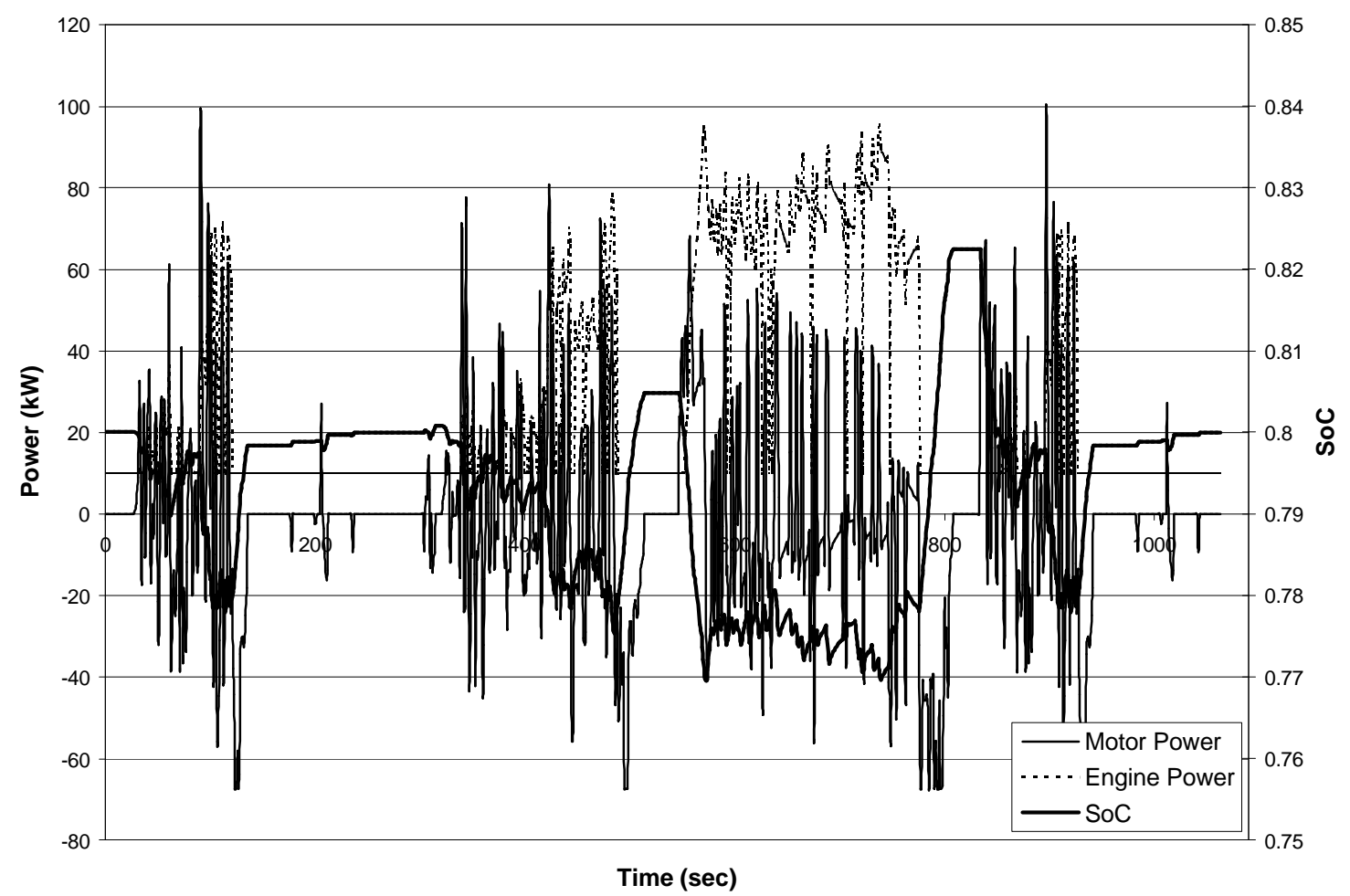

Figure A.56 Class 6 Parallel HEV on Test D Cycle with auxiliary load. 


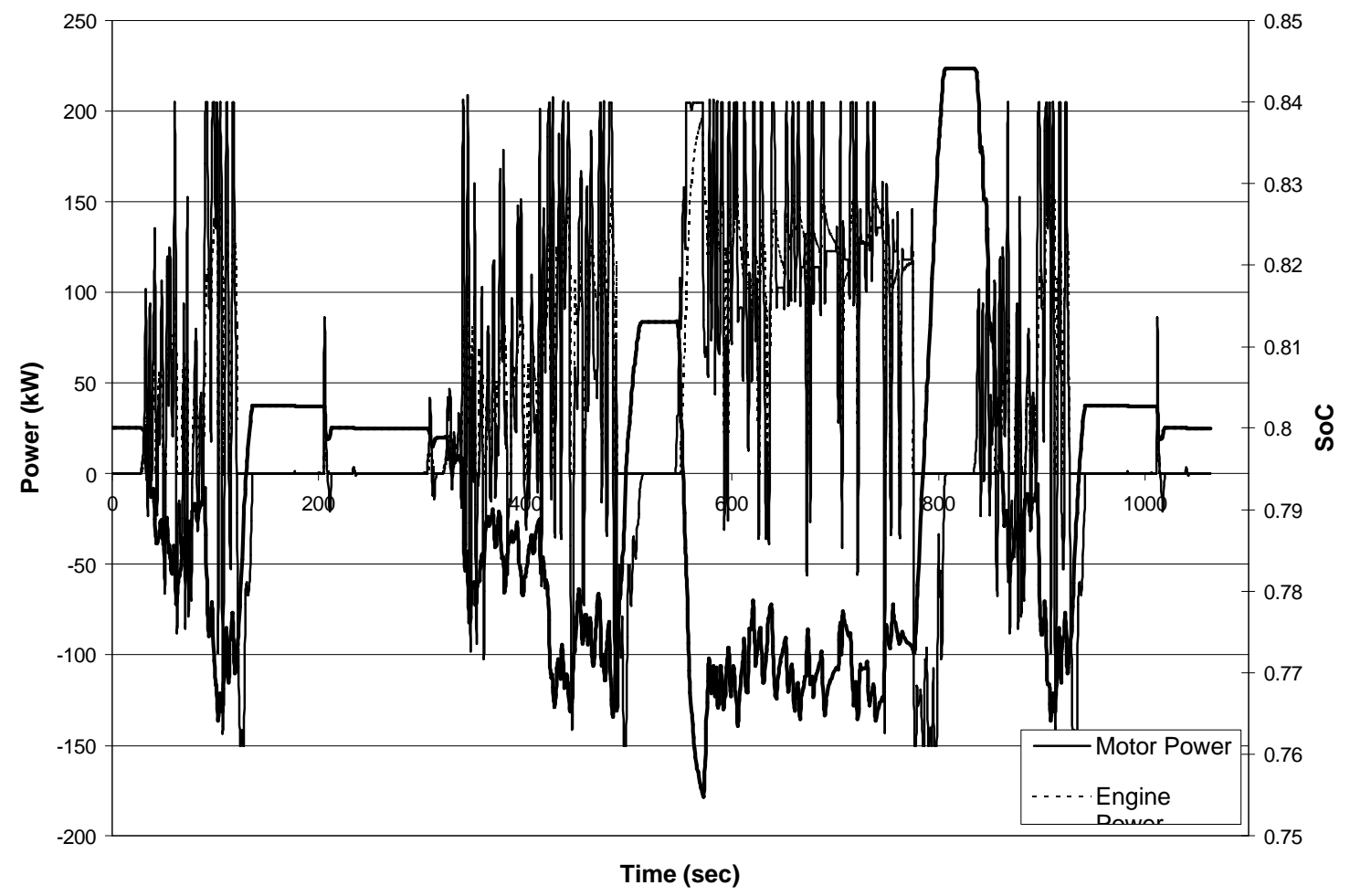

Figure A.57 Class 8 Series HEV on Test D Cycle without auxiliary load.

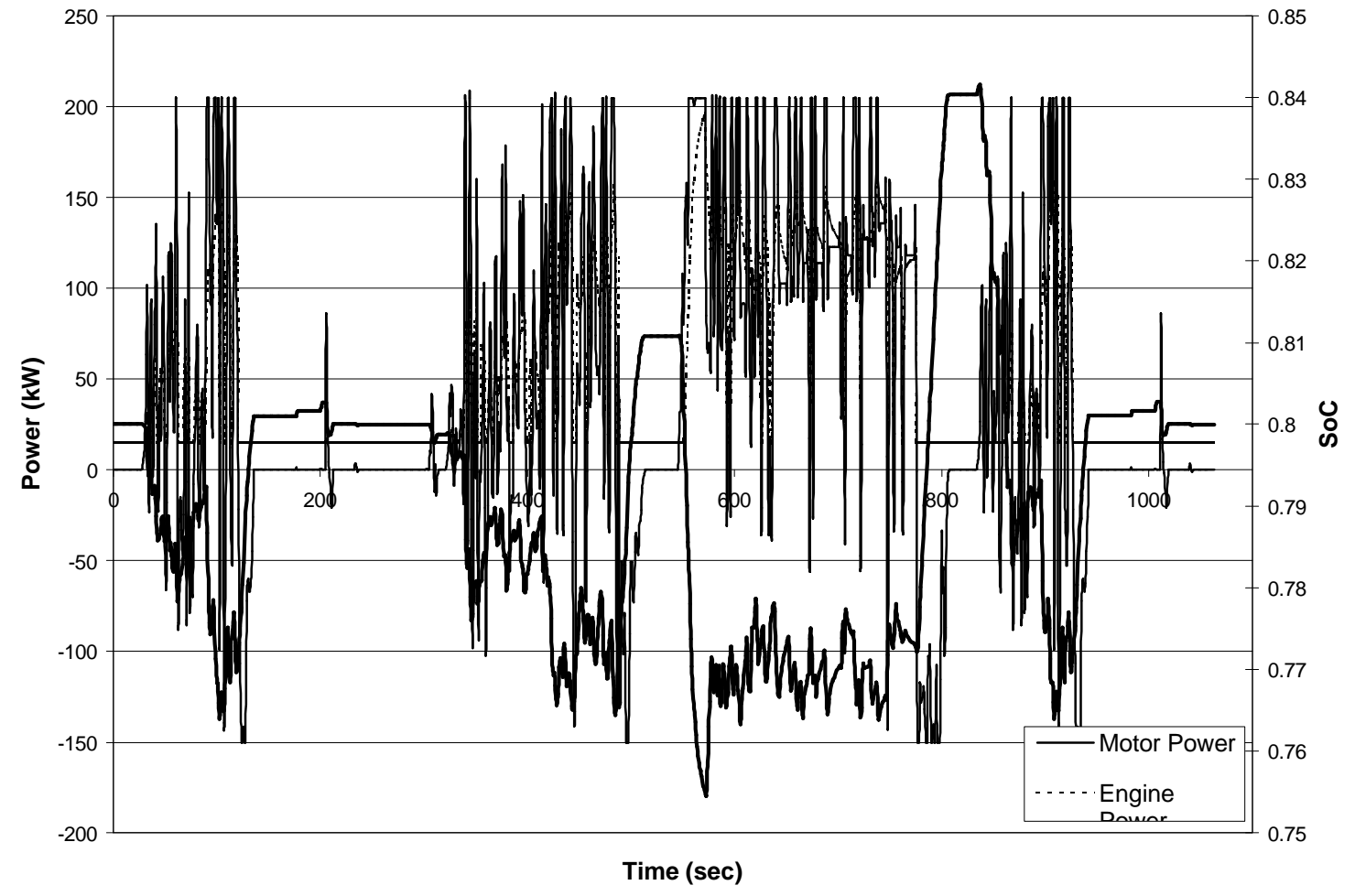

Figure A.58 Class 8 Series HEV on Test D Cycle with auxiliary load. 


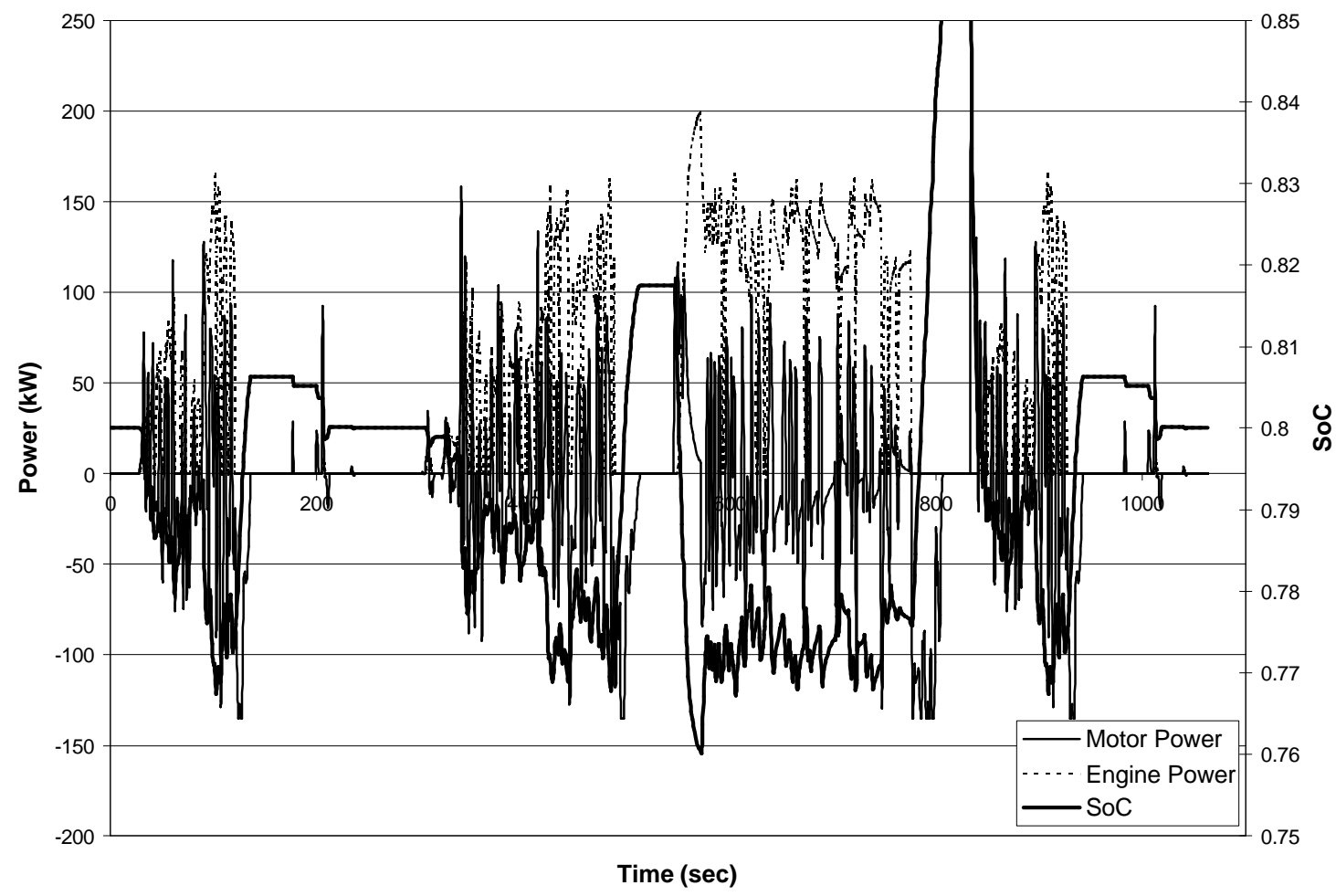

Figure A.59 Class 8 Parallel HEV on Test D Cycle without auxiliary load.

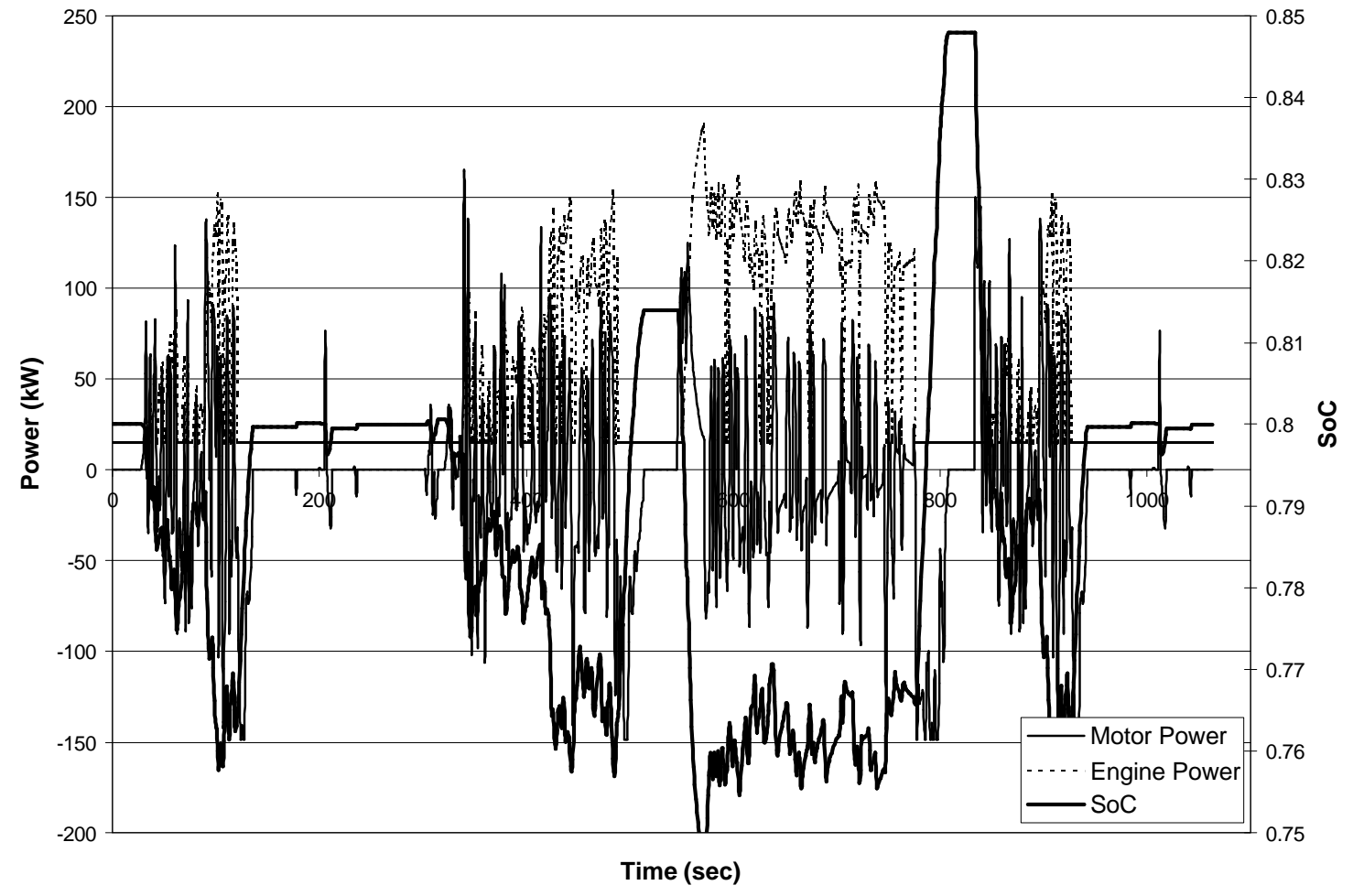

Figure A.60 Class 8 Parallel HEV on Test D Cycle with auxiliary load. 


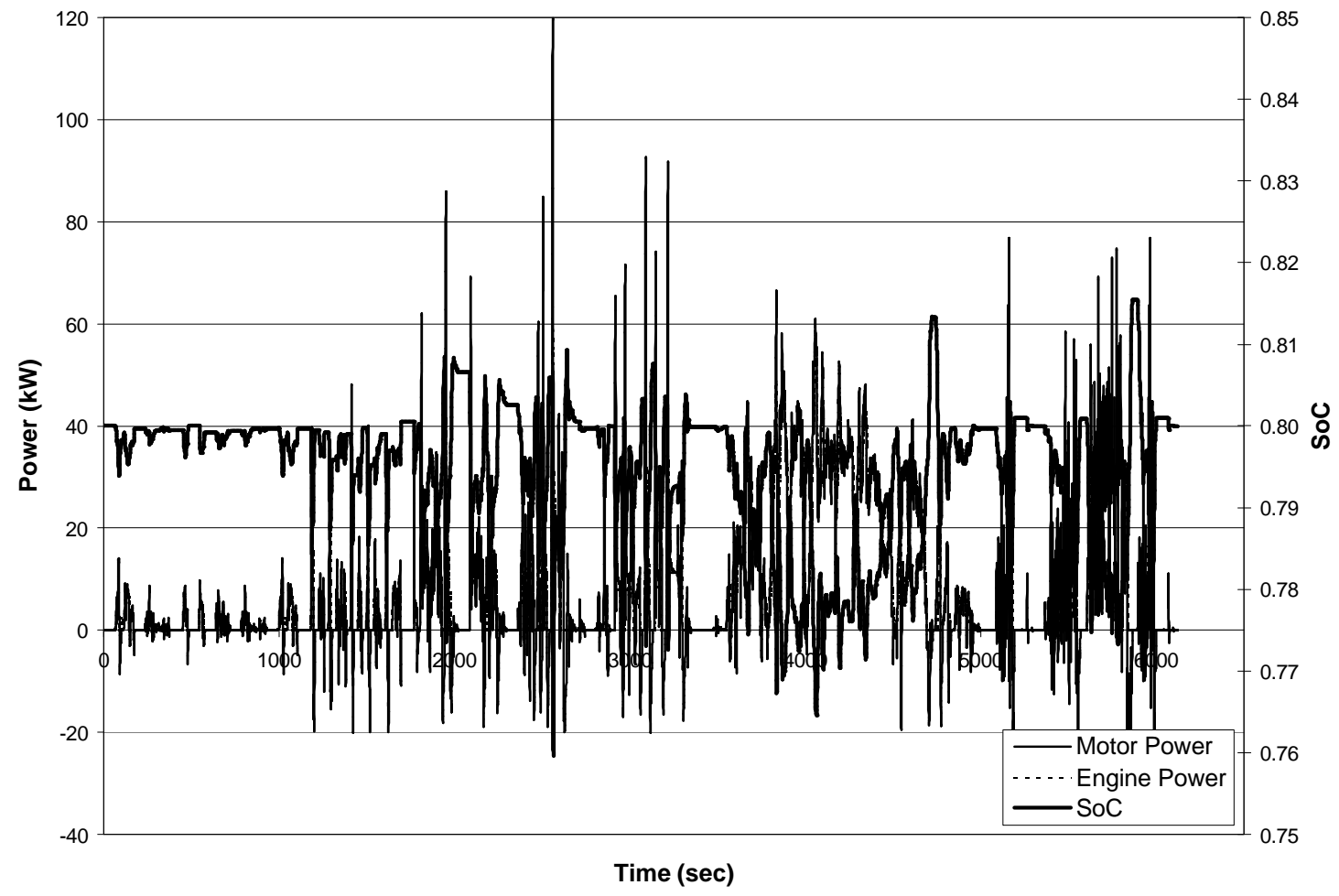

Figure A.61 Class 2B Series HEV on Combined Cycle without auxiliary load.

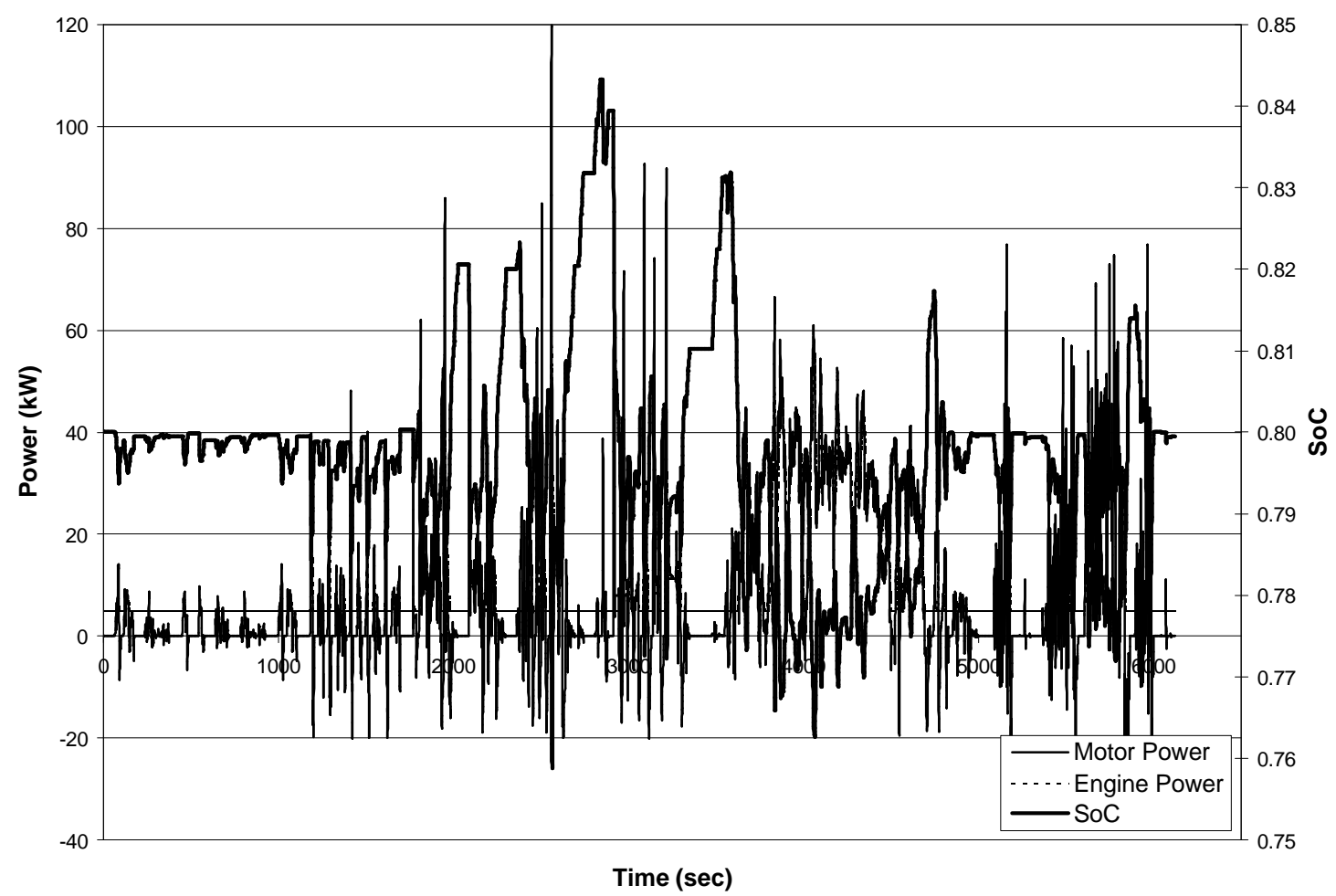

Figure A.62 Class 2B Series HEV on Combined Cycle with auxiliary load. 


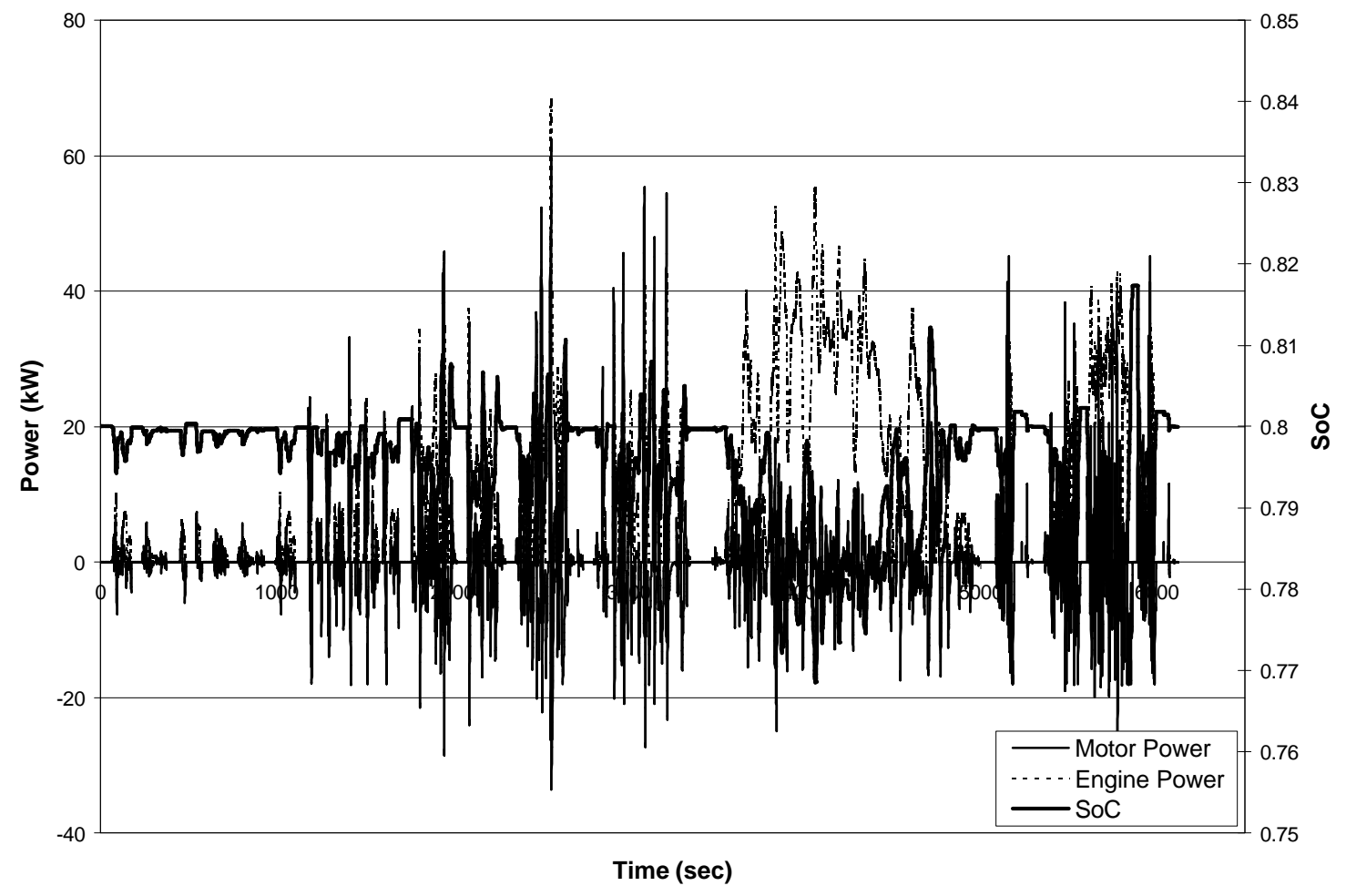

Figure A.63 Class 2B Parallel HEV on Combined Cycle without auxiliary load.

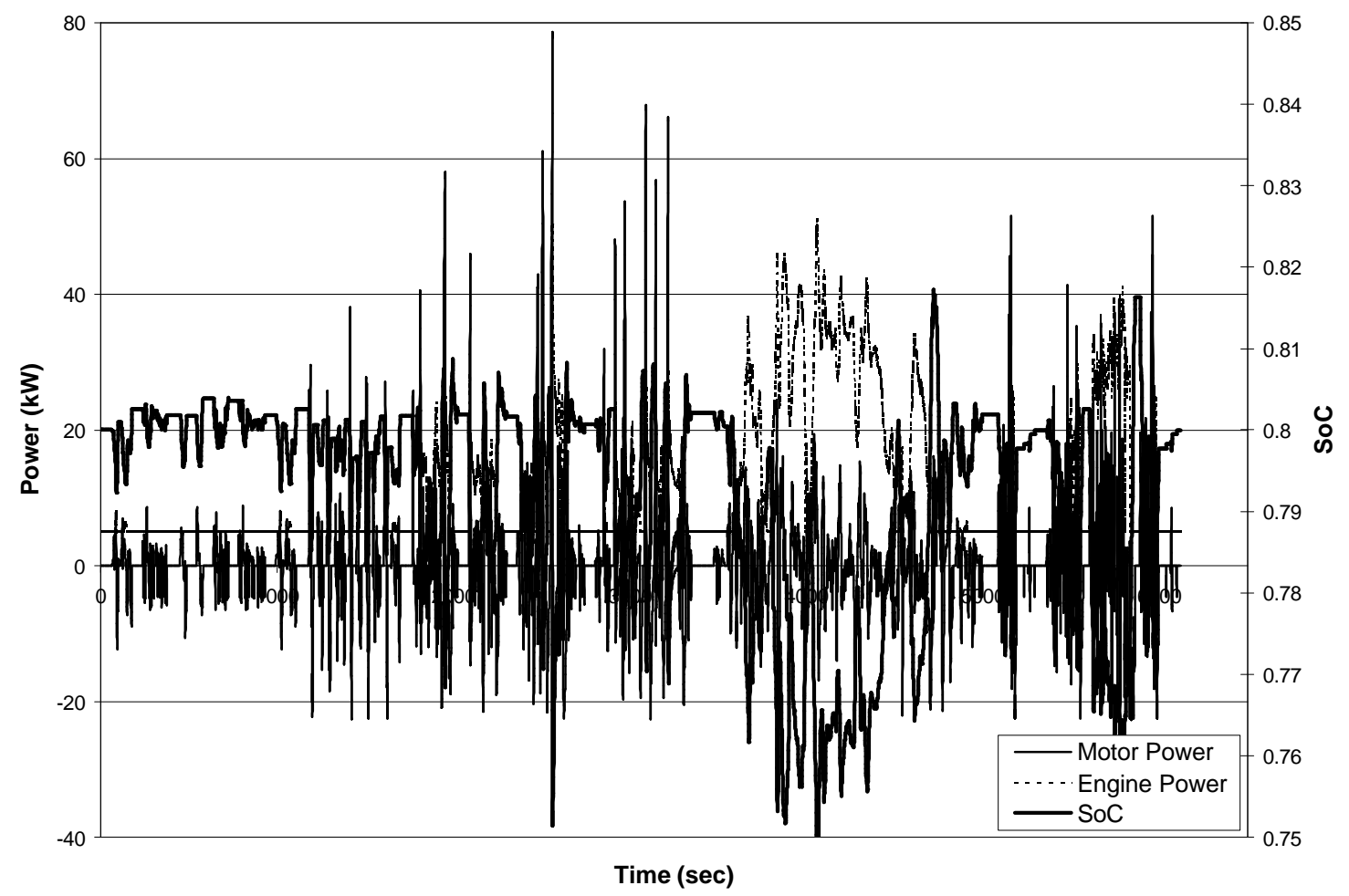

Figure A.64 Class 2B Parallel HEV on Combined cycle with auxiliary load. 


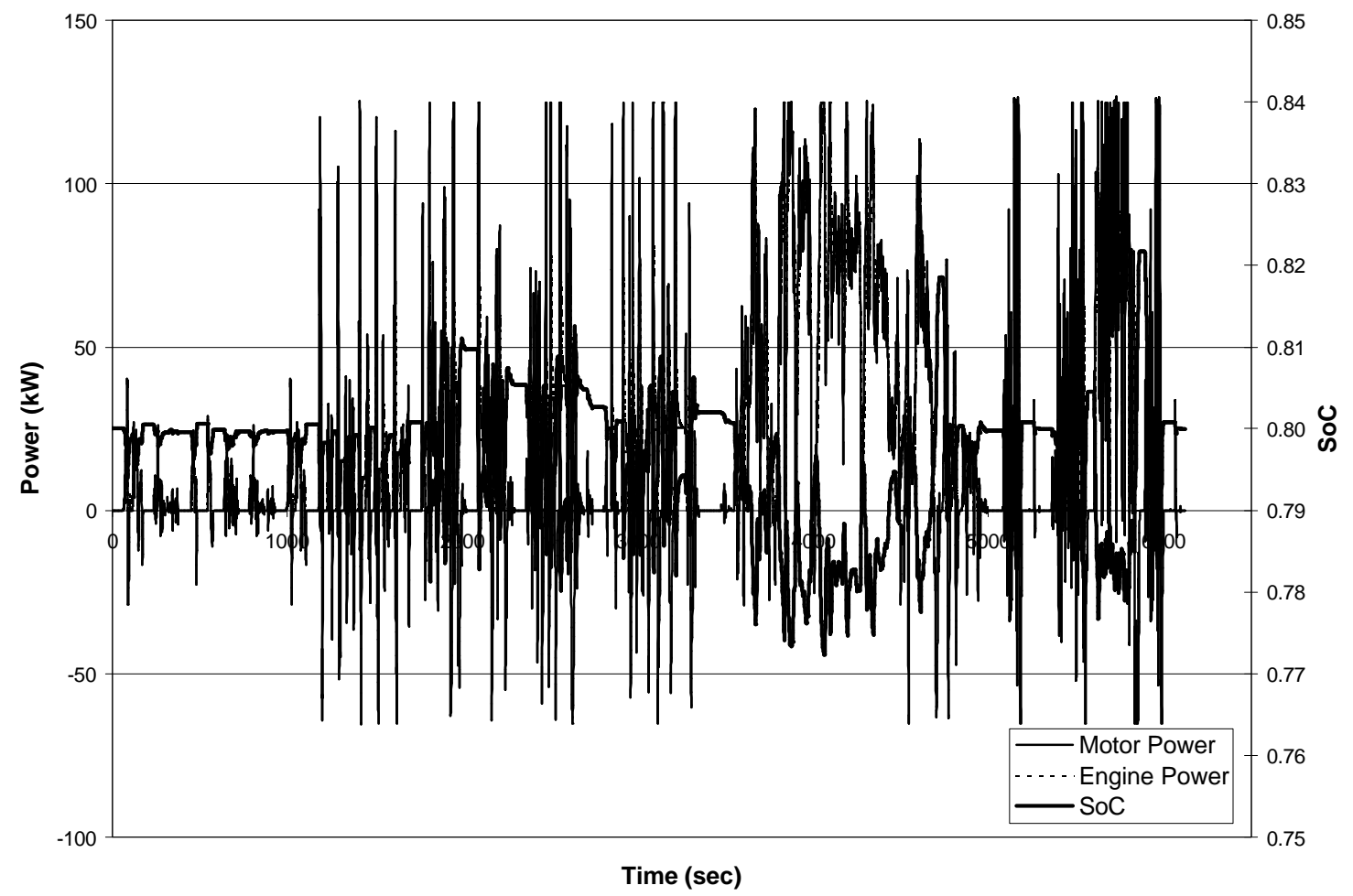

Figure A.65 Class 6 Series HEV on Combined Cycle without auxiliary load.

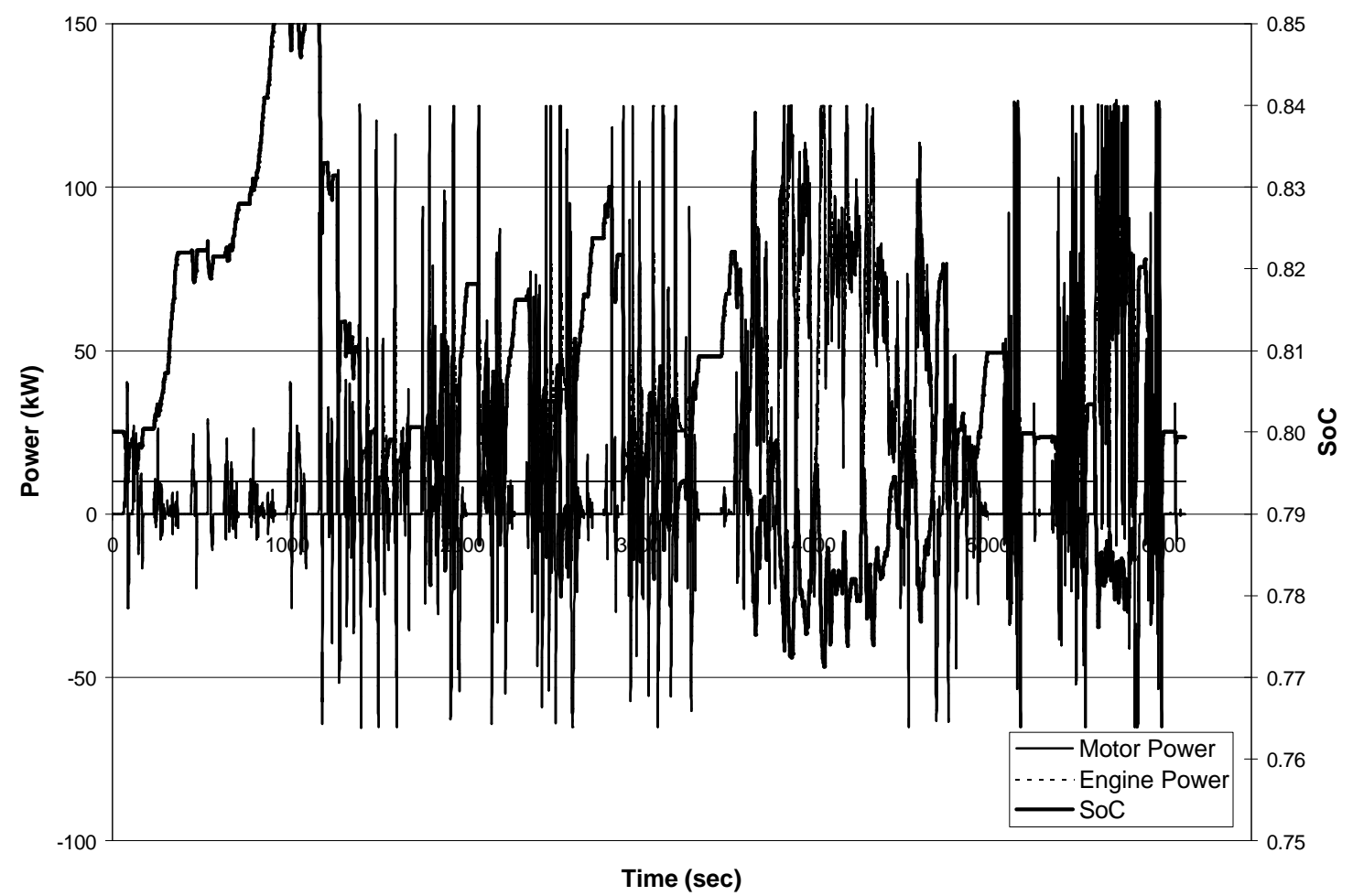

Figure A.66 Class 6 Series HEV on Combined Cycle with auxiliary load. 


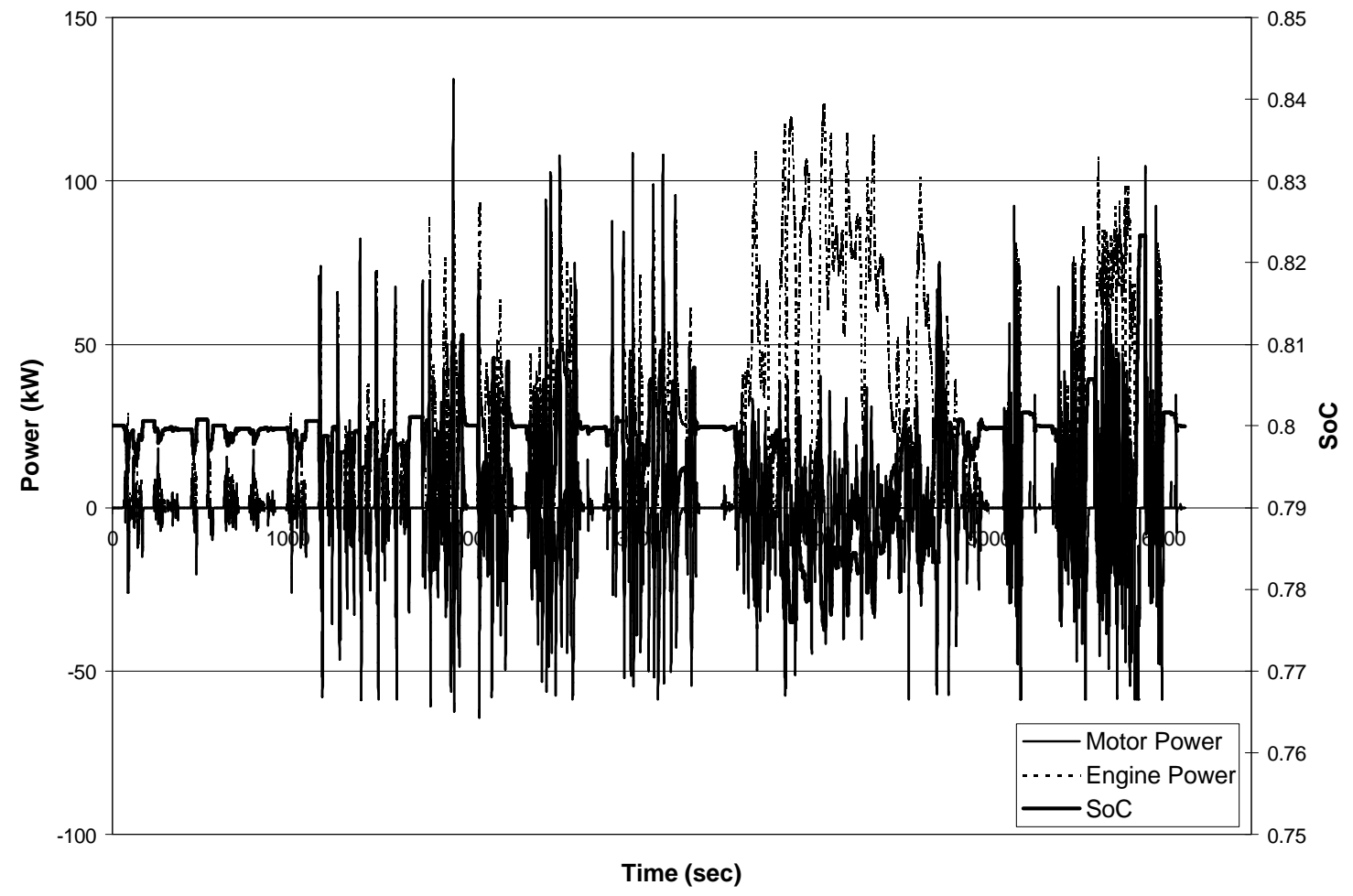

Figure A.67 Class 6 Parallel HEV on Combined Cycle without auxiliary load.

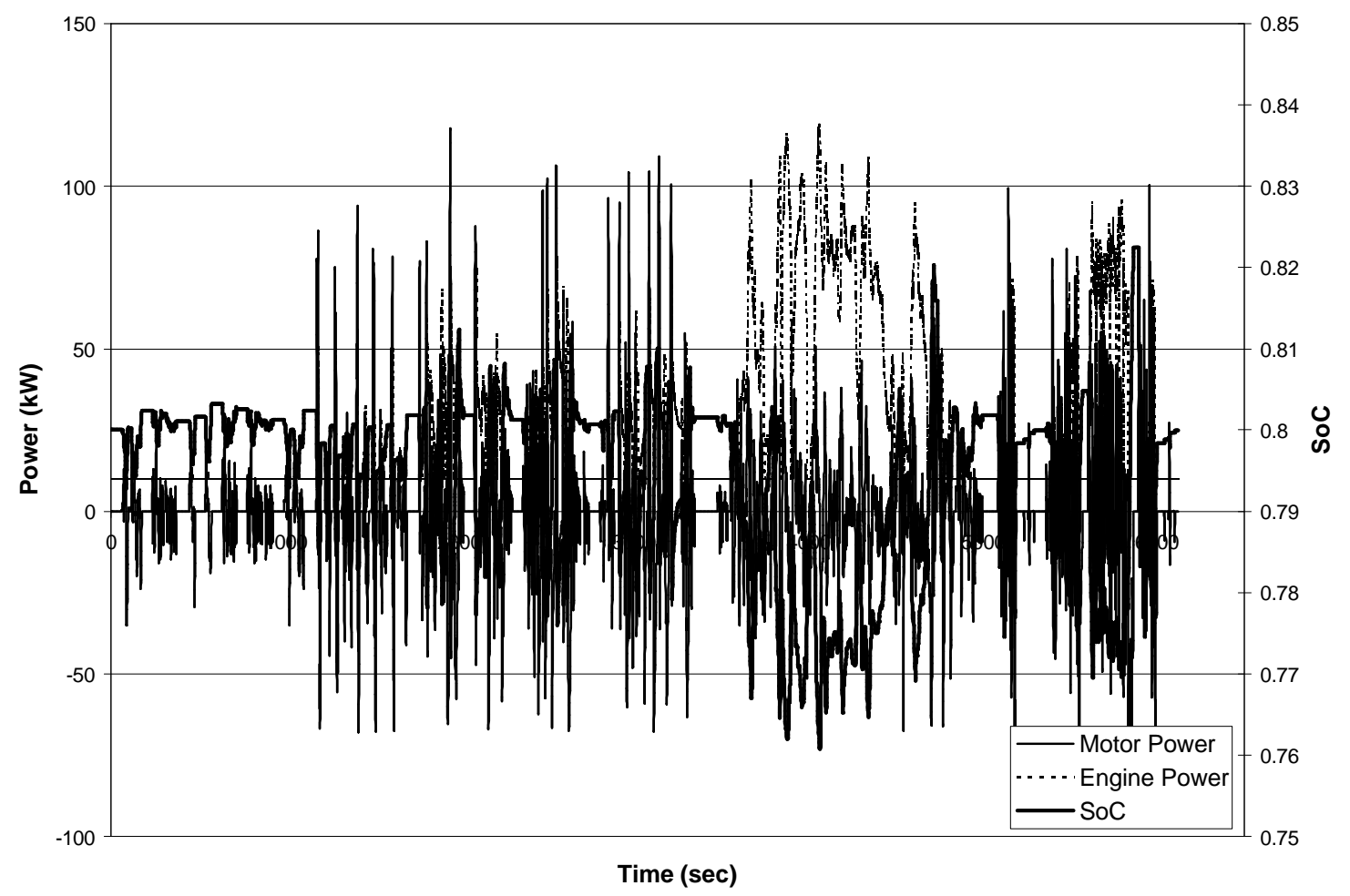

Figure A.68 Class 6 Parallel HEV on Combined Cycle with auxiliary load. 


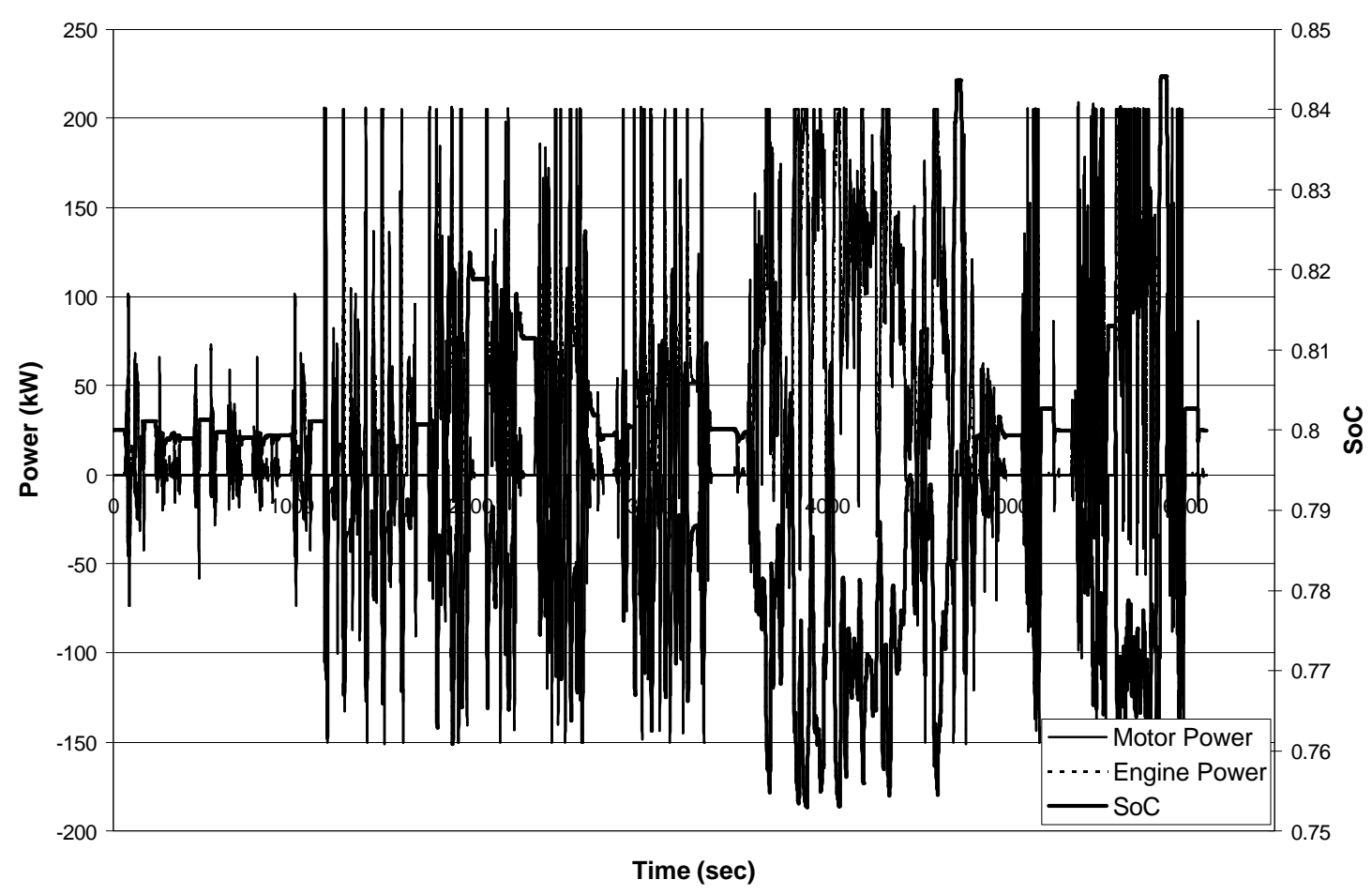

Figure A.69 Class 8 Series HEV on Combined Cycle without auxiliary load.

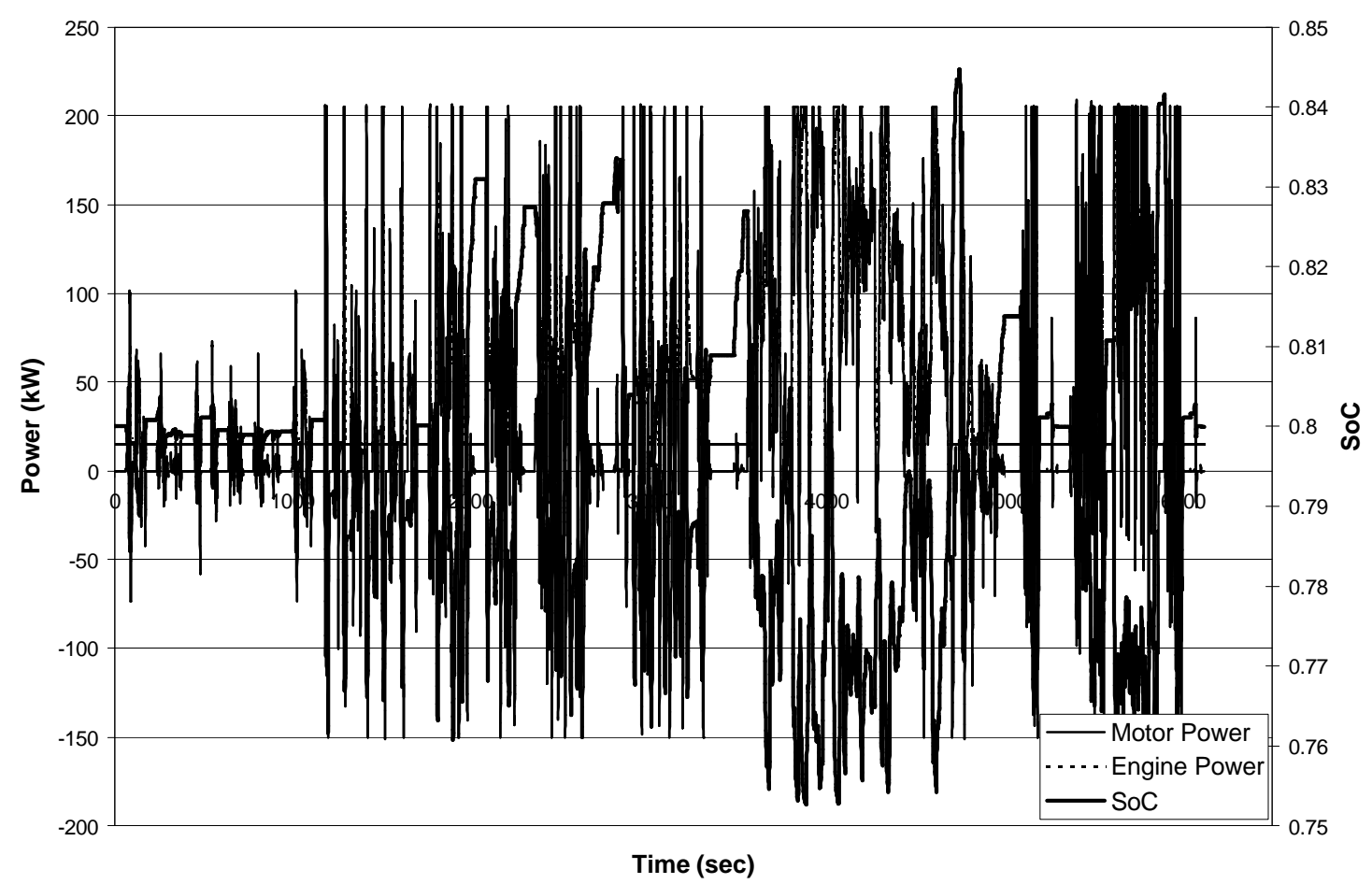

Figure A.70 Class 8 Series HEV on Combined Cycle with auxiliary load. 


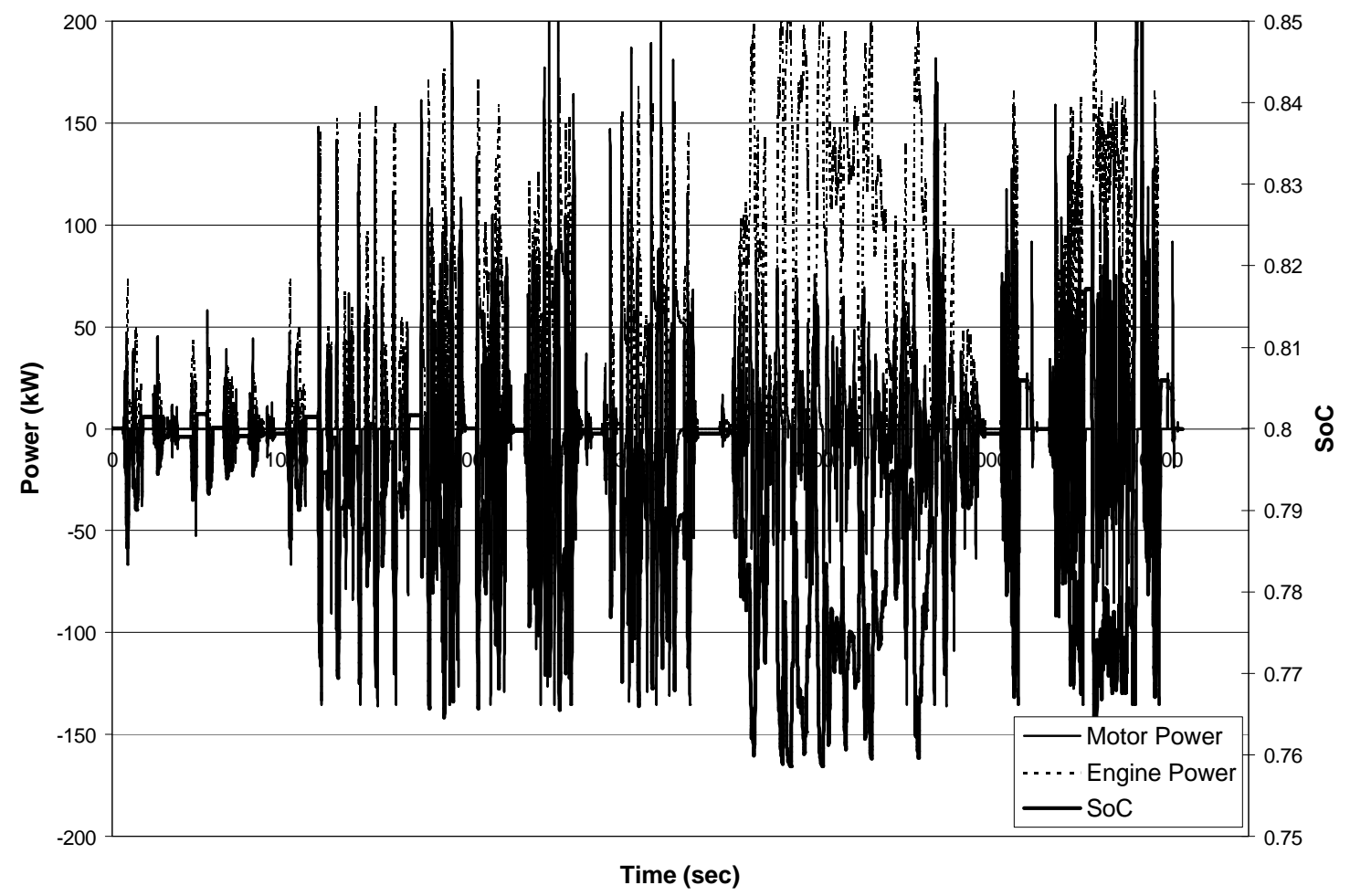

Figure A.71 Class 8 Parallel HEV on Combined Cycle without auxiliary load.

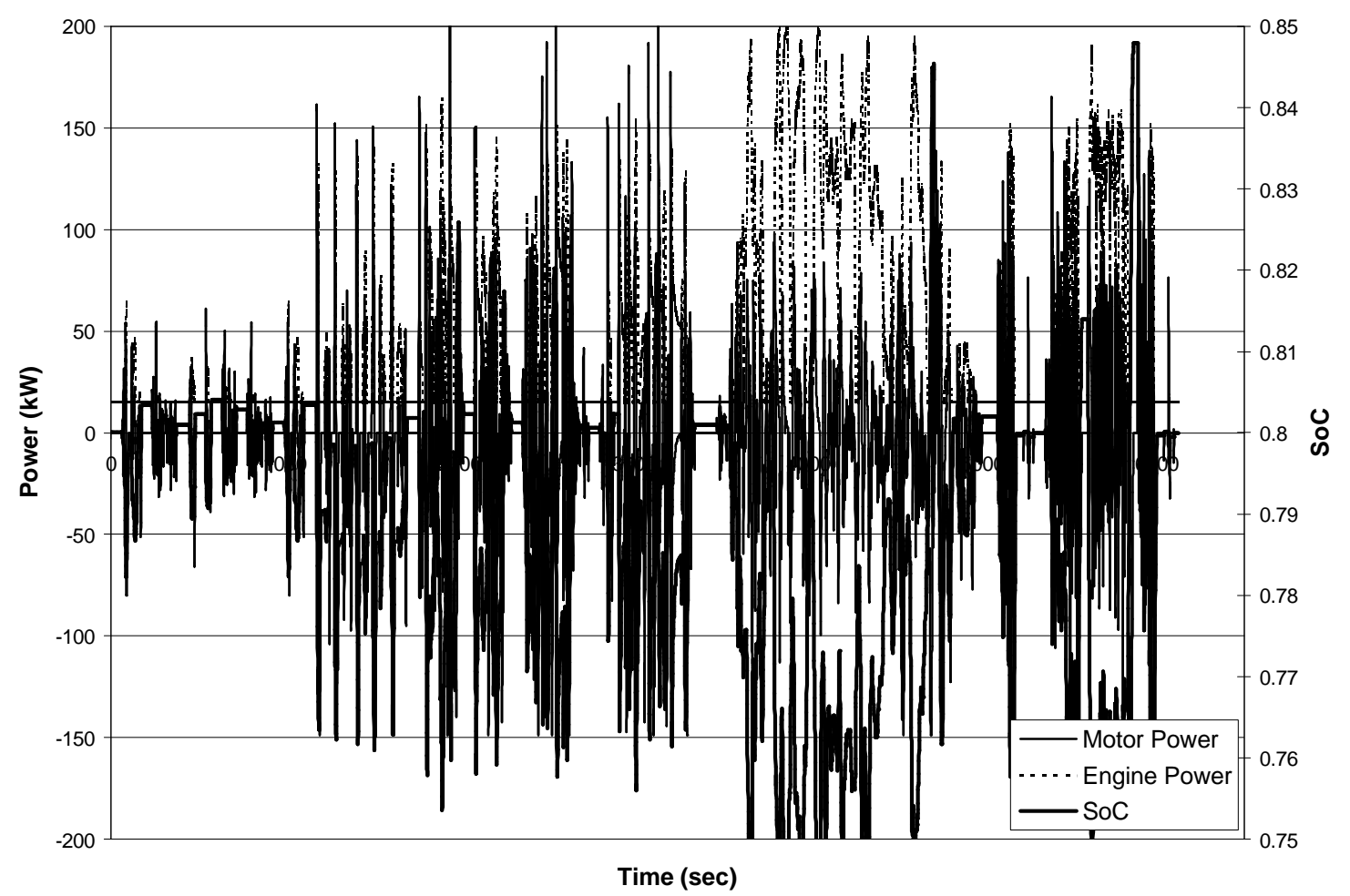

Figure A.72 Class 8 Parallel HEV on Combined Cycle with auxiliary load. 